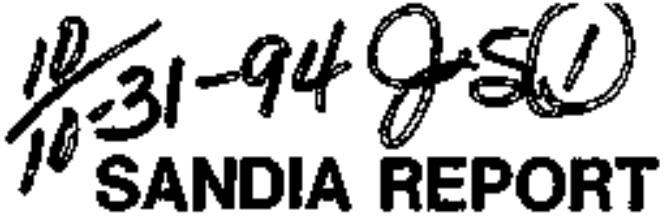

SAND94-2672 - UC-706

Unilimited Release

Printed September 1994

8

\title{
High Frequency Current Sensors Using the Faraday Effect in Optical Fibers
}

\author{
A. W. Cernosek
}

\section{Preparad by \\ 8undla Motiand Lemporatorke.

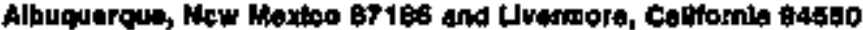 \\ For the Unibed Efates Dopertmant of Energy \\ undar Contracl DEsACO4-94ALES000}

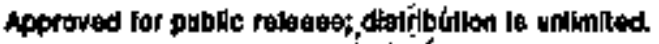

$$
-8,4
$$<smiles>[Al][V]</smiles>
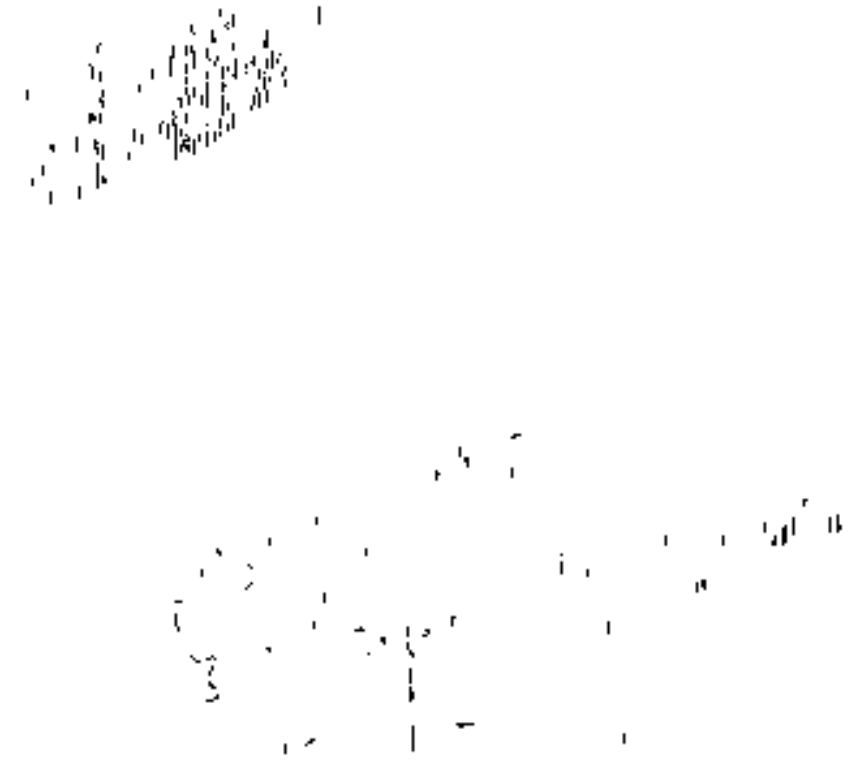

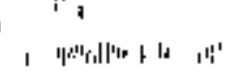

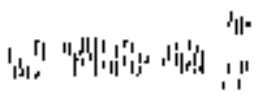

$\because-=--\overline{-}$

$-$

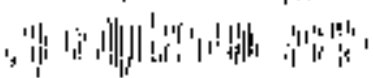


Insued by Sendia Netiongl Lahoratories, operated for the Unted States Department of Enerey by Sandia Corperation.

NOTICE: This report was prepared an account of work gponeored by an apency of the Unitad States Government. Neitlest the Unitod States Government nor any agency thereof, nor any of their employes, nor ang of their contractors, subcontractors, or their employeeg, makes any warranty, exprese or implised, or gagumes any logal linhility of responsitility for the eccuracy, completeness, ar usefuilness of any informgtion, apparatas, product, or process discloased, of represents thrt its use would not infringe privately owned rights. Referemes herein to any apediftec commercial product, process, or service by trade vame, trademark, manufacturer, or otberwises does not necessarify constitute or imply itt endorsement, recormmendation, or favoriag by the United States Goverament, any egency thereof or any of their contractors or subcontyectors. The viess and oplinions orpressed herein do not neceoarily state or reflect those of the United States Government, any asency thereof or any of their contractors.

Printed in the United States of Americe. This repart has been reproduced dírectly from the beet available copy.

A vailable to DOE and DOE contractors from

Office of Scientific and Techrical Information

PO Box 62

Osk Ridge, TN 87891

Prices available from (615) 876-8401, FTS 626-8401

Areilable to the public from

Netional Technical Information Service

US Department of Commerce

St2 5 Port Royal Rd

Springfield, VA 22161

NTIS price codes

Printed copy: All

Mierafiche copy: A01 


\section{DISCLAIMER}

Portions of this docurnent may be illegible in electronic image products. Images are produced from the best avaliable original document. 
SAND94-2572

Unlimited Release

Printed Septernber 1994

Distribution

Category UC-706

\title{
HIGH FREQUENCY CURRENT SENSORS USING THE FARADAY EFFECT IN OPTICAL FIBERS
}

\author{
R. W. Cernosek \\ Microsensor Research and Development Department \\ Sandia National Laboratories \\ Albuquerque, NM 87185
}

\begin{abstract}
This study investigates the high frequency response of Faraday effect optical fiber current sensors that are bandwidth-limited by the transit time of the light in the fiber. Mathematical models were developed for severa configurations of planar (coljocated tums) and travelling wave (helical turns) singlemode fiber sensor coils, and experimental measurements verified the model predictions. High frequency operation above $500 \mathrm{MHz}$, with good sensitivity, was demonstrated for several current sensors; this frequency region was not previously considered accessible by fiber devices.

Planar fiber coils in three configurations were investigated: circular cross section with the conductor centered coaxially; circular cross section with the conductor noncentered; and noncircular cross section with arbitrary location of the conductor. Centered, circular coil sensors have a frequency response easily characterized by light transit time. In this category, a 20 -turn, $6.4 \mathrm{~mm}$ diameter annealed fiber sensor was tested up to $1 \mathrm{GHz}$. It has a 3dB bandwidth of approximately $230 \mathrm{MHz}$ and a sensitivity-bandwidth product of approximately 0.8 MHz- $/ A$. Both noncentered coils and noncircular colls exhibit a resonant response at the higher frequencies that mimics the response of tapped, recirculating fiber delay lines. Coils with large eccentricity in the cross section feature the same sensitivity in the resonance bands as they do at low frequencies. This frequency response was successfully modeled using a point interaction between the magnetic field and the light in the fiber.

The helical travelling wave fiber coils were immersed in the dielectric of a coaxial transmission line to improve velocity phase matching between the field and light. Three liquids (propanol, methanol, and water) and air were used as transmission line dielectrics. Complete models, which must account for liquid dispersion and waveguide dispersion from the multilayer dielectric in the transmission line, were developed to describe the Faraday response of the travelling wave sensors. Large enhancements in current sensor bandwidth are possible without a loss in system sensitivity. A maximum bandwidth of approximately $300 \mathrm{MHz}$ was achieved in a water-dielectric sensor cell containing a 14-turn coil with a $3 \mathrm{~cm}$ diameter and a $3 \mathrm{~cm}$ helical pitch. This sensor has a sensitivity-bandwidth product of approximately $1.08 \mathrm{MHz}^{\circ} / \mathrm{A}$.

Other travelling wave current sensors with potentially greater Faraday sensitivity, wider bandwidth and smaljer size are investigated using the theoretical models developed for the singlemode fiber coils.
\end{abstract}

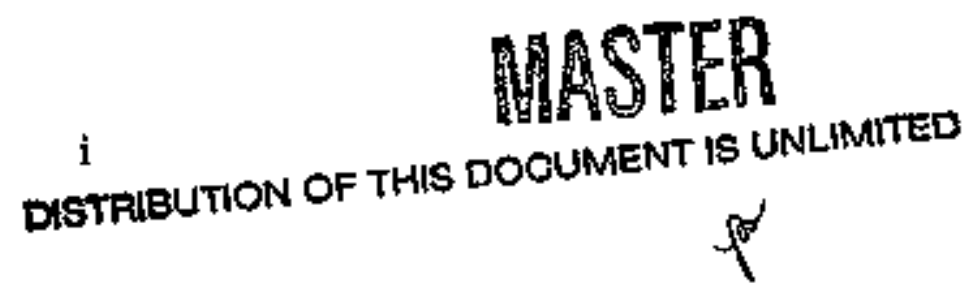




\section{ACKNOWLEDGEMENTS}

The author gratefully acknowledges the support and assistance of the following people and organjzations:

Dr. Michael A. Butter for his technical support on many theoretical and experimental problerns;

Kent B. Pfeifer for his technical assistance and laboratory support;

The Project Machining and Ceramic Processing Organizations for their fabrication of devices and fixlures;

The Electromagnetic Test and Analysis Organization for their assistance in device design and characterization;

Dr. Kenneth C. Jungling of the University of New Mexico for his technical direction: and.

Drs. Gordon W. Day and Allen J. Rose of the National lnstitute of Standards and Technology in Boulder, Colorado for their technical advice on optical fiber current sensors and their support in the fabrication and testing of the annealed fiber sensor 


\section{TABLE OF CONTENTS}

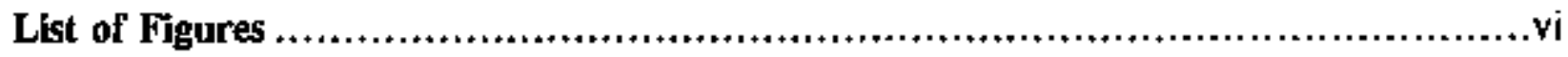

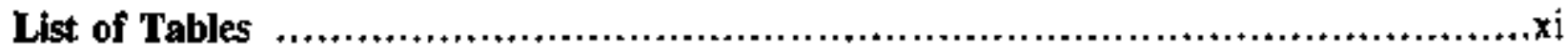

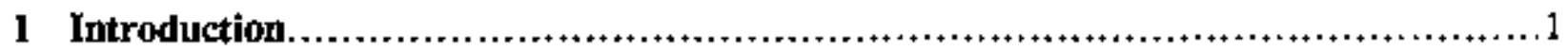

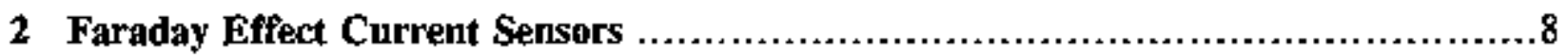

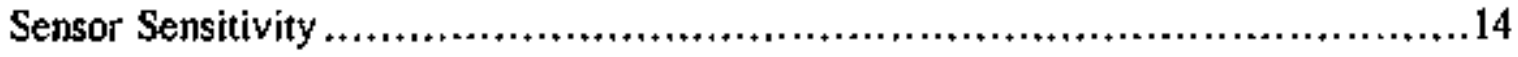

Sensor Bandwidth ................................................................19

3 Transit Time Frequency Response Theory...........................................25

4 Experimental Apparatus and Procedure.............................................31

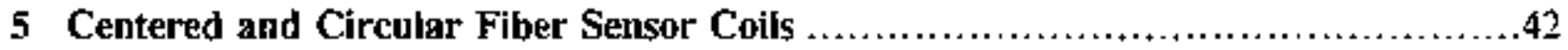

Centered Coil Model ..............................................................

Measured Response Functions ...............................................46

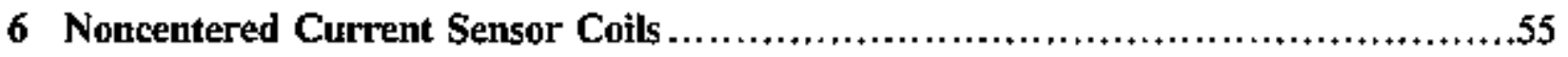

Noncentered Coil Model.......................................................55

Model Predictions.......................................................................

Noncentered Coil Measurements ................................................ 72

Conductor Outside the Coil ..........................................................

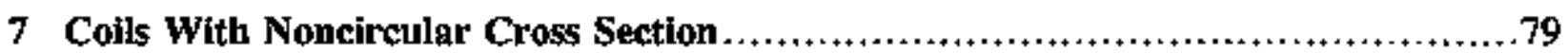

Delta Function Response Model ...................................................... 79

The Complete Response Model..................................................88

Demonstration of the Model ..........................................................91

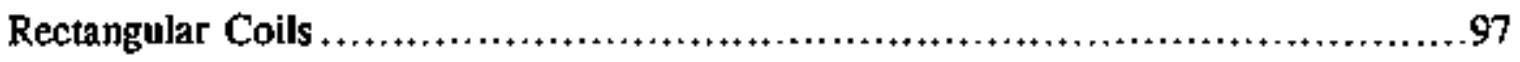

8 Travelling Wave Fiber Current Sensors; Basic Concepts and Theory .................104

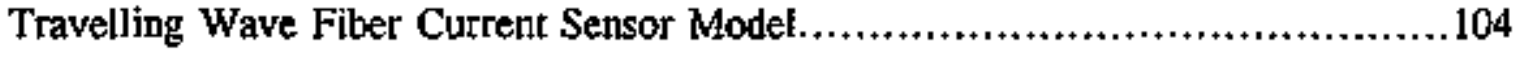

The Bandwidth Enhancement Factor .............................................108 
9 Travelling Wave Transmission Celis.

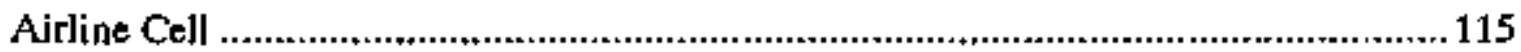

Liquid Cells...................................................................................................117

10 Extended Models for Liquid Cell Travelling Wave Sensors ......................................125

Effective Relative Permittivity ...........................................................................127

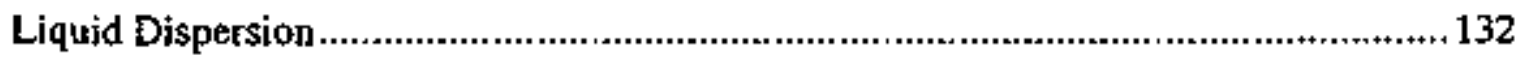

The Liquid Coaxial Waveguide ...........................................................................137

The Complete Sensor Model .................................................................................145

11 Travelling Wave Sensor Measurements.................................................................154

Airline Measurements ....................................................................................... 155

Liquid Cell Mensurements..............................................................................157

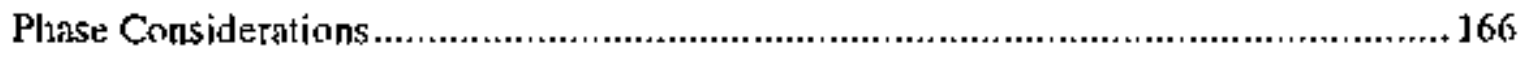

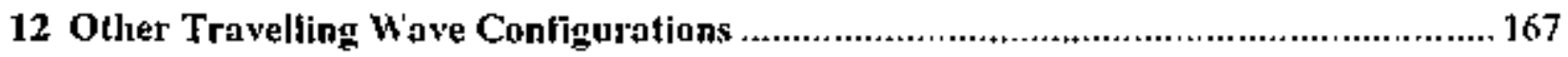

Ceramic Dielectric Travelling Wave Sensor ......................................................... 168

Combination Fiber Sensors................................................................................. 174

Non-Fiber Configurations..............................................................................177

I. Helical Waveguide Sensor .................................................................178

2. Bulk Optic Sensor.................................................................... 179)

3. Planar Waveguide Sensor .................................................................... 181

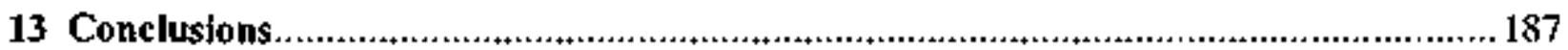

APPENDIX A: Expanded Coaxial Transmission Cels................................................ 192

Coaxial Line Properties................................................................................. 192

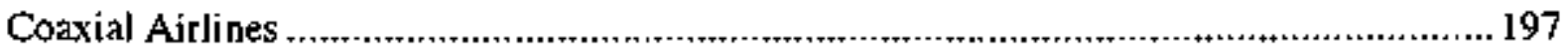

APPENDIX B: Exact Trestment of Fiber Coil Leads ................................................202

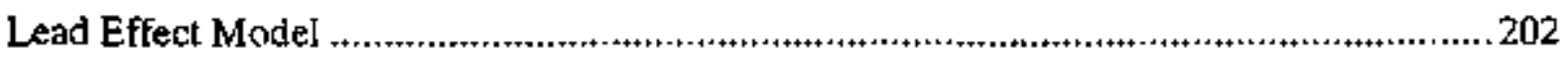

Model Results and Comparisons .....................................................................207

APPENDIX C: Derivation of Effective Relative Permittivity.......................................212

APPENDIX D: Waveguide Modes for Azimuthally Symmetric Fields in a Multilayer Coaxial Transmission LIne wlth Complex Relative Permittivity .. 


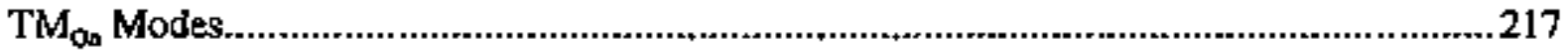

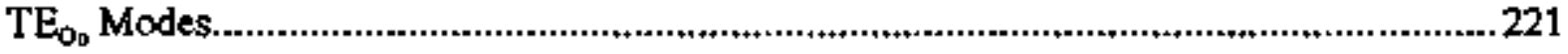

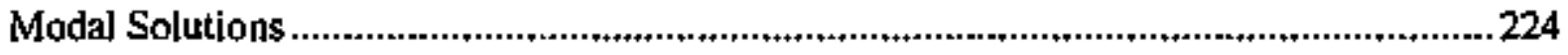

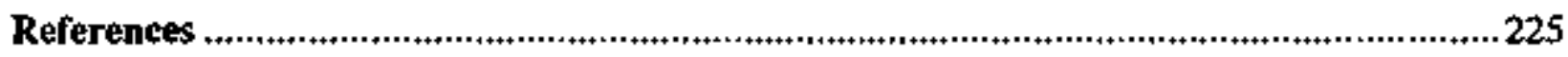

Glossary of Special Symbols and Terms .......................................................................2234 


\section{LIST OF FIGURES}

Figure $\quad$ Page

2-1. A simple optical fiber system for Faraday effect cument sensing. ................................. 10

2-2. The relative intensity response versus current for a polarimetric fiber

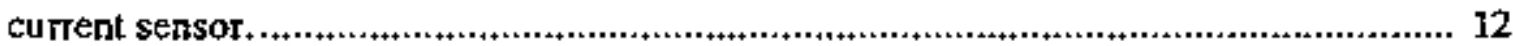

2-3. The optical output in Fig. 2-1 is split using a beam-spjitting polarizer

of a Wollaston prism for dual polarization detection.

2-4. A fiber Sagnac interferometer configured as a Faraday effect curent sensor

2-5. The noise equivalent curtent per root unit bandwidth for a single tum fitber current sensor plotted as a funclion of the average detector output current

2-6. Reiative changes in the Faraday effect versus frequency for two Manganese concentrations in $\mathrm{Cd}_{1-\mathrm{x}} \mathrm{Mn}_{\mathrm{x}} \mathrm{T}_{\mathrm{f}}[38]$.

2-7. The freguency response of the Faraday rotation for two compositions of YIG crystals

3-1. Angles formed between the lighl propagating in the fiber coil and the magnetic field associated with the conducled current.

4-1. Arrangement used for measuring the frequency response of the Faraday effect in optical fiber current sensor coils.

4-2. A photograph of the optical components on the bench top used in the measurement of tiber current sensor response.

4.3. The RF drive instrumentation used in characlerizing the response of ther current sensor cails.

4.4. Silicon PIN photodetector system built inta a shielded enciosure.

4.5. The frequency response of the silicon PIN photodiode with $42 \mathrm{~dB}$ of serial, small-signal RF amplification.

4-6 A cross sectional view of an expanded coaxial airline with an optical fiber coil mounted in the working volume.

4-7. Photographs of the 1+GHz expanded coaxial test cell.

4-8. The SWR and the impedance magnitude for the 1-GHzz coaxial test cell with an optical fiber coil inside. 
5-1. The relative magnitude and phase of the frequency dependent Faraday rotation for an optical fiber current sensor systen with a centered, circular coil.

5-2. Measured and theoretical Faraday rotation relative response magnitudes versus frequency for a 10 turn, $6 \mathrm{~cm}$ ditameter current sensor coil.

5-3. The measured Faraday rotation relative magnitudie versus frequency for a 20 turn, $6.4 \mathrm{~mm}$ diameter annealed fiber current sensor coil.

5-4. The measured signal and noise responses versus frequency for the annealed fiber current sensing system. 52

6-1. A geometrical model of a fiber current sensor coil with the conductor displaced from the coil axis.

6-2. The frequency dependent Faraday rotation response magnitude of a single tum noncentered curreni sensor coit

6-3. The effects of the light entry point in the cotl on the frequency response for a single turn, noncentered, circular coil with maximum conductor displacement $\left(X_{1} / R=1\right)$.

6-4. The frequency dependent Faraday response for a tive tum circular current sensor coil with varying relative axes displacement. 66

6-5. The effects of the number of coil turns and the light entry point on the bandwidth of a noncentered sensor coil with maximum conductor displacement.

6-6. The relative Faraday response for noncentered current sensor coils versus a frequency that has been normalized by the number of tums.

6-7. The Faraday rotation phase shift for a single tuth coil with maximum conductor displacement.

6-8. Theoretical and measured frequency response magnitudes for a $10 \mathrm{tum}$. $9.6 \mathrm{~cm}$ diameter fiber current sensor coil.

6-9. The theoretical Faraday rotation relative magnitude at the high frequencies for current sensor coils placed outside the conductor $(X 1 / \mathrm{R} \geq 1)$. 77

7-1. The "delta function" response fiber current sensor model. 80

7.2. The Faraday rotation relative magnitude versus nomalized frequency for delta function current sensor coils with two and tive turns. 85 
7-3. The relative magnitude of the Faraday rotation from Fig. 7-2 reploted

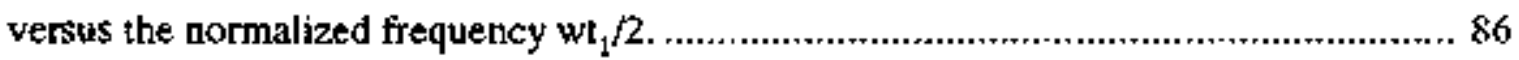

7-4. Two fiber coil configurations used to demonstrate the delta function response model.

7-5. The measured and predicted Faraday response at low frequencies (a) and higher frequencies (b) for a 10 curn test coil with short tight loops around the conductor. 93

7-6. The measured and predicted Faraday response at low (a) and higher (b) frequencies for a test coil with short straight segments passing near the conductor.

747. The geornetrical laynu for the rectangalar coil model. 98

7-8. Model predictions tor a Faraday effect fiber current sensor coil witl al varying rectangular cross section.

8-1. A travelling wave fiber current sensor constructed of a helical singlemede fiber immersed in the dielectric of a coaxial transmission line.

8-2. A typicat Faraday response curve for a travelling wave fiber current sensor.

8-3. The magnitude of the bandwidth enhancement factor as a function of the Iravelling wave coil diameter-lo-pitch ratio.

8-4. A plot of the relative permittivity of the dielectric medium required for maximum Faraday response bandwidth versus the sensing fiber diamelerto-pitch ratio.

9-1. A benchtop photograph of the experimental hardware used during testing of the travelling wave fiber cursent sensors.

9-2. Photograph of the disassembled travelling wave airline cell.

9-3. The measured SWR and impedance magnitude of the iravelling wave aitline cell with the optical fiber sensor inside.

9-4. Photograph of the liquid travelling wave transmission cell. Assembled 120

9-5. Photographs of the liquid travelling wave transmission cell. (a) End view showing inside, and (b) fully disassembled.

9-6. The SWR for the propanol-filled transmission cell for frequencies up to $500 \mathrm{MHz}$ 
9-7. The SWR for the water-filied iransmission cell for frequencies up to

$1 \mathrm{GHz}$

10-1. The static, or low frequency, effective relative permittivity versus center conductor diameter for the three liquid travelling wave tesi cells.

10-2. The real and imaginary components of the relative permittivity for a dispersive medium.

10-3. The transmission magnitude and phase angle for the propanol-filled liquid test cell used as a travelling wave sensor.

10-4. The effective relative permittivity versus frequency for the azimuthally symmeltc $\mathrm{TM}_{0_{\mathrm{n}}}$ and $\mathrm{TE}_{\mathrm{om}_{\mathrm{m}}}$ modes in a multilayer dielectric coaxial waveguide containing water. 140

10-5. The complex relative permittivity versus frequency for the $\mathrm{TM}_{01}$ mode in a propanol-filled travelling wave transmission cell.

10-6. The complex relative permittivily versus frequency for the $\mathrm{TM}_{0 \mathrm{~d}}$ mode in a methanol-filled travelling wave transmission cel!

10-7. The complex relative pemitlivity versus frequency for the $\mathrm{TM}_{01}$ mode in a water-filled travelling wave transmission cell.

10-8. The computed wave impedance magnitude for the $\mathrm{TM}_{01}$ mode in liquidfilled travelling wave cells for each of the three test liquids.

10-9. Model predictions of the relative magnitude of the Faraday rotation for a travelling wave fiber current sensor with methanol as the dielectric.

10-10. Model predictions of the Faraday cotation phase for a methanol-filled travelling wave curtent sensor.

10-11. The bandwidth enhancement tactor as a function of the diameter-to-pitch ratio for travelling wave current sensors using a methanol dielectric.

11-1. The relative magnitude of the Faraday rotation for a coaxial airline travelling wave fiber current sensor.

11-2. The experimental and theoretical Faraday response for a liquid travelting wave fiber curtent sensor containing a methanol dielectric

11-3. A comparison of the relative Faraday rotation for each of the three lest liquids used with the same helical coil. 
11-4. The relative magnitude of the Faraday rotation for iravelling wave current sensor with a 20-tum helical coil in a methanol-filled transmission cell.

11-5. The measured and modeled responses for a travelling wave fiber curient sensor using a coil with $\mathrm{N}=14, \mathrm{~d}=3 \mathrm{~cm}$ and $\mathrm{d} / \mathrm{x} 0=1.0$ inside a cell containing water. 162

11-6. The measured relative magnitude of the Faraday rotation for the largest bandwidh travelling wave sensors using each of the three test liquids.

12-1. A composite drawing of a travelling wave sensor transmission cell with a ceramic dielectric.

12-2. Photographs of the ceramic dieleclnic travelling wave transmission cell, 171

12-3. The measured impedance nagnitude of the ceramic dielectric transmission cell for frequencies belen. $S \mathrm{GHz}$.

12-4. The predicted frequency dependent magnitude and phase of the Faraday rotation for a travelling wave current sensor using a ceramic dielectric and an annealed helical fiber coil.

12-5. The relative magnitude of the Faraday rotation for a two-lum travelling wave fiber sensor with the current conductor fully displaced in the coil cross section

12-6. A wideband bulk optic current sensor with a helical wire coil used to generate an axial magnetic field inside a cylindrical piece of Faraday rotator material 180

12-7. A planar waveguide travelling wave optical cusrent sensor. 182

12-8. The predicted relative magnitude of the Faraday rotation for the planar waveguide travelling wave current sensor. 184

A-1. The geometry of a coaxial transmission line. 193

B-1. A geometrical model of the fiber leads on a current sensor coil 203

B-2. The predicted frequency response for a single tum Faraday effect fiber current sensor with two different lead configarations. 208

B-3. The effect of lead length on the relative response magnitude of a single tum fiber current sersom...............................................................................................210

C-1. Cross section of the multilayer dielectric conxial line...............................................212 


\section{LIST OF TABLES}

Table

2-1. Verdet Constants for Several Materials use in Faradiay Effect Sensors.

5-1. Summary of High Frequency Response Measurements on Centered,

Circular Optical Fiber Current Sensor Coils .

6-1. Changes in the $3 \mathrm{~dB}$ Bandwidth for Noncentered Fiber Current Sensor

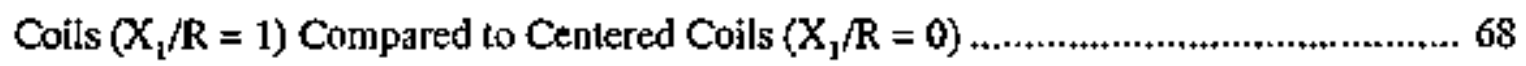

9-1. Relative Permittivity of the Test Liquids at Selecled Temperatures................................118

9.2. Relative Permitivity of Water .............................................................................119

A-1. Expanded Conxial Airline Cetls .........................................................................200 


\section{CHAPTER I}

\section{INTRODUCIION}

The use of optical methods to measure environmental parameters and physical quantities is becoming highly cornmonplace in today's technology. Optical sensors, or more commonly photonic or fiber optic sensors, can perform very sensitive measurements often with the inherent speed afforied by the optical processes and with the added advantage of making remote and nonperturbing interrogations. In recent years, photonic sensing has found use in applications for characterizing electromagnetic (EM) quanlities [1-3]. Here, the reduced interference of the optical or dielectric media serves a dual purpose: the presence of the instrumentation, or sensor, does not perturb the environment being measured like conductive materials often can; and the measurement itself is EMI (electromagnetic interference) -proofed, the environment does not perturb the measurement. This property has been the main reason that optical technoiogy is being developed for detection of EM parameters, since in general, photonic sensors have yet to attain the high frequency sensitivities or the sensitivity-bandwidth products achieved by the conventional techniques. This is particularly true for electrical current sensors. Wideband operation that extends well into the microwave region (beyond 500 $\mathrm{MHz}$ ) is desired while maintaining reasonable current detection sensitivity.

Conventional wideband electrical probes use an inductive loop or inductive transformer to sense current. These devices are quite sensitive, capable of measuring less than $1 \mathrm{~mA}$ near their upper freguency limit of $\sim 1 \mathrm{GHz}$ [4]. However, extending probe frequency response mach beyond $1 \mathrm{GHz}$ is unlikely due to the inductance associated with the transouction fechnique. Other conventional methods exist that measure steady state currents at RF and microwave frequencies (tens of $\mathrm{GHz}$ ); most have applications for determining weapon component susceptibility and ordnance safety. These probes sense the themal tise produced by Joule heating when current passes through a resistance point 
in the conduction path. Most common are electrical thin film thermocouples and thermistors used to measure currents in electroexplosive devices (EEDs) [5]. Fiber optic thermometric techniques have been developed that replace the themocoople/thermistor to deternine currents and powers in EEDs or substitvie resistors [6]. These hybrid optical-electrical sensors still incur some signal perturbations, and since they record only the magnitude of the RF currents they are incapable of measuring transients faster than the thermal response lime.

Two competing optical techniques can be used to measure magnetic field or curtent waveforms: magnetostriction and Faraday rotation. Each has its advantages [3], and both have been adapted to optical fiber current sensing. In magnetostrictive sensors [7] an externai magnetic field produces a deformation or strain in a magnetostrictive material that is in intimate contact with a singlenode fiber. The strain is transferred to the fiber creating perturbations in geonetry and refractive index. Deviations in the oplical path length are sensed interferometrically. This technique is quite sensitive; magnetic frelds as small as $0.07 \mathrm{pT} / \sqrt{\mathrm{Hz}}$ have been detected [8]. However, bandwidihs are generally limited to a few tens of kilohertz because of the mechanical movement reguired in the magnetostrictive material. Sensors with frequency response beyond 1 MHz have been constructed by mixing the signal with a field from an RF oscillator [9]; the detection band is shifted to a higher center frequency but the detection bandwidth remains unchanged.

The Faraday effect, on the other hand, is an inherently fast optical process in most materials. In the sensing medium, the magnetic freld produces a rotation in the plane of polarization of the propagating light. Field and current sensors which exploit this fast process have been reported [10-14]. Measurement at frequencies above $1 \mathrm{MHz}$ are easily accomplished, afthough response sensitivities do not approach those of the magnetostrictive sensors. The strength of the Faraday interaction is relatively weak in most materials. Common silica optical fibers exhibit particularly small rotations per unit 
length. As a result, fiber sensors have mainly been used to measure kiloampere or megampere currents [15] most notably produced in the power generation [16-17] and fusion research $[11,18-20]$ industries. (In these applications high electrical isolation is definitely a benefit.)

Much effort in recent years has concentrated on improving the sensitivity of Faraday effect fiber current sensors. Sensitivity is improved by increasing the interaclion length between the field and the light or by using materials that produce a larger Faraday rotation per unit length (possess a larger Verdet constant). Longer fiber lengths, however, often lead to large, bulky sensors while limiting bandwidth due to light transil time. Recent investigations into fiber annealing [21,22], circularly-birefringent [23] and elliptically-birefringent [24] singlemode fibers, higher Verdet constant fibers [25-27], and other special fiber processes $[28,29]$ have allowed for the fabrication of small, sensilive multiturn coils and provided the direction needed to achieve subampere current detection using optical fiber systems.

Other material studies have identified the ferromngnetic (or ferrimagnetic) crystais such as the iron garnets $[12,13,30,31]$ and paramagnetic crystals such as ComnTe [10,32] as possessing excellent Faraday sensitivity for field sensing applications. Rotation sensitivity in these crystals exceed that in silica by more than three orders of magnitude. Also, one current sensor configuration has recently been developed using the ferromagnetics [30]. The huge increase in detection sensitivity, however, often means that frequency response is reduced due to the reguired reorientation times of the magnetic ions or domains in the optical material.

The tradeoff between sensittvity and bandwidth is apparent in all classes of magneto-optic materials and processes and, subsegnently, in any field or current sensor that utilizes them. Diamagnetic glasses and crystals (silica fibers belong to this group) have small Faraday sensitivities but possess extremely wide material bandwidths. For this reason, optical fiber current sensors are always limited in high frequency response by 
the transit lime of the light through the fiber. Thus, tradeoffs between sensilivity and bandwidth require compromising on fiber length. For most applications the required current detection sensitivity determines the fiber and coil parameters and response bandwidth is estimated from the fiber length. This practice is widely accepted, and little attention is given to bandwidth considerations during sensor design unless large currents are being detected. However, many current monitoring applications require wideband detection of milliampere-level signals. Faraday effect sensors, and particularly optical fiter sensors, have the potential to fill these needs should their high frequency response be better understood.

A formal characterizalion of the frequency response of fiber current sensors, including expressions for $3 \mathrm{~dB}$ bandwidth and the sensitjvity-bandwidth relationship, has recently been presented [33]. The theoretical discussions in Ref. 33 are mosily limited to the simplest of fiber sensor coils, those that are circular and concentric with the current conduclor. In addilion, experimental measurements are performed on a single coil at frequencies up to $5 \mathrm{MHz}$. which is limited to just above the sensor $3 \mathrm{~dB}$ bandwidth.

In this sludy a complete picture is given for the high frequency response of Faraday effect fiber current sensors. Theoretical models are generated and response functions are meastred for many different fiber coil configurations. These include sensors with planar, circular coils centered on the axis of the current conductor; ciscular sensing coils with the axes displaced from that of the conductor, noncircular or elongated fiber coils with concentric and displaced axes; and helical coils aligned coaxially with the conductor. Varying coil diameters and the number of coil turns further extends each category. Many other configurations (some of them non-physical) are explored theoretically to provide further insight into current sensor models. Measurements are made for frequencies between $1 \mathrm{MHz}$ and $1 \mathrm{GHz}$. For many sensor coils this represents frequencies well above the $3 \mathrm{~dB}$ bandwidth and the response function cutoff frequency. Fiber current sensing in this frequency range has not been demonstrated or reported 
previously.

Planar fiber coil sensors - those with collocated or closely assembled turns have well-defined bandwidths as detemined primarily by light transit time. Manipulations of the coil cross section or off-center placement of the conductor axis within the coil introduces response resonances at higher frequencies. These can be exploited for narrowband current sensing; demonstration of the technique is performed at frequencies above $500 \mathrm{MHz}$. Within the high freguency bands, relative increases in detection sensitivity occur, while the bandwidth remains as described by the lower frequency response.

The fundamental bandwidth limitation dictated by the light transit time in the liber current sensor can be overcome by using travelling wave technigues. Improved phase matching between the propagating magnetic field and the light in the fiber is accomplished by spreading the coil twins in a helix then immersing the coil in the dielectric of a coaxial uansmission line. This can be accornplished without a loss in measurement sensitivity, thereby increasing the sensitivity-bandwidth product of the Faraday effect current sensor. Demonstrations of the technique use liguids as the dielectric medium in the slow-wave transmission line structure. Measured bandwidths of $300 \mathrm{MHz}$ with bandwidth enthancenents of greater than four (compared to planar coils) are reported. Because of the orientation of the magnetic field relative to the light in the 1iber, phase matching in a Faraday effect travelling wave current sensor is much more difficult to achieve than in electro-optic travelling wave field sensors. Perfect phase matching is virtually impossible to attain, although an optimum set of sensor travelling wave parameters exists such that large bandwidth enhancements are achievable.

Following this introduction, Chapter 2 gives an overview of the Faraday effect in bulk optics and optical fibers with specific applications to current sensing. Limitations on measurement sensitivity and bandwidth are discussed in relation to material properties, fiber birefringences, and common system noise sources. Chapter 3 presents 
the general theoretical description of the frequency dependence of the Faraday rotalion in fiber current sensors when light transit time through the fiber is the constraining factor. These mathematical developments are then continued in the later chapters as specific model responses are generated for particular fiber sensor coil configurations. After Chapter 3 , this document branches in two fondamental directions. Chapters 4 through 7 cover fiber current sensors that utilize planar coils, while Chapters 8 through 12 discuss travelling wave fiber sensors with belically shaped coils.

The details of experimental hardware and procedures are presented in Chapter 4. Included among the hardware is the expanded coaxial transmission cells that contain the fiber coils and produce the magnetic fielos [34]. Additional discussion about these cells is given in Appendix A. Many of the apparatus and procedural details related in Chapter 4 and Appendix A also apply to the travelling wave sensors. Starting with Chapter S. mathematical models and expcrimental measurements of the frequency dependent Faraday response are presented for specific categories of fiber coil configurations. Chapter 5 covers circular coil sensors with the current conductor centered in the coil cross section. Several coils with varying geometry are characterized, highlighted by tescing of a small diameter $(6.4 \mathrm{~mm})$ annealed coil sensor. Noncentered, circular coil sensors are discossed in Chapter 6 . This discussion includes theoretical response predictions for a sensor systen with the conductor totaly outside the coil cross section. Chapter 7 presents fiber coils with noncircular cross sections. "Delta function" or point interaction models are developed from a highly eccentric coil geometry. Rectangular coils are useo to describe the Faraday response in generic noncircular fiber sensors. Important in the accurate determination of current senşor frequency response is the inclusion of effects due to the fiber coil leads. Appendix B gives an exact mathematical treatment of these lead effects for a planar coil geometry.

Chapter 8 begins the study of the travelling wave fiber current sensors with a discussion of the basic sensing concepls. Bandwidth enhancernent is a key element in the 
use of the travelling wave configurations and performance criteria are established in this chapter. The experimental travelling wave transmission cells are presented in Chapter 9. Cells contain either air as a dielectric or one of three liquid dielectrics: 1-propanol, methanol, or water. The peculiarities associated with precise sensor response modeling for the liquid travelling wave structures are detailed in Chapter 10. Complete models are developed and include effects created by liguid dispersion, waveguide dispersion and multimoding in the coaxial transmission cells, and relative permittivity perturbations due to fiber coil supports. Mathematical supplements to the models are provided by Appendices $\mathrm{C}$ and $\mathrm{D}$ which derive the effective relative permittivity in the coaxial lines and the waveguide modes in a multilayer dielectric coaxial line with complex relative permitlivity. Chapter 11 presents the experimental measurements using the fiber Iravelling wave sensors and compares the Faraday response data to model prediclions.

Further enhancements to the bandwidth and high frequency response of Faraday effecl current sensor's are discussed in Chapter 12 for several other travelling wave configurations. These include solid dielectric coaxial transmission line stroctores, conbination travelling wave/noncentered (or noncircular) fiber coil sensors, helical waveguide sensors using higher sensitivity materials, bulk-optic helical-condnctor travelling wave sensors, and a serpentine, planar waveguide in an RF stripline. Finally, Chapter 13 summarizes the high frequency response model predictions and experimental measurements for all planar and travelling wave fiber current sensors. 


\section{CHAPTER 2}

\section{FARADAY EFFECT CURRENT SENSORS}

The Faraday effect is an induced circular birefringence created by a magnetic fiefd interacting with an optical medium [35]. The resulting rotation of the plane of polarization, $\ominus$, of light propagating in a medium of length $L$ is described (in MKS units) by

$$
\theta=V \int_{0}^{L} B \cdot d l .
$$

Only that component of the magnetic flux densily, B, which lies in the direction of the incremental lengll, dl, of the light propagation contributes to the effect. The proportionality factor, $V$, is the Verdet constant and determines the strength of the interaction created by the field. The Verdet constant is a material parameter that is also dependent upon the temperature [36], the wavelength of the propagating light and the modulation frequency of the magnetic field $[37,38]$. Verdet constants for some common materials used in sensors are given in Table 2-1 $\{39,40]$.

Current sensors using the Faraday effect can be quite simple. Ampere's law states that for a static magnetic field $\mathrm{H}$, the current, $\mathrm{i}$, is given by

$$
\mathbf{i}=\oint \mathbf{H} \cdot \mathbf{d}:
$$

Thrs, allowing the interrogating light beam to propagate in a loop around the current conductor produces a rotation that is independent of the beam path. Optical fibers wound in a coil represent an ideal method for directing light around a current carrier. Multiple 


\section{Table 2-1.}

Verdet Constants for Several Materials Useo in Faraday Effect Sensors.

Parameters were measured at a wavelength of $633 \mathrm{~nm}$ and at $25^{\circ} \mathrm{C}$. (Refs. 39,40 ),

$\begin{array}{ccc}\text { Material } & \text { Type } & \mathrm{V}(\mathrm{rad} / \mathrm{T} / \mathrm{m}) \\ \mathrm{Cd}_{1-x} \mathrm{Mn}_{x} \mathrm{Te} & \text { Paramagnetic Crystal } & -2000 \\ \mathrm{ZnSe} & \text { Diamagnetic CrystaI } & 118 \\ \mathrm{Bi}_{4} \mathrm{Ge}_{3} \mathrm{O}_{12} & \text { Diamagnetic Crystal } & 28.8 \\ \mathrm{FR}-5 & \text { Paramagnetic Glass } & -71 \\ \mathrm{FR}-4 & \text { Paramagnetic Glass } & -30.5 \\ \mathrm{SiO}_{2} & \text { Diamagnetic Glass } & 3.6 \\ \mathrm{SF}-57 & \text { Diamagnetic Glass } & 20\end{array}$

torns of the fiber have the effect of amplifying the resulting Faraday rotation. Such a sensor bas a rotation described simply by

$$
\Theta_{0}=\mu_{f} \vee N i,
$$

where $\mu_{i}$ is the permeability of the interaction medium, usually taken to be that of free space since materials are typically nonmagnetic dielectrics, and $\mathrm{N}$ is the number of loops of optical fiber. Sensitivity to currents can be enhanced even further by using several turns of the conductor through the optical fiber coil to increase the magnetic field. The zero subscript used in Eq. (2-3) denotes the dc or low frequency rotation produced by a static or slowly-varying magnetic field. If the amplitude of the field changes 
significantly in the time it takes the light to traverse the closed path, then Ampere's law, Eq. (2-2), is no longer valid.

A typical current sensing system asing an optical fiber [41] is shown in Fig. 2-1. The light output from a laser source is passed through a polarizer injecting linearly polarized light into the fiber. Light exiting the fiber is analyzed with a second polarizer converting induced rotations to transmitted intensity changes that can be detected by the photodiode receiver. The sensing fiber must be singlemode in order to preserve the instantaneous polarization state of the light. The measured light intensity, I, for the system in Fig. 2-1 when the polarizers are aligned is given by

$$
I=I_{0} \cos ^{2} \Theta_{0}
$$

where $l_{0}$ is the maximum transmitted light intensity and $\Theta_{0}$ is taken from Eq. (2-3). For a system using crossed polarizers, the transmitted intensity is

$$
I=I_{0} \sin ^{2} \Theta_{0} \text {. }
$$

For this case, the relative inlensity is zero with no current. The intensity reaches a

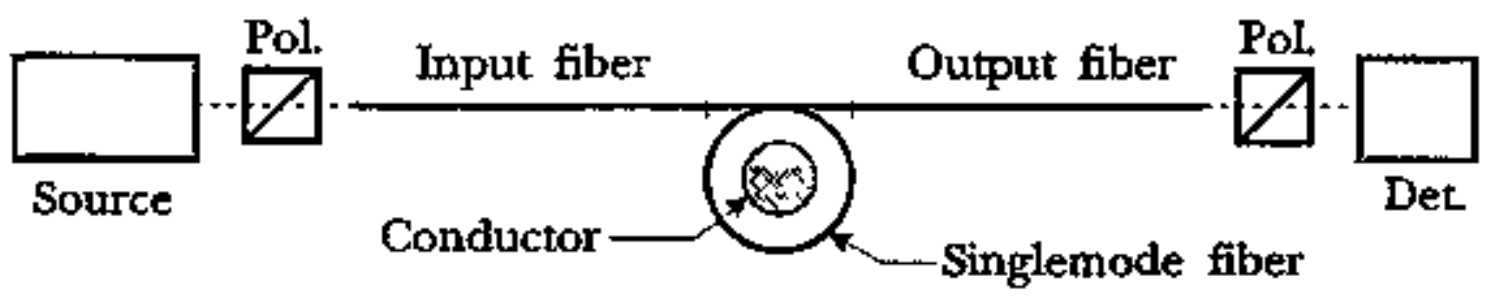

Fig. 2-1. A simple oplical fiber system for Faraday effect current sensing. This polarimetric configuration uses singlemode fiber between input and output polarizers. 
maximum when a current is present that produces a rotation of $\Theta_{0}=\pi / 2$, or odd miltiples of $\pi / 2$. It is obvious from Eqs. (2-4) and (2-5) that a measured intensity could represent any number of currents. This has an advantage in current sensing since saturation cannot occur as in magnetic core cursent probes. However, difficulties can be encountered when determining large currents where several of these intensity "fringes" are observed. Usually in this case, two polarization states $45^{\circ}$ apart are monitored simnltaneously at the fiber outpus [42]. A ratio of the two detected signals gives an unambiguous determination of the current. The relative intensity response of the fiber sensor in Fig. 21 is plotted in Fig. 2-2 as a function of measored cursent. The effect of several polarizer configurations are shown.

Srnall currents are measured with the greatest sensitivity in a system using input and output polarizess oriented at $\pi / 4\left(45^{\circ}\right)$ with respect to one another. The transmitted relative intensity at zero current (see Fig. 2-2) is biased to $1 / 2$, and bipolar current signats produce an increase or decrease in intensity about this point. Mose importantly, the intensity slope, $d / d i$, is maximum. If the currents are srnall enough so that rotations are much less than $\pi / 4$, then measured changes in intensities are linear with changes in current. From Fig. $2-2$, polarizers aligned at $+45^{\circ}$ and $-45^{\circ}$ produce equivalent intensity changes but with different signs. Sensing both of these polarization states simultaneously and then taking the difference in intensities produces a measurement with twice the sensitivity. A system incorporating this scherne is identical to that in Fig. 2-1, except the output polarizers and delectors are arranged as in Fig. 2-3. The polarizing beam splitter selects the orthogonal polarization components, one for each photodiode receiver. This system has a response with rotation, or current, of

$$
I=I_{0} \sin \left(2 \Theta_{0}\right)
$$

Using this differential detection scheme also reduces systern common mode relative 


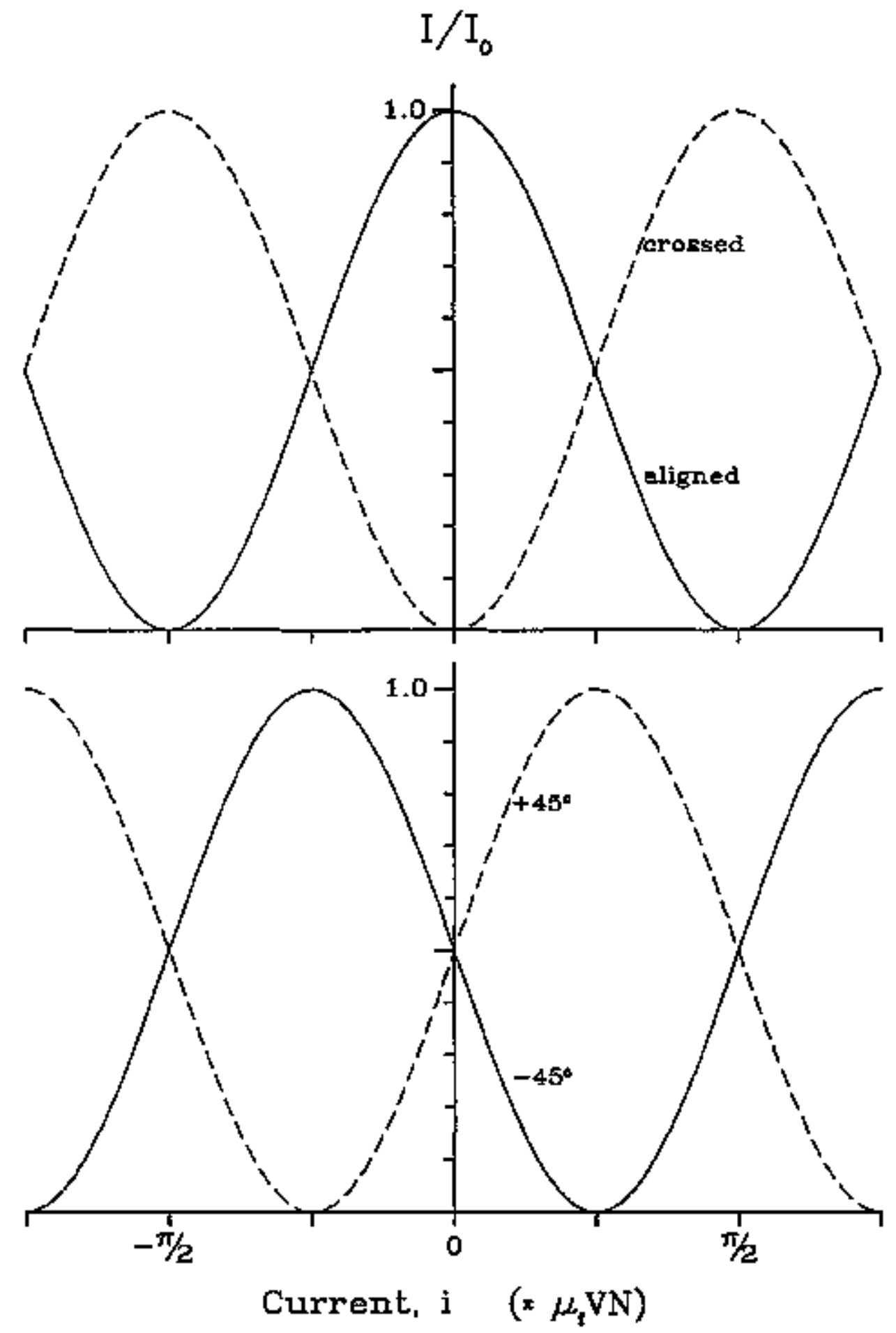

Fig. 2-2. The relative intensity response versus current for a polarimetric fiber current sensor. (Top) With polarizers aligned and crossed. (Bottom) With polatizers oriented at $\pm 45^{\circ}$ to one another. 


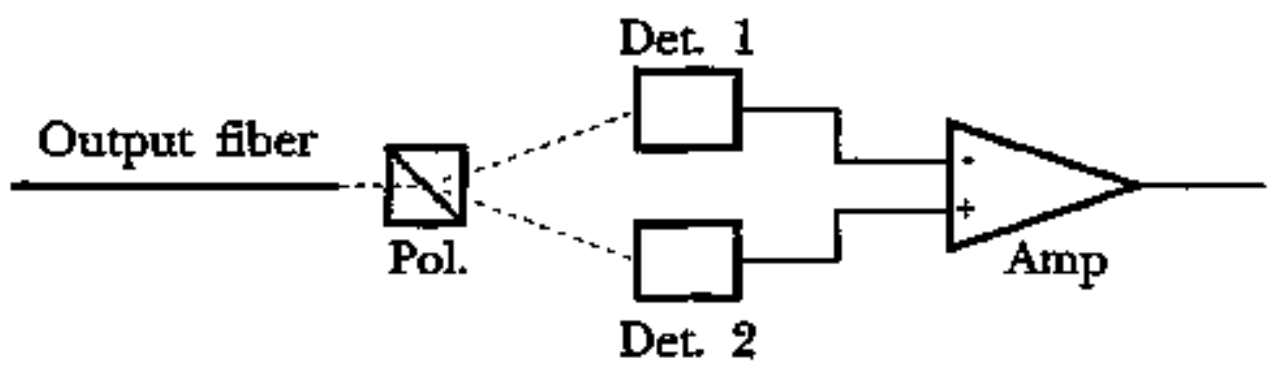

Fig. 2-3. The optical output in Fig. 2-1 is split using a beam-splitting polarizer or a Wollaston prism for dual polarization delection.

intensity noise such as canjer fluctuations in semiconductor diode lasers.

In addition to the polarimetric sensing schemes illustrated in Figs. 2-1 and 2-3, optical fiber Faraday effect current sensors can also be constructed using interferometers. In particular, the Sagnac interferometer [43], shown in Fig. 2-4, provides a very simple method for sensing curtents. The counter-propagating beams in the fiber are rotated nonrecipracally by the magnetic field resulting in a doubling of the detected response compared to the polarimetric sensor. Reciprocal effects produced by some noise sources (e.g., laser phase noise and stress-induced polarization changes from temperature drift), however, are inherently compensated for when the two signals are combined at the fiber coupler. The Sagnac interferometric sensor also has the advantage of not requiring exlernal polarizers. Its greatest drawback is that the response is likened to that of crossed polarizers in the polarimelric current sensor. The detected intensity is zero with zero slope, as shown in Fig. 2-2, when no current is present. Recently, corrent sensors that use the fiber Sagnac interferometer with a $3 \times 3$ fiber coupler have been studied [44]. With this system, it is possible to bias the zero current response to a relative intensity of $1 / 3$, with a nonzero response slope. This fiber cument sensor has many advantages for detection of small currents.

Faraday effect current sensors are not limited to optical fibers, atthough wotking 


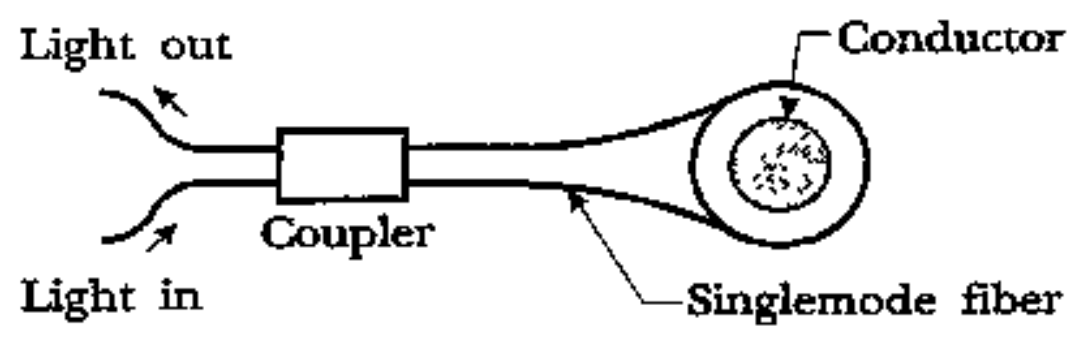

Fig. 2-4. A fiber Sagnac interferometer configured as a Faraday effect current sensor. The fiber coupler launches two counter-propagating beams of light that are recombined at the coupler output for optical delection.

with fibers is usually much easier. Several glasses have been used to constuct sensors that either slip around a custent conductor or have the conductor pass through a hole in thern $[45,46]$. Also, a Ga:YIG (fenromagnetic crystal) sensor, consisting of four separale rods that form a path around the conductor, has been developed [30]. For these bulk oplic sensors, materials are selccted that have much higher Verdet constants or much larger rolations per unit field than a silica fiber. This leads to a more sensitive current detection system. Fibers are still necessary to transport light to and from the basic sensor, ano lenses and other optical elements are required to collinate and direct the light through the bulk glass.

\section{Sensor Sensitívity}

Faraday effect optical fiber current sensors do not always have an ideal response that is only dependent on the optical rotation. Singlemode fibers axe a birefringent medium and controlling the exact state of polarization of the propagating light is difficult $[47,48]$. Stress introduced in the fiber during manufacturing or simply by winding it into a coil creates a linear birefringence, or retardance, that is coupled to the circular birefringence produced by the Faraday rotation. This effect can mask the rotation, resulting in loss of sensor sensitivity. Inherent stresses due to manufacturing can be 
reduced significantly through careful processing [49]; however, bending stresses cannot be reduced quite so easily. Fortunately, the linear birefringence induced by bending is controllable and well defined [50]. For a fiber under zero tension, the bend-induced birefringence per unit length is given as

$$
\Delta \boldsymbol{\beta}=\mathrm{K}_{\lambda} \frac{\mathrm{I}_{f}^{2}}{\mathrm{R}^{2}},
$$

where $r_{f}$ is the fiber radius, $R$ is the bend or coil radius and $K_{\lambda}$ is a parameter related to the stress-optic coefficient and dependent upon the fiber material and the wavelength of light. For a silica fiber with $\lambda=633 \mathrm{~nm}$ (HeNe laser light), $K_{\lambda}=7.7 \times 10^{7} \mathrm{deg} / \mathrm{m}$.

In a linearly birefringent medium, the effects of Faraday rotation are best expressed as an operation on the complex field anplitudes, $\mathrm{E}_{\mathrm{x}}$ and $\mathrm{E}_{\mathrm{y}}$. Using the Jones Caiculus, these amplitudes are described by [51]

$$
\begin{gathered}
\left|\begin{array}{l}
E_{x}(z) \\
E_{y}(z)
\end{array}\right|=\left|\begin{array}{cc}
A & -B \\
B & A^{*}
\end{array}\right| \begin{array}{l}
E_{x}(0) \\
E_{y}(0)
\end{array} \mid, \\
A=\cos \left(\frac{\phi^{\prime}}{2} z\right) \cdot j \frac{\Delta \beta}{\phi^{\prime}} \sin \left(\frac{\phi^{\prime}}{2} z\right), \\
B=\frac{2 F}{\phi^{\prime}} \sin \left(\frac{\phi^{\prime}}{2} z\right),
\end{gathered}
$$

and

$$
\left(\frac{\phi^{\prime}}{2}\right)^{2}=\left(\frac{\Delta \beta}{2}\right)^{2}+F^{2}
$$

Here, $\Delta \beta$ is the total retardance per unit length and $F$ is the total Faraday rotation per unit length. The field amplitudes are assumed to have a known state at $z=0$, the entrance to 
the fiber coil. The axes for linear birefringence are also assurned to remain constant throughout the fiber coil, with the fast axis in the plane of the fiber bend. From Egs. (2-8), it is seen that if $F>\Delta \beta$, the operation on the field amplitudes is a pure rotationConversely, if $\Delta \beta \gg F, B \rightarrow 0$ and a pure retardance results. Under the fatter condition, the sensing of Faraday rotation can be quenched.

The effects of linear birefringence in the fiber sensor coil can be reduced in several ways. Twisting of the optical fiber along its axis introduces a circular birefringence that acts to bias the sensor response to Faraday rotation $[47,52]$. The twisting rotation per unit length, $\Omega^{\prime}$, is given by

$$
\Omega^{\prime}=\mathrm{g}^{\prime} \xi_{\mathrm{f}},
$$

where $\xi_{r}$ is the number of twists per unit length and $g^{\prime}$ is a material dependent parameter. At $\lambda=633 \mathrm{~nm}, \mathrm{~g}^{\prime} \approx 0.08$. The rotation induced by twisting adds algebraically to the field-induced Faraday rolation to produce the quantity, F, required for Egs. (2-8). Since Iwisting has a nonsymmetric effect on bipolar signals, it is important that care be taken in sensor coit design.

In general, most commercially available optical fibers can withstand a twist rate of 50 turns per meter [15]. This is more than adequate to offset the effects of linear birefningence in large diameter coils, but in fiber coils produced of many small turns, it is not sufficient. More rotation can be achieved through the use of circularly and elliptically birefringent optical fibers. A circularly birefringent fiber is manufactured with a helical core [23]; elliptically birefringent fibers are produced from high linearly birefringent fiber by spinning the preform doring the fiber pulling process $[24,53]$. Corrent sensors constructed from these fibers have led to relatively high sensitivity measurements.

The linear birefringence in small multiturn fiber coils can essentially be 
eliminated through annealing $[21,22,54]$. The fiber is wound in the desired geometry, heated to near the glass softening point and then retumed slowly to room temperature. The annealed coil retains its predetermined configuration but has orders of magnitude less linear birefringence with only a slight increase in attenuation. Fiber sensors produced using this technigue have resulted in the highest sensitivity measurements to date, noise equivalent currents of $-100 \mu \mathrm{A} / \sqrt{\mathrm{Hz}}$ for 188 turns of silica fiber in a coil 1 $\mathrm{cm}$ in diameter [54].

Improved sensitivity is also possible through the use of higher Verdet constant optical materials. Many materials are available that have a much greater current measurement potenlial than do silica fibers (see Table 2-1). Attempts have been made to produce optical fibers from special glasses [25], such as FR-5, and from silica with high concentrations of ratc earth dopants $[26,27]$. In general, the resulting fibers have contributed litle to sensor applications because of large increases in fiber attenuation, increased linear birefringence resulting from the manufacturing process or only minor improvernents in Verdet constant for dopants in a stable glass matrix. Should production of quality higher Verdet constant fibers become prossible, one material in particular is of interest: SF-57 glass [45], with a Verdet constant 5.5 times that of silica and with a stress-optic coefficient (for reduced bend birefringence) about 100 times less.

Bulk optic sensors have been fabricated from the diamagnetic materials SF-57 glass and polycrystalline $2 n \$ e$ [46]. These devices show increases in Faraday rotation that reflect their larger Verdet constants. Most bulk materials have been used for magnetic field sensors, but configurations for current sensors have been developed $[45,46]$. Temperatore dependence of the Verdet constant and thermal expansion of the optical material set a limit on the measurement precision of the Faraday rotation. Diamagnetic materials are typically less temperature dependent than paramagnetic or ferromagnetic materials [35] which often makes them more desirable for sensor applications. A compensation mechanism for reducing the temperalure dependence in 
diamagnetic materials through use of input polarization control has also been developed [55].

For greater Faraday sensitivity, the pararnagnetic materials with even larger Verdet constants can be used. One in particular is the dilute magnetic semicondoctor, $\mathrm{Cd}_{1 \cdot \mathrm{x}} \mathrm{Mrn}_{\mathrm{x}} \mathrm{Te}$, which offers sensitivities as great as three orders of magnitude above that of silica $[10,39,56]$. Additionally, a wavelengut of operation can be selected for a given Manganese concentration that results in temperature independent Faraday rotation [57]. Because of its crystalline structure, however, this material has yet to be adapted to current sensor applications. Devices have primarily been confined to small volume magnetic field sensors [10].

The largest Faraday cotations are produced in ferromagnetic (or fertimagnetic) crystals $[2,13,39]$. The most notable ferromagnetics are the iron garnets (e.g., YJG. yltrium iron gamet). In these malerials, an applied magnetic fueld aligns the magnetic dipoles of the individual domains. The specific rotation per unit applied field (equivalent 10 the Verdet constant in diamagnetic or paramagnetic materials) is the ratio of the saluration rotation, $\Theta_{F}^{\text {sol }}$, to the saturation magnetic field, $\mathrm{H}_{\text {sat }}$ This parameter is comparatively guite large, especially in the substituted iron garnets [12,58]. In $20 \%$ gallium substituled YhG (for the iron), Faraday sensitivity is reported to be $2.5 \times 10^{4}$ larger than in silica, which is approximately 20 times greater than in $\mathrm{Cd}_{-3} \mathrm{Mr}$ High sensitivity magnetic field sensors have been constucted using YIG [13] and Ga:YIG [12], and small rods of Ga:YIG have been arranged around a conductor in a corrent sensor configuration [30]. By the nature of the ferromagnetic material, saturation magnetization can be a limiting factor [58]. However, for the measurement of small fields or currents, for which these materials would be targeted, this saturation limit should not interfere.

Ultimately, the sensitivity limit for a Faraday effect current sensing systern will be established by noise levels. Sources of noise are fluctuations in optical power 
throughout the system and shot and thermal noise in the optical receiver. Laser noise sources and polarization variations due to temperature or stress vibrations can be large, but through careful component selection and system design, these fluctuations can be reduced significantly. The minimum measurable currents will then depend upon the receiver characteristics, in particular, the average optical detector output current, $i_{\text {det }}$, and the amplifier feedback resistance, $R_{\mathbf{f}}$ Al expression for the noise equivalent current, NEI, given per unit root bandwidth [59], is

$$
\mathrm{NEI}=\frac{\sqrt{\frac{2 \mathrm{k}_{\mathrm{B}} \mathrm{T}}{\mathrm{R}_{\mathrm{f}}}+e \mathrm{i}_{\mathrm{det}}}}{2 \mu_{\mathrm{f}} V N \mathrm{i}_{\text {det }}},
$$

where $\mathrm{K}_{\mathrm{B}}$ is Boltzmann's constant, $\mathrm{T}$ is the absolute temperature, $e$ is the magnitude of the electron. charge, and $\mathrm{N}$ is the number of turns in the fiber sensor coil. A plot of Eq. (2-10) for a singie tuin sensor coil with several values of the feedback resistor is shown in Fig. 2-5. For small values of the resistor, $R_{f}$, a slope of unity between NEI and the average detector current is approached, indicating dominant Johnson or themnal noise. For large resistor values, shot noise dominates and the curve approaches a constant slope of $1 / 2$ for all detector currents. It is expected that average detector output currents are not likely to exceed about $1 \mathrm{~mA}$, resulting in system NEIs of $\sim 1 \mathrm{~mA} / \sqrt{\mathrm{Hz}} / \mathrm{tum}$ for the most sensitive fiber sensor systems [45]. Further improvements in system response can only be gained through more turns in the fiber coil or through the use of higher Verdel constant materials.

\section{Sensor Bandividth}

The bandwidth of Faraday effect current sensors is limited by four basic factors:

(1) the intrinsic response of the Faraday rotator material; (2) the response time of the 


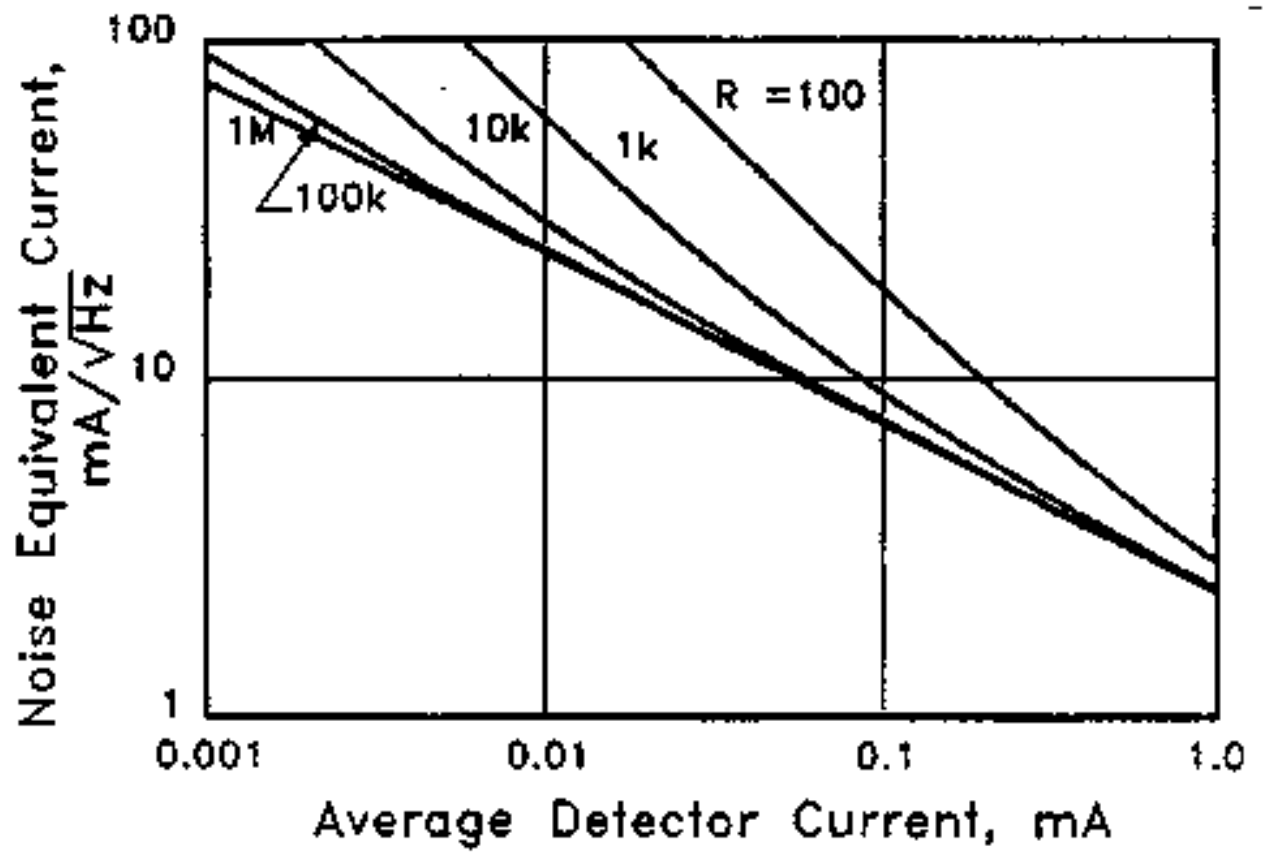

Fig. 2-5. The noise equivalent current per root unit bandwidth for a single turn fiber current sensor plotied as a function of the average detector output current. The effect of varying the foedback resistance in the transimpedance detection amplifier is shown.

pholodiode and the bandwidth of the amplifier used in the receiver circuit; (3) the dispersion of the propagating fight while passing through the sensing and transmitting medium; and (4) the transit time of the light propagating through the sensor materiaz. For mos1 sensoror systems, items (2) and (3) are of little concern. Photodiodes with a few tens of picoseconds of response time are available as are amplifiers with greater than 10 $\mathrm{GHz}$ bandwidths. And since relatively short lengths of singlemode fibers and high quality glasses or crystals are used in sensor systems along with laser sources, dispersion effects should not begin limiting bandwidths until tens of gigahertz.

The intrinsic response in Faraday rotator materials is determined by the relaxation or spin reorientation time of the interacting magnetic ions or domains. Each class of materials - diamagnetic, paramagnetic and ferromagnetic - can have a different microscopic interaction mechanism for the Faraday effect, resulting in effective Verdet 
constants that are widely distributed in frequency response. In some paramagnetic glasses and crystals the frequency dependent Faraday rotation, $\Theta_{m}(\omega)$, is described by a simple Debye relaxation function [37]

$$
\Theta_{m}(\omega)=\frac{\Theta_{m}(0)}{\sqrt{I+\omega^{2} \tau_{R}^{2}}},
$$

where $\theta_{m}(0)$ is the zero frequency response of the material, $\omega=2 \pi i$ is the angular modulation frequency of the magnetic field, and $\tau_{R}$ is the material relaxation time conslant. This model has been compared to measurements made on $\mathrm{Cd}_{1-\alpha} \mathrm{Mn}_{\mathrm{x}} \mathrm{Te}$ sensors [38]. The frequency dependent Faraday rotation (effective Verdel constant) for the $\mathrm{Cd}_{1-x} \mathrm{Mn}_{\mathrm{x}} \mathrm{Te}$ system is shown in Fig. 2-6 for two different compasitions. Different laser wavelengths were required for maximum Faraday response since the bandgap changes with composition. When the Manganese concentration is increased from $10 \%$ to $45 \%$, the relaxation time decreases, resulting in nearly an order of magnitude increase in the frequency response.

In ferromagnetic materials, the frequency dependence of the Faraday effect is also highly dependent on composition $[30,31]$. Typically, the larger the enhancement of the localized magnetic field, the slower the movement of the domain watls. In Fig. 2-7, the frequency response is ploted for samples of pure YIG [13] and Ga:YIG [30]. The YIG sample has a $-3 \mathrm{~dB}$ frequency of approximately $700 \mathrm{MHz}$, while the Ga:YIG sample has a bandwidth of $\sim 7.5 \mathrm{MHz}$. These frequency responses scale inversely (though not linearly) with the increases in their relative Faraday sensitivities; the Ga:YIG has a significantly larger rotation per unit field.

In diamagnetic and paramagnetic glasses, the magnetic ion retaxation times are usually less than one picosecond [37]. Thus, in fiber current sensor systems where the singlemode fiber has been drawn from common glasses or glasses doped with magnetic 


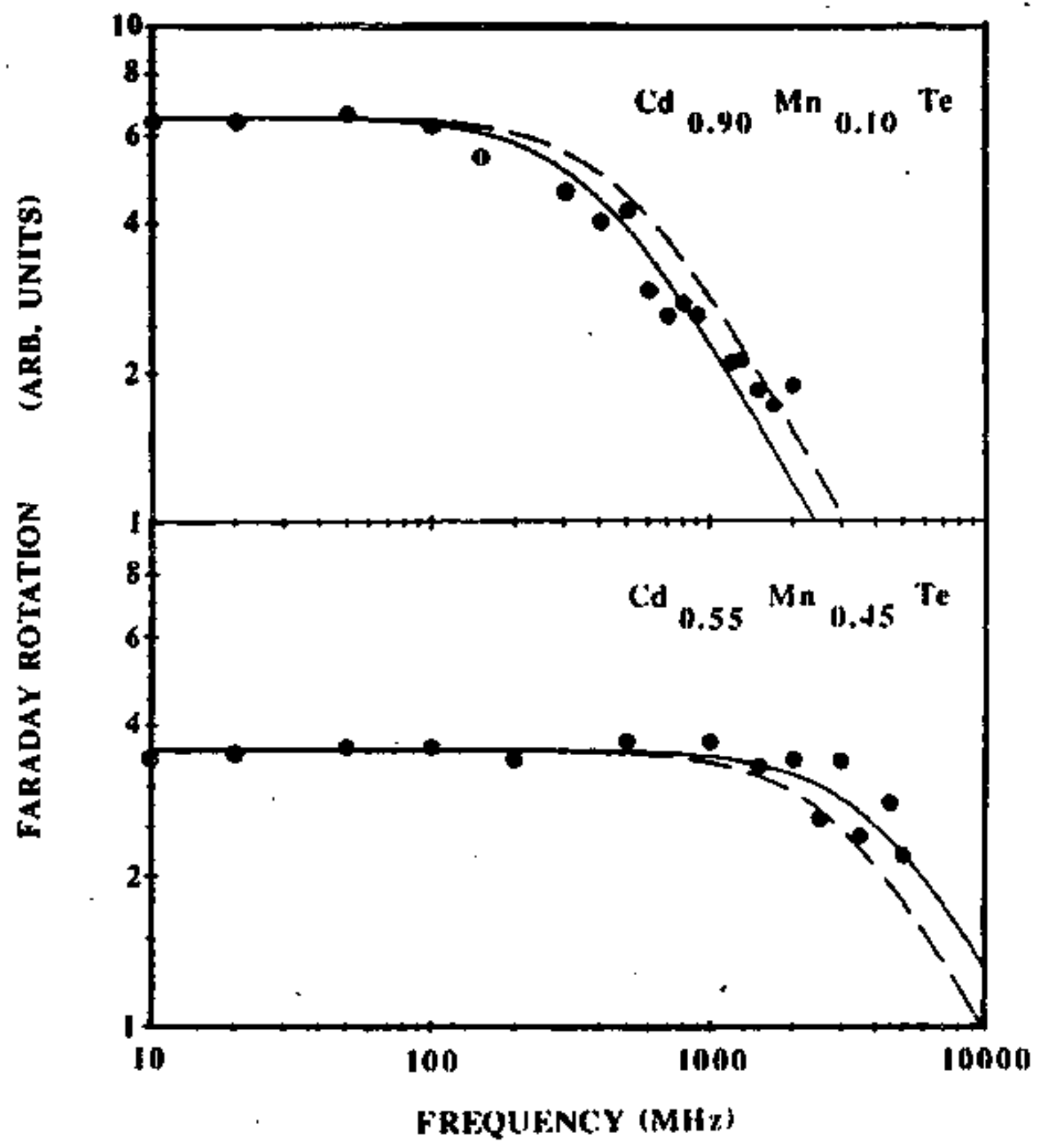

Fig. 2-6. Relative changes in the Faraday rotation versus frequency for two Manganese concentrations in $\mathrm{Cd}_{\mathrm{l}_{-}} \mathrm{Mn}_{\mathrm{x}} \mathrm{Te}$ [38]. Each set of data was taken using a laser wavelength optimized for material response with that Mn concentration. Solid and dashed lines represent theoretical fits to the frequency dependence. 

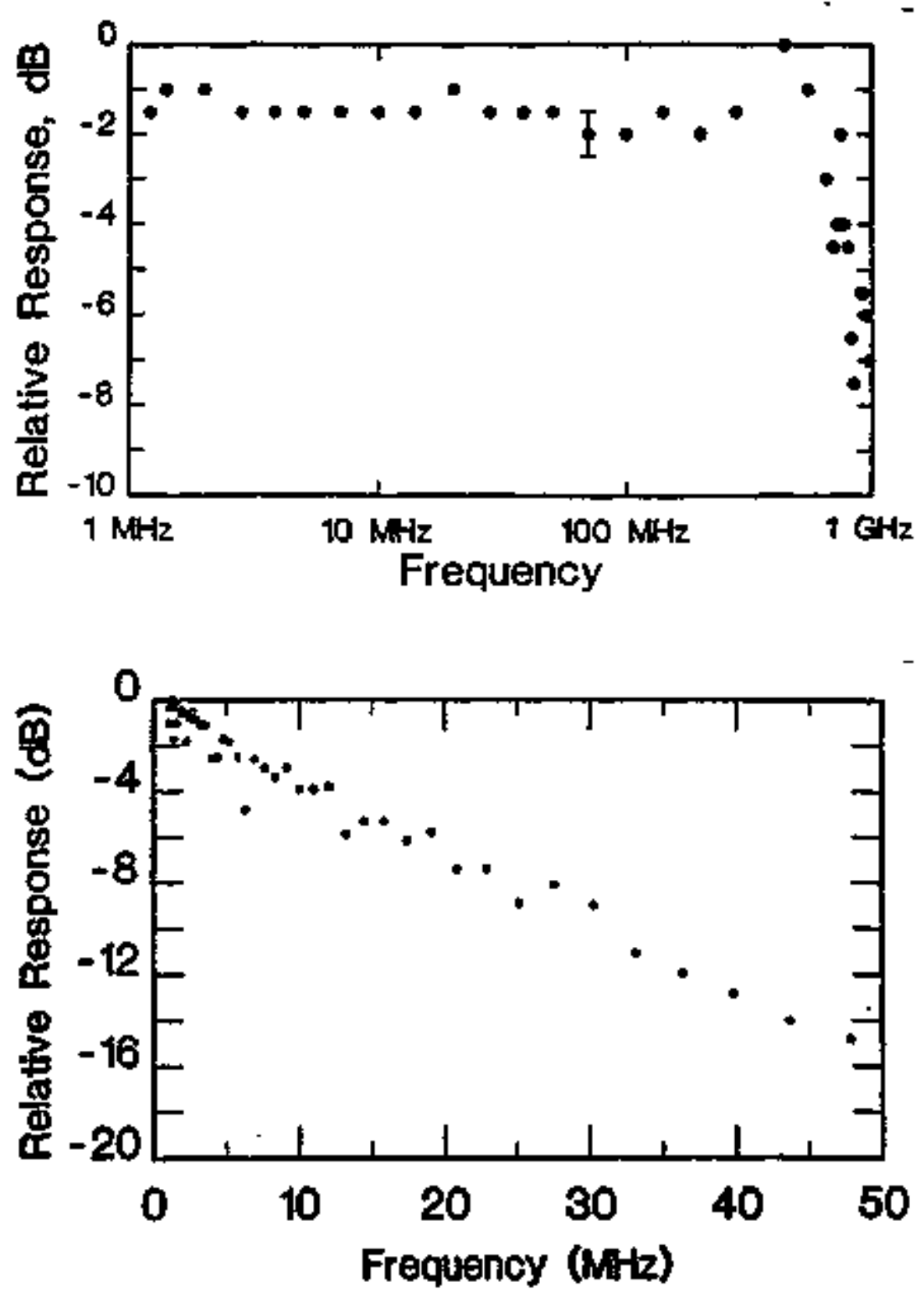

Fig. 2-7. The frequency response of the Faraday rotation for two compositions of YIG crystals. (Top) A $5 \mathrm{~mm}$ diameter by $3 \mathrm{~mm}$ long sample of pure YIG [13]. (Bottom) A $1.5 \mathrm{~mm}$ diameter by $5 \mathrm{~mm}$ Iong sample of Ga:YIG [30]. 
ions, it is safe to assume that intrinsic material freguency response will not be the limiting factor in system bandwidth. In most practical applications, the bandwidih will be constrained by the light transit time through the sensing material. This is particularly toue for fiber sensors in which many turns have been used in a coil for increasing measurement sensitivity. In order for the rotation to be described by Eq. (2-3), the amplitude variations in the conducted current (or the associated magnetic field) must be smatl during the time it takes for the light to propagate from one end of the fiber coil to the other. Otherwise, changes in the field become convolved with the propagating light rotations. The dependence of fiber current sensor frequency response on the light transit time and the subsequent limitations on bandwidth are the subject of this study. Theoretical and experimental descriptions of the frequency dependent Faraday rotation for many cunent sensor coniggurations appear throughout the remainder of this document. 


\section{CHAPTER 3}

\section{TRANSIT TIME FREQUENCY RESPONSE THEORY}

In order to derive an expression for the frequency dependent Faraday rotation, three assumptions are made about the optical fiber current measurement system: (1) the circular birefringence produced by the Faraday effect dominates the pojarization state in the fiber; (2) the magnetic field propagating along with the current on the conductor is planar and azimuthally symmetric - in general, a transverse electromagnetic (TEM) wave: and (3) the current flowing along the conductor is treated as an infinitely long filament of moving charge centered at the conductor's longitudinal axis. The first assumption implies that any significant linear birefringence in the fiber has been removed through annealing or twisting so that uncharacteristic polarization shifts in the fiber are not convolved with the remaining electromagnetic interactions. And the last two assumptions assure that all points along the fiber that fall in a plane perpendicular to the conductor experience simulianeous exposure to the passing magnetic wave.

The magnetic flux density from the passing TEM wave has a spatial and temporal dependence given by

$$
B(r, t)=\frac{\mu_{\Gamma} \dot{i}}{2 \pi r} e^{-j(\omega t \cdot k \cdot l)} \hat{\phi},
$$

where $r$ is the radial distance from the conductor to the light element propagating in the fiber, $\omega=2 \pi f$ is the angular modulation frequency of the freld, $k$ is the propagation vector of the TEM wave pointing in a direction parallel to the conductor, $I$ is the length vector for the fiber coil, and $\hat{\phi}$ is the unit vector in the azimuthal direction. In Eq. (3-1) the current, $i$, is taken to be static, or slowly varying with respect to $\omega$. The fiber coil and its position relative to the conductor is allowed to be in many configurations. In general, 
the coil will not have a circular cross section, the conductor will not be centered in the coil cross section, and the coil turns will not be collocated (allowed to form a helix). Thus. B and dl in Eg. (2-1) will not be codirectional as is the case for a circular coil with a centered conductor, and the vectors $k$ and I in Eg. (3-1) will not be orthogonal as with a coil with collocated tums.

The angles formed between the field vectors and the light elements in the fiber are illusirated in Fig. 3-1. B and dl subtend an angle $\gamma$ and $\mathbf{k}$ and dl subtend an angle $\psi$, so that

$$
B * \text { dl }=B \cos \gamma \sin \psi d i
$$

and

$$
\mathrm{k} \cdot \mathfrak{l}=\mathrm{kj} \cos \psi
$$

Substituling the information from Egs. (3-1) through (3-3) inco Eq. (2-1) results in an expression for the frequency dependent Faraday rotation:

$$
\theta(\omega)=\frac{\mu_{\mathrm{f}} \mathrm{Vi}_{\mathrm{i}}}{2 \pi} \int_{0}^{\mathrm{L}} \frac{1}{\mathrm{r}} \cos \gamma \sin \psi \mathrm{e}^{-\mathrm{j}(\omega \mathrm{t}-\mathrm{kJ} \cos \psi)} \mathrm{d} \mathrm{l}
$$

The wave propagation constant can be expressed as $k=\alpha v_{p h}$, where $v_{p h}$ is the wave phase velocity (usualiy the speed of light in the medium surrounding the conductor). The surrounding medium totally innerses the frber and may be a materiaj other than air or vacuum. In general, the medium will have permittivity of $\varepsilon$ and permeability of $\mu$. Then the phase velocity is given by 


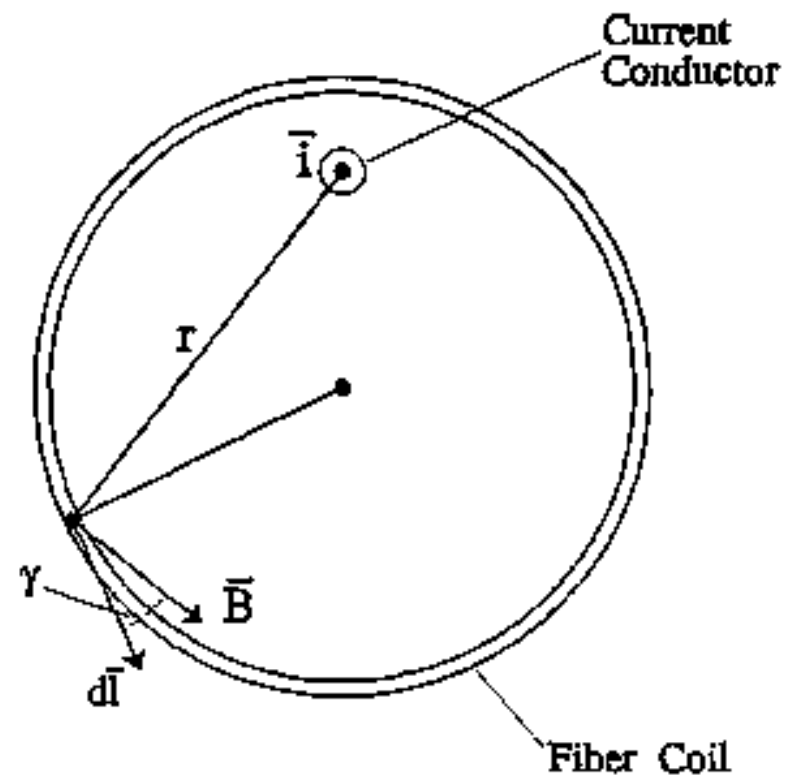

(a) Side View

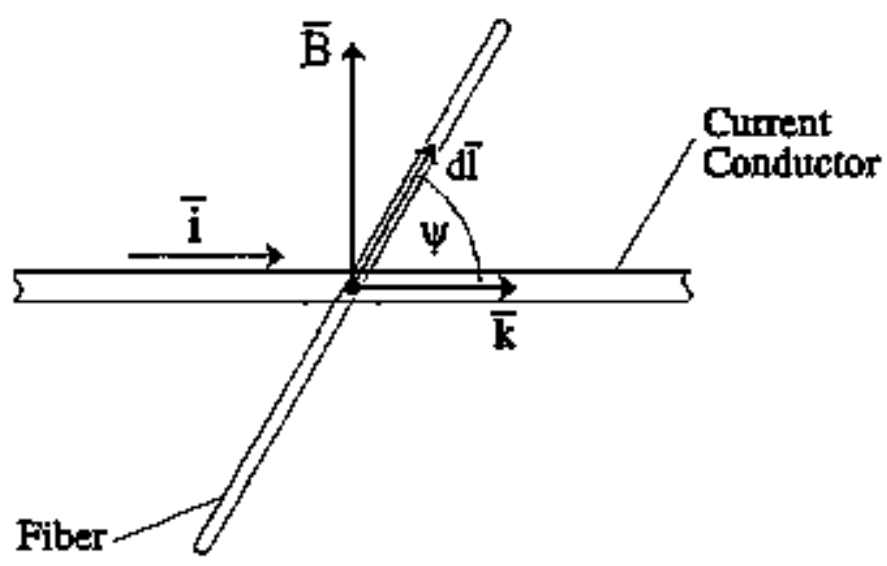

(b) Top View

Fig. 3-1. Angles formed between the light propagating in the fiber coil and the magnetic field associated with the condacted current. 


$$
v_{p h}=\frac{1}{\sqrt{12 \mathrm{E}}}
$$

Since $\varepsilon=\varepsilon_{0} \varepsilon_{\mathrm{r}}$ and $\mu=\mu_{0} \mu_{\mathrm{r}}$, where $\varepsilon_{0}$ and $\mu_{0}$ are the free space permituvity and permeability, and $\varepsilon_{\gamma}$ and $\mu_{\mathrm{r}}$ are the material relative permittivity and perneability, the phase velocity can be rewritten as

$$
v_{p h}=\frac{1}{\sqrt{\mu_{0} \varepsilon_{0}}} \frac{1}{\sqrt{\mu_{r} \varepsilon_{r}}}=\frac{c}{\sqrt{\mu_{r} \varepsilon_{s}}}
$$

with $\mathrm{c}$ being the speed of light in a vacuum.

As the light in the fiber propagares through the coil, $r, \gamma$ and $\psi$ in Eq. (3-4) can vary, making them tine or position, dependent parameters. Equation (3-4) is changed from a jength integral to a time inlegral, using the relationship $\mathrm{l}=(\mathrm{c} / \mathrm{n}) \mathrm{t}$, where $\mathrm{n}$ is the fiber refraclive index. The expression for $k$, incorporaling Eq. (3-6), and the time dependencies of all parameters are substituted into Eq. (3-4) to give

$$
\left.\Theta(\omega)=\Theta_{0} \frac{1}{2 \pi N}\left(\frac{c}{n}\right) \int_{0}^{\tau} \frac{\cos [\gamma(())] \sin [\psi(t)]}{r(t)} e^{-j \omega t\left[1-\frac{\sqrt{\left[\mu_{T} \varepsilon_{\Gamma}\right.}}{n} \cos [\psi(1)]\right.}\right] d t
$$

where $\tau=(n / \mathrm{c}) \mathrm{L}$ is the transit time of the light through the fiber. In Eq. (3-7), the low frequency Faraday rotation, $\theta_{0}$, has been introduced through substitution of Eq. (2-3). The frequency dependent Faraday rotation as expressed by Eq. (3-7) is then a function of the position of the current-carrying conductor relative to the fiber coil, the coil shape (cross section and helical parameters), and the relative phase between the magnetic field and the light propagating in the fiber.

For a given current measurement system, Eq. (3-7) can be solved to predict the 
Faraday rotation freguency response. Solutions for particular system geometries are presented in the following chapters of this document. Two simplifications of Eq- (3-7) are of greatest interest in providing the starting foundation for those developments. First is the case where the fiber coil tums are collocated and the plane of the coil is perpendicutar to the conductor. In this configuration all points along the fiber coil are simultaneously exposed to the passing magnetic field. With $\psi=90^{\circ}$, the expression for the frequency dependen Faraday rotation becomes [14]

$$
\Theta(\omega)=\Theta_{0} \frac{1}{2 \pi N}\left(\frac{c}{n}\right) \int_{0}^{\pi} \frac{\cos [\gamma(t)]}{r(t)} e^{-j \omega t} d t
$$

Sensor coils having this planar geometry are discussed in detail in Chapters 4 through 7 .

The second case fixes the conductor at the center of a circular cross section coil, but allows the coil tums to be spread apart. This configuration represents a travelling wave geometry that increases the phase matching between the propagaling light in the fiber and the passing electromagnetic wave [60]. With the conducior centered, $\mathrm{r}=\mathrm{R}$ (fixed radius of the circular coil), and $\gamma=0$. Then Eq. (3-7) reduces to

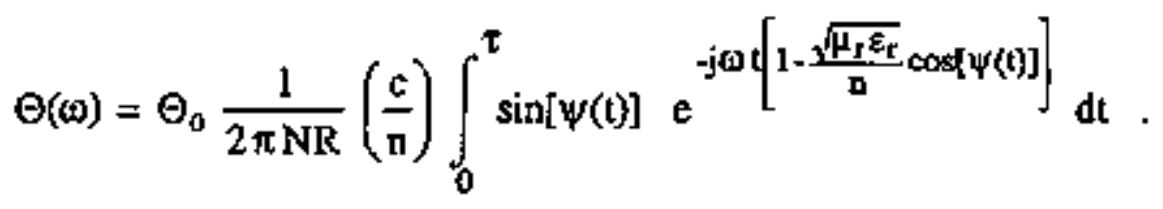

Travelling wave fiber current sensors are presented in Chapters 8 through 11.

Throughout this paper, particular interest is generated from the magnitude of the Faraday response relative to its low frequency value, $\left|\Theta(\omega) / \Theta_{0}\right|$. The frequency dependent response can be calculated using either Eq. (3-8) or Eq. (3-9), or directly from Eq. (3-7) if more complex configurations wish to be explored. At frequencies near zero 
(static or d.c. conditions), the relative magnitude is always unity. At the higher frequencies, this magnitude will rolloff, but in general, it can have values anywhere between zero and one at any given frequency. A frequency, $\omega_{v}$, is defined at the poin where the relative magnitude is first reduced from its static value to

$$
\left|\frac{\Theta\left(\omega_{u}\right)}{\Theta_{0}}\right|=\frac{1}{\sqrt{2}}
$$

This frequency provides the $3 \mathrm{~dB}$ bandwidth, $\Delta \mathrm{B}$, for the current measurement system under investigalion. The bandwidth is a useful parameter for comparing the response and performance of systems that use different types of optical fiber coils. In general, the bandwidlhs are strong functions of the transil time, $\tau$. A second parameter of interest is the cutoff frequency, $\omega_{c o}=2 \pi \mathrm{f}_{\mathrm{co}}$. which is defined as that frequency where the Faraday response first equals zero. Multiple nulls and high frequency tobes of the Faraday rolation response occur for many of the fiber systems studied here.

The assumption that a TEM wave surrounds the current carrier is usefol in simplifying the theorelical development needed to model fiber current measurement systems and providing a framework within which an experimental verification is possibte. A single wire conductor produces non-TEM fields consisling of many modes and exhibiting strong spatial distributions [61]. Wave impedances vary with frequency, and a simple relationship between currents and fields may not be possible to determine. This obviously would introduce additional complexity into the Faraday response computations of Eqs. (3-7) through (3-9). A coaxial transmission line, however, produces a magnetic flux density given by Eq. (3-1) and satisfies the TEM condition. The fiber current sensor models developed in this study do not attempt to treat the additional high frequency effects produced by the single wire conductor; that is left for future study. 


\section{CHAPTER 4 \\ EXPERMENTAL APPARATUS AND PROCEDURES}

The instrumentation used in the high frequency measuremerts of fiber current sensor coils is shown in the schematic of Fig. 4-1. Linearly polarized light from a laser source passes through bean expansion optics and a half-wave retarder, then is injected into a singlemode fiber lead using a 10x microscope objective. Light in the fiber lead is

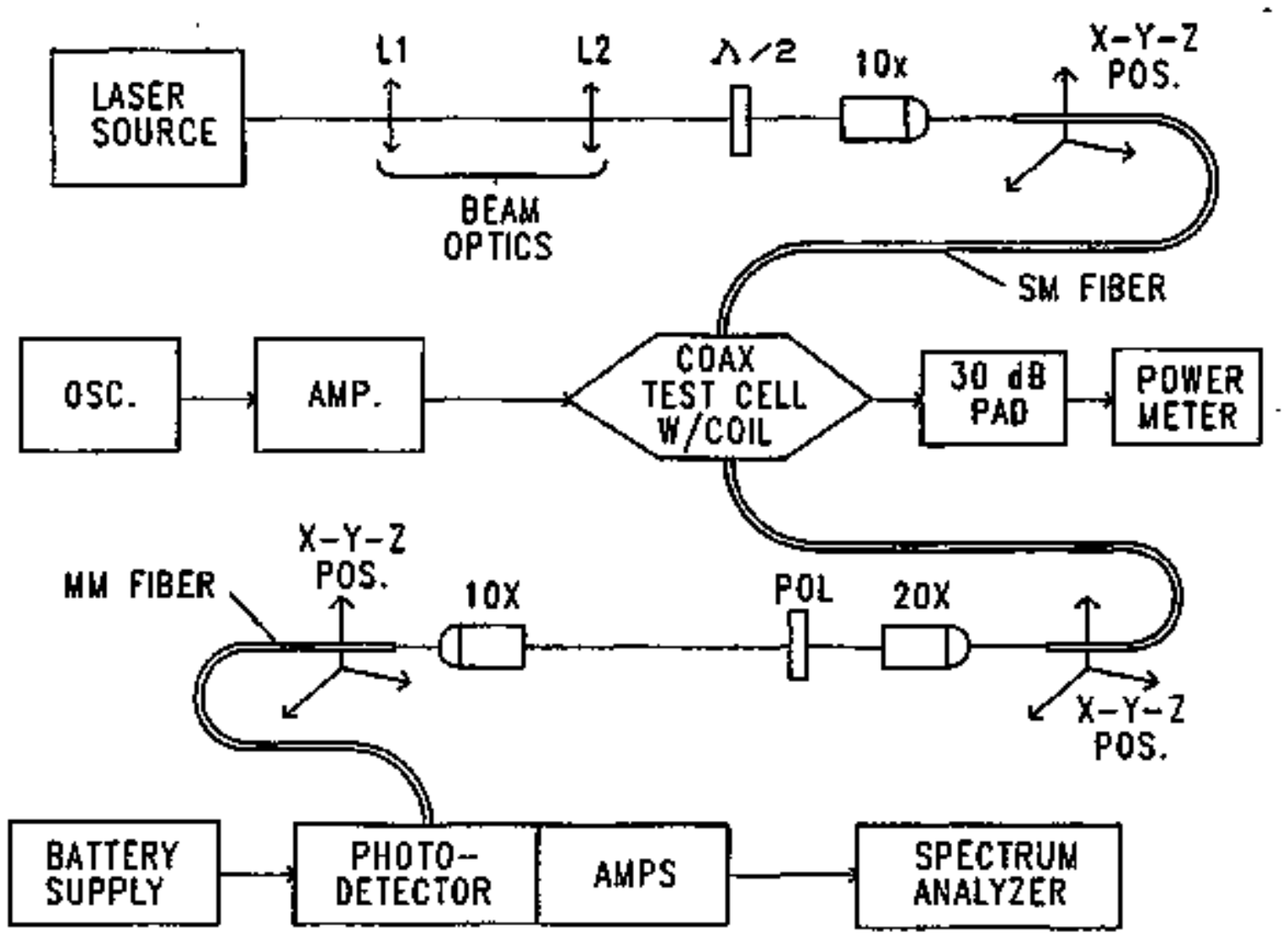

Fig. 4-1. Arrangement used for measuring the frequency response of the Faraday effect in optical fiber current sensor coils. L1 and L2 are lenses used for beam expansion and collimation, $\lambda / 2$ is a double Fresnel rhomb half-wave plate, and the components marked by $10 \mathrm{X}$ and $20 \mathrm{X}$ are microscope objectives tused for light injection into the optical fibers and collimation of light exiting from the fibers. 
carried to the sensor coil located inside a special coaxial test cell. The light undergoes a rotation (induced by the conducted current), is transported out of the cell via the output fitber coil lead, and then exits the fiber. The polarization is analyzed, and the resultant optical signal is reinjected into a multimode fiber leading to a high speed photodiode. Signals are amplified and their magnitudes are displayed on a spectrum analyzer. Three different laser sources were used during experimentation: a He-Ne laser (633 nm, $8 \mathrm{~mW}$ ) and two GaAlAs diode lasers $(780 \mathrm{~nm}, 3 \mathrm{~mW}$ and $840 \mathrm{~nm}, 30 \mathrm{~mW}$ ). Figure $4-2$ is a bench-top photograph of the optical components used in the experimental arrangement

Single frequency RF signals were generated by an oscillator or synthesized signal generator and then amplified to a maximum of 10 Watts before injection into the coaxial test cell. The power transmitted through the test cell was aftenuated (usually $30 \mathrm{~dB}$ ) and monitored by a power meter. All the RF instrumentation in the drive line operates into a $50 \Omega$ impedance, thus producing a maximum ms test current of approximately $0.45 \mathrm{~A}$.

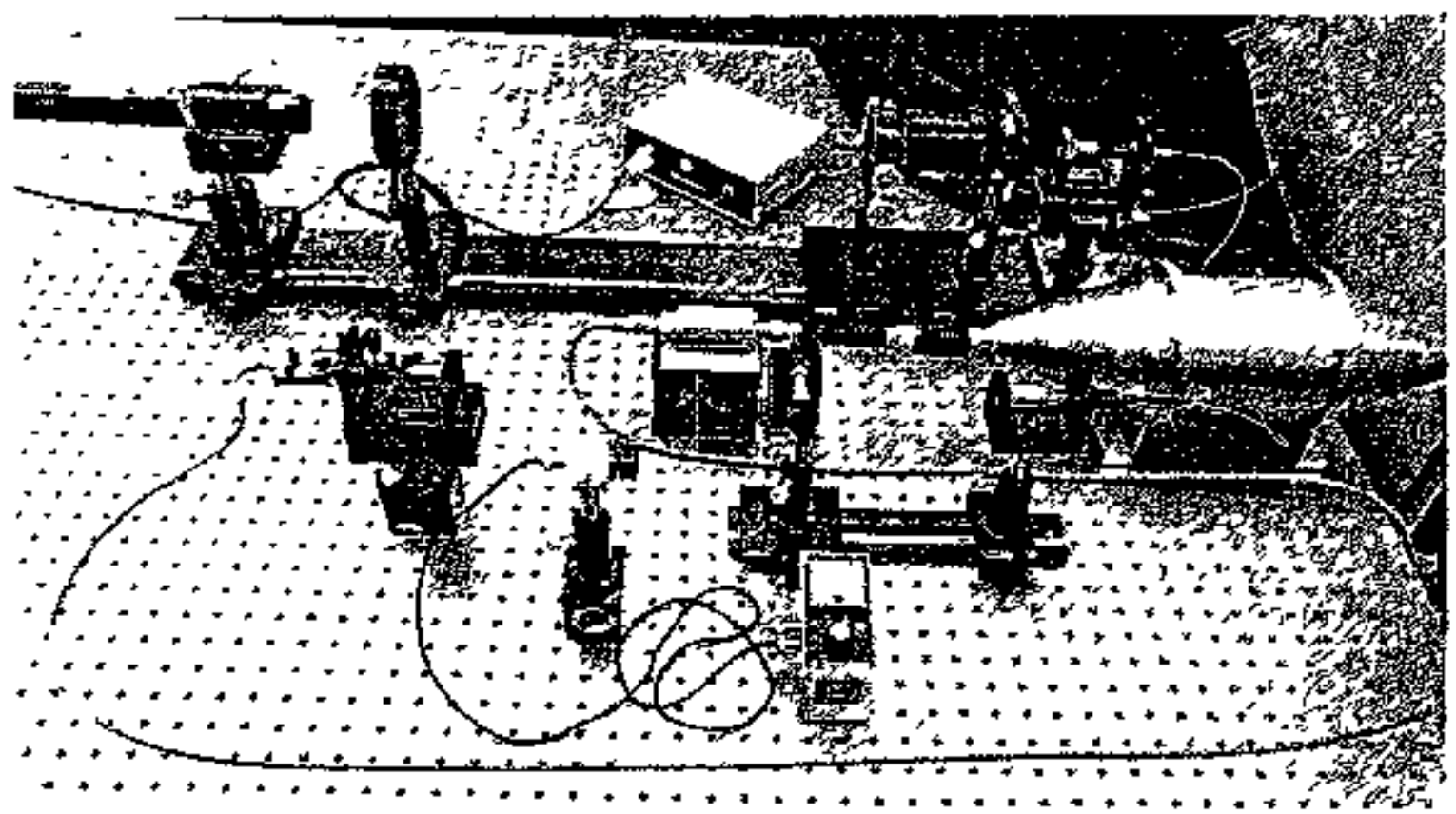

Fig. 4-2. A photograph of the optical components on the bench top used in the measurement of fiber current sensor response. 
The general frequency range covered doring experimentgtion was $500 \mathrm{kHz}$ to $1 \mathrm{GHz}$. The photograph in Fig. 4-3 shows the RF instrumentation used: signal generators, highpower amplifiers, power meter, photodetectors and spectrum analyzers.

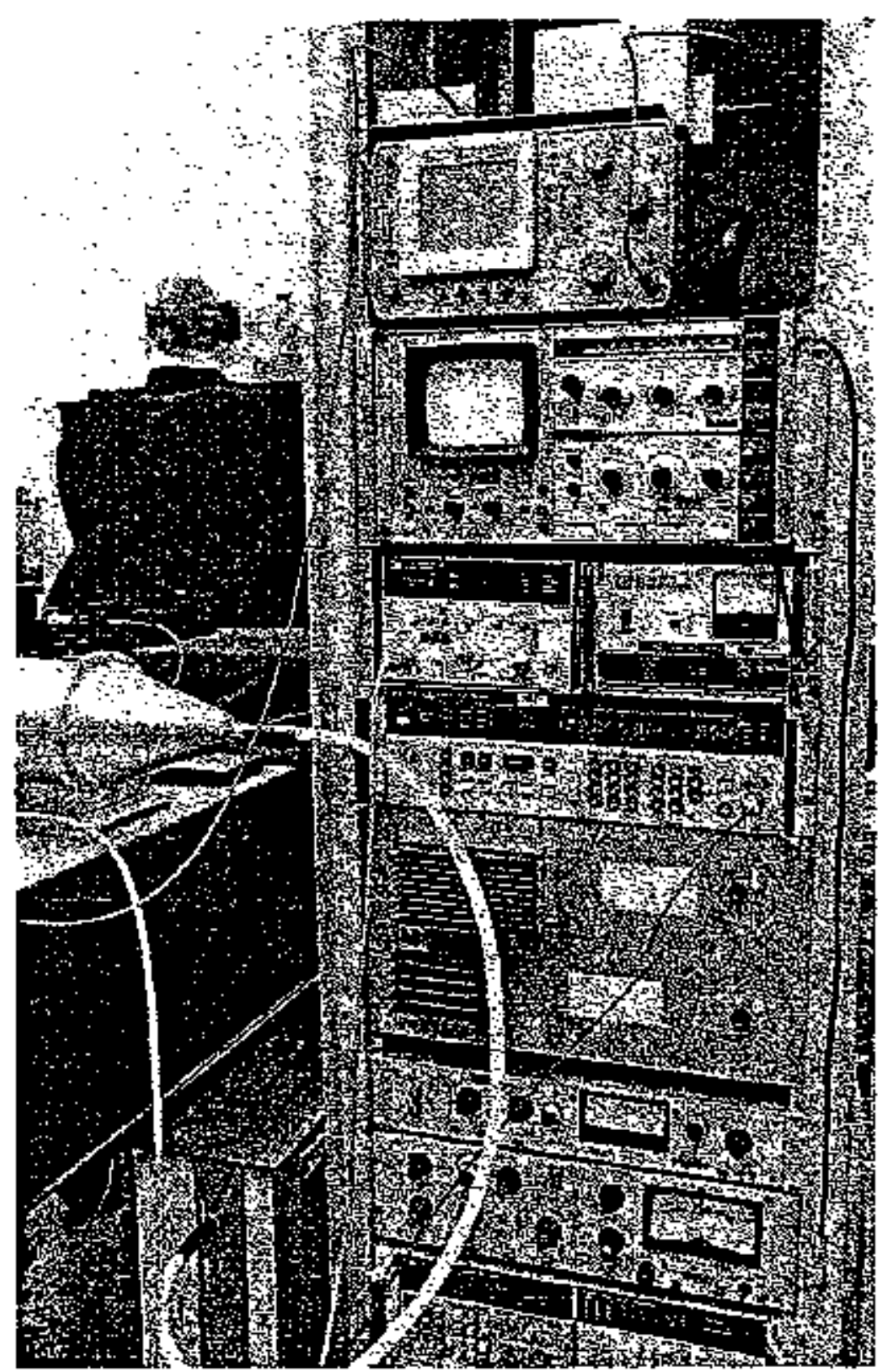

Fig. 4-3. The RF drive and measurement instrumentation used in characterizing the response of fiber current sensor coils. 
One of three high speed photodiodes was used at the front end of the photodetection system. These include a silicon PIN photodiode, a silicon avalanche photodiode (APD) and a GaAiAs photodiode. Each has a response bandwidth in excess of $1 \mathrm{GHz}$. The diodes along with bias circuitry and small-signal RF amplifiers were housed in shielded enclosures to reduce interference from the high power RF drive sources.

In Fig. 4-4, the schematic of the silicon PIN photodetection system is shown. The system includes $42 \mathrm{~dB}$ of amplification and a de bias port for monitoring light intensities during setup and alignrnent. $\mathrm{A} 1.2 \mathrm{AH}$ rechargeable battery pack served as the $+15 \mathrm{Vdc}$ supply. The frequency response of this photodetector is plotted in Fig. 4-5. In generating this response curve, an Ontel high speed GaAlAs semiconductor laser drove the input to the silicon PIN photodiode. Detector response does not deviate greater thant $2 \mathrm{~dB}$ for frequencies below - $1.1 \mathrm{GHz}$; however, the oscillations above $500 \mathrm{MHz}$ are

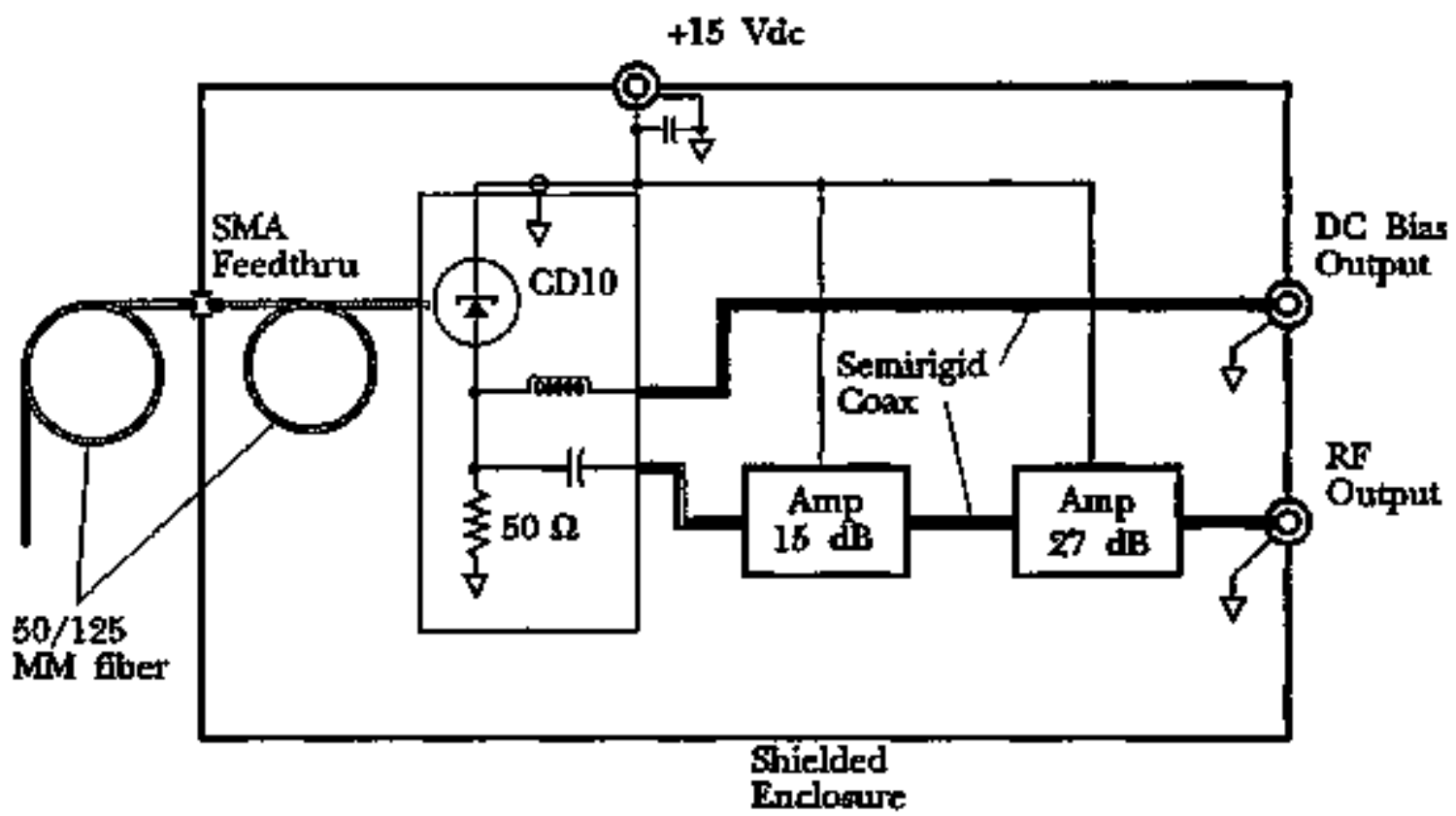

Fig. 4-4. Silicon PIN photodetection system built into a shielded enclosure. 


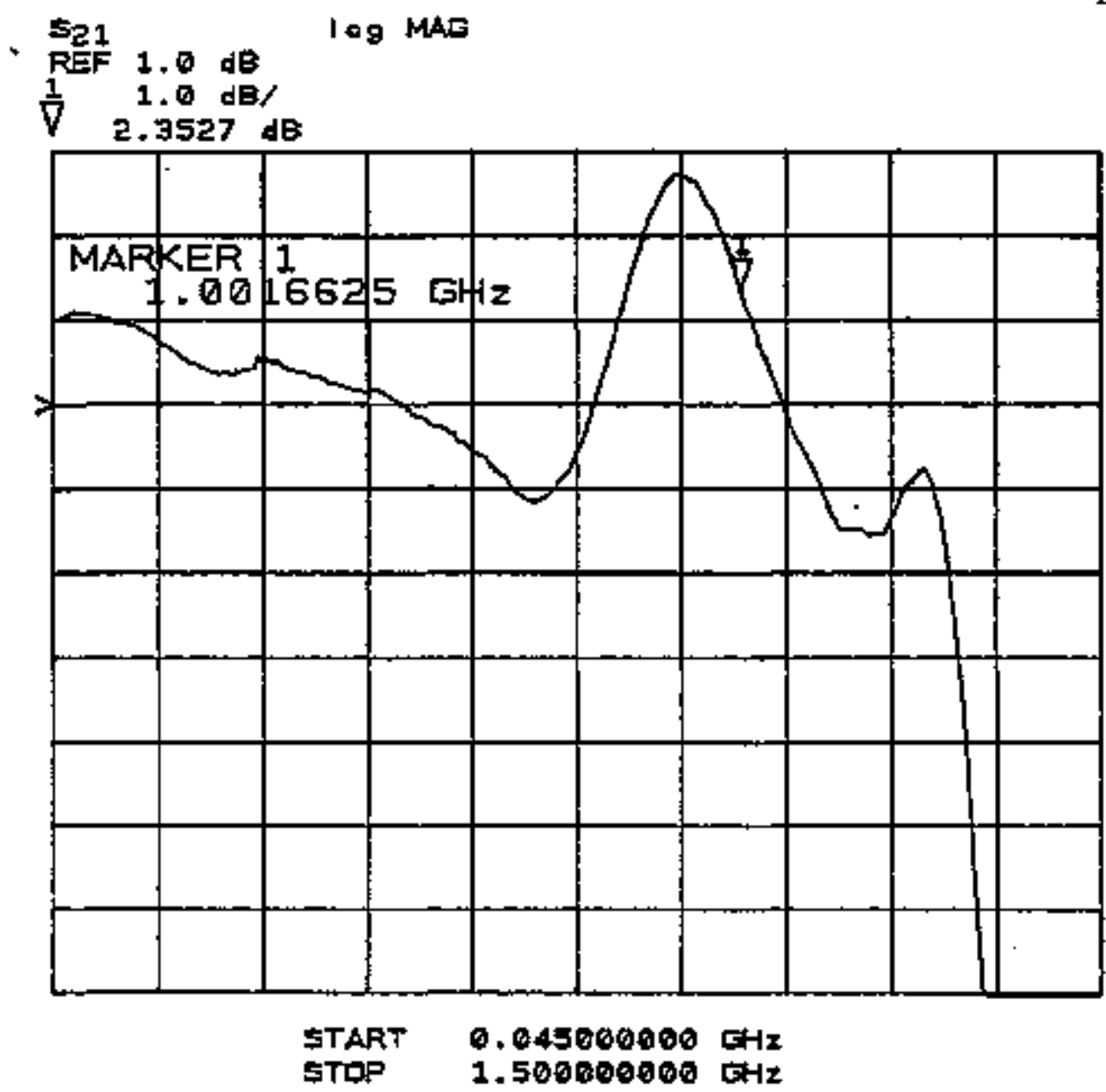

Fig. 4-5. The frequency response of the silicon PIN photodiode with $42 \mathrm{~dB}$ of serial, small-signal RF amplification.

large enough to warrant corrections in the measured cunent sensor data. The large response increase near $1 \mathrm{GHz}$ is caused by resonant amplification between the photodiode circuit and the first RF amplifier. This anomaly does not occur in all the photodetector systems.

The coaxial test cells are expanded transmission line chambers that provide near Ideal electromagnetic environments that impinge on the fiber coils [34]. Discussions of pertinent coaxial line properties and the characteristics of fabricated airlines are given in Appendix A. A cross sectional view of one of the test cells is presented in Fig. 4-6, and a 


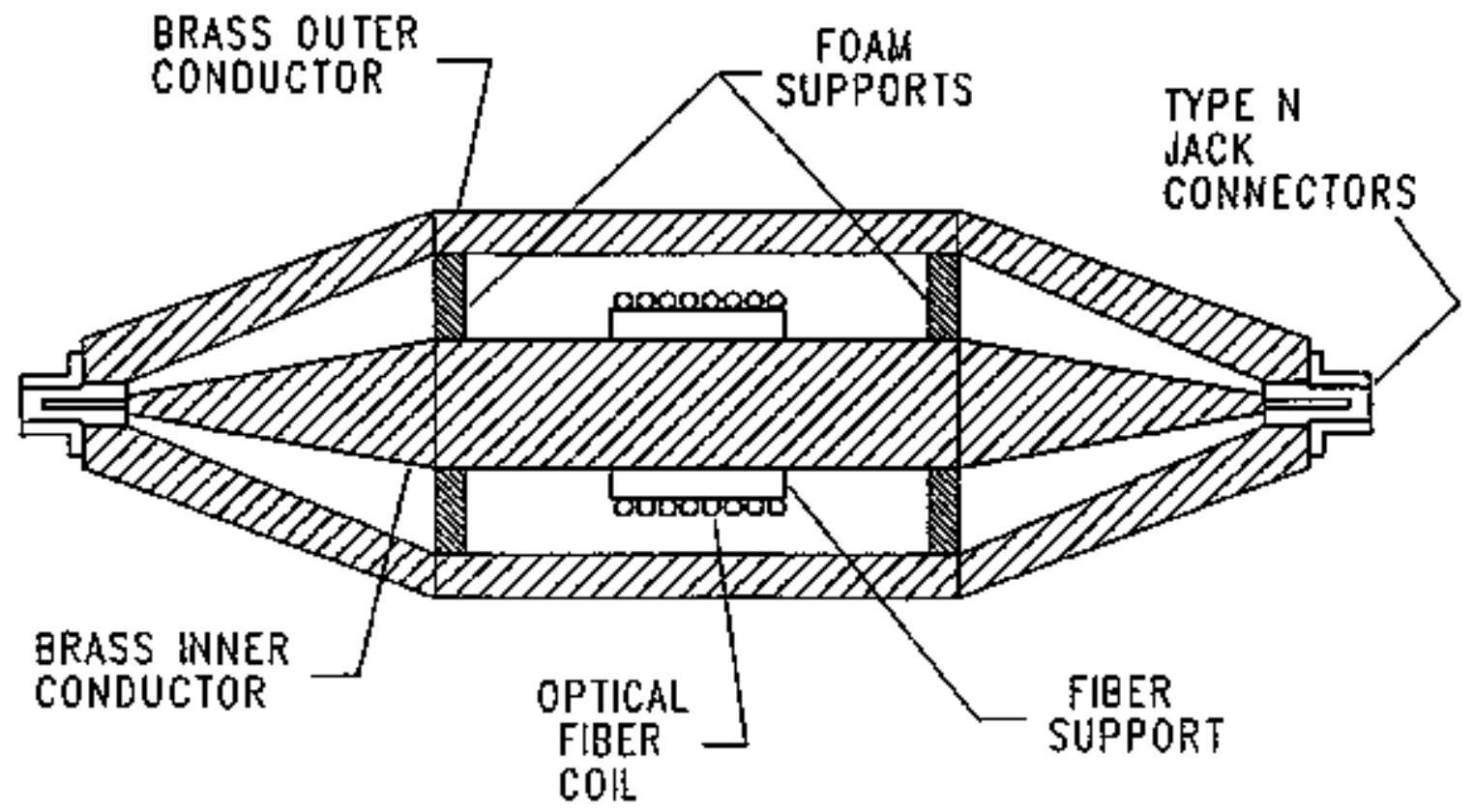

Fig. 4-ir. A cross sectional view of an expanded coaxial airline test cell with an optical fiber coil nounted in the working volume.

close-up ploolograph of the cell is shown in Fig. 4-7a. The cells are designed to open easily for mounting of the optical fiber coils in the cell working volume. The position of the coil inside the cell is seen in Fig. 4-7b. A transverse electromagnetic (TEM) wave is launched in the air space in the cell, and travels with a phase velocity equal to the speed of light. Cell impedance is designed for $50 \Omega$, requiring the ouler to inner conductor diameter ratio to be 2.3 . The conductor diameters also dictate the highest freguency to which TEM mode operation can be suslained. Three cells with higher-order mode clitoff frequencies of $1.0,2.3$ and $13.7 \mathrm{GHz}$ were used in these measurements; selection among these devices was dependent on the fiber sensor coit size.

All the cells were characterized with the fiber coils and any additional support structures mounted inside. Measurements of the standing wave ratios (SWR) and impedances of the cells indicate that electromagnetic properties varied minimally from the design. The $1-\mathrm{GHz}$ airline test cell was used most frequently during experimentation; 


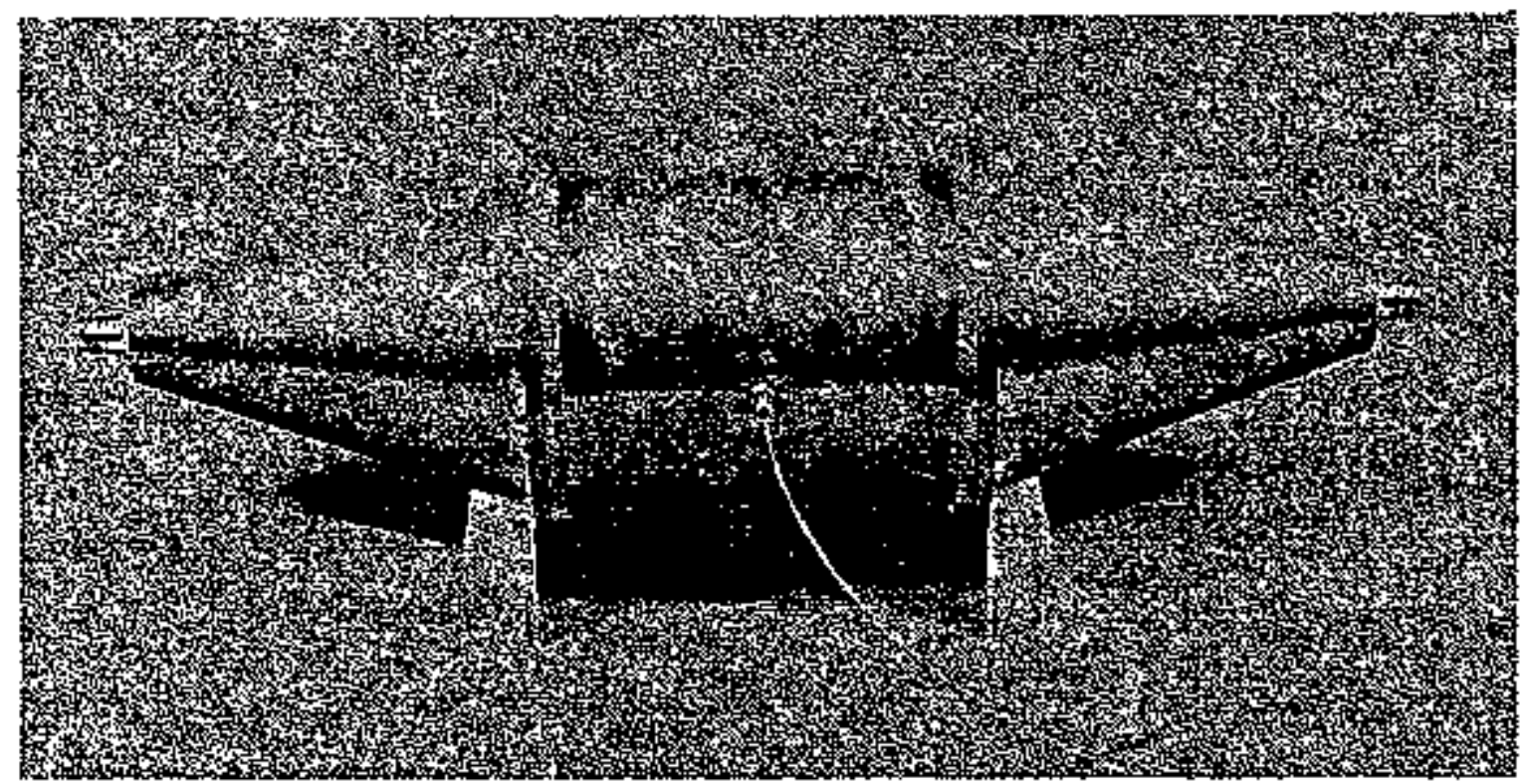

(a)

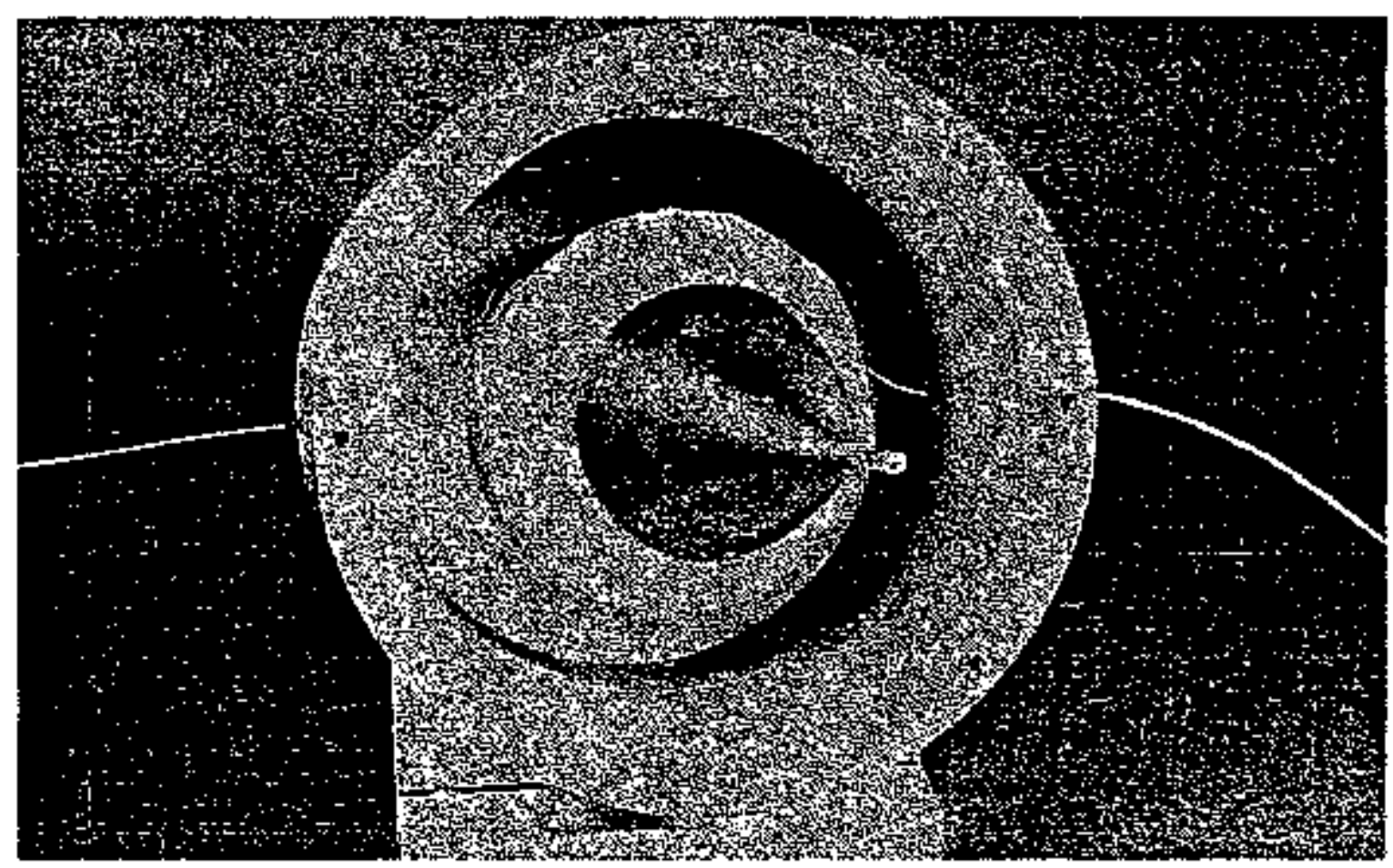

(b)

Fig. 4-7. Photographs of the 1-GHz expanded coaxial test cell. (a) The fully assembled cell, and (b) the opened cell showing a fiber coil mounted around the center conductor. 
its SWR and impedance magnitude are plotted in Fig. 4-8. Below $1 \mathrm{GHz}$, the maximum cell SWR is approximately 1.21 , and the impedance varies no more than $7 \Omega$ from its $50 \Omega$ nominal value ( $0.7 \mathrm{~dB}$ maximum deviation). These excellent cell impedances allow for the simple relationship between power and current, $P=i^{2} Z$, to be vtilized. Thus, only power monitoring was needed during coil testing to determine the relative magnitude of the sensed currents. The maximum enror in estimating the currents was always less than $0.7 \mathrm{~dB}$, and corrections for impedance deviations at individual frequencies coutd be applied where greater precision was required.

The coaxial transmission cells were also configured in non-airline modes with liguid dielectrics. These configurations are used in conjunction with travelling wave current sensor concepts and are discussed in detail in Chapter 9.

Optical fiber coils were constructed from low birefringence singlemode fiber with an $80 \mu m$ cladding diameter. In order to overcome the effects of bend-induced linear birefringence [50], fiber twisting [52] or fiber annegling [21] was eraployed during coil fabrication. For most of the sensors, a twisted and rejacketed fiber was used [29]. This fiber has an outer buffer diameter of $1 \mathrm{~mm}$ and can have twist rates as large as 40 turns/meter. With this fiber, coil constroction is greatly sinplified. Fiber coils were mounted approximately in the center of the test volume of the expanded coaxial test cells as illustrated in Figs. 4-6 and 4-7b. Some of the circular cross section coils were wound directly on the cell center conductor or were wound in a self-supportiag form using the twisted and rejacketed fiber. Other circular colls were supported using machined polyurethane foam $\left(10 \mathrm{lb} / \mathrm{ft}^{3}\right)$ or a polycarbonate. Fibers entered and exited the cell volume through the outer conductive wall (see Fig. 4-7a).

Using the half-wave retarder, the linear polarization angle of the light injected into the singlemode fiber was aligned with one of the fiber coil linear birefringence axes. Orientation of the axes (in all but the annealed fiber coils) are established by bend birefringence and twist-induced circular birefringence. The output polarizer/analyzer 


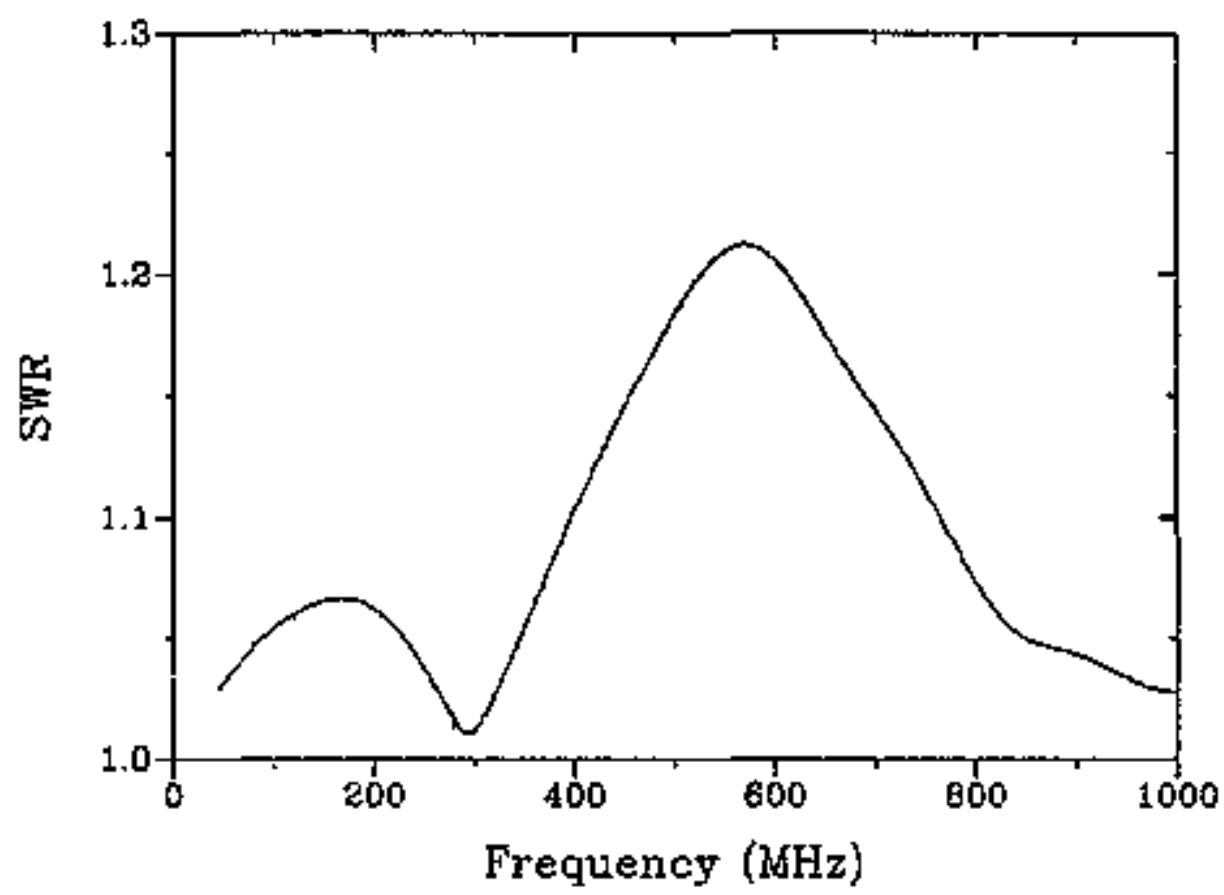

(a)

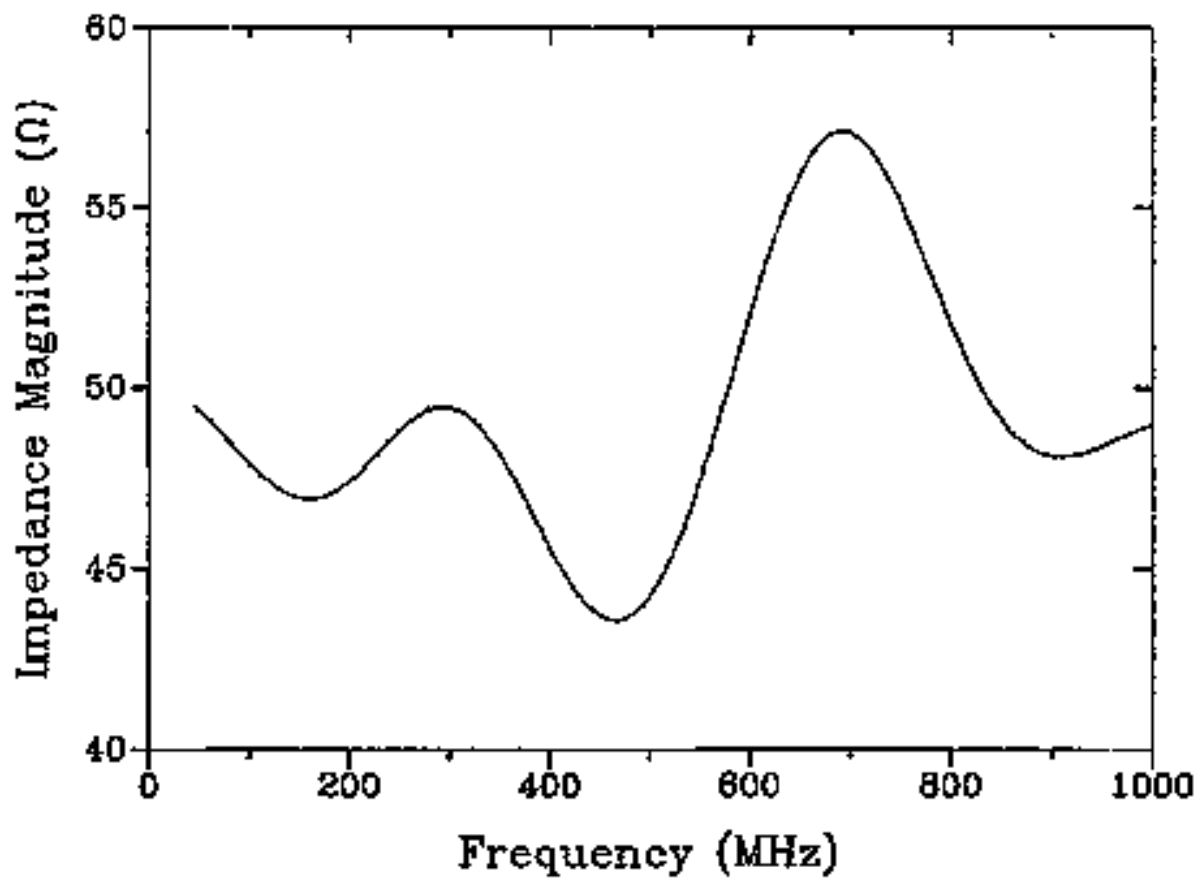

(b)

Fig. 4-8. The SWR (a) and the inpedance magnitude (b) for the 1-GHz cogxial test cell with an optical fiber coil inside. 
was set at $45^{\circ}$ to the exiting linear polarization state. The detected intensity versus rolation then follows the response shown in the bottom of Fig. 2-2 and is described by

$$
I(i, \omega)=\frac{1}{2} I_{0}[1 \pm \sin [2 \Theta(\omega)]]
$$

where $\Theta(\omega)$ is the frequency dependent Faraday rotation intraduced in Chapter 3. The linear dependence of $\theta(\omega)$ on the current, $i$, is established by Eq. (3-4). Maximurn sensitivity to the Faraday rotation is achieved since the slope of the response curve in Eg. (4-1) is largest at zcro current. The choice of sign in Eq. (4-I) depends on whether a $+45^{\circ}$ or $-45^{\circ}$ analyzer selling is used. For the RF currents ased in testing, the bipolar signals oscillate about zero and the magnitude of the ac component is all that is recorded. Then in the absence of any linear birefringence in the coil fibers, the sign of the responsc slope near zero in Eq. (4-J) is not important, and the system output polarizers can be set at either $+45^{\circ}$ or $-45^{\circ}$. In the present measurements, maximum rotations were approximalely $200 \mathrm{Hrad}$ (1ms) making the argument of Eq. (4-1) very small. Under these conditions, the change in the measured light intensity is directly proportional to changes in the induced Faraday rotation, $\Delta \mathrm{I} \circ \Delta \Theta$. Subsequently, the intensity variations follow the signal drive curent, $\Delta \mathrm{I} \propto \Delta \mathrm{i}$.

Spectrum analysis was used to determine the Faraday rotation in the fiber sensor coils through frequency domain measurements of response magnitudes. No attempts were made at measuring phase shifts in the sensor systems or monitoring the time domain response. Instrument sensitivities make such measurements far more difficult than the magnitude determination. Relative response magnitudes were generated for each of the current sensor coils of interest by establishing a low frequency reference. For all of the systems studied, this reference magnitude was recorded at $1 \mathrm{MHz}$ or below. At discrete higher frequencies magnitude changes were measured. A plot of the relative 
frequency response was produced directly from these results. Care was taken during all measurements to keep the RF power transmitted through the cell and the dc laser light level constant. Any significant deviations in these parameters were corrected in the measured magnitudes.

The small Faraday rotation angles produced in the silica optical fibers reguired measuring signals with magnitudes less than $-70 \mathrm{dBm}$. Photodetection was usually limiled by thermal noise at a level near $-120 \mathrm{dBm}$ for a $10 \mathrm{~Hz}$ spectrum analyzer bandwidth. When using the GaAlAs semiconductor diode laser sources, carrier noise often dominated the system; levels were typically 10 to $20 \mathrm{~dB}$ greater than detecior thernal noise. Signal-to-noise ratios $(\mathrm{S} / \mathrm{N})$ at the lowest fregtencies $(-1 \mathrm{MHz})$ were always greater than $30 \mathrm{~dB}$, regardless of the source ased, and occasionally exceeded 40 $\mathrm{dB}$ for some of the fiber sensor systems. At the higher frequencies the S/N dropped as the response diminished. Consequently, significant RF shielding was required to preserve signal integrity at these higher frequencies. Isolation between the RF drive amplifier output (up to $+40 \mathrm{dBm}$ ) and the spectrum analyzer input (noise levels at less than $-110 \mathrm{dBm}$ ) needed to be greater than $150 \mathrm{~dB}$ up to $1 \mathrm{GHz}$ Shielding was provided at all component stages: semirigid coaxial cable for all electrical interconnects; the closed coaxial test cell; multimode fiber isolation of the detected optical intensity; complete shielded enclosures with coaxial feedthroughs for the photodetection system; and shielded, battery-operated sources for supply voltages. RF interference occastonally degraded system performance at the highest frequencies, but did not prohibit the characterization of any current sensor coil response. 


\section{CHAPTER 5 \\ CENTERED AND CIRCULAR FIBER SENSOR COILS}

The most common configuration for a Faraday effect fiber current sensor is a planar, circularly shaped coil with the current-carrying conductor centered in the coil cross section. This geometry is considered baseline for most sensor systems, and experimenters often strive to assemble fiber coils in this configuration if for no other reason than the resultant symmetry. Because of the high degree of symumetry, the mathematical developments needed to describe the high frequency response of the Faraday rotation are greally simplified. Mach of the related theory for the circular, cenlered coil is presented in Ref. 33; it is repeated here to provide a foundation for the circular coils and establish comparative relationships for tater model developments.

\section{Centered Coll Model}

In addition to earlier assumptions given in Chapter 3 , it is now assumed that all fiber tums are circular in cross section, collocated in space and concentric with the conductor axis. Then if the frelds associated with the current are planar, all points on the fiber coil are simultaneously exposed to the same magnetic flux density. Variations in field modulation with time are all that create a frequency dependence in the indnced Faraday rotation. In Eq. (3-8), $\mathrm{r}$ is a constant value $\mathrm{R}$, which is the fiber coil radius, and $\gamma$ is zero at all times since the magnetic field vector direction always coincides with the direction of light propagation in the fiber. Equation (3-8) then reduces to the simple form:

$$
\theta(\omega)=\theta_{0}\left(\frac{1}{\tau}\right) \int_{0}^{\tau} e^{-j \omega t} d t
$$


where $\tau=(n / c) L=2 \pi R N(n / c)$. The solution to Eq. $(5 \cdot 1)$ is

$$
\theta(\omega)=\theta_{0}\left(\frac{1}{\tau}\right) \frac{1-e^{-j \omega \tau}}{j \omega},
$$

which further simplifies to

$$
\Theta(\omega)=\theta_{0}\left[\frac{\sin (\omega \tau / 2)}{(\omega \tau / 2)}\right] e^{-j \omega \tau / 2}
$$

Equation (5-3) is a familiar relationship, describing the response of many systems involving the convolution of two propagating electromagnetic signals [62]. The magnitude of the Faraday response relative to its low frequency value is

$$
\left|\frac{\Theta(\omega)}{\theta_{0}}\right|=\left|\frac{\sin (\omega \tau / 2)}{(\omega \tau / 2)}\right| .
$$

and the phase, $\phi$, is given by

$$
\phi=\arg [\Theta(\omega)]=-\frac{\omega \tau}{2}
$$

Plots of the magnitude and phase described by Egs. (5-4) and (5-5) are shown in Fig. 5-1 as a function of the normalized frequency, $\omega \pi / 2$. Presentation of theoretical responses versus nomalized frequency removes the dependence on coil size. This practice is commonly used throughout this document.

In the most generalized case, Eq. (5-3) also includes a phase factor, e-joto, which accounts for an arbitrary starting point of the light in the ftber coil relative to the zero 


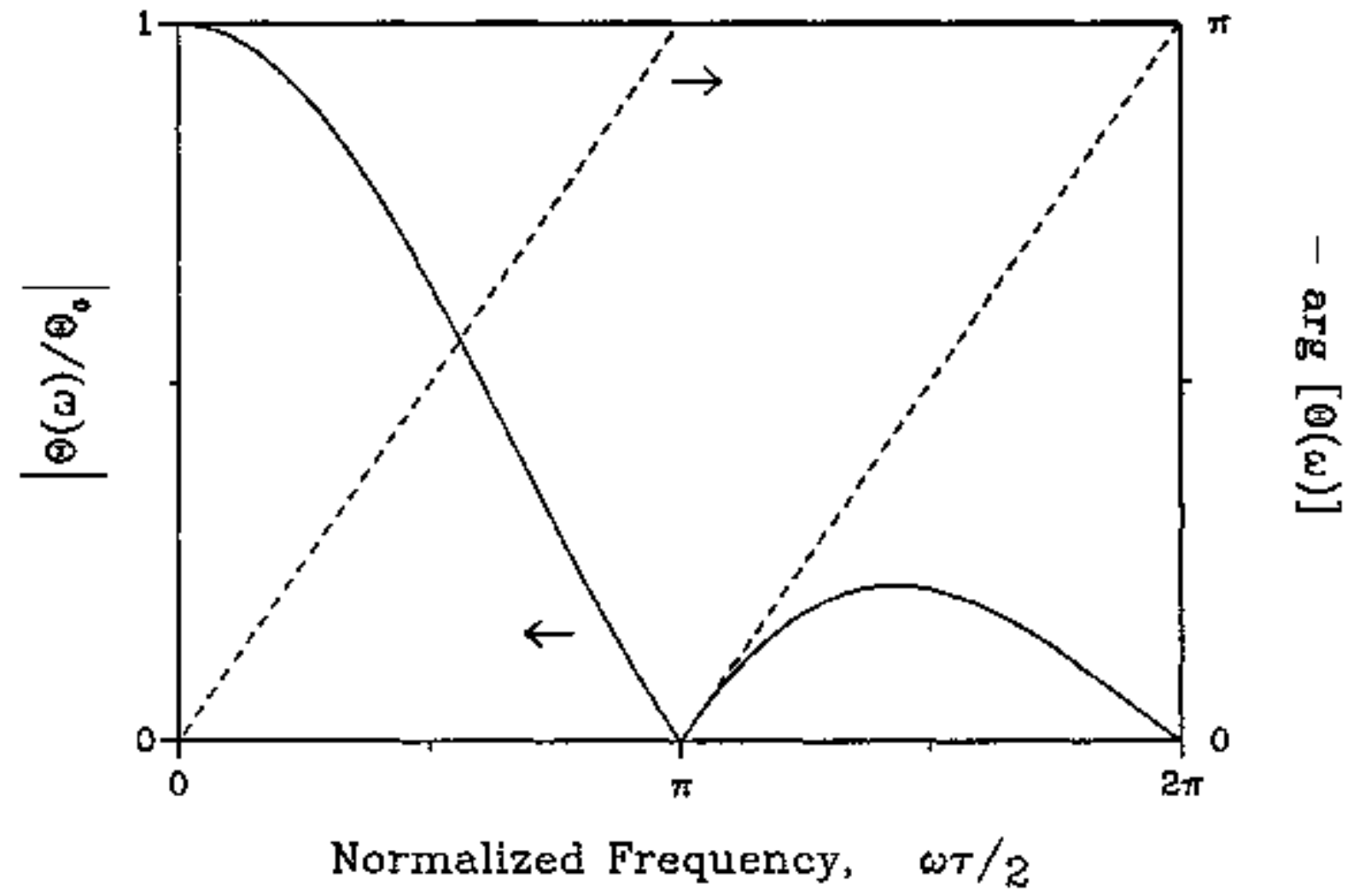

Fig. 5-1. The relative magnitude (solid curve) and phase (dashed curve) of the frequency dependent Faraday sotation for an optical fiber current sensor systern with a centered, circular coil.

phase point of the oscillating magnetic field. The time delay, $\mathrm{t}_{0}$, can be introduced into Eq. (5-1) by modifying the integration limits, to to $\tau+t_{0 p}$ or by changing the integrand to $\left.e^{-j o(t}+t 0\right)$. Using either approach, the resulting phase delay is the same and has no affect on the Faraday rotation magnitude, Eg. (5-4). The response phase becomes $\phi=$ $-(0 / 2)\left(\tau+t_{0}\right)$, but its frequency dependence is not altered from that in Eq. (5-5). Consequently, the arbitrary phase delay has no signiticant affect on Faraday response for the centered, circular coils, and $t_{0}$ is set to zero without any loss of generality.

By combining Eqs. (3-10) and (5-4), the $3 \mathrm{~dB}$ bandwidth, $\Delta \mathrm{B}$, occurs when 


$$
\left|\frac{\sin \left(\omega_{\mathrm{u}} \tau / 2\right)}{\left(\omega_{\mathrm{u}} \tau / 2\right)}\right|=\frac{1}{\sqrt{2}},
$$

from which $\omega_{u} \tau / 2=1.392$ and

$$
\Delta B=\frac{0.696 \mathrm{c}}{\pi^{2} \operatorname{RnN}}
$$

An estimate of the sensor bandwidth was made in Ref. 33 by noting that $\omega_{u} \tau / 2 \approx \sqrt{2}$ from Eq. (5-6). Thus

$$
\Delta B=\frac{c}{\sqrt{2} \pi^{2} \operatorname{RnN}}
$$

The difference in the bandwidths computed using Egs. (5-7) and (5-8) is only 1.6\%. The current sensor high frequency cutoff (first response null) occurs at

$$
\mathrm{f}_{\mathrm{co}}=\frac{1}{\tau}=\frac{\mathrm{c}}{2 \pi \mathrm{RnN}}
$$

Additional response function nulls occur at integer moltiples of $f_{c 0}$.

A sensitivity-bandwidth product was introduced in Ref. 33 and has been used by other researchers [30] to compare the high frequency measurement performance of current sensing systems, both optical fiber and bulk optic. The sensitivity-bandwidth product is formed by combining Eqs. (2-3) and (5-8):

$$
\mathrm{SBP}=\left[\left(\frac{\Theta_{0}}{i}\right) \cdot \Delta B\right]=\frac{\mu_{f} V c}{\sqrt{2} \pi^{2} \mathrm{R} n} .
$$


Equation (5-10) indicates that fiber corrent sensor bandwidth can be increased without a loss in sensitivity by reducing the coil radius. At small radii, however, the effects of bend-induced linear birefringence begin to quench the Faraday sensitivity [50]. Under such conditions, the SBP is a more complex relationship involving $R$. In fact, the response function for fiber coils that exhibit significant linear birefringence will contain other frequency dependent factors in addition to those described by Eq. (5-3). During experimentation with one tightly wound coil, this aromalons behavior was observed. It was quickly dismissed as being caused by linear birefringence; no mathematical description of this perturbed frequency response was attempted.

As mentioned previously, the effects of linear birefringence can be overcome in the fiber sensor by twisting the optical fiber [52] before forming it into a coil. This procedure creates a circular birefringence bias that leads to acceptabie sensor response. Praclical mechanical limilations on fiber twisting, however, are approximately 50 twistsim, which constrains coil radil to greater than $1.5 \mathrm{~cm}$ for current sensor operation in the red or near infrared portions of the spectrum. Smaller coil radii are possible by using fiber annealing to remove bend-induced birefringence and the intrinsic stresses doe to manufacturing [21]. Coils of 10 to 20 turns with $2.5 \mathrm{~mm}$ radii [63] and 100 to 200 turns with $5 \mathrm{~mm}$ radii [54] have been manufactured. With fiber sensors having these small radii, significant improvements in the sensitivity-bandwidth product are possible. Thus, high frequency measurements on an annealed coil sensor are included in this study to demonstrate these improvements.

\section{Measured Response Functions}

High frequency responses were measured on several optical fiber current sensor coils to demonstrate the ulility of Faraday effect sensors at extremely high frequencies and to verify the theoretical predictions. Fiber test coils were fabricated in several configurations; these are summarized in Table 5-1. Mean coil diameters ranged from 
Table 5-1.

Summary of High Frequency Response Measurements

on Centered, Circular Optical Fiber Current Sensor Coils.

\begin{tabular}{|c|c|c|c|c|c|c|c|c|}
\hline \multirow[t]{2}{*}{$\begin{array}{c}\text { Coil } \\
\#\end{array}$} & \multirow{2}{*}{$\begin{array}{c}\text { Meso } \\
\text { Coil } \\
\text { Dímeter } \\
\text { (cm) }\end{array}$} & \multirow[t]{2}{*}{$\begin{array}{c}\text { Number } \\
\text { of } \\
\text { Tums }\end{array}$} & \multirow[t]{2}{*}{$\begin{array}{c}\text { Fibert } \\
\text { Coil } \\
\text { Construtetion }\end{array}$} & \multicolumn{2}{|c|}{$\begin{array}{c}3 \mathrm{~dB} \\
\text { Bandwidth, } \\
\Delta \mathrm{B}(\mathrm{MHz})\end{array}$} & \multicolumn{2}{|c|}{$\begin{array}{c}\text { Outoff } \\
\text { Frequency, } \\
f_{\text {co }}(M \mathrm{~Hz})\end{array}$} & \multirow{2}{*}{$\begin{array}{l}\text { Highest } \\
\text { Messurement } \\
\text { Frequency } \\
\text { (MHz) }\end{array}$} \\
\hline & & & & Measured & Theory & Measured & Theory & \\
\hline 1 & 30.7 & 100 & $\begin{array}{c}\text { Twisted, 15/m } \\
\text { Rejacketed }\end{array}$ & 2.7 & 2.7 & 6.1 & 6.1 & 15 \\
\hline 2 & 9.5 & 42 & Twisled, $10 / \mathrm{m}$ & 7.1 & 7.3 & 16.1 & 16.4 & 50 \\
\hline 3 & 6.0 & 20 & $\begin{array}{c}\text { Twisled, 40fm } \\
\text { Rojacketed }\end{array}$ & 24.5 & 24.2 & 55.4 & 54.5 & 110 \\
\hline 4 & 6.0 & 10 & $\begin{array}{c}\text { Twisted, 40/m } \\
\text { Rejacketed }\end{array}$ & 45.4 & 48.3 & 102.5 & 109.0 & 215 \\
\hline 5 & 8.95 & 15 & $\begin{array}{c}\text { Twisted, } 40 / \mathrm{m} \\
\text { Rejacketed }\end{array}$ & 20.7 & 21.6 & 46.8 & 48.7 & 100 \\
\hline 6 & 0.64 & 20 & Annealed & 229.1 & 226.4 & 517.1 & 511.0 & 1000 \\
\hline 7 & 9.6 & 10 & $\begin{array}{c}\text { Twisted, 40/m } \\
\text { Rejecketed }\end{array}$ & 29.5 & 30.2 & 66.7 & 68.1 & 800 \\
\hline 8 & 2.95 & 21 & Twisted, $20 / \mathrm{m}$ & 46.7 & 46.8 & 105.4 & 105.6 & 215 \\
\hline
\end{tabular}


approximately $6.4 \mathrm{~mm}$ to $10.7 \mathrm{~cm}$ and the number of coil turns varied between 10 and 100. The smallest coil, \#6, was annealed during fabrication while all the other coils used twisted fiber.

(The annealed coil was prepared by the Optical Electronic Metrology Group at the National Institute of Standards and Technology (NIST). NIST personnel also conlributed significantly to the fabrication and characterization of the coaxial transmission cell used to hold the coil and the experimental measurement of the sensor response.)

A summary of the high frequency response parameters for each of the different sensors is also presented in Table \$-1. Both measured and theoretically predicted values are given for bandwidth and cutoff frequency. Equations (5-7) and (5-9) were used for the predictions. The $3 \mathrm{~dB}$ bandwidths for the coils in Table 5-1 ranged from 2.7 to 229 $\mathrm{MH} /$. High frequency measurements were made on all coils 10 well beyond $\mathrm{f}_{\mathrm{co}}$; the largesl tesl fregiency was $1 \mathrm{GHz}$. For some of the configurations several high frequency lobes of the response magnitude (greater than two) were investigated. All of the measured response curves followed the functional form of the magnitude predicted by Eq. (5-4) and plotted in Fig. 5-1.

The frequency response of coil \#4 listed in Table 5-1 is shown in Fig. 5-2. The dashed curve is generated from $\mathrm{Eq}$. (5-4) using the eslimated parameters for the fiber ( $n=1.46$ at $\lambda=633 \mathrm{~mm}$ ) and coil only. Although the curve shape is generally correct, it represents a poos fit to the experimental values. Discrepancies are due somewhat to the uncertainty in determining coil geometry and the optical fiber refractive index but mostly to neglect of the fiber coil leads in the response computation. When the coil was inserted into the coaxial test cell, the input and output fiber leads were arranged so that each lead exited the coil in opposite directions. This effectively adds an extra half turn to the value of $\mathbf{N}$. (This configuration is not a requirement for current sensor coils and some of the tesled coils did have a different lead configuration.) The added fiber length then 
contributes to a reduced bandwidth since the passing magnetic wave also produces a Faraday rotation in the leads.

An extended model that addresses fiber lead effects is presented in Appendix B. An exact mathematical description is derived for the Faraday rotation in two possible lead configurations: fiber entering and exiting from the same side, and the configuration used for coil $\# 4$, leads exiting in opposite directions. When the lead effects are accounted for in the response of the coil, the solid curve in Fig. 5-2 is produced. This predicted response agiees well with the measured data points, especially at the nulls in the curve. Not surprising is the fact that the solid curve in Fig. 5-2 can be duplicated almost precisely by using $\mathrm{N}=10.5$ as a coll parameter in the magnitude prediction from

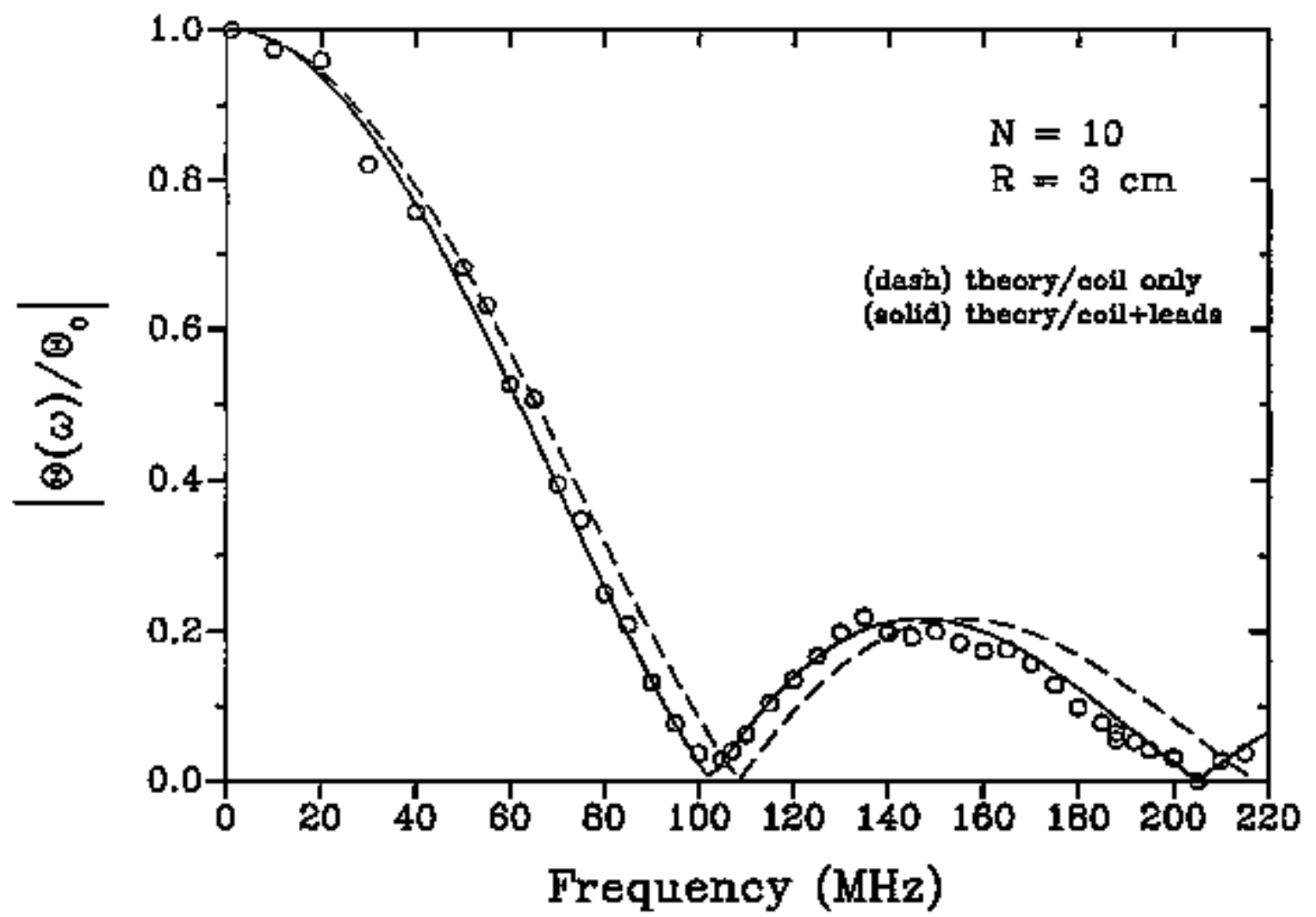

Fig. 5-2. Measured and theoretical Faraday rotation relative response magnitudes versus frequency for a $10 \mathrm{tum}, 6 \mathrm{~cm}$ diameter cument sensor coil. 
Eq. (5-4).

Figure 5-3 is a plot of the relative magnitude of the high frequency Faraday response for the annealed fiber coil sensor (coil \#6 in Table 5-1). The sensor bandwidth is estinated to be $229 \mathrm{MHz}$ and the cutoff frequency $517 \mathrm{MHz}$. The dashed curve in $\mathrm{Fig}$. $5-3$ is a best fit to the measured values using the function given by Eq. (5-4) and varying the argument, $\omega t / 2=2 \pi^{2} \mathrm{RN}(\mathrm{n} / \mathrm{c})$. This fitied curve is quite accurate and thus can be used to enhance the evaluation of the sensor response. Since relative measurement errors are typically $\pm 1 \mathrm{~dB}$, exact determination of response parameters is difficult using only the measured values. For example, the $3 \mathrm{~dB}$ bandwidth is extracled from the experimental

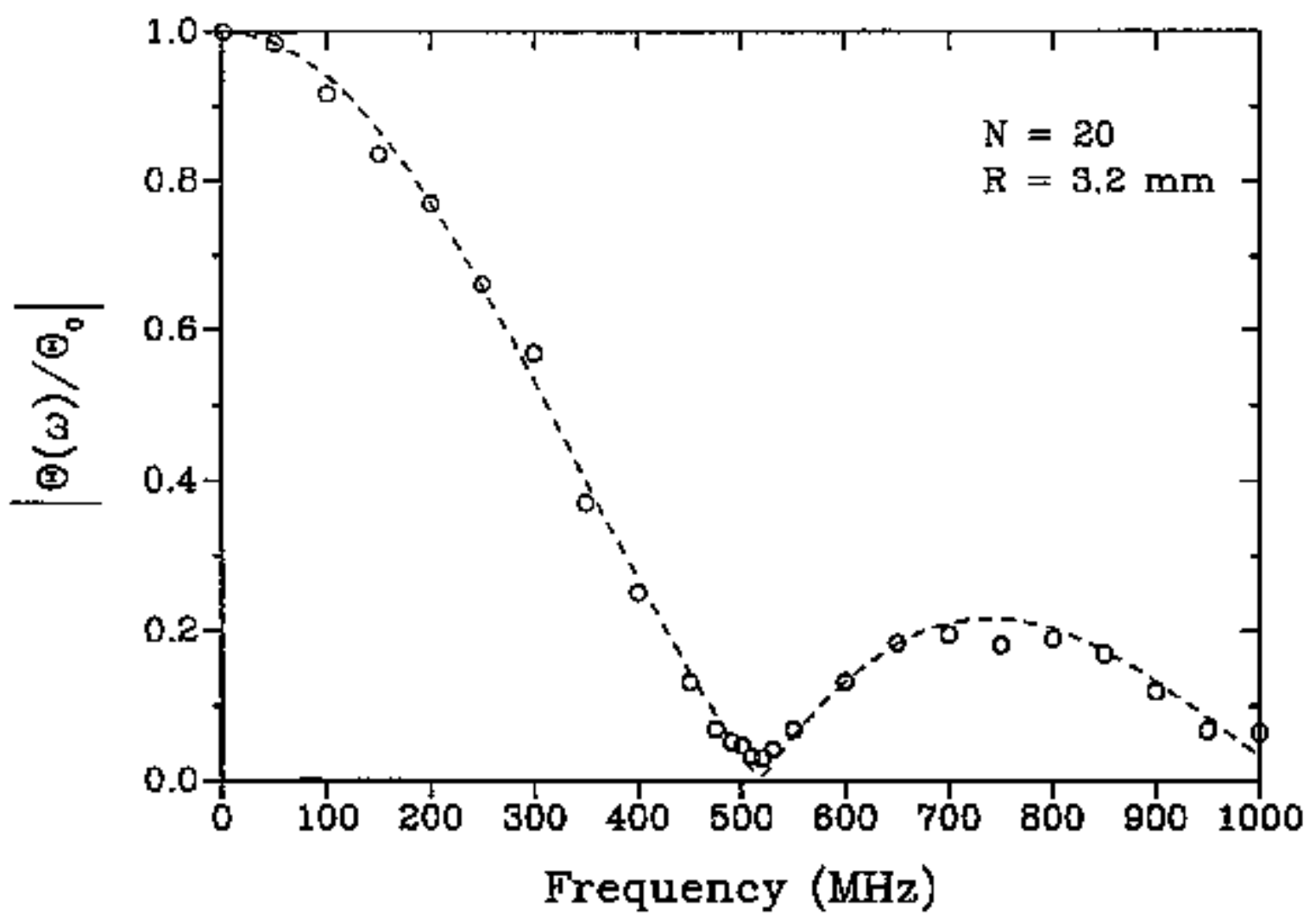

Fig. 5-3. The measured Faraday rotation relative magnitude versus frequency for a 20 turn, $6.4 \mathrm{~mm}$ diameter annealed fiber current sensor coil. The dashed curve is a best fit functional response to the experimental data. 
data by first performing a functional curve fit, taking care to properly match the response nulls since absolute measorement error is small in these frequency regions, and then interpolating the frequency at a relative magnitude of 0.707 . All of the measured bandwidths for sensors presented in Table 5-1 were determined using this procedure.

Fits to the experimental response data are also useful in estimating other fiber and coil parameters. The dashed curve in Fig. 5-3 was used to estimate the mean diameter of the annealed fiber sensor coil. In manufacturing the coil, an annealing fixtore with a coil channel $5 \mathrm{~mm}$ to $8 \mathrm{~mm}$ in diameter is used to confine the fiber. The resulting coil has turns of slightiy varying diameter. From the results presented in Fig. 5-3, a light transit time is estimated for the entire coil which then corresponds to a mean coil diameter of approximately $6.4 \mathrm{~mm}$.

The annealed fiber coil represents a first attempt at producing a current sensor with a very wide bandwidth. Its testing is also a first attempt al characterizing the response of a fiber current sensor into the microwave region $(>500 \mathrm{MHz}$ ). The estimated $3 \mathrm{~dB}$ bandwidth of $229 \mathrm{MHz}$ is almost a factor of five greater than that for any of the other coils characterized in this study. Since the measured Faraday rotations were approximately of the sane magnitude for the annealed coil as for all the other test coils, there appears to be no degradation in sensor performance due to fiber annealing. Hence, the annealing process only serves to enbance the capabilities for high frequency current sensing by extending the sensitivity-bandwidth product. The estimated SBP for this sensor is $1.34 \times 10^{4} \mathrm{~Hz}-\mathrm{rad} / \mathrm{A}$ or $0.77 \mathrm{MHz}-\%$ A.

The signal and noise spectrum of the annealed fiber current measurement test system is shown in Fig. 5-4. Maximum signals at the low freguencies correspond to measured rms rotations of approximately $41.4 \mu \mathrm{rad}$. Diode-laser carrier noise (closed circles in Fig. 5-4) is the liniting factor in sensor performance; it increases in magnitude about $10 \mathrm{~dB}$ as the freguency increases from its lower values to $1 \mathrm{GHz}$. Over the sensor $3 \mathrm{~dB}$ bandwidth, a $\mathrm{S} N \approx 32 \mathrm{~dB}$ is maintained for a spectrom analyzer bandwidth of 10 


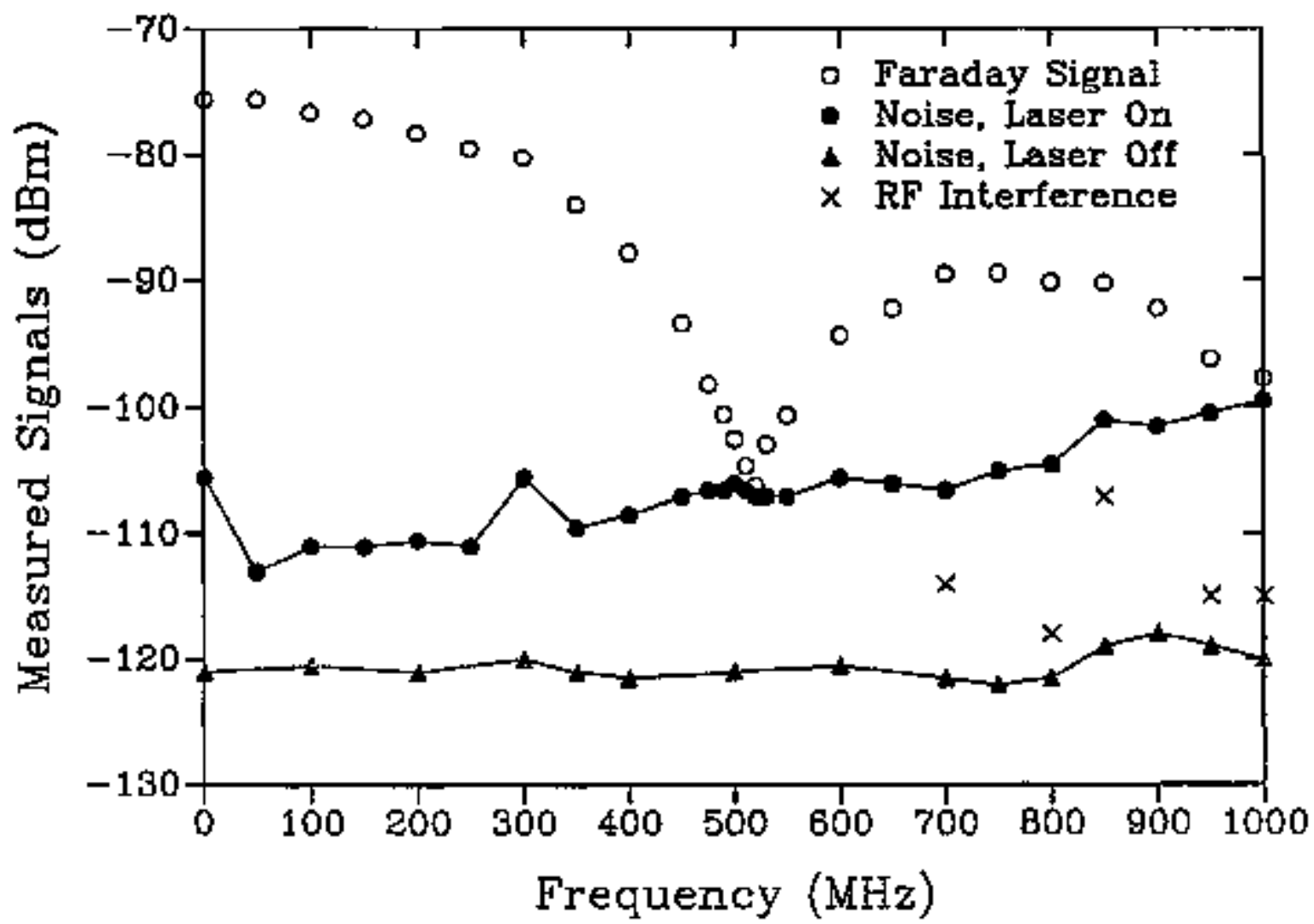

Fig. 5-4. The measured signal and noise responses versus freguency for the annealed fiber current sensing system.

Hz. Based on experimental parameters and measured noise characteristics, the projected noise equivalent ims current (NEI) sensitivity for this annealed coil sensor is $3.6 \mathrm{~mA} / \sqrt{\mathrm{Hz}}$, or if the number of fiber turns in the coil is removed, $71.5 \mathrm{~mA} / \sqrt{\mathrm{Hz}} /$ turn. Over the full sensor $3 \mathrm{~dB}$ bandwidth, the minimum detectable ms current would then be $54 \mathrm{~A}$.

As a comparison, the published NEI sensitivity for a $1 \mathrm{~cm}$ diameter, 188 tum annealed-cojl sensor system is $\sim 100 \mu \mathrm{A} / \sqrt{\mathrm{Hz}}$ or $18.8 \mathrm{~mA} / \sqrt{\mathrm{Hz}} /$ torn [54]. This noise figure is a factor of four better than that deternined for the wideband annealed coil system studied here. However, the $3 \mathrm{~dB}$ bandwidth for the sensor in Ref. 54 is only 15.5 $\mathrm{MHz}$, almost a factor of 15 smatler. The differences in NEI performance can be rectified 
by comparing the detection systems used in each study. In Ref. 54 , the sensor sensitivity is based on a shot-noise-limited detection of two, complimentary polarization-state signals (se Eq. (2-10) and Fig. 2-5). Wideband sensing systems as used in the present measurements require $50 \Omega$ or low impedance circuitry to be compatible with RF instrumentation. Thus, detector noise levels are dominated by thermal properties and not shot noise. Thermal noise levels in these systems will typically be $10 \mathrm{~dB}$ or greater than detector shot noise. The detector thermal noise measurements for the annealed coil used here are given in Fig. 5-4 as the closed triangles. If this system was limited by thermal noise instead of laser canjer noise (which is frequency dependent), the NEI sensitivity would rival that of the systems presented in Ref. 54. In fact, the daal detector system used in Ref. 54 allows for removal of laser noise common in the two channels.

In most of the measurements on the sensor systems summatized in Table 5-1, a He-Ne laser was used as the source. Performances were then limited by detector thermat noise and not jaser cartier noise as seen in Fig. 5-4. This leads to larger signal-to-noise ratios and smaller broadband NEIs. The largest measured S/N vatues were for coil \#8. Over its $3 \mathrm{~dB}$ bandwidth of $46.7 \mathrm{MHz}, \mathrm{S} / \mathrm{N}$ is greater than $40 \mathrm{~dB}$, and the system ms NEI is $1.4 \mathrm{~mA} / \sqrt{\mathrm{Hz}}$ or $30 \mathrm{~mA} / \sqrt{\mathrm{Hz}} / \mathrm{tum}$. This sensor has a relatively narrow bandwidth compared to that of the annealed coil sensor used in this study, and thus the minimum detectable rms current at full bandwidth is significantly smaller, 3.1 A. It is obvious that complex tradeoffs exist between sensitivity and bandwidth in these high frequency systems. An estimated theoretical limit for the ultimate noise performance in a fiber current sensor system utilizing the dual shot-noise-linited detectors was briefly presented in Chapter 2 and is given as $1 \mathrm{~mA} / \sqrt{\mathrm{Hz}} /$ tum $[45,59]$. It must be remembered that this estimate is based on low frequency signals and assembling such a system for wide bandwidth current monitoring may not be possible.

The maximum measurement frequency for each of the fiber current sensor coils is listed in the last column of Table 5-1. Characterizations were performed at frequencies 
that extend well beyond the cutoff frequency. In general, the measured magnitudes for the second lobes of the response functions show excellent agreement with the theoretical predictions. This can be seen in the response plots of Figs. 5-2 and 5-3. For some of the coil systems, several of the higher frequency response lobes were measured, and magnitudes always agreed with the predictions. (One such response curve, for coil $\# 7$, is shown in Fig. 6-6a and covers 12 frequency lobes.) The purpose of measuring corrent sensor frequency response beyond $f_{c o}$ is to produce a full understanding of the relative Faraday rotation magnitude as described in Eq. (5-3). By performing fits to the measured magnitude data, an accurate value for $\omega t / 2$ can be extracted for a given sensor coil. This then gives additional informatton about the sensor phase frequency response, Eq. (5-5), since the phase angle is much more difficult to measure experimentally. It is postulated that with enough knowledge of the high frequency response magnitude and phase, these Faraday effect current sensor coils could be used as wideband monitoring devices to frequencies above their $3 \mathrm{~dB}$ bandwidth. Near $\mathrm{f}_{\mathrm{co}}$, however, the sensor response would eventually be limited by system $\$ / N$. 


\section{CHAPTER 6}

\section{NONCENTERED CURRENT SENSOR COILS}

Fiber current sensor coils cannot always be properly aligned to have the coil and conductor axes coincident. In some applications, it may not be possible or even desirable. The high frequency Faraday response has been investigated for circular current sensor coils whose axis has a linear radial displacement with respect to the conductor. A model bas been developed that predicts the frequency dependent Faraday rotation for such sensors. Experiments have verified the model for specific cases. In general, the radial displacement can assume any value, even those where the conductor lies outside of the coil cross section. The entry point of the light into the fiber coil relative to the conductor also plays an important role in coils with one or a few turns. Both theory and experiment indicate high frequency resonant responses for these noncentered coils. The magnitude of these resonances is dependent on several properties of the coil-conductor system.

\section{Noncentered Coll Model}

A noncentered current sensor coil is modeled using the geometry displayed in Fig. 6-1. The coil and field parameters are identical to those identified previously for the centered coil model, except here, the conductor axis is radially displaced from the coil axis by a distance $X_{1}$. This displacement means that light propagating around the fiber loop will experience a spatially-dependent magnitude variation in the passing magnetic field. Light enters and exits the coil at a single point designated by the angle, $\alpha_{n}$, from the conductor position. Coil leads are neglected in this model. The current conductor is again treated as a filament of moving charge with point cross section. This allows for modeling of the specific configuration $\mathrm{X}_{1}=\mathrm{R}$ or where the conductor is at the fiber.

From Fig. 6-1, the radial distance, $r$, between the conductor and the propagating light element, di, on the fiber coil is given by the law of cosines: 


$$
\mathbf{r}^{2}=\mathrm{R}^{2}+\mathrm{X}_{1}^{2}-2 \mathrm{RX} \mathrm{X}_{1} \cos \left(\alpha+\alpha_{0}\right)
$$

The angle $\gamma$ between the magnetic flux density and the light element is atso determined from the law of cosines. The relationship is rearranged to give

$$
\cos \gamma=\frac{\mathrm{R}^{2}+\mathrm{r}^{2}-\mathrm{X}_{1}^{2}}{2 \mathrm{Rr}} .
$$

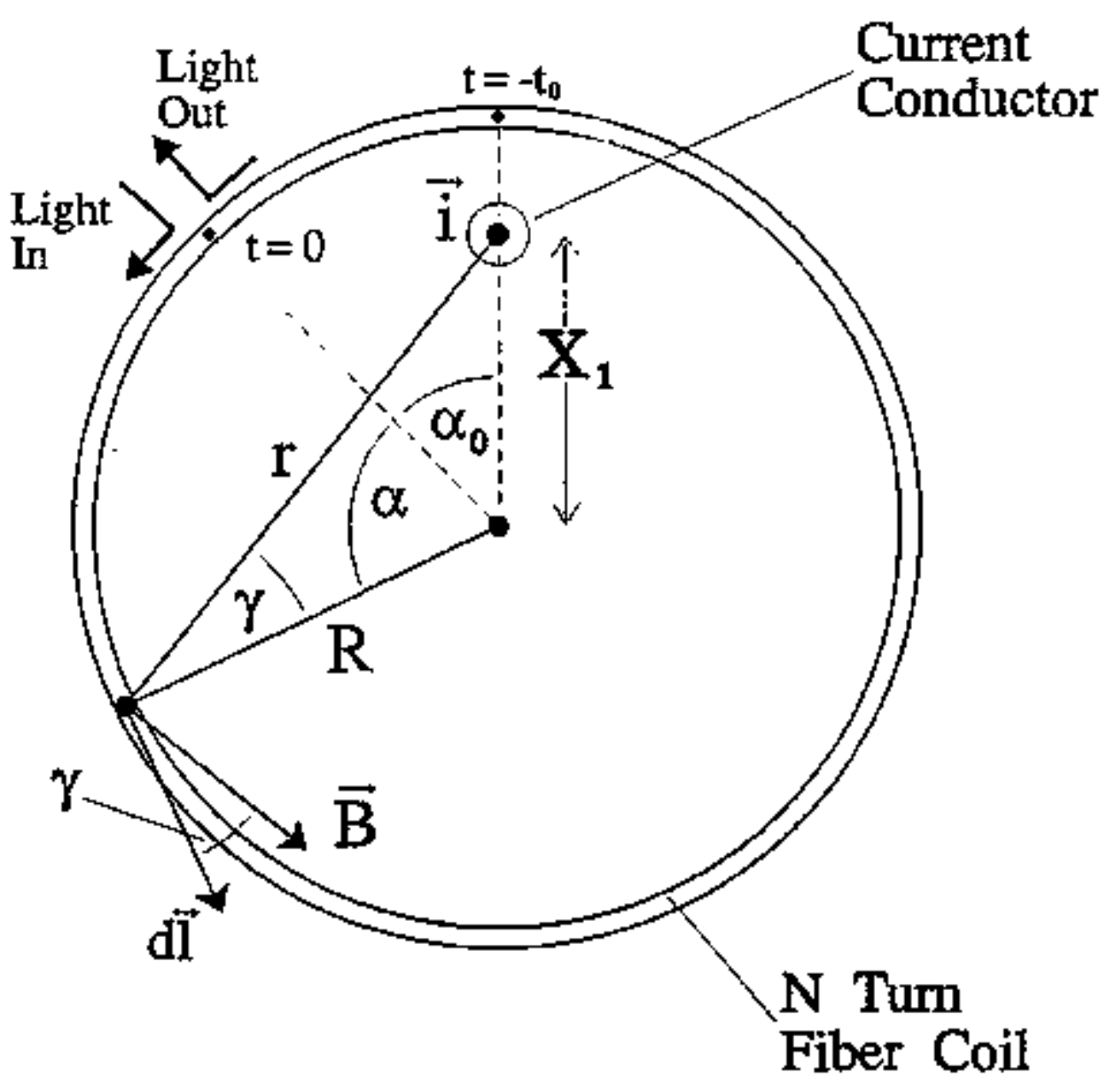

Fig. 6-1. A geometrical model of a fiber current sensor coil with the conductor displaced from the coil axis. 
Combining Egs. (6-1) and (6-2) yields the following expression for substitution into the integrand of Eq. (3-8):

$$
\frac{\cos \gamma}{r}=\frac{1}{R}\left[\frac{1-\left(\mathrm{X}_{1} / \mathrm{R}\right) \cos \left(\alpha+\alpha_{0}\right)}{1+\left(\mathrm{X}_{1 / \mathrm{R}}\right)^{2}-2\left(\mathrm{X}_{1 / \mathrm{R}}\right) \cos \left(\alpha+\alpha_{0}\right)}\right] .
$$

The required time dependence of $\gamma$ and $\mathrm{r}$ in Eq. (6-3) is provided by the rotating angle, $\alpha$. As the light in the fiber propagates, this angle changes as

$$
\alpha(t)=\frac{c}{n R} t
$$

The reference starting angle is then given by $\alpha_{0}=(\mathrm{c} / \mathrm{nR}) \mathrm{t}_{0}$.

Equation (6-3) can be transformed into an infinite series by using simple long division:

$$
\frac{\cos \gamma}{r}=\frac{1}{R} \sum_{m=0}^{\infty}\left(\frac{X_{1}}{R}\right)^{m} \cos \left[m\left(\alpha+\alpha_{0}\right)\right]
$$

Since the cosine function oscillates between 1 and -1 , this series converges only when $X_{1}<R$. Substituting Eq. (6-5) into Eq. (3-8) provides the integral expression for the frequency dependent Faraday rotation,

$$
\Theta(\omega)=\Theta_{0} \frac{1}{\tau} \sum_{\mathbb{m}=0}^{\infty}\left(\frac{X_{1}}{R}\right)^{m} \int_{0}^{\tau} \cos \left[m \frac{c}{n R}\left(t+t_{0}\right)\right] e^{-j \omega t} d t
$$

where $\tau=(\mathrm{n} / \mathrm{c}) \mathrm{L}=(\mathrm{n} / \mathrm{c}) 2 \pi \mathrm{NR}$ is used in the factor preceding the summation. In 
Eq. (6-6) the summation and integration have been interchanged under the assumption that the series is absolutely convergent. The integration proceeds by rewriting the cosine function in its exponential form,

$$
\frac{1}{2}\left[e^{j \frac{m c}{n R}\left(t+t_{0}\right)}+e^{-j \frac{m c}{\mathrm{nR}}\left(t+t_{0}\right)}\right]
$$

and rearranging 10 get

$\theta(\omega)=\theta_{0} \frac{1}{\tau} \sum_{m=0}^{\infty}\left(\frac{X_{1}}{R}\right)^{m}\left(\frac{1}{2}\right)\left[e^{j \frac{m c}{n R} l_{0}} \int_{0}^{\tau} e^{-j\left(\frac{m c}{m R}-\omega\right) t} d t+e^{-j \frac{m c}{a R} l_{0}} \int_{0}^{\tau} e^{-j\left(\frac{m c}{n R}+\omega\right) t} d t\right]$.

Continuing with the integration leads to

$$
\begin{aligned}
\Theta(\omega)= & \theta_{0} e^{-j \omega \tau / 2}\left(\frac{1}{2}\right) \sum_{m=0}^{\infty}\left(\frac{X_{1}}{R}\right)^{m} \\
& {\left[\frac{\sin \left[\left(\omega-\frac{m c}{n R}\right) \frac{\tau}{2}\right]}{\left[\left(\omega-\frac{m c}{n R}\right) \frac{\tau}{2}\right]} e^{j \frac{m c}{n R}\left(t_{0}+\tau / 2\right)}+\frac{\sin \left[\left(\omega+\frac{m c}{n R}\right) \frac{\tau}{2}\right]}{\left[\left(\omega+\frac{m c}{n R}\right) \frac{\tau}{2}\right]} e^{\left.-j \frac{m c}{n R}\left(t_{0}+t / 2\right)\right] .}\right.}
\end{aligned}
$$

The expression for the light transit time, $\tau$, in the $\mathrm{N}$-turn circular coil can be rewritten in the form

$$
\frac{m c}{2 n R} \tau=m N \pi
$$

Eqguation (6-9) is substituted throughout Eq. (6-8). The phase factors for each term in the brackets of Eq. (6-8) further reduce to 


$$
e^{ \pm j m N \pi\left(2 t_{0} / \tau+1\right)}=(-1)^{m N\left(2 t_{0} / \tau+1\right)},
$$

since $\mathrm{e}^{-\mathrm{j} \pi}=\mathrm{e}^{\mathrm{j} \pi}=-1$. In this model both $\mathrm{m}$ and $\mathrm{N}$ are always integers. The time delay, $t_{0}$, is unique only for values between zero and the transit time of light through a single loop of the coil, $\tau_{1}=\tau / \mathrm{N}$. It is appropriate then to redefine $t_{0}$ as a fraction of $\tau_{1}$ by

$$
\mathrm{t}_{0}=\xi \tau_{1}=\xi \frac{\tau}{\mathrm{N}}
$$

where $\xi$ is the fractional multiplier having values $0 \leq \xi<1$. A simplified expression for the noncentered sensor coil response is then

$$
\Theta(\omega)=\Theta_{0} \mathrm{e}^{-j \omega t / 2}\left(\frac{1}{2}\right) \sum_{m=0}^{\infty}(-1)^{m(N+2 \xi)}\left(\frac{X_{1}}{R}\right)^{m}\left[\frac{\sin (\omega t / 2-m N \pi)}{(\omega t / 2-m N \pi)}+\frac{\sin (\omega t / 2+m N \pi)}{(\omega t / 2+m N \pi)}\right] .
$$

Equation (6-12) can be further simplified by combining the two terms in the bracket. First, a common denominator is found for this factor to yield

$$
\left[\frac{(\omega t / 2)[\sin (\omega t / 2 \cdot m N \pi)+\sin (\omega \pi / 2+m N \pi)]+(m N \pi)[\sin (\omega t / 2-m N \pi)+\sin (\omega \pi / 2+m N \pi)]}{(\omega \pi / 2)^{2}-(m N \pi)^{2}}\right] .
$$

The trigonometric identities for the sum and differeace of two angles are then applied to reduce this factor to

$$
\left[\frac{(\omega \pi / 2) 2 \sin (\omega \tau / 2) \cos (\mathrm{mN} \pi)}{(\omega \pi / 2)^{2}-(\mathrm{mN} \pi)^{2}}\right]=(-1)^{\mathrm{mN}} \frac{2 \sin (\omega \pi / 2)}{(\omega \pi / 2)}\left[1-\left(\frac{\mathrm{mN} \pi}{\omega \tau / 2}\right)^{2}\right]^{-1},
$$


where $\cos (\mathrm{mN} \pi)$ has become $(-1)^{\mathrm{mN}}$. Substitution of Eq. (6-13) into Eq. (6-12) leads to a final expression for the Faraday rotation in a noncentered sensor coil:

$$
\Theta(\omega)=\Theta_{0}\left[\frac{\sin (\omega \tau / 2)}{(\omega \pi / 2)}\right] e^{-j \omega \tau / 2} \sum_{m=0}^{\infty}(-1)^{2 m \xi}\left(\frac{X_{1}}{R}\right)^{m}\left[1-\left(\frac{m N \pi}{\omega t / 2}\right)^{2}\right]^{-1}
$$

Equation (6-14) can be compared to Eq. (5-3) for the centered coil; obvious similarities exist. If $X_{1}$ is set to zero in Eq. (6-14), only a single term in the series will exist, that being $m=0$. Then the frequency dependent rotation reduces to Eq. (5-3) as required.

The low frequency response for the noncentered coil sensor can also be evaluated. This is more easily accomplished using Eq. (6-12) instead of Eq. (6-14). If $\omega=0$. Eq. (6-12) becomes

$$
\Theta(0)=\Theta_{0}\left(\frac{1}{2}\right) \sum_{m=0}^{\infty}(-1)^{m(N+2 \xi)}\left(\frac{X_{1}}{R}\right)^{m} \frac{2 \sin (m N \pi)}{m N \pi}
$$

But $\sin (m N \pi) /(m N \pi)$ is non-zero only for $m=0$. Then for any value of $X_{r} / R, \Theta(0)=\Theta_{0}$ as expected.

The response for the noncentered, circular coil given by Eq. (6-14) shows a dependence on the entry point for light propagation in the coil, the relative displacement of the coil axis, and the number of coil terns. The starting point for light in the fiber coil

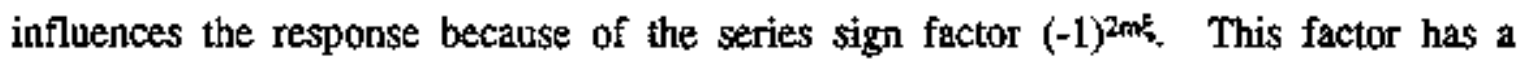
diminished effect when $N \gg \xi$, or when the number of turns becomes large. Resonances will occur in the frequency response when $\omega \tau / 2$ is equal to inleger multiples of $\mathrm{N} \pi(\omega \mathrm{c} / 2$ $=m^{\prime} N \pi$, for $\left.m^{\prime}=1,2,3, \ldots\right)$. At each resonant frequency, $\omega_{k}$, the series in Eq. (6-14) has only one term where $\mathrm{m}^{\prime}=\mathrm{m} \neq 0$. (Note that $\mathrm{m}^{\prime}=0$ produces the low frequency response.) The response given by Eq. (6-14) then becomes 


$$
\Theta\left(\omega_{R}\right)=\theta_{0}\left(\frac{1}{2}\right)(-1)^{2 m^{\prime} \xi}\left(\frac{X_{1}}{R}\right)^{n^{\prime}}
$$

which has a relative magnitude of

$$
\left|\frac{\Theta\left(\omega_{R}\right)}{\Theta_{0}}\right|=\frac{1}{2}\left(\frac{X_{1}}{R}\right)^{m^{\prime}}
$$

With $X_{1}<R$, each successively higher resonance will have a diminishing magnitude. The maximum relalive response will be $1 / 2$ in the limiting case.

\section{Model Predictions}

Equation (6-14) is evaluated for several different current sensor configurations to illusfrate the effecis of a noncentered coil on the high frequency Faraday response. Of particular interest are coils consisting of one furn for which conductor displacement effects are most pronounced. In Fig. 6-2 the relative magnitude of the Faraday rotation is ploted as a function of the normalized frequency, $\omega t / 2$, for single turn sensor coils. The displayed curves result from varying the relative displacement of the conductor, $X_{1} / R$, for two different entry points of light into the coil, $\xi=0.5 \mathrm{in} \mathrm{Fig.} 6-2 \mathrm{a}$ and $\xi=0 \mathrm{in} \mathrm{Fig.}$ 6-2b. These light entry points correspond to the respective angular positions $\alpha_{0}=\pi$ and $\alpha_{0}=0$ in Fig. 6-1.

In Fig. 6-2, curves are plotted where the conductor has a displacenent of $X_{1}=R$. This particular configuration is not physically realizable since the conductor and the fiber cannot occupy the sane location in space. Also, when $X_{1}=R$, the series convergence criterion established for Eq. (6-5) is violated and a singularity is produced in Eq. (6-3). However, finite solutions to Eq. (6-14) do exist when $X_{1}=R$, Eq. (6-16) being evidence of such. Predictions are consistent with others in the noncentered coil model and represent the limiting response as $X_{i}$ approaches $R$. Under these assumptions, the 

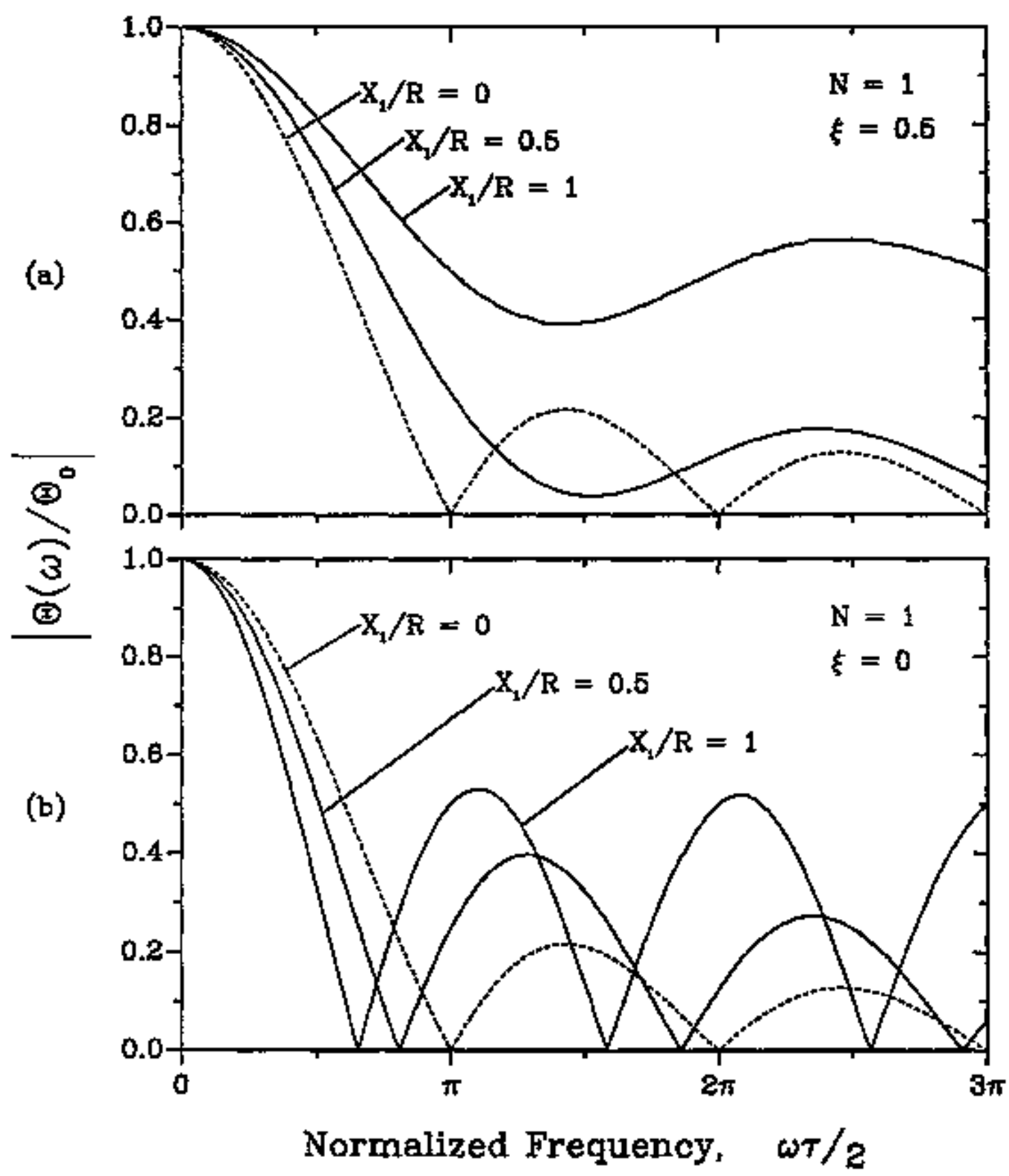

Fig. 6-2. The frequency dependent Faraday rotation response magnitude of a single turn, noncentered current sensor coil. The effects of relative displacement $\left(X_{1} / R\right)$ between the conductor and the coil axis are illustrated for (a) light entry into the coil farthest from the displaced conductor, $\xi=0.5$, and (b) light entry into the coil nearest to the conductor, $\xi=0$. 
theoretical high frequency response at the $\mathrm{X}_{1}=\mathrm{R}$ limit are included in this study as a means of illustrating extreme effects.

For both plots of Fig. 6-2, the $3 \mathrm{~dB}$ bandwidth is changed significantly as the coil and conductor axes become less concentric. In Fig. 6-2a the light enters and exits the single fiber loop at a point farthest from the conductor, $\xi=0.5$, and $\Delta \mathrm{B}$ increases with increasing $X_{J} / R$. Also, the nulls in the response curve disappear, and the higher frequency response lobes change shape, becoming less pronounced. Under these conditions the Faraday rotation does not have a defined cutoff frequency. In contrast, in Fig. 6-2b the light enters and exits the sensor coil nearest to the conductor, $\xi=0$, and $\Delta B$ decreases with increasing $\mathrm{X}_{\mathrm{l}} / \mathrm{R}$. The value of $\mathrm{f}_{\text {co }}$ decreases proportionally with $\Delta \mathrm{B}$, while the response magnitudes in the high frequency lobes increase with $\mathrm{X}_{\mathrm{l}} / \mathrm{R}$. Large response magnitudes appear at frequencies where previously these were nulls. In the limiting cases where $X_{1}=R, \Delta B$ has decreased to approximately 0.7 times that of the centered coil sensor when $\xi=0$ (Fig. 6-2b) and has increased to approximately 1.5 times that of the centered coil when $\xi=0.5$ (Fig. 6-2a).

The dependence of the single tum coil response on $\xi$ can be explained gualitativejy. When the conductor is displaced in the coil cross section, the magnetic field strength is increased at those points of light in the fiber closest to the condactor; the Faraday rotation becomes concentrated in a shorter length of fiber. If the position of the conductor within the coil cross section is away from the light entry point, only one light pass is made through the enhanced region. The decreased effective interaction length naturalty leads to an increased response bandwidth. On the other hand, if the conductor is now positioned toward the light entry point in the coil, the light must twice pass close to the conductor as it propagates around the single loop of fiber. Two regions of strong Faraday interaction are created, and the response is largest when these resonate with the field modulation. This resonance effect is best observed at $\omega \pi / 2=\pi$ in Fig. $6-2 b$ for the curve with $X_{1} / R=1$. Because of the phasing of the magnetic field with the propagating 
light in the coil, an antiresonance must also occur between each of the resonance peaks. The first antiresonance in the Faraday response occurs somewhere between the frequencies $\omega \tau / 2=0$ and $\omega \tau / 2=\pi$. As a result the $3 \mathrm{~dB}$ bandwidth is decreased.

The changes in frequency response for the noncentered single tum coil compared to the centered coil are most pronounced for $\xi=0$ and $\xi=0.5$. For other values of $\xi$, an internediate response deviation is predicted. In Fig. 6-3 several Faraday response curves are shown for different $\xi$, all with maximum conductor displacement, $\mathrm{X}_{1}=\mathrm{R}$. A comparison can be made with the response for the centered coil, $X_{1} / R=0$, which is also plotied in Fig. 6-3. For values of $\xi>0.5$, symmetry exists in the response predicted by

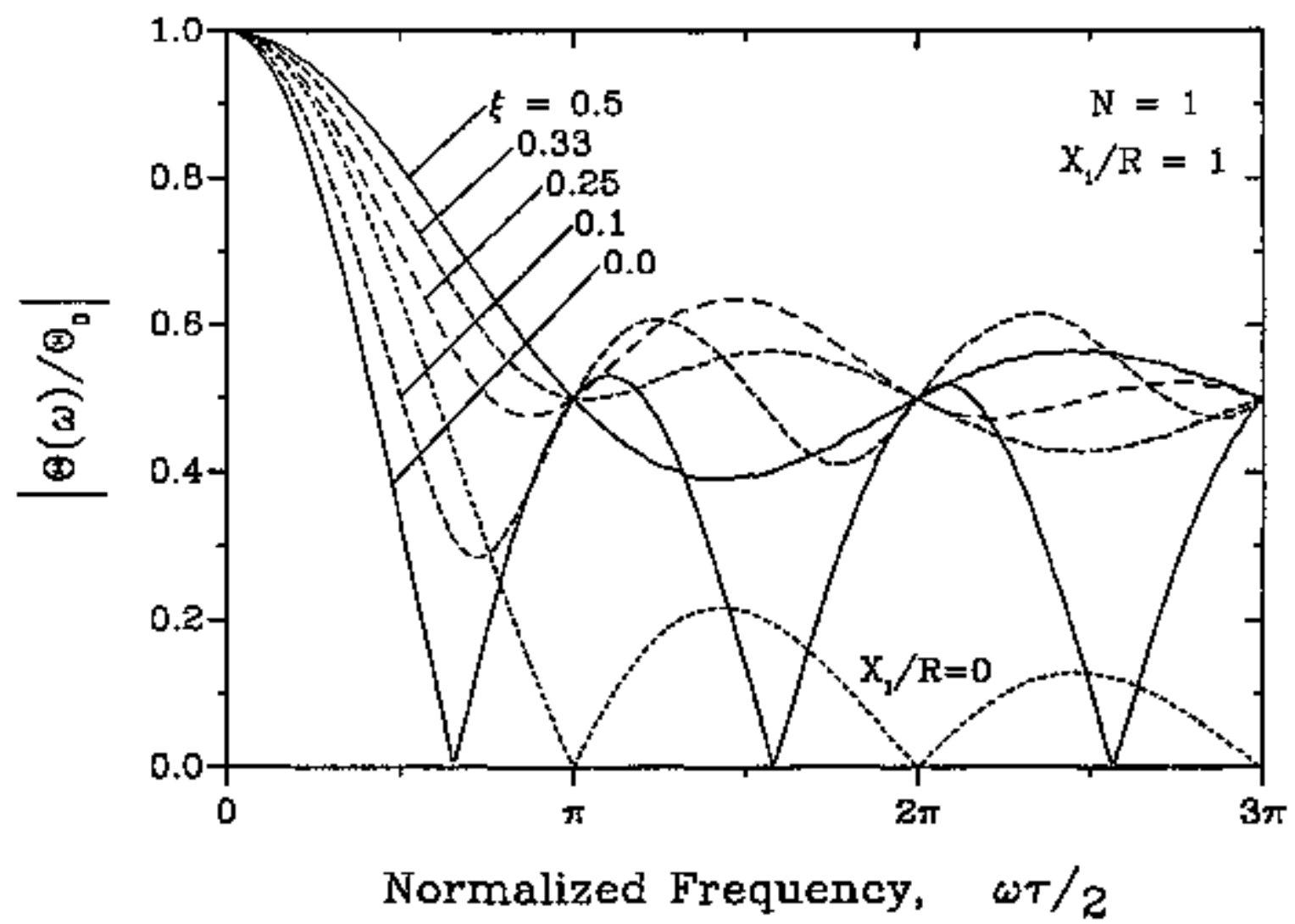

Fig. 6-3. The effects of the light entry point ( $\xi$ ) in the coil on the frequency response for a single tum noncentered, circular coil with maximum conductor displacement $\left(X_{1} / R=1\right)$. The dotted curve is a reference for a centered, circular coil. 
Eq. (6-14): $\Theta(\omega, \xi)=\Theta(\omega, 1-\xi)$ for $0.5<\xi \leq 1$.

Few current sensor applications call for a fiber coil with only one turn; most require multiple tarns for increased measurement sensitivity. When $N>1$ in Eq. (6-14), many of the anomalous features observed in the Faraday response for a single turn are no longer present. Fig. 6-4 shows the predicted relative magnitade of the Faraday rotation for a five tum sensor coil. Notice the cutoff frequency for the noncentered coils, both for $\xi=0$ and $\xi=0.5$, fall at the same frequency as that for the centered coil. This characteristic is seen in the response for all coils with two or more tums; only the single turn noncentered coils have a shifting $\hat{f}_{s 0^{\prime}}$

In Fig. 6-4 resonant responses occur at frequencies where $\omega \tau / 2$ is an integer multiple of $5 \pi$. Here, the five turns in the coil, or the number of close passes between the propagating light in the fiber and the strong field from the conductor dictate the resonant conditions. Also, these resonances occur for the noncentered coil at frequencies where nulls exist in the response for the centered coil. The relative magnitudes for the resonance peaks are consistent with the predictions given by Eq. (6-17). For $X_{1} / R<1$, each higher frequency resonance has a smaller magnitude than the previous one. When $X_{1} / R=1$, the peak magnitudes are approximately $1 / 2$. Close inspection of the plots in Fig. 6.4 shows that $\triangle B$ for the higher frequency resonance lobes is the same as that for the low frequency response lobe.

In Fig. 6-4 the main lobe $3 \mathrm{AB}$ bandwith is indistinguishable from that of the centered coil response regardless of the $X_{1} / R$ or $\xi$ value. This is quite different from that predicted for the single turn coil where significant bandwidth deviations occur. In general, for a fixed $\xi, \Delta B$ changes as $X_{1} / R$ increases (the sign of the deviation in $\Delta B$ depends on $\xi_{j}$ ), and for a fixed $X_{1} / R, \Delta B$ increases as $\xi$ increases from 0 to 0.5 . The change in $\triangle \mathrm{B}$ from that of a centered cołl diminishes rapidly as $\mathrm{N}$ increases. These bandwidth features are shown in Fig. 6-5 for sensor coils with $\mathrm{N}=1,2$, and 3; the curves are plotled only for $\omega t / 2 \leq \pi$ to emphasize the main tobe response. The percen 

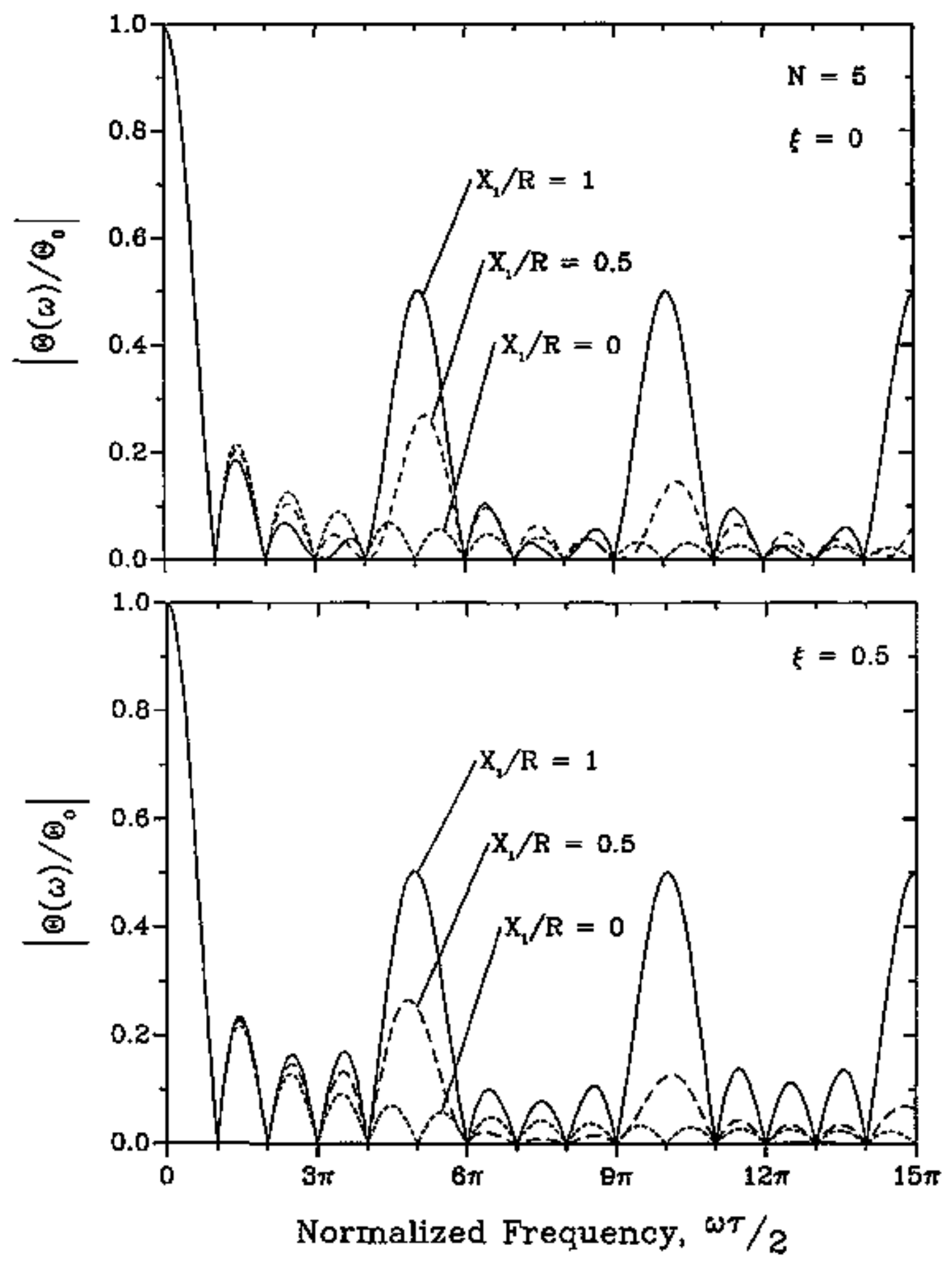

Fig. 6-4. The frequency dependent Faraday response for a five turn circular curtent sensor coil with varying relative axes displacement. Light enters the coil closest to the conductor (top) and farthesl from the conductor (bottom). 


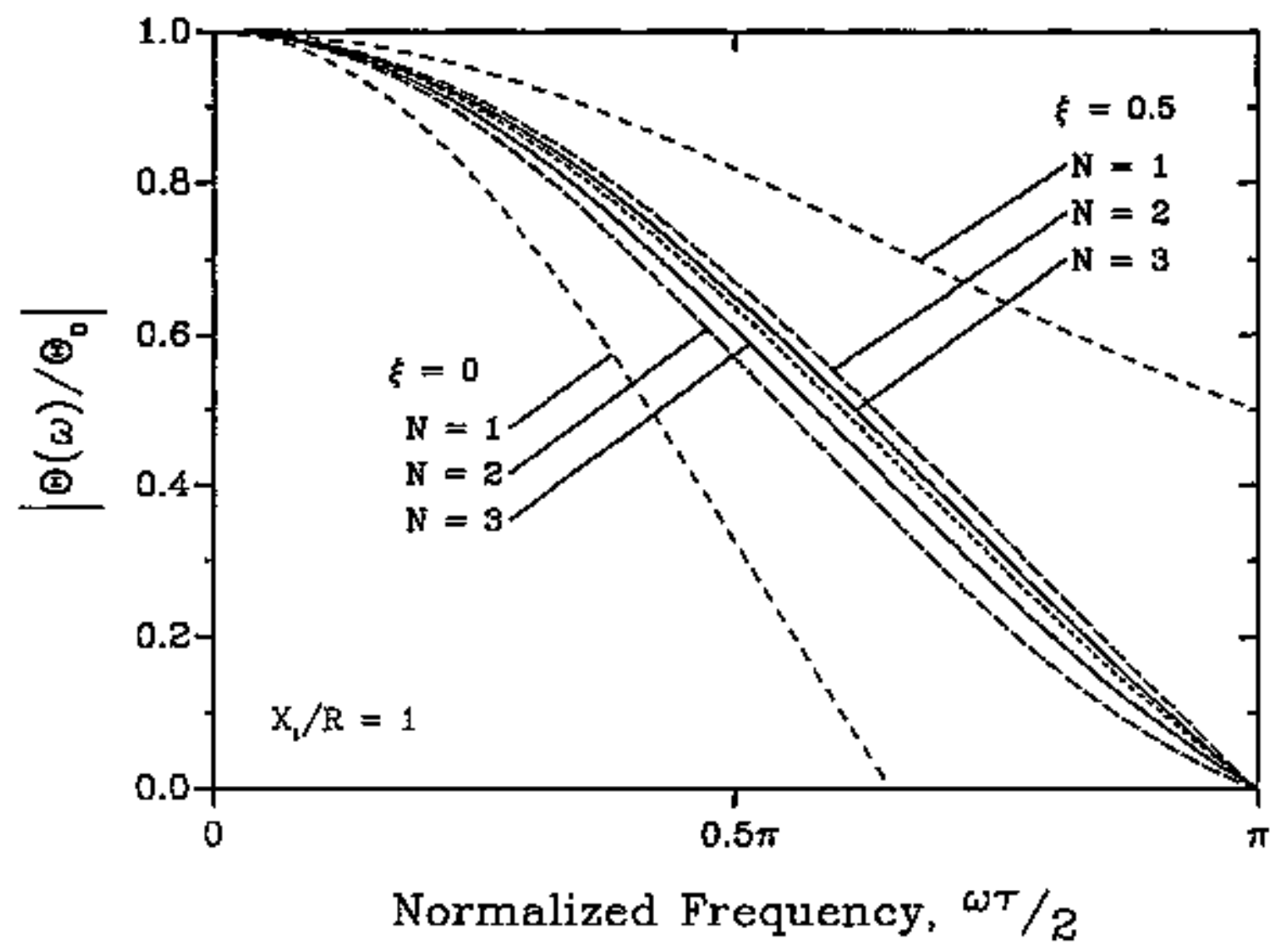

Fig. 6-5. The effects of the number of coil turns and the light entry point on the bandwidth of a noncentered sensor coil with maximum conductor displacement. The dotted reference curve is for a centered, circular coil, $X_{1}=0$. The curve for $\xi=0$ with $\mathrm{N}=1$ is truncated for frequencies above the first nulf for plot clarity.

deviations in bandwidth are also given in Table 6-I for noncentered coil responses with $X_{1} / R=1, \xi=0$ and 0.5 , and for $N$ between 1 and 10 . When $\xi=0$, bandwidths are smaller than those for the centered coils, while with $\xi=0.5$, bandwidths are larger. For a coil with ten turns, the bandwidth deviation is insignificant; $\Delta \mathrm{B}$ can then be estimated using Eq. (5-7) or (5-8) for the centered, circular coil. This indicales that for multitum current sensor coils $(N \geq 10)$, alignment of the coil axis with that of the conductor is not necessary if the range of interest is those freguencies below cutoff.

The frequency response of the noncentered coil is quite sinilar to that of a recirculating tapped fiber delay line [64,65]. As mentioned previous]y, the magnetic 


\section{Table 6-1,}

Changes in the $3 \mathrm{~dB}$ Bandwidth for Noncentered Fiber Coment Sensor Coils $\left(X_{1} / R=1\right)$ Compared to Centered Coils $\left(X_{l} / R=0\right)$.

$\begin{array}{ccc}\mathrm{N} & \text { With } \xi=0.0 & \text { With } \xi=0.5 \\ 1 & -29.1 \% & +50.1 \% \\ 2 & -9.2 \% & +5.8 \% \\ 3 & -4.6 \% & +2.5 \% \\ 5 & -1.9 \% & +0.8 \% \\ 10 & -0.4 \% & <+0.1 \%\end{array}$

fields are largest at points on the fiber coil that are closest to the conductor, and as light propagates in the fjber, it experiences greater Faraday rotation in these regions. Once during each turn this Faraday sotation enhancement occurs; when the freguency of the oscillating field is in phase with this process, a resonant response is observed. This phenomenon is much like the extraction of light on each tum in the tapped oelay line. Resonant response is observed in Eq. (6-14) when $\omega \tau / 2=\mathrm{mN}$. Then the resonant frequencies are $\omega_{\mathrm{R}}=2 \pi \mathrm{m}^{\prime}(\mathrm{N} / \tau)$ or

$$
\mathrm{f}_{\mathrm{R}}=\mathrm{m}^{\prime} \frac{1}{\tau_{1}}
$$

where $\tau_{1}=\tau / \mathrm{N}$ has been previously defined as the transit tinte of light through one coil tum. This resonance frequency relationship has been observed for all Faraday responses predicted for the noncentered coif sensors. An exception ss the response with $\mathrm{N}=1$ and 
$\xi=0.5$; increased responses are observed at the correct higher frequencies, only the antiresonance nulls are missing.

Three responses are plotted in Fig. 6-6 for $X_{1} / R=1$ bot now as a function of a new nomalized frequency, $\omega t_{1} / 2$. With this abscissa, all the higher frequency resonances coincide for sensor coils with varying numbers of turns. The sensor bandwidth still decreases with added fiber turns as expected. This type of behavior has been measured for the tapped fiber delay line with response dependent on the number of fiber taps and the propagation delay between taps [65].

From $\mathrm{Eq}$. (6-17) and Fig. 6-6, the peak magnitudes of the frequency response

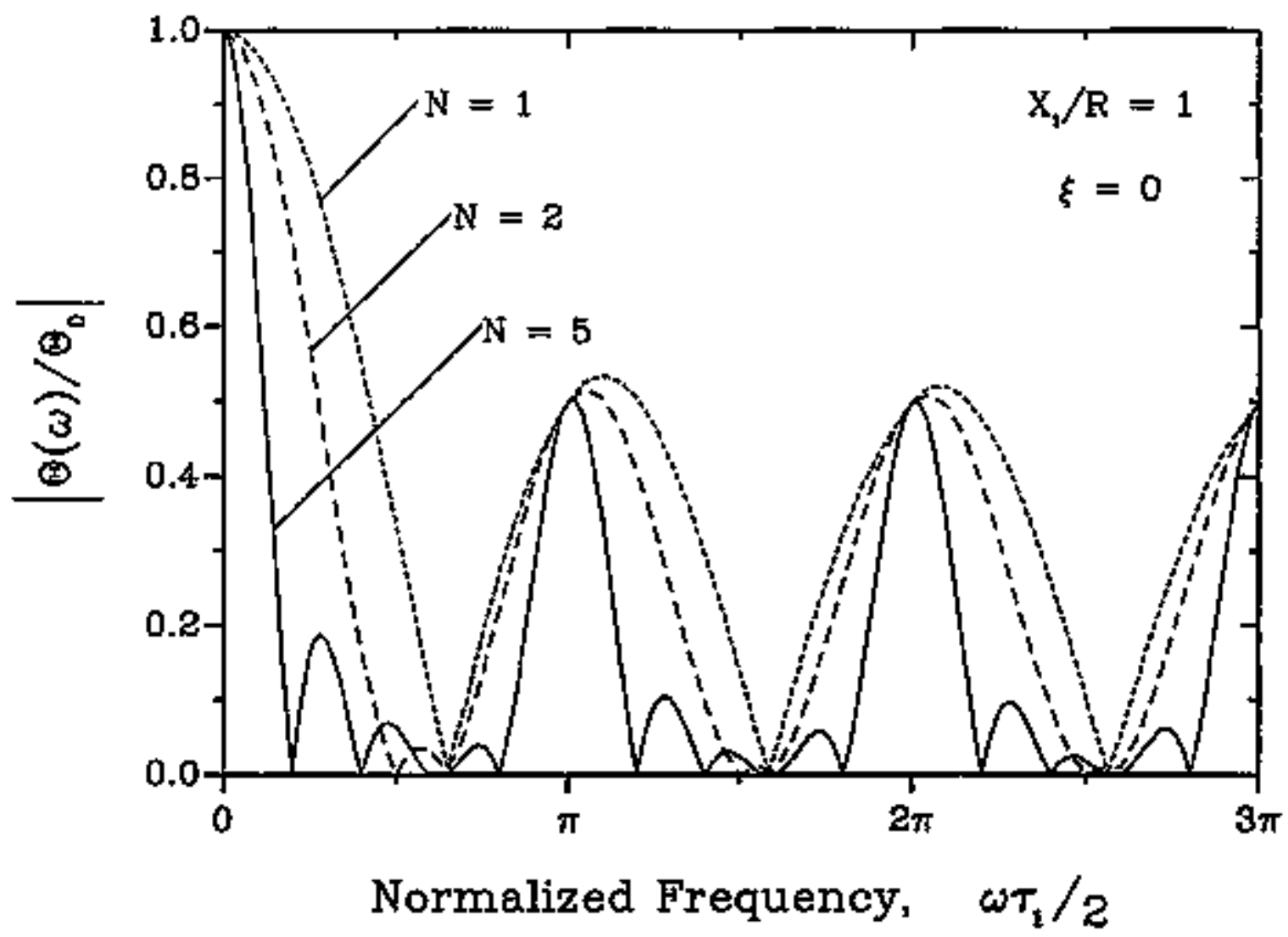

Fig. 6-6. The relative Faraday response for noncentered current sensor coils versus a frequency that has been normalized by the number of turns. Resonances are observed for all three coils at frequencies determined by light transit time in a singte fiber turn. 
resonances are predicted to have a maximum value of approximately $1 / 2$ when the conductor is displaced to the very edge of the coil. This indicates that complete phase matching between the magnetic field propagating along with the conducted current and the light propagating in the fiber does not occur. Enhancements in the Faraday rotation that do occur as the light passes near the conductor once during each revolution around the circular coil are partially negated by out-of-phase interactions that occur when the same propagating light segment is further from the conductor and the magnelic field swings to opposite polarity. Complete phase malching can only occur for true point interactions between the magnetic field and the light, resulting in resonances with relative magnitudes of one. This type of Faraday effect current sensor is described in the nex! chapter of this document.

Equation (6-14) has been used extensively to predicl the magnitudes of the Faraday response for noncentered, circular coils, but it can also be used to determine phase angles. In general, the Faraday sotation should have a frequency dependent phase as expressed in Eq. (5-5) for the centered coil; the phase is a simple function only of the tolal fiber lengh, $\tau$. However, the series sign faclor $(-1)^{2 \mathrm{~m}}$ in Eq. (6-14) can introduce other phase devialions into the response of the displaced coil. If $\xi=0$ or 0.5 , the sign factor is real only, and no deviations occur. But if $\xi$ has other values, the sign factor is complex. The resultant phase shifts are frequency dependent, increasing with $X_{1} / R$, decreasing with $\mathrm{N}$, and obtaining a maximum when $\xi=0.25, \mathrm{~N}=1$ and $\mathrm{X}_{1} / \mathrm{R}=1$. A plot of the frequency dependent phase for this maximum deviation configuration is shown in Fig. 6-7. Throughout the plotted frequency range $(\omega \pi / 2 \leq 3 \pi)$, the phase angle is slightly nonlinear and has a period spproximately $2 / 3$ that for the centered coil.

It is not apparent whether the predicted phase anomalies are real - and observable in measurement systems - or are the result of deficiencies in the model developed to describe the noncentered coil frequency response. A frequency dependent phase shift other than that described by Eq. (5-5) was not anticipated in final 


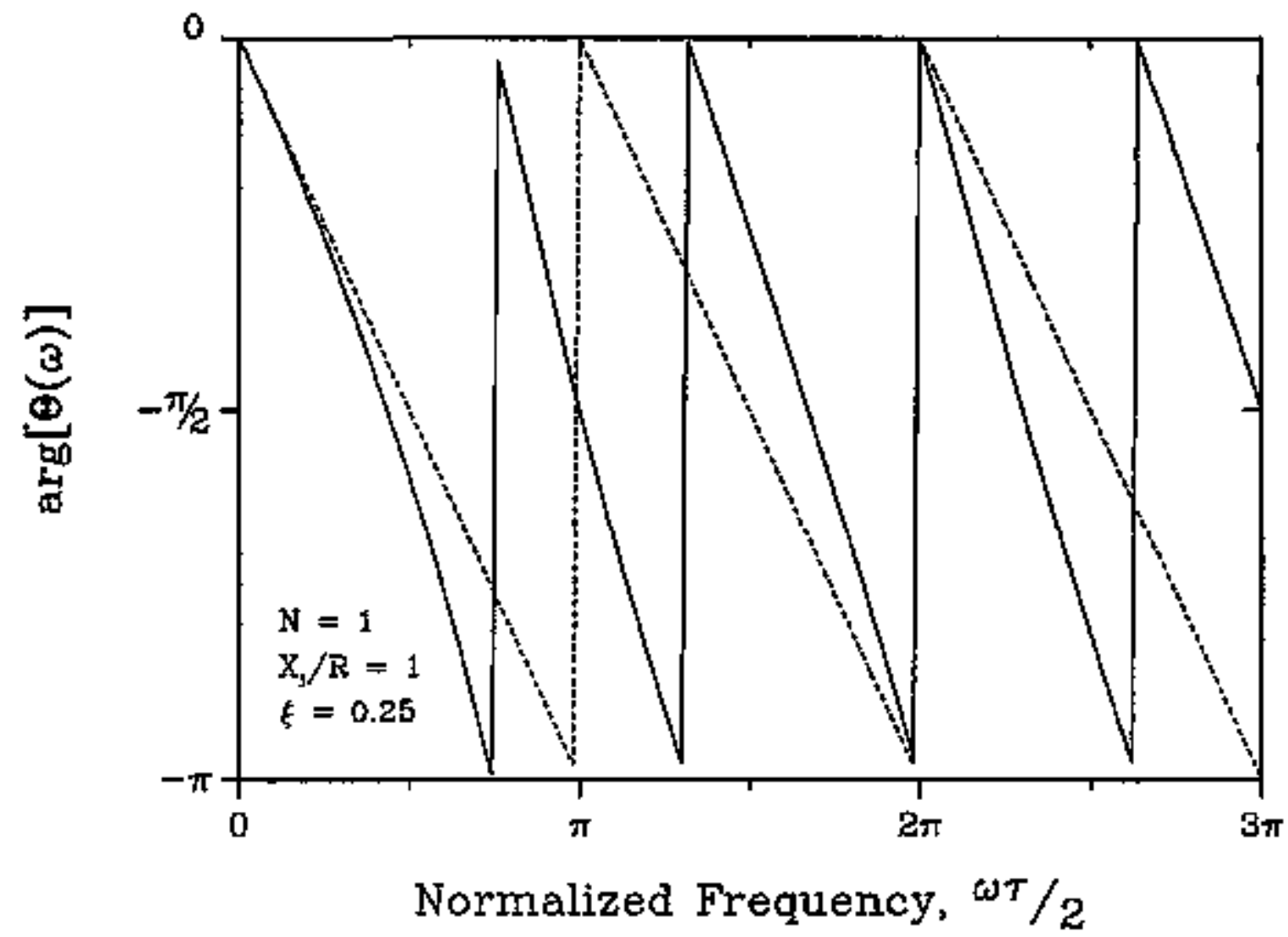

Fig. 6-7. The Faraday rotation phase shift for a single turn coil with maximum conductor displacement and with the light entry point at $90^{\circ}$ to the conductor offset direction. The dotted reference curve is the response phase shift in a centered coil.

computations. A check on the model was conducted by substituting $\cos (\gamma) / \mathrm{r}$ given by $\mathrm{Eq}$. (6-3) directly into Eg- (3-8) and integrating numerically to determine both magnitude and phase. Results identical to those predicted by Eg. (6-14) were obtained. If the model is in error, it exists in a more fundamental assumption. Obviously, more investigations are needed here. In the meantime, these uncertainties limit the capability of the noncentered coil model to predict complete sensor high frequency response functions.

Nonetheless, radially displacing the fiber coil axis from that of the conductor does offer some potential for increased measurement bandwidth and the measurement of currents at higher banded frequencies. Using a single tum coil, significantly increased 
bandwidth and higher frequency response can be achieved by proper placement of the loop. The biggest gain for fiber current sensor applications may be in exploiting the increased response at the higher frequency resonances. In designing such systems, the center frequency of the band is selected by the radius of the coil according to Eq. (6-18). Constraints on minimum radius must be observed to avoid bend bireftingence problems. The Faraday sensitivity of the sensor is then determined by the number of fiber turns in the coil and the maximum amoun of displacement that can be achieved. As observed from the model predictions of Eq. (6-14), the larger the offset, the greater the relative response magnitude in the high frequency band. Response bandwidth is then found from the total fiber length, $\Delta B$ musl be traded off with sensitivity as established by the sensitivity-bandwidth product in Eq. (5-10).

\section{Noncentered CoilMeasurements}

In order to verify some of the predictions of the noncentered coil model, a specific set of parameters was chosen for a coil design and used in the experimental configuration shown in Fig. 4-1. The 1-GHz coaxial airline test cell was selected to generate the appropriate currents. Because of the space constraints in the working volume (outer conductor diameter of $13.3 \mathrm{~cm}$ and inner conductor dianneter of $5.8 \mathrm{~cm}$ ), only a limited range of cois diameters and relative displacement factors can be achieved in this cell. An experimental investigation of the high frequency response predicted for the single turn curent sensor was desired, however, the small signal-to-noise ratio and the timited dynamic range for a one-tum coil in the assembled measurement configuration precluded such a test. A 10 tirn coil with a $9.6 \mathrm{~cm}$ diameter was selected. Parameters of this coil appear as \#7 in Table 5-1. The diameter of the fiber coil was optimized so that one edge touched the cell outer conductor while the opposite edge just wrapped around and touched the cesl center conductor. This coil position gave the maximum-achievable displacement, $X_{1} / R=0.38$, measured from the axis of the center 
conductor. The coil was configured so that fiber leads entered and exited from the same side and then was arranged inside the test cell so $\xi=0$.

The measured frequency response magnitudes for the sensor coil in both the centered and displaced position are shown in Figs. 6-8a and 6-8b, along with the appropriate model-predicted curves. In both plots, experiment and theory agree quite well. The predicted sensor bandwidth is $30 \mathrm{MHz}$ and the cutoff freguency is $67 \mathrm{MHz}$. For the noncentered or displaced coil (Fig. 6-8b), a peak resonance first occurs near 675 $\mathrm{MHz}$, approximately where the tenth null exists for the centered coil frequency response. All the experimental response function peaks and nulls occur at the frequencies predicted.

In Fig. 6-8b, the measured relative magnitude of the resonance peak near 675 $\mathrm{MHz}$ is noticeably smaller than expected from the model. The predicted magnitude is 0.19 , but both experimental data sets indicate a reduced value. This discrepancy could be caused by one or more of the following factors. (1) Data scatter is significant near the resonance peak as seen for the plotted values in Fig. 6-8b. (2) The model neglects the presence of the fiber coil leads, which could be significant in determining frequency response near the resonances. And (3) the model treats the conductor as a filament of moving charge, while in reality the cell conductor has a large diameter and the current tends to concentrate in the outer skin at these high frequencies.

Other key features predicted by the model and shown in Fig. 6-8b are verified by the experiments. One in particular is the missing response lobe just below $600 \mathrm{MHz}$. Also, the ratio of the peak magnitudes for the enhanced responses at $675 \mathrm{MHz}$ and 765 MFlz is quite precise. The absolute magnitudes of the peaks do not agree well with predictions, however, the ratio does. Measurements above $800 \mathrm{MHz}$ were attempted, but these were limited by RF interference in the detection equipment. Fortunately, response information at those high frequencies offers little to the understanding of the model. 


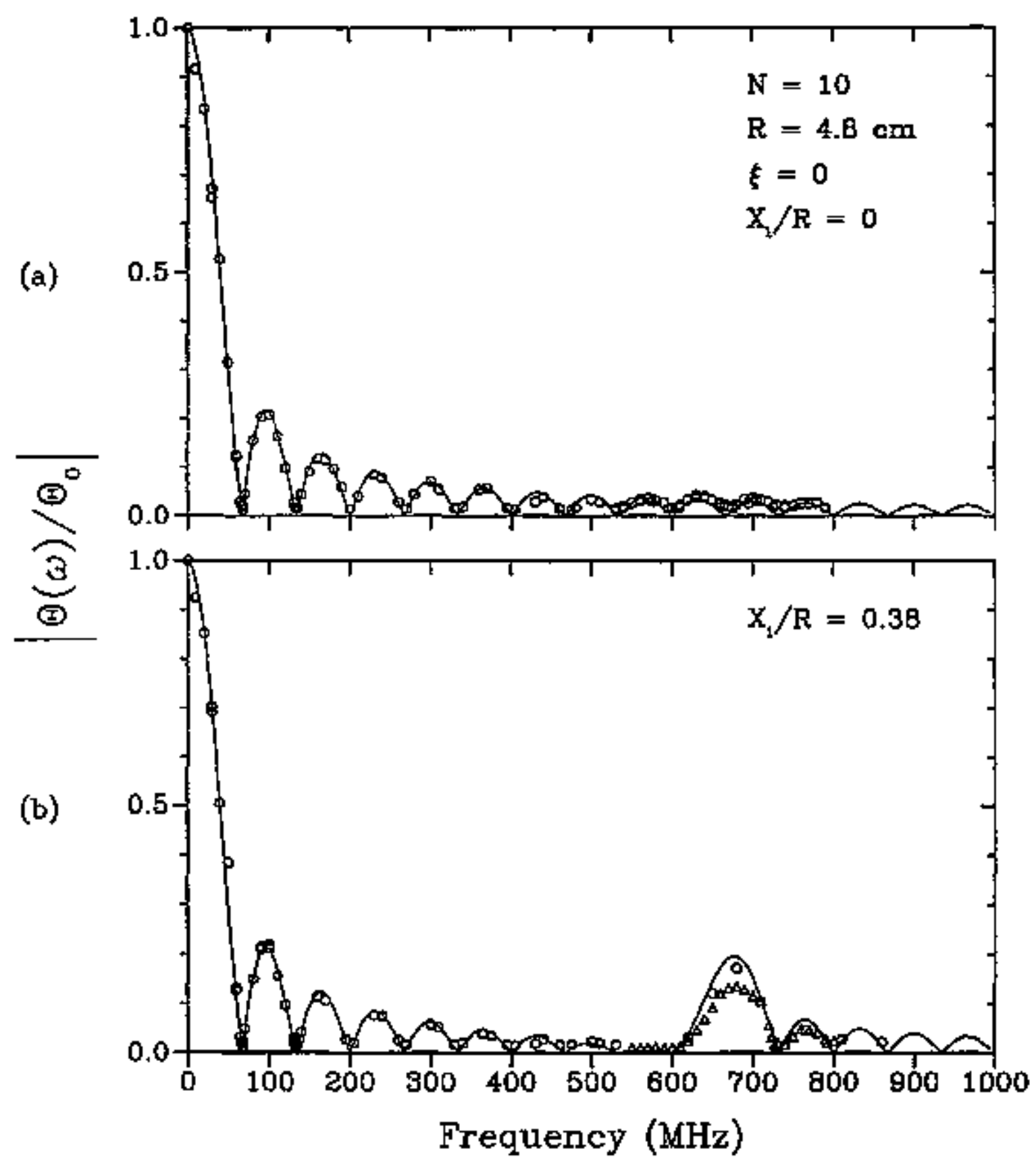

Fig. 6-8. Theoretical and measured frequency response magnitudes for a 10 tum, 9.6 cm diameter fiber cument sensor coil. (a) Measured (points) and predicted (solid curve) responses for the centered sensor coil. (b) Measured (two point curves shown) and predicted (solid curve) responses for the noncentered sensor coil. 


\section{Conductor Outside the Coil}

Ampere's law given by Eq. (2-2) equates the current in a conductor with the mggnetic field strength integrated around any loop enclosing that conductor. For a fiber Faraday current sensor, no response is anticipated at low frequencies when the current conductor does not pass through the coil cross section. At the higher frequencies, though, a response does occur and is addressed here in a brief theoretical discussion.

In the previous model for the noncentered coil, the relative displacement factor was taken to be less than one. However, the expression given by Eq. (6-3) remains valid even for $X_{l} / R$ greater than one. Using this as a starting point, the series expansion is modified and jesults in

$$
\frac{\cos \gamma}{r^{*}}=-\frac{1}{R} \sum_{m=1}^{\infty}\left(\frac{R}{x_{1}}\right)^{m} \cos \left[\mathrm{m}\left(\alpha+\alpha_{0}\right)\right] .
$$

or

$$
\frac{\cos \gamma}{r}=\frac{1}{\mathrm{R}}\left[1-\sum_{\mathrm{m}=0}^{\infty}\left(\frac{\mathrm{R}}{\mathrm{x}_{1}}\right)^{\mathrm{m}} \cos \left[\mathrm{m}\left(\alpha+\alpha_{0}\right)\right]\right] .
$$

These series are complementary to Eq. (6-5) and converge for $\mathrm{X}_{1}>\mathrm{R}$. When Eq. (6-19) is substituted into Eq. (3-8) and integrated over the transit time through the total fiber, the frequency dependent Faraday rotation is found to be

$$
\Theta(\omega)=\Theta_{0}\left[\frac{\sin (\omega t / 2)}{(\omega t / 2)}\right] e^{-j \omega t / 2} \sum_{m=1}^{\infty}(-1)^{2 m \xi}\left(\frac{R}{x_{1}}\right)^{m}\left[1-\left(\frac{m N \pi}{\omega t / 2}\right)^{2}\right]^{-1}
$$

This expression for the rotation is very similar to Eq. (6-14). Resonant responses occur at the same higher frequencies. One major difference is the absence of the $\mathrm{m}=0$ tem in the series of Eq. (6-21); this previously was found to be a necessary condition for the 
existence of the low frequency response. Thus when $\omega \approx 0, \theta(0)=0$ for all $\mathrm{N}$ as expected.

The relative magnitude determined from $\mathrm{Eq} .(6-21)$ is plotied versus the nomalized frequency, $\omega \tau / 2$, in Fig. $6-9$ for two current sensor coil configurations: (a) a single tum with $\xi=0.5$ and (b) five turns with $\xi=0$. For both configurations no responses occur at zero frequency, but secondary lobes and resonant peaks are present at the higher frequencies. As the sensor coils are moved further away from the conductor, the response magnitudes decrease at all frequencies as expected. Eventually as $X_{1} / R$ grows larger, the conductor and coil are separated by such a large distance that magnetic fields are too smali to induce any significant Faraday rotation in the fiber.

In Fig. 6-9a the effects of the light entering and exiting the fiber coil farthest from the conductor $(\xi=0.5)$ are again illustrated. As discussed previously in conjunction with Fig. 6-2b (conductor inside the coil), a single tom coil will exhibit a continuous and enhanced higher frequency response with the proper positioning of the conductor and the coil. The Faraday response magnitude for the solid $\left(X_{1} / R=1\right)$ and dashed curves in Fig. $6-9 \mathrm{a}$ are symmetric about the magnitude $1 / 2$. The frequency response with the conductor just outside the coil is simply one minus that response when the conductor is just inside the coil. This phenomenon is the result of the sign change that occurs in $\mathbf{B} \cdot \mathrm{dl}$ for that fiber section just adjacent to the conductor. It is also evident in the frequency dependent angle/distance relationships, Egs. (6-5) and (6-20), which are simple complements when $X_{1}=R$.

In Fig. 6-9b, the characteristic resonance responses for the noncentered coils are again observed. The center frequencies of the resonant bands fall at $0 \pi / 2=\mathrm{m} / \mathrm{N} \pi$, exactly the same as those predicted for a coil with $X_{1}<R$. Resonance bandwidths are again determined by the total fiber length. If $X_{1} / R=1$, the peak relative magnitudes are all approximately $1 / 2$, but when $X_{1} / R>1$, each successively higher frequency resonance is weaker than the previous one. 

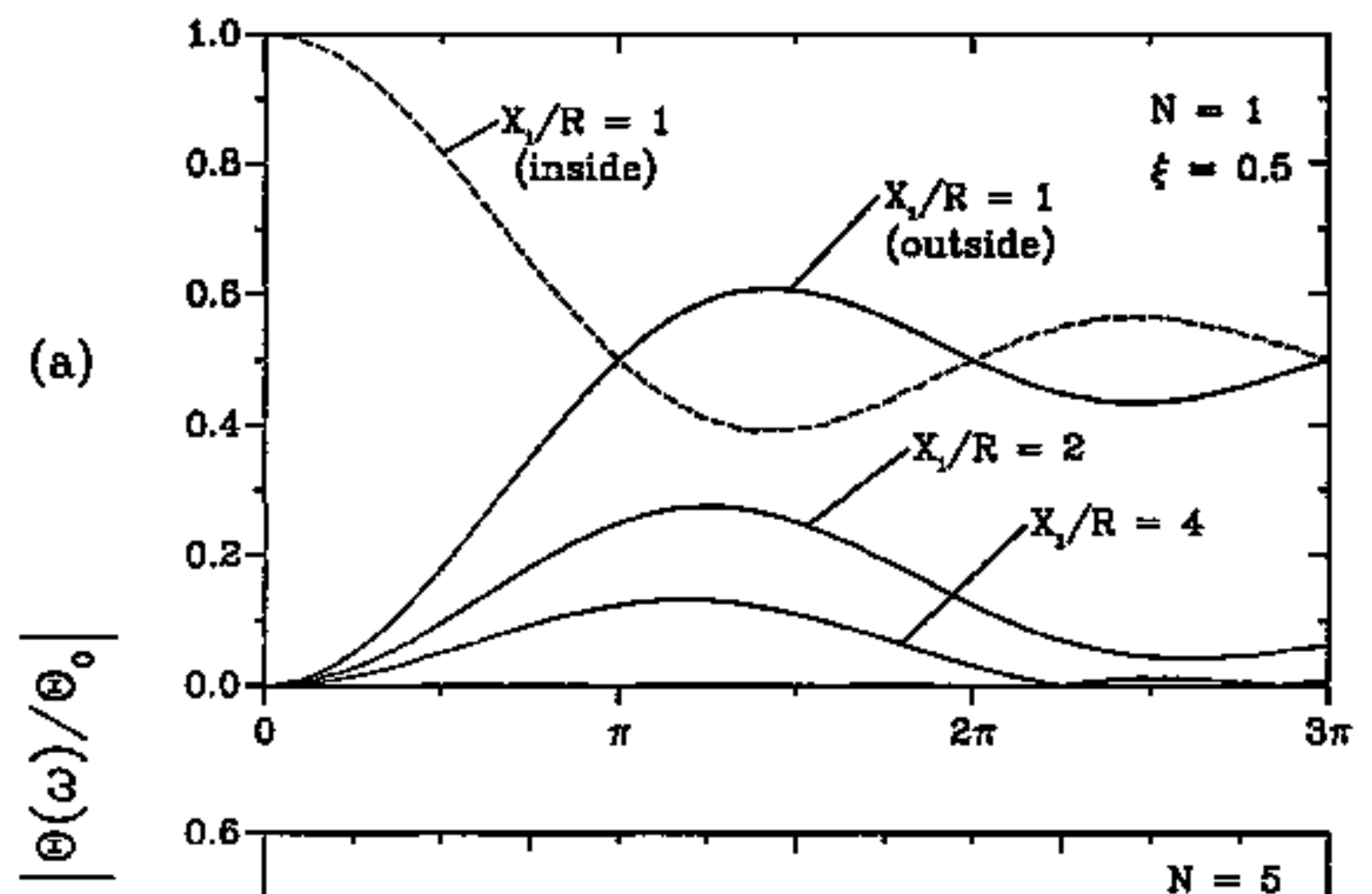

(b)

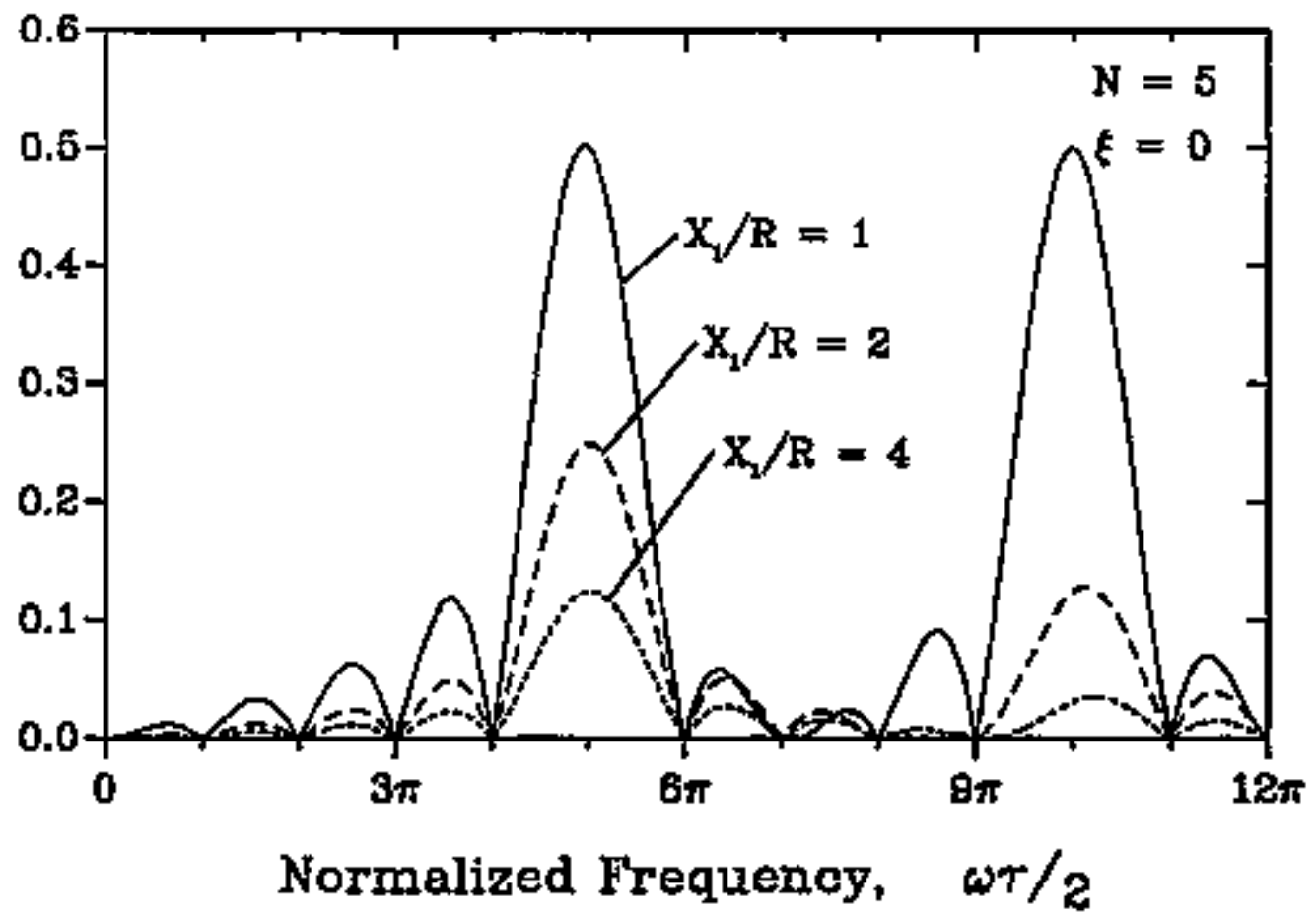

Fig. 6-9. The theoretical high frequency Foraday rotation relative magnitude for fiber current sensor coils with the conductor outside the coil cross section $\left(X_{1} / R \geq 1\right)$. (a) A single turn coil with light entering farthest from the conductor, and (b) a five turn coil with light entering nearest the conductor. 
This model of a conductor external to the fiber sensor coil was not verified experimentaily. The frequency range for the TEM mode in the $50 \Omega$ coaxial airline test cells is not compatible with the expected first resonance frequency or other significant features predicted by the model. Experimentation may be possible in a modified test cell, but for now this concept remains a theoretical curiosity. 


\section{CHAPTER 7}

\section{COILS WITH NONCIRCULAR CROSS SECTION}

The shape of the planar coil cross section can produce changes in the high frequency Faraday response much like the resonant enhancements created by displacement of the coil and conductor axes. In this chapter, the Faraday rotation is investigated for coils with noncircular cross section, and the effects at the higher freguencies are observed. Coils with two particular geometries are studied: (1) those with a highly elongated cross section and the current condnctor fully displaced to one edge of the fiber coil, and (2) those with a rectangular cross section and the conductor either centered or displaced within the coil. The first geometry represents a unique situation since llie magnetic freld interacts oniy with a small segment of the fiber in each tum. Essentially complete phase matching can occur for this interaction; and the resulting "delta function" Faraday response produces higher frequency resonances with peaks approaching relative magnitudes of one. This response is in contrast to the magnitude timit of approximately $1 / 2$ for the noncentered, circular cross section coil discussed in the previous chapter. The rectangular coil geometry provides a simple framework for investigating frequency responses for ali configurations of coils, those with centered or displaced conductors and those with varying degrees of cross section eccentricity. Also, predictions from the rectangular coil models are easily extrapolated to other coils of arbitrary cross section. Only theoretical studies were possible for the rectangular coils, but experimental verifications of the delta function interaction were performed using two specific coil implementations.

\section{Delta Function Response Model}

A highly elongated fiber sensor coil is shown in Fig. 7.1a, In the limit, the conductor can be treated as a point soarce with the fiber loop making a tight bend just 
around the conductor. Realistically, this contiguration is impossible since mechanical limilations prohibit such bends and the bend-induced birefringence would be extremely large. But theoretically, this coil cross section is ideal: Faraday rotation occurs only for light in that segment of the fiber adjacent to the conductor. At all other points on the fiber coil, the magnetic field is orhogonal to the light propagation and zero response is recorded. Because this interaction occurs in a small spatial region, TEM mode constraints on the current-produced field can be relaxed; but for the purpose of extending the results of this model to more practical coil configurations, the fields along the conductor are assumed to be planar.

Figure 7-1b illustrates the ideal path followed by the tight propagating in the fiber. When the fiber passes near the conductor, it is assumed to make a light,

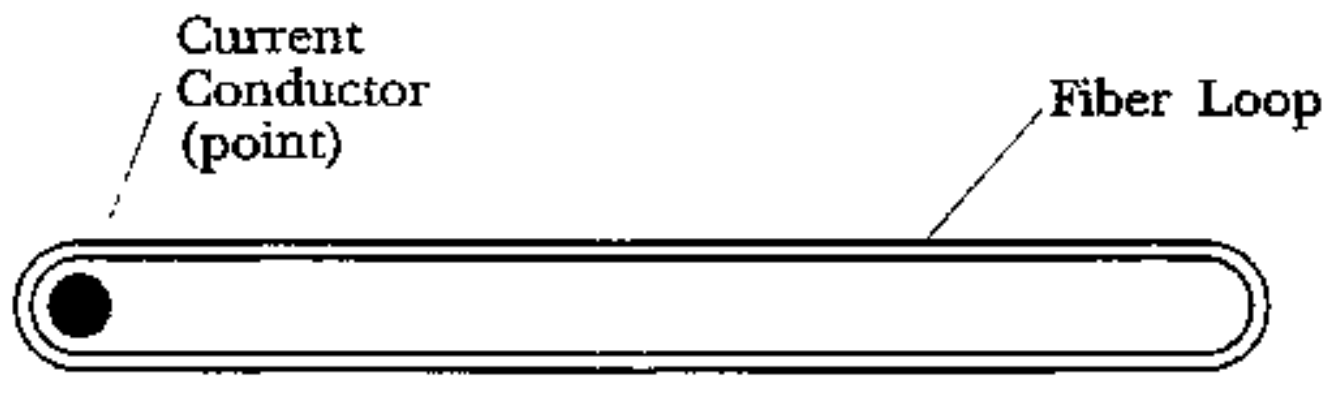

(a)

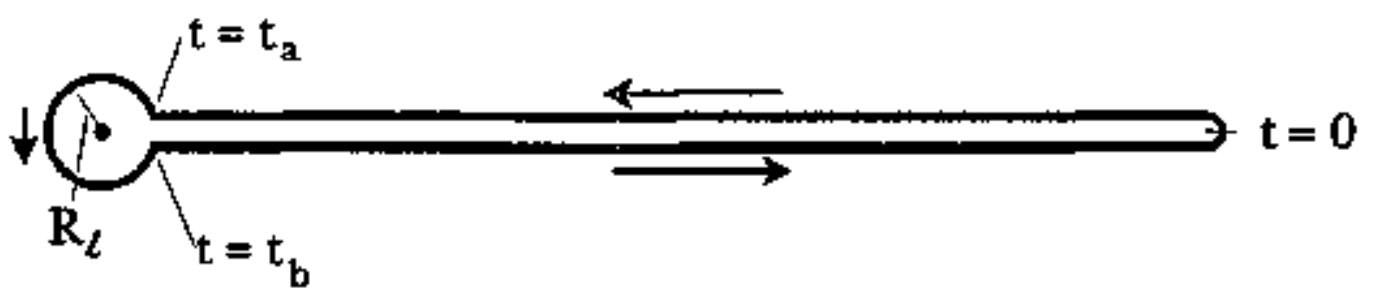

(b)

Fig. 7-1. The delta function response fiber current sensing model. (a) The current conductor is fully displaced inside a highly elongated fiber coil cross section. (b) The malhematical treatment of the light path. 
approximately circular loop at a constant radial distance $\mathbf{R}_{\ell}$ from the point conductor. The magnetic flux density in this short segment of fiber is then given by Eq. (3-1) with $\mathrm{r}=\mathrm{R}_{t}$ and $\mathrm{k} \cdot 1=0$. The field is assumed always to be codirectional with the propagating light in the fiber loop, so that the local interaction is described by

$$
B \cdot d l=\mu_{f}^{i} e^{-j \omega t} \frac{d l}{2 \pi R_{\ell}}
$$

Al points along the siraight segments of the fiber coil (away from the circular bend) shown in Fig. 7-1b, the general field-light interaction is described by

$$
B \cdot d l=B \cos (\gamma) d l \text {. }
$$

which is Eq. (3-2) with $\psi=\pi / 2$. In these straight segments, however, $\gamma$ is always taken to be $\pi / 2$, and $B \cdot d l=0$. Then, substituting Eq. (7-1) into Eq. (2-1) and converting to time-dependent integralion yields

$$
\Theta(\omega)=\frac{\mu_{f} V i}{2 \pi R_{\ell}}\left(\frac{c}{n}\right) \int_{t_{b}}^{t_{b}} e^{-j \omega t} d c
$$

which describes the frequency dependent Faraday rotation in a single turn of the elongated fiber current sensor. The integration times, $t_{a}$ and $t_{b}$, are as indicated in Fig. 7-1b. In a multiturn coil, the fiber will make $\mathrm{N}$ passes by the conductor. The total Faraday rotation then becomes the sum of that for each individual interaction. Also, during each pass the integration times mest be appropriately delayed to account for variations in the oscillating magnetic field. In general, $t_{\mathrm{b}}=m \tau_{1}+\xi \tau_{1}-\tau_{z} / 2$ and $t_{\mathrm{b}}=m \tau_{\mathrm{l}}$ $+\xi \tau_{1}+\tau_{i} / 2$ for $\mathrm{m}=0$ to $N-1 ; \tau_{t}=2 \pi \mathrm{R}_{l}(\mathrm{n} / \mathrm{c})$ is the transit time of the light through 
each stuall, circular segment, and $\boldsymbol{\xi}_{\boldsymbol{}_{1}}$ is an arbitrary time delay from the entry point of the light in the fiber (Identical to that introduced in Eq. (6-11)). The Faraday rotation for the $\mathrm{N}$-turn elongated coil is given by

$$
\Theta(\omega)=\frac{\mu_{f} V i}{2 \pi R_{\ell}}\left(\frac{c}{n}\right) \sum_{m=0}^{N-1} \int_{m \tau_{1}+\xi_{\tau_{1}}-\frac{\tau_{\ell}}{2}}^{m \tau_{1}+\xi \tau_{1}+\frac{\tau_{\xi}}{2}} e^{-j \omega t} d t
$$

Proceeding with the integration leads to

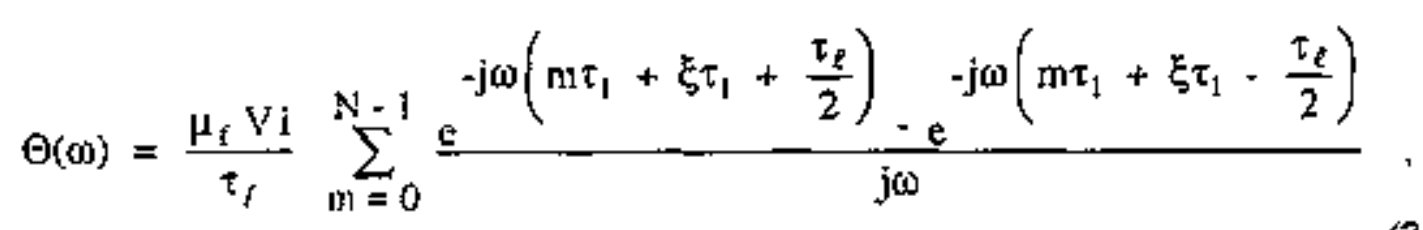

and a final solution of

$$
\Theta(\omega)=\frac{\Theta_{0}}{N} e^{-j \omega \xi \tau_{1}} \frac{\sin \left(\omega \tau_{c} / 2\right)}{\left(\omega \tau_{i} / 2\right)} \sum_{m=0}^{N-1} e^{-j \omega m \tau_{1}} .
$$

The low frequency response, $\theta_{0}$ (\$) for the entire coil is given by Eq. (2-3).

The concept of a point interaction between the magnetic field and the propagating light is now introduced by allowing $\tau_{t} \rightarrow 0$. The factor $\sin \left(\omega \tau_{t} / 2\right) /\left(\omega \tau_{t} / 2\right)$ in Eq. (7-6) becomes unity, and an expression for the "delta function" Faraday response results:

$$
\theta_{\delta}(\omega)=\theta_{0} e^{-j \omega \xi \tau_{i}} \frac{1}{N} \sum_{m=0}^{N-1} e^{-j \omega m \tau_{1}}
$$

The time delay created by the light entry point produces only a phase delay in the final 
rotation, which, in this model, has no impact on the magnitude of the Faraday rotation. In fact, the arbitrary light entry point is significant only if it occurs exactly at the conductor so that $\mathrm{N}+1$ interactions are produced for $\mathrm{N}$ loops in the coil. This is quite different from the delay introduced in the noncentered, circular coil model where the light entry point had a large impact on the magnitude of the Faraday response for all values of $\xi$. In order to avoid the confusion in this delta function model between the number of close passes the light makes by the conductor and the number of turns in the coil, the light is assumed to enter at or near the zero location indicated in Fig. 7-1b. Then $\mathrm{N}$ will represent both quantities. Consequently, $\xi=1 / 2$ in $\mathrm{Eq}$. (7-7), and

$$
\Theta_{\delta}(\omega)=\Theta_{0} e^{-j \omega \tau_{1} / 2} \frac{1}{N} \sum_{m=0}^{N-1} e^{-j \omega m \tau_{1}}
$$

Or, since $\tau_{\mathrm{t}}=\mathrm{t} / \mathrm{N}$,

$$
\Theta_{\delta}(\omega)=\theta_{0} e^{-j \omega \tau / 2 N} \frac{1}{N} \sum_{m=0}^{N-1} e^{-j m \omega \tau / N} .
$$

These expressions for the fregaency dependent Faraday rotation can also be derived by simply treating the fueld-light interaction as a series of delta functions separated in time by the transit time of the light through a single coil turn, $\tau_{1}$, and delayed in time by $\xi_{\tau_{1}}$ :

$$
B \cdot d l=\mu_{f} i e^{-j \omega t} \sum_{m=0}^{N-l} \delta\left(t-m \tau_{1}-\xi \tau_{1}\right) d t
$$

When Eq. (7-10) is substituted into Eq. (2-1), the equivalent time integral becomes 


$$
\Theta_{5}(\omega)=\Theta_{0} \frac{1}{N} \sum_{m=0}^{N-1} \int_{0}^{t} \delta\left(t-m \tau_{1}-\xi \tau_{1}\right) e^{-j \omega t} d t
$$

Completing the integration yields Eq. (7-7). This treatment is much simpler, however, it is less elegant than the initial approach and lacks the generality needed for later developments.

If a fiber current sensor makes only a single pass by the conductor, the magnitude of the Faraday response from $\mathrm{Eq} .(7-9)$ is $\left|\Theta_{8}(\omega)\right|=\left|\Theta_{0}\right|$, and an infinite bandwidth results. This prediction is expected from the definition of the delta funclion interaction. For two passes by the conductor,

$$
\Theta_{8}(\omega)=\Theta_{0} \cos \left(\frac{\omega \tau}{4}\right) e^{-j \omega \tau / 2} .
$$

For a coil with $\mathrm{N}$ loops, a general expression for the response magnitude is

$$
\left|\Theta_{\delta}(\omega)\right|=\left|\Theta_{0}\right| \frac{1}{N} \sqrt{\left[\sum_{m=0}^{N-1} \cos \left(\frac{m \omega \tau}{N}\right)\right]^{2}+\left[\sum_{m=0}^{N-1} \sin \left(\frac{m \omega \tau}{N}\right)\right]^{2}},
$$

and the phase angle is

$$
\phi=-\tan ^{-\mathrm{t}}\left[\frac{\sum_{m=0}^{N-1} \sin \left[\left(m+\frac{1}{2}\right) \frac{\omega \tau}{N}\right]}{\sum_{m=0}^{N-1} \cos \left[\left(m+\frac{1}{2}\right) \frac{\omega \tau}{N}\right]}\right] .
$$

Equation (7-13) is plotted in Fig. $7-2$ for $N=2$ and $N=5$. The plot abscissa is the normalized frequency, $\omega \tau / 2$. Response resonances occur when $\omega \tau / 2$ equals a multiple 


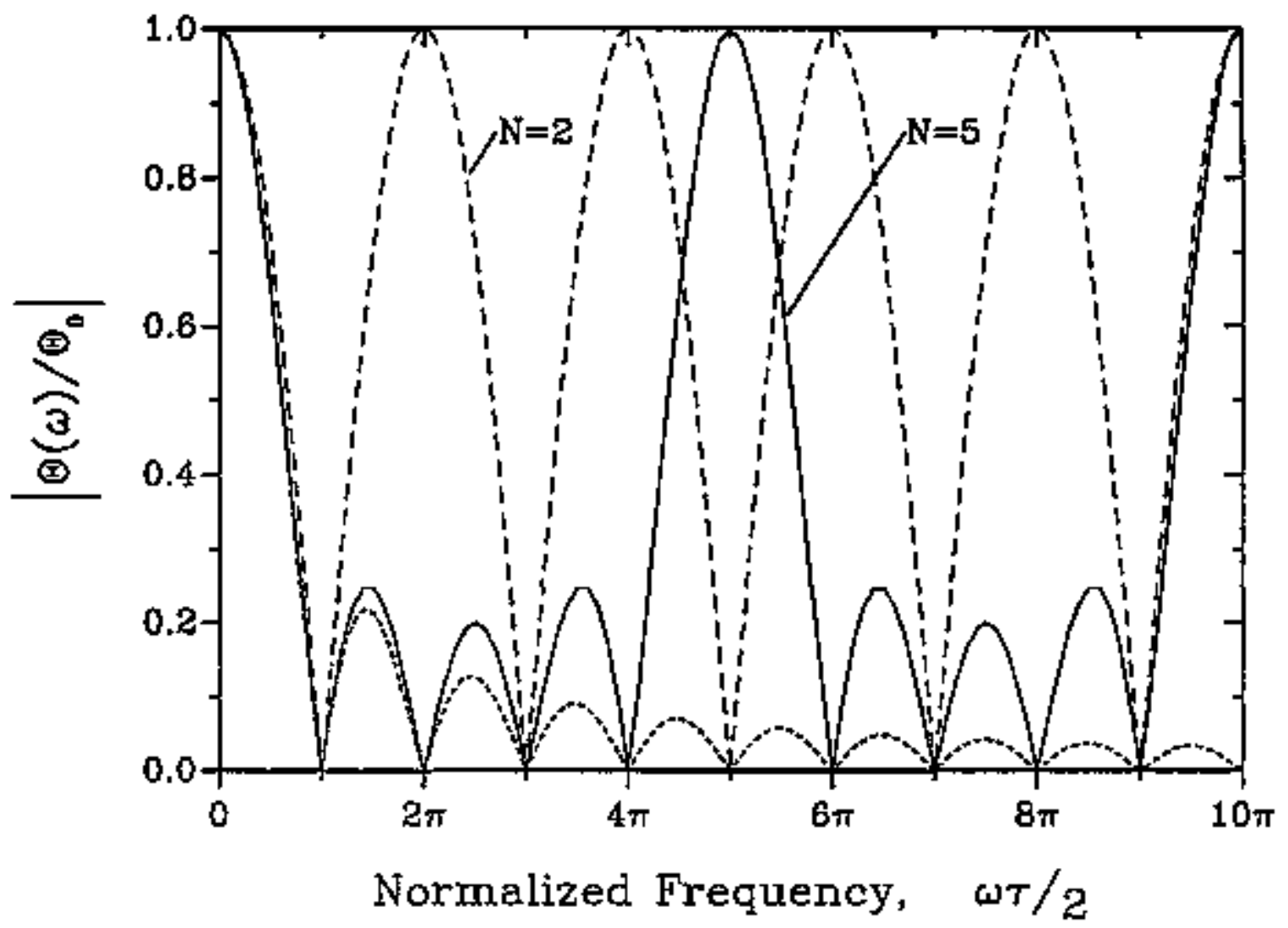

Fig. 7-2. The Faraday rotation relative magnitude versus normalized frequency for delta function current sensor coils with two and five turns. The dotted reference curve is the response for a centered, circular coil.

of $N \pi$, with the number of intervening nulls equal to $N-1$. The response magnitudes in Fig. 7-2 for $\mathrm{N}=5$ resemble those for the noncentered, circular coil, Fig. 6-4, except higher frequency resonances now have a unity relative magnitude instead of $1 / 2$. This unity magnitude results from complete phase matching between the magnetic field and the propagating light at each of the single-point interactions. As light in the fiber propagates away from the conductor, it experiences no further out-of-phase rotations that reduce the resonance magnitude as in the noncentered, circular coil. Also observed in Fig. 7-2 that is not seen in Fig. 6-4 is the complete frequency symmetry of the lobes and nulis about each resonance - each rising and falling freguency edge is symmetric about 
the lobe/resonance center frequency. The bandwidths of the main response lobe and each of the higher frequency resonance lobes are also equal.

Equation (7-13) can also be plotted with the normalized frequency $\omega \tau_{1} / 2$ as the abscissa; such a plot appears in Fig. 7-3. The similarities between Figs. 6-6 and 7-3 are obvious. The responses illestrated in Fig. 7-3 are identical to those previously discussed for the lapped fiber delay line for $\mathrm{N}$ fiber taps (see Chapter 6). Comparisons can be made to similar figures in Ref. 65.

The Faraday response bandwidths for coils configured for a delta function interaction can be significantly larger than those for the centered, circular coils made

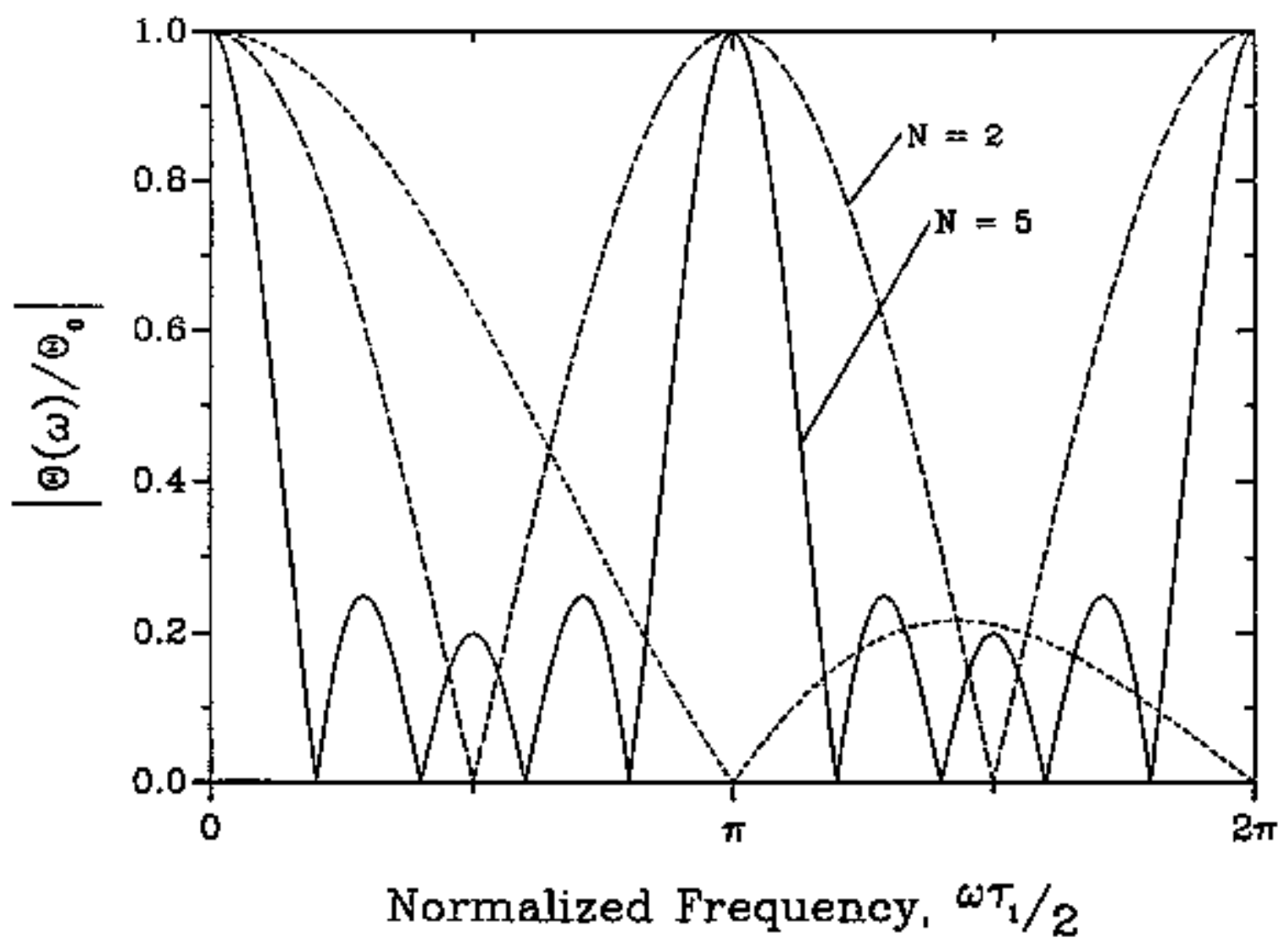

Fig. 7-3. The relative magnitude of the Faraday rotation from Fig. 7-2 replotted versus the normalized frequency $\omega \tau_{1 / 2}$. The dotted reference curve is the response for a centered, circular coil. 
with the same length of fiber. This is obvious for a single loop coil which produces a theoretically infinite bandwidth. In Fig. 7-2, a notable difference in bandwidth is observed for the $\mathrm{N}=2$ curve compared to the centered, circular coil response even though the cutoff freguency for the two curves is identical. Using Eq. (7-12), the $3 \mathrm{~dB}$ response point for $\mathrm{N}=2$ will occur when

$$
\cos \left(\frac{\omega_{u} \tau}{4}\right)=\frac{I}{\sqrt{2}} .
$$

Then

$$
\Delta B=\frac{c}{4 \pi R \pi N},
$$

which is farger than the bandwidth for the centered, circular coil, Eq. (5-7), by $12.8 \%$. As $\mathrm{N}$ increases the bandwidth for the delta function coil quickly approaches that for the centered, circular coil. Extracting data from the numerical computations of Eq. (7.13), the bandwidth increases are $5.1 \%$ for $\mathrm{N}=3,1.3 \%$ for $\mathrm{N}=5$ and negligible when $\mathrm{N} \geq 7$. These bandwidth deviations can be compared to those of the noncentered, circular coil in Table 6-1 for the same value of $\mathrm{N}$; larger response bandwidihs result from the point interactions.

Based on the predictions from this delta function model, as well as those from the noncentered coil model, a general statement can be made about fiber conrent sensor coil configurations: for sensors with large numbers of fiber turns, the Faraday response in the low frequency main lobe is unaffected by displacement or coil cross-sectional shape; only sensor response at the higher frequencies is modified.

Although it is not obvious from a first look, the response phase given by $\mathrm{Eq}$. $(7-14)$ can be reduced to $-(\omega t / 2$ for all vajues of $N$. This is easily identified for $\mathrm{N}=2$ by inspecting Eq. (7-12). As a further example, if $\mathrm{N}=3$, Eq. (7-14) is rewritten as 


$$
\phi=-\tan ^{-1}\left[\frac{\sin \left(\frac{\omega \tau}{6}\right)+\sin \left(\frac{\omega \tau}{2}\right)+\sin \left(\frac{5 \omega \tau}{6}\right)}{\cos \left(\frac{\omega t}{6}\right)+\cos \left(\frac{\omega \tau}{2}\right)+\cos \left(\frac{5 \omega \tau}{6}\right)}\right] \text {. }
$$

The sine functions are expanded into

$$
\sin \left(\frac{\omega \tau}{6}\right)=\sin \left(\frac{\omega \tau}{2}-\frac{\omega \tau}{3}\right)=\sin \left(\frac{\omega \tau}{2}\right) \cos \left(\frac{\omega \tau}{3}\right)-\cos \left(\frac{\omega \tau}{2}\right) \sin \left(\frac{\omega \tau}{3}\right)
$$

and

$$
\sin \left(\frac{5 \omega \tau}{6}\right)=\sin \left(\frac{\omega \tau}{2}+\frac{\omega \tau}{3}\right)=\sin \left(\frac{\omega \tau}{2}\right) \cos \left(\frac{\omega \tau}{3}\right)+\cos \left(\frac{\omega \tau}{2}\right) \sin \left(\frac{\omega \tau}{3}\right) .
$$

Then Eq. (7-17) becomes

$$
\begin{aligned}
\phi & =-\tan ^{-1}\left[\frac{2 \sin \left(\frac{\omega \tau}{2}\right) \cos \left(\frac{\omega \tau}{3}\right)+\sin \left(\frac{\omega \tau}{2}\right)}{2 \cos \left(\frac{\omega \tau}{2}\right) \cos \left(\frac{\omega \tau}{2}\right)+\cos \left(\frac{\omega \tau}{2}\right)}\right]=-\tan ^{-1}\left[\frac{\sin \left(\frac{\omega \tau}{2}\right)\left[1+2 \cos \left(\frac{\omega \tau}{3}\right)\right]}{\cos \left(\frac{\omega \tau}{2}\right)\left[1+2 \cos \left(\frac{\omega \tau}{3}\right)\right]}\right] \\
& =-\tan ^{-i}\left[\tan \left(\frac{\omega \tau}{2}\right)\right]=-\frac{\omega \tau}{2} .
\end{aligned}
$$

Determining the phase in this mathematical fashion for larger $\mathrm{N}$ guickly becomes unwieldy, but the solution is always the same, $\phi=-\omega \tau / 2$. This phase is identical to Eq. (5-5) for the centered, circular coil indicating that phase in this sensor configuration is also dependent only on the total Jight transit time or the total fiber length.

\section{The Complette Response Model}

Based solely on the delta function interaction, the Faraday response resonances displayed in Fig. 7-2 would extend unatienuated (at unity) to infinite frequency. Such a 
system, of course is not physically realizable, and the previons presentation of the delta function response model must be considered only for its conceptual value. A more realistic model is constructed by recognizing that $\theta_{8}(0)$ in Eq. (7-9) is the transfer function for an ideal fiber current sensor having $N$ point interactions. The transfer function is then used to construct complete frequency response functions for physical systems. In general, for each pass of the fiber near the conductor, the interaction length between the magnetic field and the propagating light in the fiber may be small but is finite. The two propagating electromagnetic signals become convolved over this short interaction Iength. Then in the frequency domain, the Faraday response for the coil system is nothing more than the transfer function, Eq. (7-9), multiplied by the expected frequency response for the short fiber length while making a single pass.

If the short interaction length is given as $\ell$, the function $\theta_{f}(\omega)$ describes the response for a single interaction. The complete frequency dependent Faraday rotation, which depends on the number of passes $N$, is then the product of two functions,

$$
\Theta(\omega)=\Theta_{\delta}(\omega) \cdot \Theta_{\ell}(\omega)
$$

The function $\Theta_{\alpha}(\omega)$ can be labeled an envelope function, since in the frequency domain, it bounds the resonant response due to $\Theta_{8}(\omega)$ alone. If a functionat form for $\Theta_{2}(\omega)$ can be identified or computed for a single pass of the fiber by the conductor, then the rolloff in the magnitudes of the higher frequency resonance peaks can be established.

By inspecting Eq. (7-6) and comparing it to Eq. (7-19), one functional form for the envelope function, $\Theta_{\lambda}(\omega)$, can be determined. The function $\sin \left(\omega \tau_{t} / 2\right) /\left(\omega \tau_{t} / 2\right)$ is a direct result of using a short circular fiber loop in the derivation of the model for the highly elongated coil. And as would be expected, this function is equivalent to Eq. (5-3), less the phase factor, when the appropriate transit time $\tau_{t}=(\mathrm{n} / \mathrm{c}) \ell$ is used. Approximating the short interaction length with a circular loop is often a good estimate 
for many fiber current measurement systems. In particular, sach a description allows for quick computations of the magnitudes of the resonance peaks since they fall under a wejl-defined enveloping function.

Using Eq. (7-19), the frequency characteristics of the conplete response function for a multiturn coil in a delta function configuration are determined by three separate parameters of the fiber curtent sensor coil. All of these parameters are related to welldefined lengths of different fiber sections and can be used in the design of optimum sensor coils. (1) The resonance peaks in the response function occur at higher frequencies (see Eq. (6-14)) related to propagation through one loop of the coil, $L_{1}=(c / n) \tau_{1}$. (2) The bandwidth of the main response lobe and the bandwidths of all the unattenuated higher frequency resonance peaks are found from the total length of fiber in the coil, $\mathrm{L}$. And (3) the enveloping magnitude of the resonance peaks is determined by the shon interaction length, $e$, of a single pass of the fiber by the conductor. For most applications that exploit the advantages of the delta function response model, the relationship between these three lengths will be $\ell \ll \mathrm{L}_{1} \leq \mathrm{L}$.

Interesting phenomena result from use of the complete response function in Eq. (7-19) when $\ell$ is not small compared to $L_{1}$. A trivial result occurs for $\mathrm{N}=1$ in $\Theta_{6}(\omega)$; $\Theta(\omega)=\theta_{\ell}(\omega) e^{-j \omega \tau / 2}$ as expected, regardless of the value of $\ell$. Bat if $N>1$, the formulation of Eq. (7-19) provides a shortcut for determining the frequency response for more complicated coil systems, especialty those that use double-passireflective geometries or series of identical coils. As an example, assume a moltiturn, circular coil is used and one lead is terminated such that the light is reflected back through the coil. Light injection and detection are both accomplished at the nonreflecting lead. Such a current sensor coil configuration is used in high sensitivity systems to reduce the effects due to reciprocal interactions such as stress or temperature-induced birefringence changes. If the coil is centered about the conductor, $\theta_{l}(\omega)$ is simply $\sin \left(\omega \tau_{l} / 2\right) /\left(\omega \tau_{l} / 2\right)$, for $\tau_{\varepsilon}=2 \pi R_{\ell} N_{e}(n / c)$ the one pass transit time through the entire coil, $N_{c}$ tums. The delta 
function response accounts for phase delays in the light propagation. Here $\mathrm{N}_{\S}$ (number of light passes) $=2$ and $\Theta_{\delta}(\omega)$ is given by Eq. (7-7) with $\xi=0$ and $\tau_{\mathrm{l}}=\tau_{\mathrm{d}}$, the time reguired for light to propagate from the middle of the coil, $\ell / 2$, to the reflection point and back. By definition, $\tau_{d} \geq \tau_{\boldsymbol{r}^{*}}$ The complete Faraday response is then

$$
\Theta(\omega)=\theta_{0}\left[\frac{\sin \left(\omega \tau_{\ell} / 2\right)}{\left(\omega \tau_{\ell} / 2\right)}\right] \cos \left(\frac{\omega \tau_{d}}{2}\right) e^{-j\left(\omega \tau_{d} / 2\right)} .
$$

Similar descriplions of the Faraday response can be derived for other sensor systems.

\section{Demonstration of the Model}

The delta function response model was verified using the two fiber coil configarations shown in Fig. 7-4. Both experimental systems used the coaxial test cell \#2 listed in Table A-1. This cell hass an inner conductor diameter of $2.5 \mathrm{~cm}$, an outer conductor diameter of $5.75 \mathrm{~cm}$, and a HOM cutoff freg̨uency of $2.3 \mathrm{GHz}$ [34]. All coils had 10 loops of fiber with each loop being approxtanately two meters in length and with a small portion of each loop passing through the cell. The Faraday response is then limited to the interactions with light in those short segments of fiber inside the cell; outside the cell, no field exists and the propagating light experiences no induced rotation. In the first configuration (Fig. 7-4a), the fiber made a short tarn around the cell center conductor entering and exiting the cell at the same point. This simulates very closeiy the light path illustrated in Fig. 7-1b. In the second configuration (Fig. 7-4b), a straight frber section passed near the center conductor with the fiber entering and exiting on opposite sides of the cell. The various lengths in the fiber coil were chosen to achieve a narrow response bandwidth and to place several of the resonance peaks at frequencies that were within the measurement range of the experimental instumentation. 


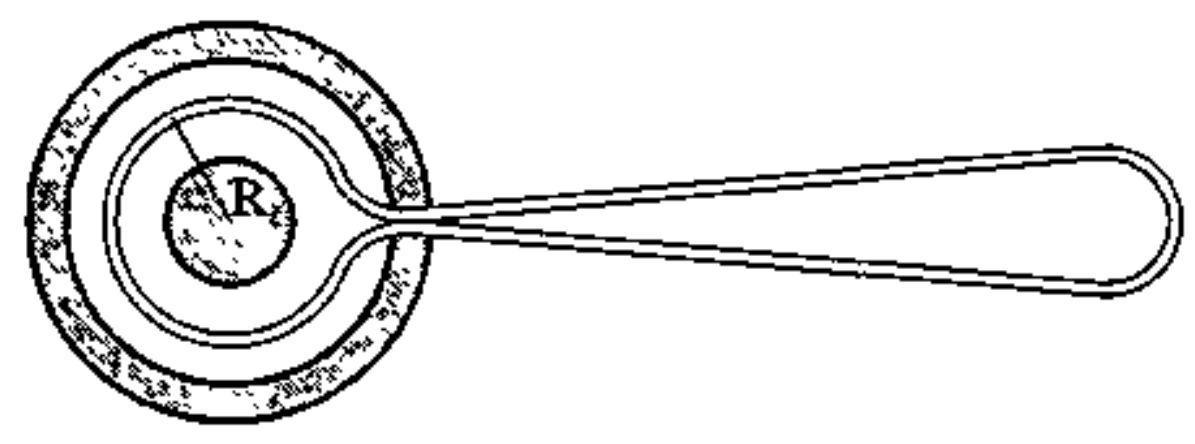

(a)

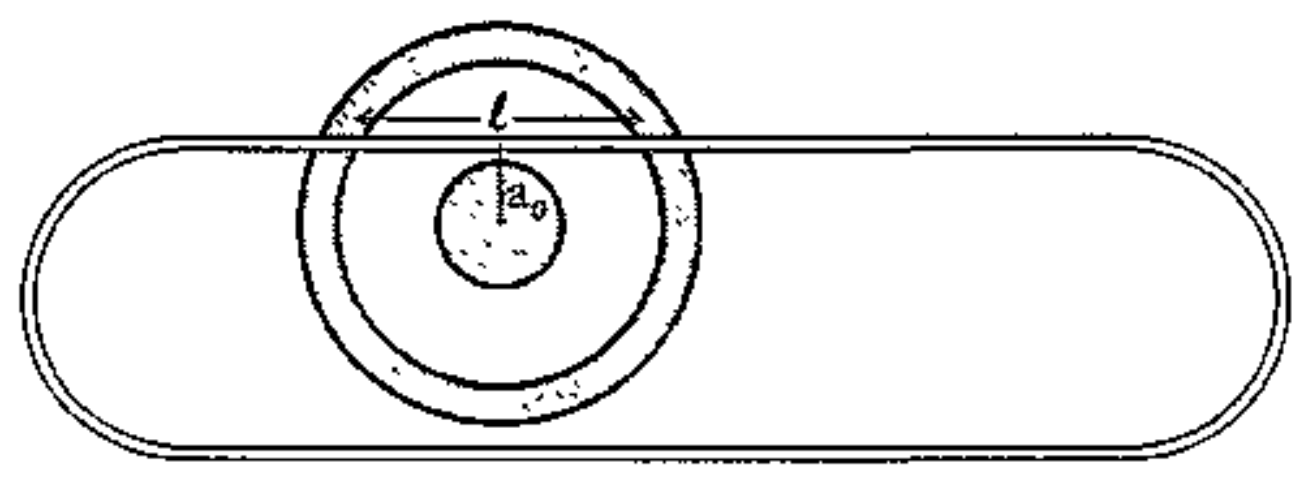

(b)

Fig. 7-4. Two fiber coil configurations used to demonstrate the delta function response model.

The relative magnitude of the Faraday rotation measured using the first experimental configuration is shown in the two graphs of Fig. 7-5. In Fig. 7-5a the model response, depicted by a solid curve, and the experimental results, shown as the open circles, are plotted for the lower frequencies. The resonance peaks are spaced about $101 \mathrm{MHz}$ apart, the bandwidths are approximately $4.5 \mathrm{MHz}$ and the cutoff frequency occurs at $10.1 \mathrm{MHz}$. Although the model and experimental curves in Fjg. 7-5a agree well for the frequencies near resonance, the responses at the intervening frequencies do not agree at all. The secondary lobe magnitudes and the null freguencies do not match. Symmetry of the measured values about the resonance frequencies, however, is preserved. 


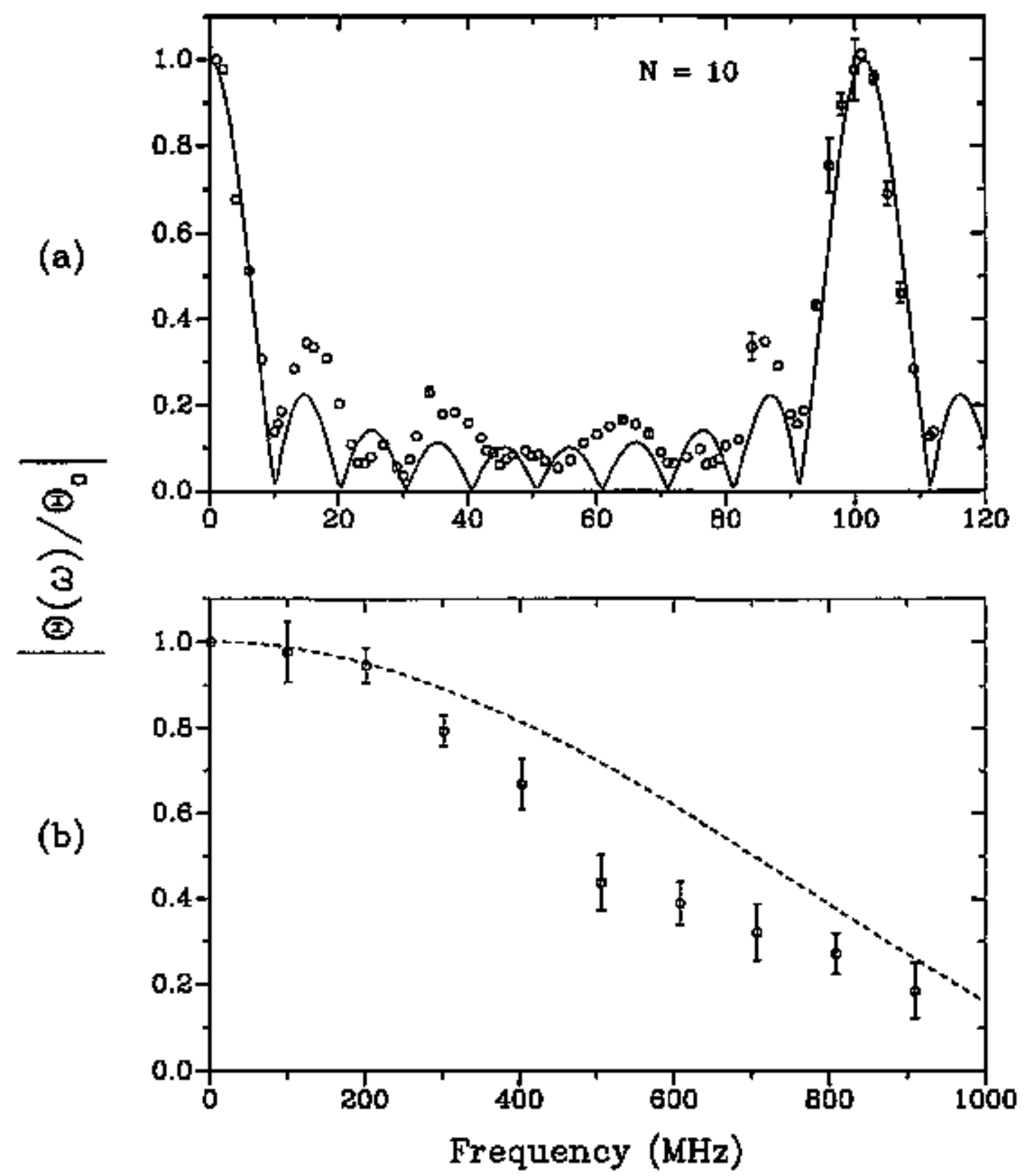

Fig. 7-5. The measured (open circles) and predicted Faraday response at low frequencies (a) and higher frequencies (b) for a 10 turn test coil with short tight loops around the conductor. The solid curve in (a) represents the theoretical behavior near and below the first higher frequency resonance. In (b), the dashed curve is an estimate of the response of an enveloping function for the short fiber length interaction, and the plotted points are measured values only at frequencies near the resonance peaks. Error bars represent total scatter in the measured values. 
Much of this disagreement between measurement and model is attributed to bendinduced linear birefringence created by the tight tams as the fiber loops around the cell center conductor. This coil was prepared using the twisted and rejacketed fiber with 40 twistsin [29], which should have been adequate for testing at this diameter, but apparently when tight bends are introduced, the fiber jacket breaks free from the glass and the twist relaxes. By modifying the bend radius of the loops within the cell, significant changes were noted in the frequency response. The curves plotted in Fig. 7-5 represent the best agreement between the model and the experiment. Although the presence of tinear birefringence in the fiber is besieved to produce perturbations in the frequency response of the Faraday rotation, a detailed investigation of this phenomenon goes beyond the present scope of this study.

The finite interaction length of the short fiber loop inside the test cell causes a rolloff of the resonance peak magniludes at the higher frequencies. This feature is seen in Fig. 7-5b. The experimental data are plotted only at the peaks of each resonance. The totled curve is a rough estimate of the envelope function computed using the magnitude $\left|\sin \left(\omega \tau_{l} / 2\right) /\left(\omega \tau_{l} / 2\right)\right|$ fron Eq. (7-6). Although the 20 toops of fiber inside the cell are not circular in cross section, an average length is estimated for these fiber segments and used to detemine a value for $\tau_{f}$. As seen in Fig. $7-5 \mathrm{~b}$, the experimental data are bound by relatively large error bars, especially at the higher frequencies. These uncertainties, coupled with some residual linear bireftingence effects in the fiber and the imprecise estimates used for the model envelope function, make quantitative comparisons of the curves in Fig. 7-5b difficult. The general trend in the measured data, however, follows the prediction.

The second experimental configuration (Fig. 7-4b) was designed to remove the interfering effects from bend-induced linear birefringence. This was achieved by osing only short, straight fiber segments passing through the cell without any bending. Results from measurements using this arrangement are shown in Fig. 7-6 along with the model 
predictions. The measured and calculated responses near the resonance freguencies agree well, just as they did for the first configuration. In this case, however, the measured secondary lobe magnitudes and the null frequencies between the resonances are much closer to those predicted by the model. Exact identification of the nulls was difficult with this type of current monitor since a $10 \mathrm{~dB}$ loss in sensitivity occurs. This sensitivity loss results from having only a partial tum of optical fiber inside the cell acting as the sensor, whereas in the first configuration, a full sensor tum around the center conduclor existed.

A nice feature of this second experimental configuration is that it lends itself to a relatively simple compulation of the complete response function. Each of the ten straight sections of singlemode fiber passing through the cell will experience a rotation given by

$$
\theta_{c}(\omega)=\frac{\Theta_{0}}{\tau_{c}} \mathrm{a}_{0} \int_{0}^{\tau_{c}} \frac{\mathrm{e}^{-\mathrm{j} \omega \mathrm{l}}}{\mathrm{a}_{0}^{2}+\left(\frac{\mathrm{c}}{\mathrm{n}}\right)^{2}\left(\frac{\tau_{l}}{2}-\mathrm{t}\right)^{2}} \mathrm{~d} \mathrm{t},
$$

where $\tau_{l}=(\mathrm{n} / \mathrm{c}) \mathrm{e}$ is the transit lime of light through the straight section and $\mathrm{a}_{0}$ is the distance of closest approach the fiber makes with the axis of the cell center conductor. Here $\Theta_{0}=\mathrm{VB}_{0} \ell$, where $\mathrm{B}_{0}$ is an arbitrary static flux density created by the current at unit distance from the conductor. Equation (7-21) must be normalized to the low frequency response to remove the dependence on $\mathrm{B}_{0}$ and other geometric parameters. Integrating for $\omega=0$ results in

$$
\Theta_{\ell}(0)=2 \frac{\Theta_{0}}{\tau_{\ell}}\left(\frac{n}{c}\right) \tan ^{-1}\left(\frac{c \tau_{\ell}}{2 \mathfrak{n} a_{0}}\right) .
$$

The normalized Eq. (7-21) is integrated numerically to produce the envelope function, 


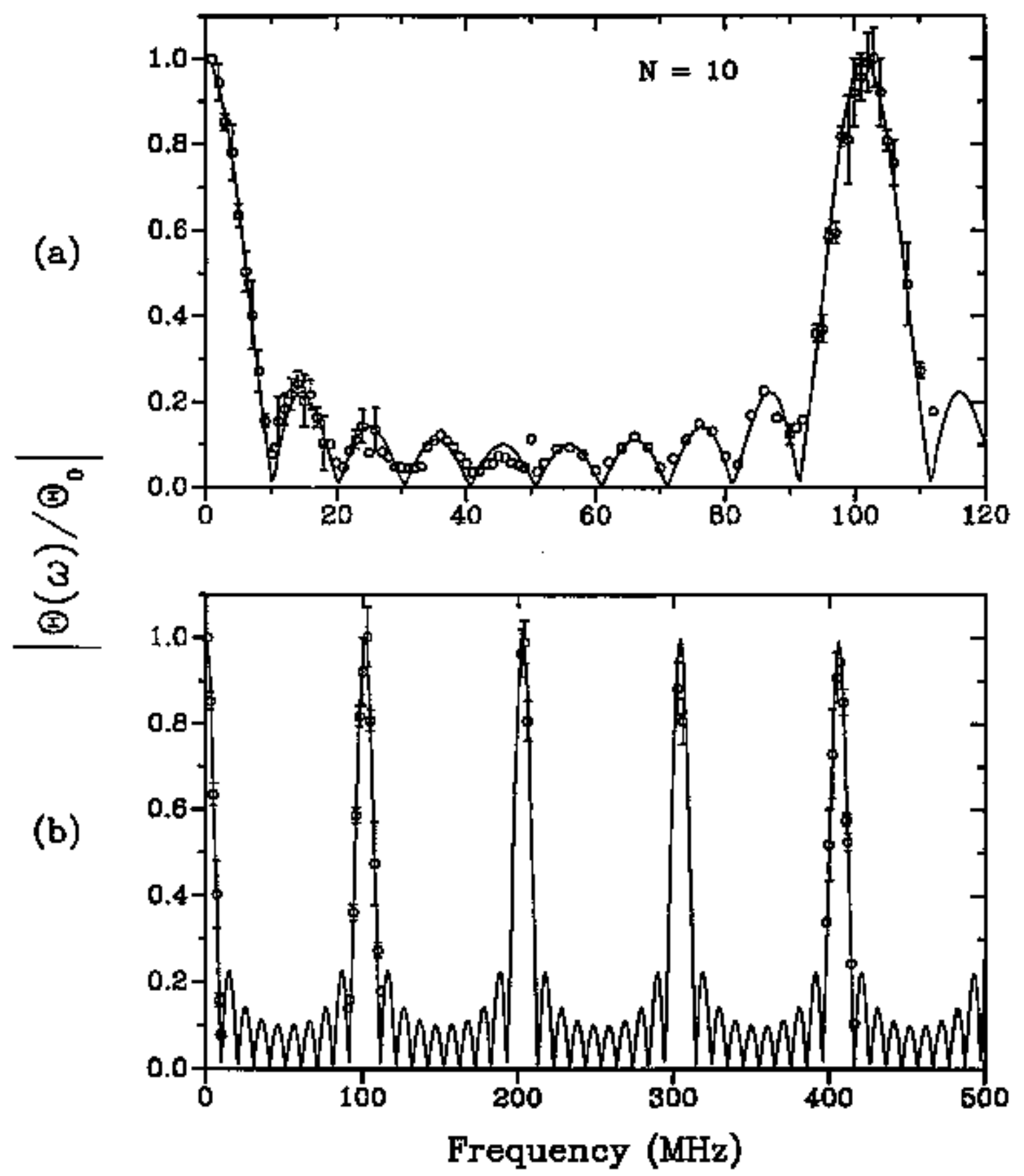

Fig. 7-6. The measured (open circles) and predicted (solid curves) Faraday response at low (a) and higher (b) frequencies for a test coil with short straight segments passing near the conductor. Only measured values near the resonance peaks are plotted in (b). Enor bars on the measured values represent total data scatter. 
and then substituted into $\mathrm{Eg}$. (7-19). The resulting high frequency model response is f used to generate the solid curves in Fig. 7-6. Resonance frequencies, bandwidths and cutoff frequencies predicted by this model are identical to those of the first experimental coil configuration. These features are expected since they depend only on $\tau$ and $\tau_{1}$ (or equivalently on $\mathrm{L}$ and $\mathrm{L}_{\mathfrak{l}}$ ) which are identical for both coils. The high freguency rolloff of the resonance peak magnitudes, however, is dependent on $\tau_{t}$ and $\mathrm{a}_{0}$ in Eq. (7-21). Here, $\mathrm{a}_{0}=1.52 \mathrm{~cm}$ and $\ell=4.85 \mathrm{~cm}$. The transit time of the light through the short fiber section inside the cell is $\tau_{t}=0.24 \mathrm{~ns}$, producing an envelope function with $3 \mathrm{~dB}$ bandwidh and cutoff freguency of $1.87 \mathrm{GHz}$ and $4.2 \mathrm{GHz}$. The resonance peaks in Fig. $7-6 \mathrm{~b}$ are plotted only to approximately $400 \mathrm{MHz}$. Unfortonately, RF interference in the measurement instrumentation (peculiar to this experimental configuration only) precluoded data recotding to nearer the $3 \mathrm{~dB}$ bandwidth of the envelope function.

\section{Rectangular Coils}

Fiber current sensors coils with rectangular cross sections (though not practical to implement because of the shap corners) are excellent for the theoretical study of high frequency effecss in the Faraday response. This particular geometry is chosen for modeling because (1) it represents a coil cross section that is noncircular in alt respects, thus exhibiting several unique high frequency features; and (2) it lends itself to a simple analytical description for predicting centered, noncentered and highly elongated coil frequency responses. Ideally, a model of an elliptically-shaped coil is desired to represent a realistic fiber current sensor coil that might have a displaced conductor or a compressed cross section; however, the mathematical treatment is quite complicated, and the resulting model begins to break down as the coil becomes very eccentric. The rectangular coil model provides a good compromise for this study.

The Faraday response model is constructed using the geometry shown in Fig. 7-7. Expressions similar to Eq. (7-21) are used to determine the rotation in each segment of 
the reciangular cross section. The rectangle has dimensions $2 \mathrm{w}$ (width) by $2 \mathrm{~h}$ (height). Light enters the coil at the location marked by $t_{0}$ in Fig. 7-7. One complete loop around the rectangle requires light travel through five fiber segments; the total number of fiber segments in the coil is then $5 \mathrm{~N}$. The Faradiay rotation in the first segment is

$$
\Theta_{1}(\omega)=V_{0}\left(\frac{c}{n}\right)\left(w+X_{1}\right) \int_{m \tau_{1}}^{m \tau_{1}+t_{1}} \frac{e^{-j \omega t}}{\left(w+X_{1}\right)^{2}+\left(\frac{c}{n}\right)^{2}\left(m \tau_{1}-t\right)^{2}} d t,(7-23)
$$

where $\mathrm{m}$ is the turn counter, 0 to $\mathrm{N}-1, \tau_{1}=2(w+h)(n / \mathrm{c})$ is the light transit time through one complete fiber loop. and $t_{1}=h(n / c)$. In the second segment (top horizontal).

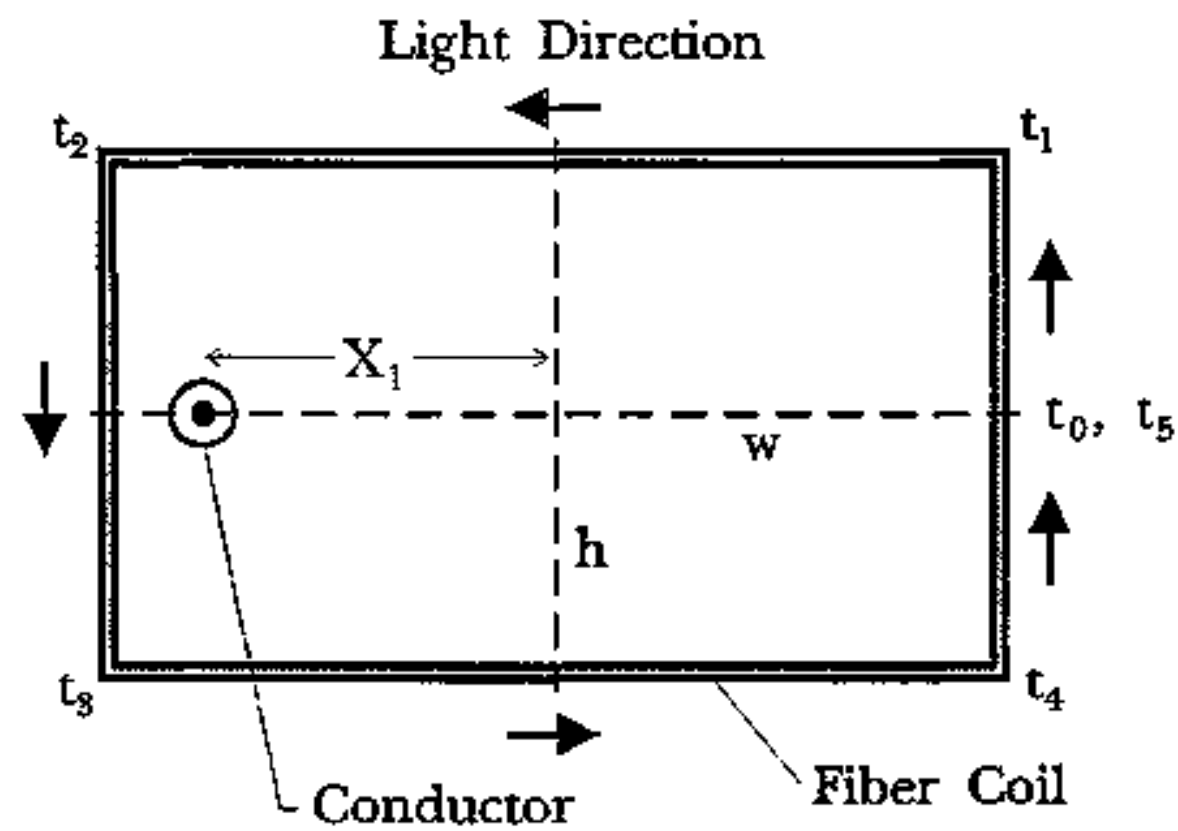

Fig. 7.7, The geometrical layout for the rectangular coil model. The conductor is displaced in the coil cross section, and the coil shape is allowed to vary. 


$$
\Theta_{2}(\omega)=V B_{0}\left(\frac{c}{n}\right) h \int_{m t_{1}+t_{1}}^{m \tau_{1}+t_{2}} \frac{e^{-j \omega t}}{h^{2}+\left(\frac{c}{n}\right)^{2}\left[m \tau_{1}+t_{1}+\frac{n}{c}\left(w+x_{1}\right)-t\right]^{2}} d t \text {, }
$$

where $t_{2}=t_{1}+2 w(n / c)$. The third segment (left vertical) prodnces a rotation given by

$$
\theta_{3}(\omega)=\operatorname{VB}_{0}\left(\frac{c}{n}\right)\left(w-x_{1}\right) \int_{m \tau_{1}+t_{2}}^{m t_{1}+t_{3}} \frac{e^{-j \omega t}}{\left(w-x_{1}\right)^{2}+\left(\frac{c}{n}\right)^{2}\left[m \tau_{1}+t_{2}+\frac{n}{c} h-t\right]^{2}} d t .
$$

where $t_{3}=t_{2}+2 t_{1}$. An expression for $\theta_{4}(\omega)$ for the lower horizontal segment is similar to Eq. (7-24) with appropriate changes in the time; and the final rectangular segment has a rotalion, $\Theta_{s}(\omega)$, similar to Eq. (7-23). The total response is computed by summing the individual rotations, then normalizing to the zero frequency rotation.

$$
\frac{\theta(\omega)}{\theta_{0}}=\frac{\sum_{m=0}^{N-1}\left[\theta_{1}(\omega)+\theta_{2}(\omega)+\theta_{3}(\omega)+\theta_{4}(\omega)+\theta_{5}(\omega)\right]}{2 N V B_{0}\left[\tan ^{-1}\left(\frac{h}{w+X_{1}}\right)+\tan ^{-1}\left(\frac{h}{w-X_{1}}\right)+\tan ^{-1}\left(\frac{w+X_{1}}{h}\right)+\tan ^{-1}\left(\frac{w-X_{1}}{h}\right)\right]}
$$

Equation (7-26) is evaluated numerically to determine the total Faraday rotation. Throughout the computations, the rectangle geometries will vary, but the perimeter of the rectangle is held constant so that total transit time, $\tau$, is the same as that in an equivalent circular coil. The light entry point into the fiber can also be varied by letting $\mathrm{X}_{1}>0$ to simulate $\xi=0.5$ and setting $\mathrm{X}_{1}<0$ for $\xi=0$.

The frequency response of the Faraday rotation for the rectangular coil models are plotted in Fig. 7.8. In the models leading to these two plots, the conductor is allowed 


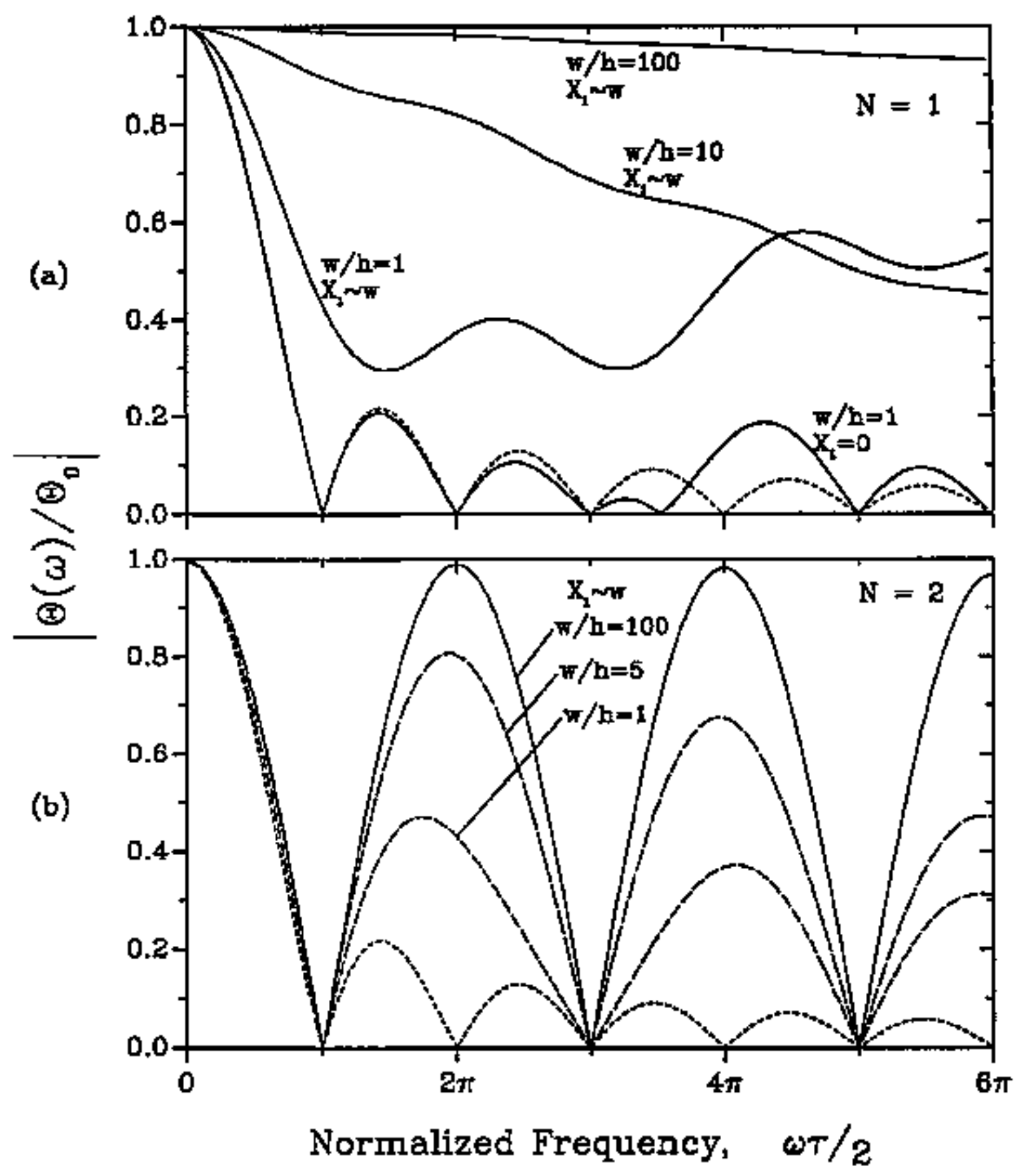

Fig. 7-8. Model predictions for a Faraday effect fiber current sensor coil with a varying rectangular cross section. (a) A single turn coil with the conductor centered in a sguare cross section and with the conductor displaced to the coil edge in a direction away from the light entry point. The rectangular cross section is varied with the conductor displaced. (b) A two turn coil with the conductor displaced to the coil edge. The dotled curve in each plot is the reference for an equivalent centered, circular coi!. 
to move toward the fiber coil $\left(x_{1}>0\right)$ and the rectangular cross section is varied. The dotted curves in both Figs. 7.8a and 7.8b are the response for a centered circular coil which has a circumference equal to the perimeter, $4(w+h)$. In Fig. $7-8 \mathrm{a}$, the response for a single tum square coil (width/height $=1$ ) is shown. This response has identical main Iobe bandwidth and cutoff frequency as that for the centered, circular coil. However, a noticeable deviation in the curves occors near $\omega \tau / 2=4 \pi$. This is due to the four-fold symmetry created by the four identical coil sides. As might be expected, the exact shape of this small response enhancement near $\omega \pi / 2=4 \pi$ will depend on the choice of light entry point in the square coil. Also shown in Fig. 7-8a is the effect of displacing the conductor to the edge of the square coil $\left(w / h=1, X_{1} \sim w\right)$ in a direction away from the light entry point (equivalent to $X_{1} / R-1$ and $\xi=0.5$ in the circular coil). This curve shows an increased bandwidth in the low frequency lobe and higher frequency oscillations that result from both two-fold and four-fold symmetries. This corve can be compared directly to its counterpart for the circular coil in Fig. 6-2a. Noted similarities are the lack of response nulls at the higher frequencies and rejative response magnitudes near $1 / 2$.

With the conductor fully displaced, the rectangular coil cross section is compressed to a highly elongated state. Response curves are shown in Fig. 7.8a for an intermediate compression state $(\mathrm{w} / \mathrm{h}=10)$ and near total compression $(\mathrm{w} / \mathrm{h}=100)$. The frequency response for the coil with large eccentricity and near-maximurn condvctor displacement approaches that of the delta function model for a single tom coil. This response exhibits a near-infinite bandwidth.

In Fig. 7-8b the Faraday response is shown for a two-ton coil, again with the conductor displaced almost fuily to the edge of the coil; the cross section of the rectangle is allowed to vary in shape. For the square coil, resonant responses are observed near the frequencies defined by $\omega \tau / 2=2 \mathrm{~m}^{\prime} \pi$. This resonant behavior is very similar to that of the two-turn circular coil with displaced conductor. The relative magnitude of the first 
higher frequency resonance peak for the square coil in Fig. $7.8 \mathrm{~b}$ is near $1 / 2$, and the magnitudes of subseguent higher frequency peaks diminish. If the model were to atlow the limiting case of $X_{1}=w$ without producing a singularity, the magnitude of all the higher frequency resonances would be $1 / 2$ as demonstrated for the circular coil in Chapter 6. As the cross section of the rectangular coil is compressed, the relative magnitude of the resonance peaks in Fig. $7-8 \mathrm{~b}$ increases. When $w / \mathrm{h}=100$, the predicted Faraday response approaches that of the delta function model shown in Fig. 7-2 for $\mathrm{N}=2$. The bandwidth increase in the main lobe is noted, and the magnitudes of the higher frequency resonances are very near one.

The curves in Fig. 7.8 are presented to help visualize the frequency responses for coils that are in intermediate states between a symmetric (i.e., circular) coil with fully displaced conductor and a highly elongated coil with fully displaced conduclor. During the transition of coil cross section between these two states, the magnitude of the resonance response peaks (see Fig. 7-8b) progresses from approximately $1 / 2$ to very near unity. It is expected that coils that initially have a circular cross section will exhibit similar high frequency responses as the cross section is compressed.

Many general high frequency characteristics of fiber current sensors can be investigaled theoreticalty using the rectangular coil model. One good example is a single twm coil with the conductor placed at the center and the coil cross section compressed. Modeling the initial shape as a square, the frequency response begins as that seen in Fig. $7-8 \mathrm{a}$ for the curve with $w / h=1$ and $X_{1}=0$. After compression, the response is much like the curve in Fig. 7-8b for $w / h=100$. The current sensor response will resemble that of a "double delta function", since in the highly elongated state, the light in the fiber makes two close approaches to the conductor for a single coil turn. The frequency spacing between resonances is determined from the transit time of light through half a coil turn, $\tau_{1} / 2$, resulting in $\omega_{\mathrm{R}} \tau / 2=2 \mathrm{~m}^{\prime} \pi$, which is exactly that shown in Fig. $7-8 \mathrm{~b}$ for the two-turn coil. 
The resonance phenomenon just described is observable in the frequency response of almost any fiber coil with arbitrary cross section. If the conductor is centered in the coil cross section, then the sensor main lobe bandwidth and frequency response will closely approximate that of the centered, circular coil. Deviations in the higher frequency response will result from symmetries pecultar to the chosen coil geometry and the choice of the light entry point. Then in the highly elongated state, the response will approach that predicted by the delta function model. Also, it is easily envisioned that specific features in the responses for coils with displaced conductors in arbitrary cross sections can be predicted using information already generated from the delta function and rectangulaf coil models. In general, it appears that predicting the Faraday frequency response for many coil geometries is possible through use of the framework developed here for the noncircular current sensor coils. 


\section{CHAPTER 8}

\section{TRAVELLING WAVE FIBER CURRENT SENSORS:}

\section{BASIC CONCEPTS AND THEORY}

Manipulation of the fiber cerrent sensor coil cross section and the position of the conductor relative to the coil changes the higher frequency characteristics of the Faraday response, but has limited effects on the sensor bandwidth, especially for multiturn coils. Bandwidth can be enhanced in the transit time limited fiber sensors by deviating from the planar coil structure and expanding the fiber into a helix. In this geometry the electromagnetic wave propagating along the conductor experiences partial phase matching with the light propagating in the fiber. This travelling wave configuration entances the Faraday rotation at the higher freguencies, and thereby increases the bandwidth.

The travelling wave concept has been used extensively in electro-optic modulators [62], and integrated optic devices with bandwidths well beyond $10 \mathrm{GHz}$ have been fabricated [66]. In these modulators, however, the Pockel's effect (inlcraction between the light and the electric field) is used, and the two interacting signals propagate codirectionally and in the same dielectric medium. In a fiber current sensor, the magnetic field is generally orthogonal to the direction of current propagation, while the Faraday rotation is dictated by $\mathbf{B}$ * $\mathrm{dl}$ and is maximized when the field and light are codirectional. Implementing a Faraday effect travelling wave concept is thus much more difficult since both $\mathbf{B}$ and $\mathbf{k}$ cannot simultaneously be directional with the light. The helical coil geometry allows for partial phase matching, but further enhancement of the Faraday signal requires slowing the propagation of the electromagnetic wave.

\section{Travelling Wave Fiber Current Sensor Model}

A basic travelling wave fiber current sensor is shown schematically in Fig. 8-1. 
The helical singlemode fiber coil is immersed in the dielectric of a coaxial transmission line. If the frequency of operation is limited to a range where only a TEM mode exists in the transmission line and the fiber coil is centered about the coax center conductor, then Eq. (3-9) describes the Faraday response for the system. It is possible for the coil to assume any position relative to the center conductor, which then requires use of Eq. (3-7) to fully describe the frequency dependent Faraday interaction. This added complexity is addressed briefly in Chapter 12; however, for most of this study of travelling wave fiber current sensors, the coil is taken to be coaxial with the conductor. Two further simplifications are introduced into the model: the fiber helix consists of $\mathrm{N}$ complete tams with a constant pitch, $x_{\theta}$ and the coax line dielectric is free of magnetic materials, $\mu_{r}=1$. Using these assumptions, the angle $\psi$ is no longer time dependent,

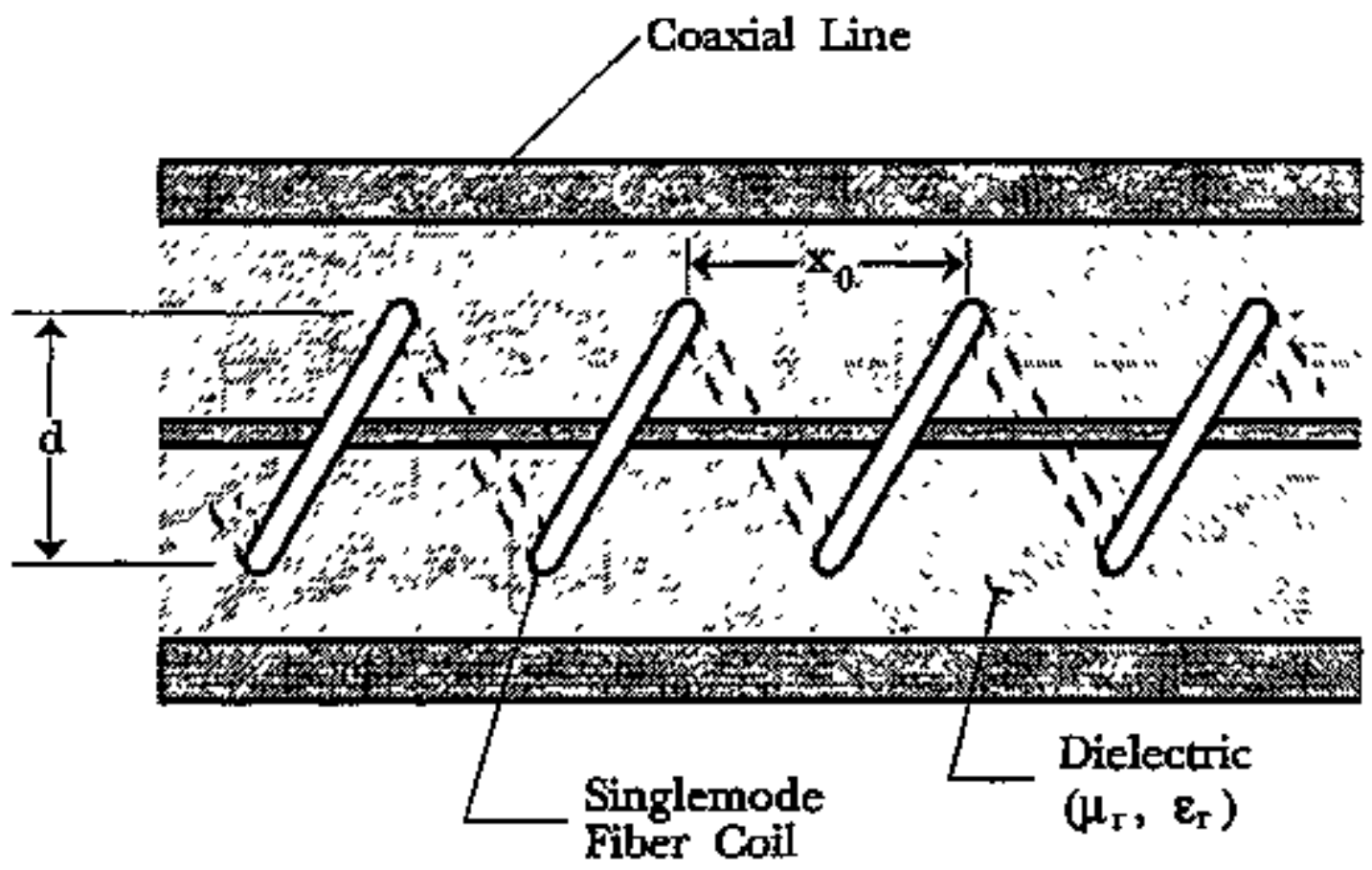

Fig. 8-1. A travelling wave fiber current sensor constructed of a helical singtemode fiber coil immersed in the dielectric of a coaxial transmission line. 


$$
\cos \psi=\frac{\mathrm{Nx}_{0}}{\mathrm{~L}},
$$

and

$$
\sin \psi=\frac{\mathrm{N} \pi \mathrm{d}}{\mathrm{L}},
$$

where $L$ is the total fiber length in the coil helix and $d$ is the mean diameter of the coil measured radiafly from the conductor axts. Substituting Eqs. (8-1) into Eq. (3-9) results in a general expression for the Faraday rotation of the simplified travelling wave sensor:

$$
\Theta(\omega)=\theta_{0} \frac{1}{\tau} \int_{0}^{\tau} e^{-j \omega t\left[1-\frac{\sqrt{\varepsilon_{L}}}{\eta} \frac{N_{\Sigma_{s}}}{L}\right]} d t .
$$

In Eq. $(8-2) \tau=(n / c) 2 \pi R N / \sin \psi$, which differs from that for the planar coil by $(\sin \psi)^{4}$. This factor arises because the helical coil reguires a longer fiber length for a given coil dianeter, $d$. The actual length of the fiber is given by

$$
L=N \sqrt{(\pi \mathrm{d})^{2}+x_{0}^{2}},
$$

Which when substituted into Eq. (8-2) results in a final integral expression for the Faraday response:

$$
\theta(\omega)=\theta_{0} \frac{1}{\tau} \int_{0}^{\tau} e^{-j \omega t\left[1-\frac{\sqrt{t_{1}}}{n \tau t \sqrt{\left.(d /)^{2}\right)^{2}+1 / r^{2}}}\right]} d t .
$$

If the material relative permittivity is considered constant (independent of 
frequency), Eq. (8-4) can be solved in manner similar to Eq. (5-1) for the centered, circular (and planar) coil. The solution becomes

$$
\theta(\omega)=\theta_{0}\left[\frac{\sin (\zeta)}{\zeta}\right] \mathrm{e}^{-\mathrm{j} \zeta}
$$

where

$$
\zeta=\frac{\omega \tau}{2}\left(1-\frac{\sqrt{\varepsilon_{\mathrm{r}}}}{\mathrm{n} \pi \sqrt{\left(\mathrm{d} / \mathrm{x}_{0}\right)^{2}+1 / \pi^{2}}}\right) .
$$

At the low frequencies, $\omega \rightarrow 0, \zeta$ becomes zero and Eq. (8-5a) reduces to Eq. (2-3) as expected. If the coil pitch, $x_{0}$ becomes small, the contribution from the second term in the parenthesis of Eq. (8-5b) is diminished. If $\mathrm{x}_{0}=0$, then Eq. (8-5a) is identical to Eq. (5-3) for the planar coil. The relative magnitude of the Faraday response in Egs. (8-5) has a form simitar to Eq. (5-4):

$$
\left|\frac{\Theta(\omega)}{\Theta_{0}}\right|=\left|\frac{\sin (\zeta)}{\zeta}\right| .
$$

The $3 \mathrm{~dB}$ bandwidth occurs when $\zeta \approx \sqrt{2}$ which leads to [60]

$$
\Delta \mathrm{B}=\frac{\sqrt{2} \mathrm{c}}{\pi^{2} \mathrm{nN} \mathrm{x}_{0}}\left[\sqrt{\left(\delta / \mathrm{x}_{0}\right)^{2}+\frac{1}{\pi^{2}}}-\frac{\sqrt{\varepsilon_{\mathrm{r}}}}{\mathrm{n} \pi}\right]^{-1} .
$$

The travelling wave sensor bandwidth now shows a dependence on the coaxial line relative permittivity and the fiber coil diameter-to-pitch ratio, $d / x_{0}$.

The bracketed factor in Eq. (8-7) can be equal to zero, which theoretically gives 
an infinite bandwidth. This condition arises from perfect phase matching between the two interacting electromagnetic signals. When Eq. (8-6) for the travelling wave coil configuration is conpared to Eq. (5-4) for the planar coil geometry (the baseline configuration), an enhancement factor equivalent to

$$
F=\frac{1}{1-\frac{v_{1}}{v_{p b}}} \text {, }
$$

is extracted [62]. The travelling wave enhancement is deternined by the relative phasing between the light in the fiber, $v_{f}=(c / n) \cos w$ (in the direction of $k$ ) and the vetocity of the

TEM wave, $v_{\mathrm{ph}}$, as given by Eq. (3-6). When the telative phase velocilies are identical, the enhancernent is infinite.

The relative magnitude of the Faraday rolation from Eq. (8-6) is plotted versus the nomalized frequency, $\omega \tau / 2$, in Fig. 8-2. Also plotted is a reference curve taken from Eq. (5-4). In the example in Fig. 8-2, a travelling wave enhancement of slightly larger than two is used for illustration.

\section{The Bandwidth Enhancement Factor}

A bandwidth enlancement factor (BEF) is determined by the ratio of the travelling wave sensor bandwidth to the planar coil bandwidth:

$$
\mathrm{BEF} \equiv \frac{\Delta \mathrm{B} \text { (travelling wave) }}{\Delta \mathbf{B} \text { (planar) }}
$$

Upon substitution of Eqs. (8-7) and (5-8) [60], 


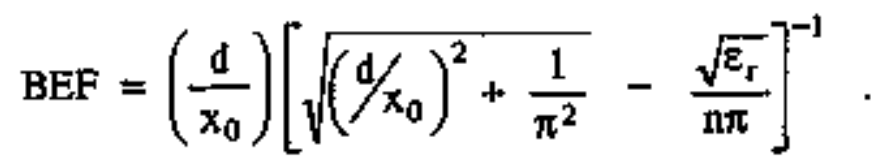

In defining the $\mathrm{BEF}$, the number of fiber turns and the coil diameter remsin fixed for the two coils being compared. This means the low frequency Faraday sensitivity is unchanged from that described by Eq. (2-3). Thus, the BEF can be used as a figure of merit to give a quantifiable eslimate of sensor performance improvement for traveling wave configurations. The BEF can also be used directly to compute the increase in the sensitivity-bandwidth product over that for the planar coil given in Eq. (5-10).

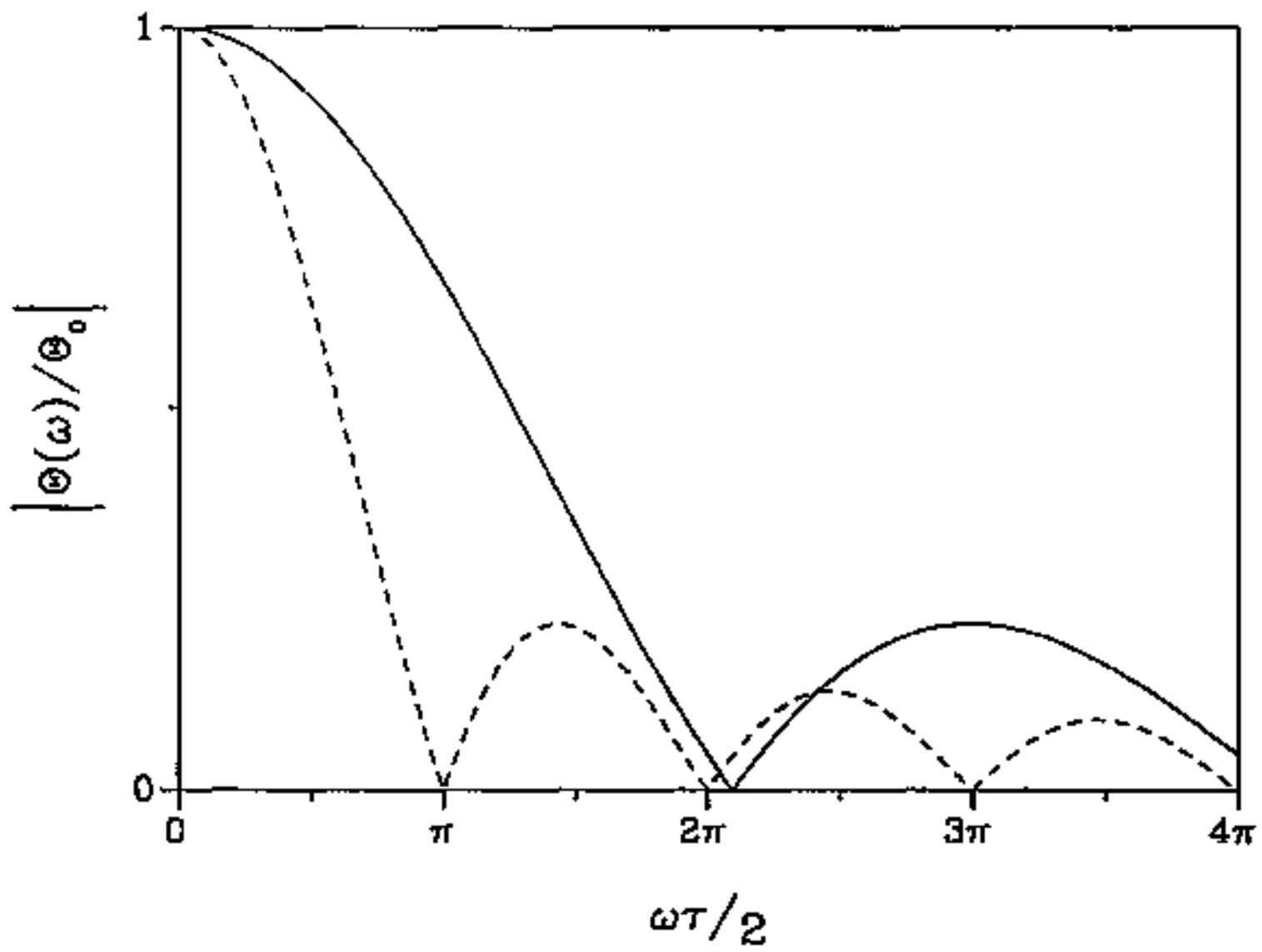

Fig. 8-2. A typical Faraday response curve for a travelling wave fiber current sensor. The dotted reference curve is the response for an equivalent sensor coil with coplanar turns. 
Equation (8-10) is very similar to the enhancement factor given by Eq. (8-8) when the respective velocities are substituted. The only difference is the added fiber length,

$$
\Delta \mathrm{L}=\sqrt{1+\left(\mathrm{x}_{0} / \mathrm{d \textrm {d }}\right)^{2}}
$$

that must be used in the helical coil in order to maintain a fixed diameter. Equation (8-8) is a generic expression for any travelling wave structure that accounts only for the relative phase velocity mismatches; Eq. (8-10) is specific to this fiber configuration.

The BEF is strongly dependent on the diameter-to-pitch ratio of the travelling wave fiber sensor coil and the rejalive permitivity of the slow-wave structure (the coaxial line). Figure 8-3 is a plol of the BEF, Eq. (8-10), for several values of $\varepsilon$, using $\mathrm{d} / \mathrm{x}_{0}$ as the independent variable. The fiber refractive index is assumed to have a nominal value of 1.46. For any $\varepsilon_{\mathrm{r}}$ a value of $d / x_{0}$ exists for which a maximum BEF occurs. If $\varepsilon_{r}>n^{2}$, the maximum BEF approaches infinity. This complete phase matching condition (the travelling wave condition) occurs when the bracketed factor in Eq. (8-10) equals zero, or when

$$
\frac{\mathrm{d}}{\mathrm{x}_{0}}=\frac{1}{\pi} \sqrt{\frac{\varepsilon_{\mathrm{t}}}{\mathrm{n}^{2}}-1} \text {. }
$$

In Fig. 8-4, the values of $\varepsilon_{\mathrm{r}}$ and $\mathrm{d} / \mathrm{x}_{\mathrm{0}}$ are ploted for which the travelling wave condition, Eq. (8-I2) are met. It is obvious from Fig. 8-4 that slow-wave structures with larger relative permittivities are needed if the fiber hejix has a small pitch.

In Fig. 8-3, if $\varepsilon_{\mathrm{r}}<n^{2}$, the maximum BEF remains finite. For $\varepsilon_{\mathrm{r}}=2$, the BEF attains a peak value of $-4.0 \mathrm{at} d / \mathrm{x}_{0}=0.083$; and for $\varepsilon_{\mathrm{q}}=1$, an air or vacuum dielectric, the BEF peaks at 1.37 when $d / x_{0}=0.33$. Thus, merely expanding the fiber coil into a 


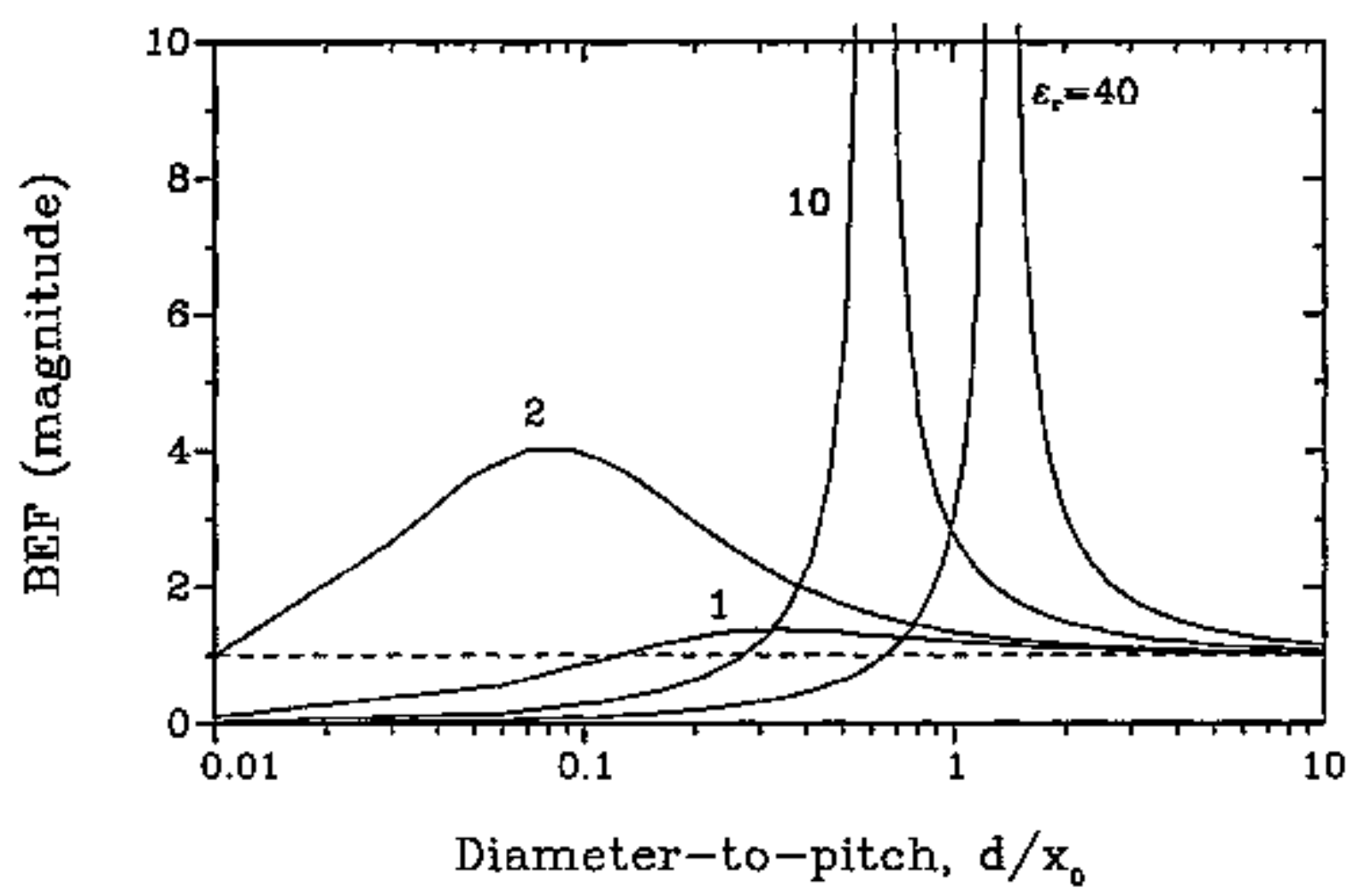

Fig. 8-3. The magnitude of the bandwidth enhancentent factor as a function of the travelling wave coil diameter-to-pitch ratio. Curves are plotted for a wide range of relative permistivity values.

helix does not provide much improvement in the sensor bandwidth. The propagation of the magnetic wave must also be slowed, preferably by using a dielectric with a large relative permittivity.

Two other features are noted in the curves in Fig. 8-3. As $d / x_{0} \rightarrow \infty$, or the coil pitch becomes small, the BEF approaches one for all values of $\varepsilon_{q}$ (no enhancement occurs as expected). But as $d / x_{0} \rightarrow 0\left(x_{0} \rightarrow \infty\right.$, infinite pitch), the BEF approaches zero for all

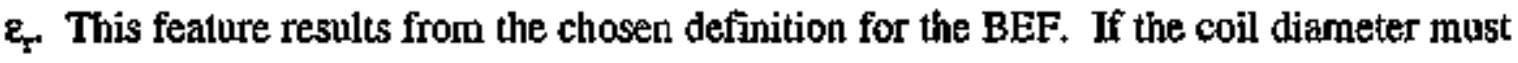
remain constant, then as $x_{0}$ increases so does the total fiber length according to Eq. (8-3). Eventually, the transit time of the light through this fitber lenglh becomes prohibitively long and the bandwidth for the sensor decreases to zero. In order to keep the travelling 


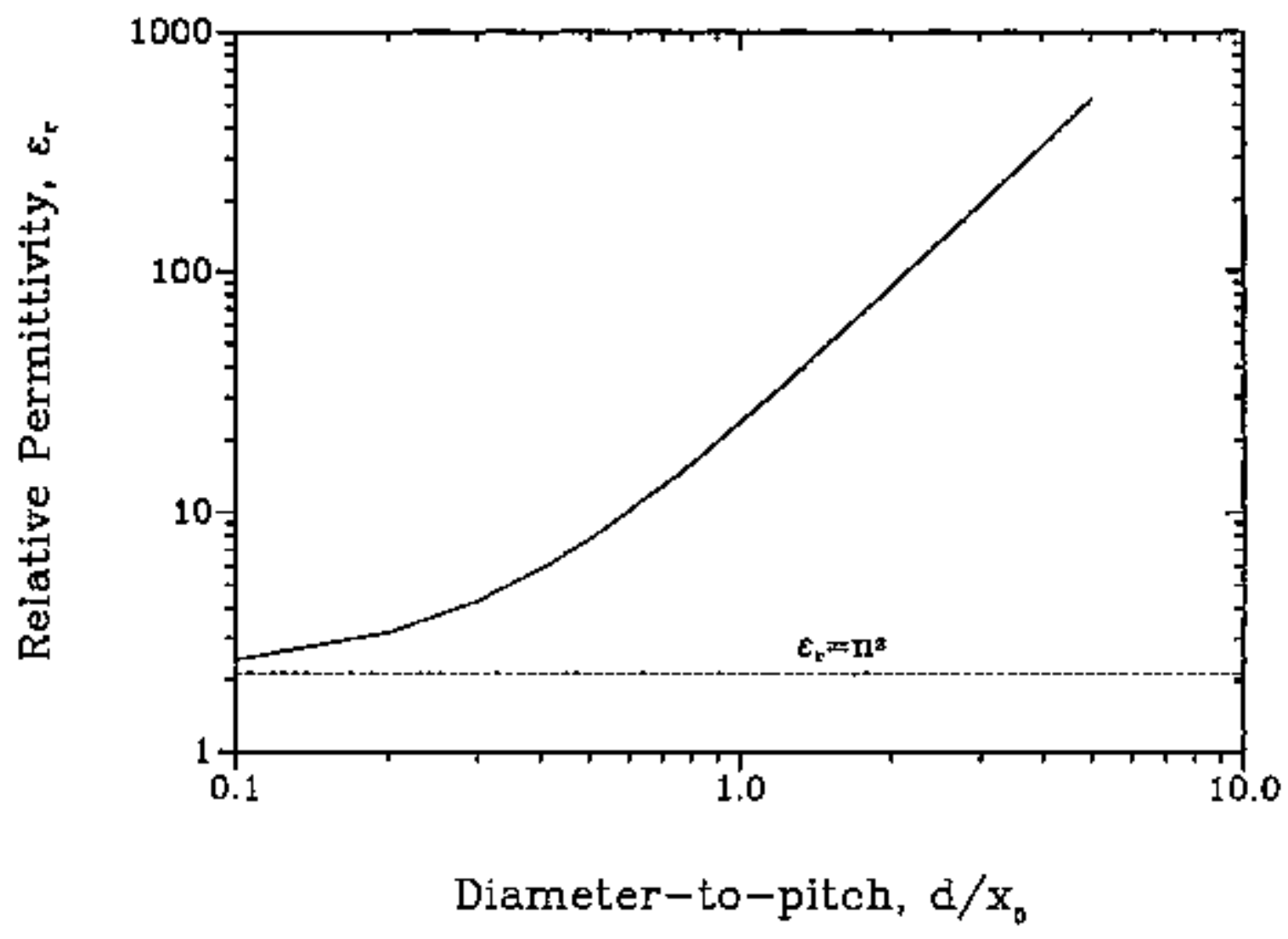

Fig. 8-4. A plot of the relative permittivity of the dielectric medium required for maximum Faraday response bandwidth versus the sensing fiber diameter-to-pitch ratio. For the ideal travelling wave sensor with $\varepsilon_{\mathrm{r}}>\mathrm{n}^{2}$, the theoretical maxinum bandwidth approaches infinity.

wave sensor at a practical length and yet produce as large a BEF as possible, emphasis is again placed on using the high relative pernittivity materials. 


\section{CHAPTER 9}

\section{TRA VELLING WAVE TRANSMISSION CELLS}

The travelling wave fiber current sensor shown in Fig. 8-1 was implemented using coaxial transmission cells with helical fiber coils mounted in the cell working volume [34]. Two basic cell configurations were used: one with an air dielectric, the other with liquid dielectrics of varying relative permittivity. The airline provided a baseline measurement for Faraday current sensor response when only a helically-shaped coil was used. Liquids were chosen as coaxial line dielectrics for the second configuration because they have large $\varepsilon_{r}$, they are readily available in a wide range of permittivities, and they can easily assume the shape of the cell interior and fill the large volumes required.

The fiber coils were constructed from low birefringence singlemode fiber with an 80 um cladding diameter. Fibers were twisted at a rate of -30 turns/meter and wound on $3 \mathrm{~cm}$ diameter mandrels. The selected twist rate was adequate for the circular birefringence bias to overcome the bend-induced birefringence at the chosen coil diameter. Faraday responses then could be measured directly from relative changes in the light intensity as described in Chapter 4 . The coil diameter was held constant for all test designs, but the pitch of the helical coil varied between 1.5 and $3.0 \mathrm{~cm}$; several values of $d / x_{0}$ were used. In some configorations, the coils were quite long; however, the total number of tums was limited so that coils never exceeded $45 \mathrm{~cm}$ in axial length. The number of coil turns varied from 10 to 20 .

A single coaxial cell outer structure was used for all travelling wave experiments. The outer conductor diameter was $5.75 \mathrm{~cm}$. In order to maintain a $50 \mathrm{Q}$ nominal impedance on the transmission line, the inner conductor diameter varied according to Eq. (A-11) for the appropriate value of $E_{r}$ Properties of the travelling wave transnission cells - SWR, impedance and transmission magnitzode - were determined using ANA 
measurements and Eqs. (A-25) and (A-26). These properties were important in assessing the useful operating frequency range for the liguid cells and necessary in developing accurate response models that accounted for atl the liquid parameters.

The transmission cells were positioned as shown in the experimental arrangement of Fig. 4-1. A photograph of the bench-top hardware for this configuration is shown in Fig. 9-1. All remaining instrumentation and procedures for the measurements of the travelling wave current sensor response were as ontlined in Chapter 4.

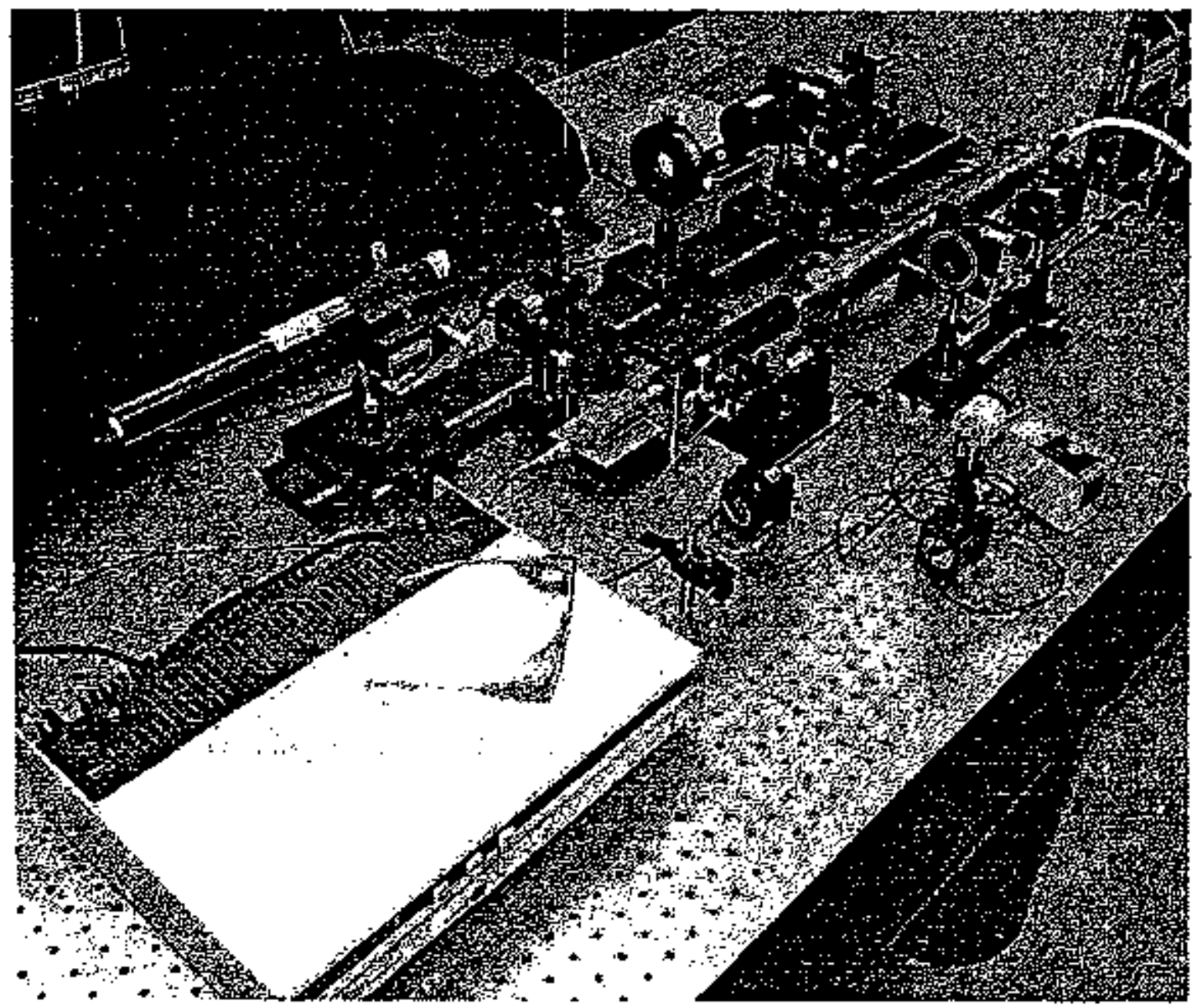

Fig. 9-1. A bench-top photograph of the experimental hardware used during testing of the travelling wave fiber current sensors. 


\section{Alriline Cell}

The coaxial airline travelling wave test cell is described as Cell \#2 in Table A-1 of Appendix A. The cell center conductor diameter is $2,49 \mathrm{~cm}$ (for $50 \Omega$ characteristic impedance), and the working volume length is $45.7 \mathrm{~cm}$. The fiber sensor coil consisted of 10 turns wound on a polyurethane foam mandrel of $3.0 \mathrm{~cm}$ diameter. This coil support then fit directly over the cell center conductor. A view of the disassembled airline is shown in the photograph, Fig. 9-2. Tapered end sections of both inner and outer conductors can be seen at the left of the photo. The ends of the fiber coil are secured to the foam mandrel by first sewing several thread loops around the fiber and through the foam; then a dab of UV-coring epoxy is hardened at the fiber-thread interface. The twist is locked into the fiber turns, and the coil leads are allowed to dangle (untwisted) so they may be fed through the wall of the cell outer conductor section.

The electromagnetic performance characteristics of the airline travelling wave cell were quite good. SWR and impedance magnitude of the cell are plotted versus freguency in Fig. 9-3. The cell SWR never exceeds 1.23 and the impedance varies

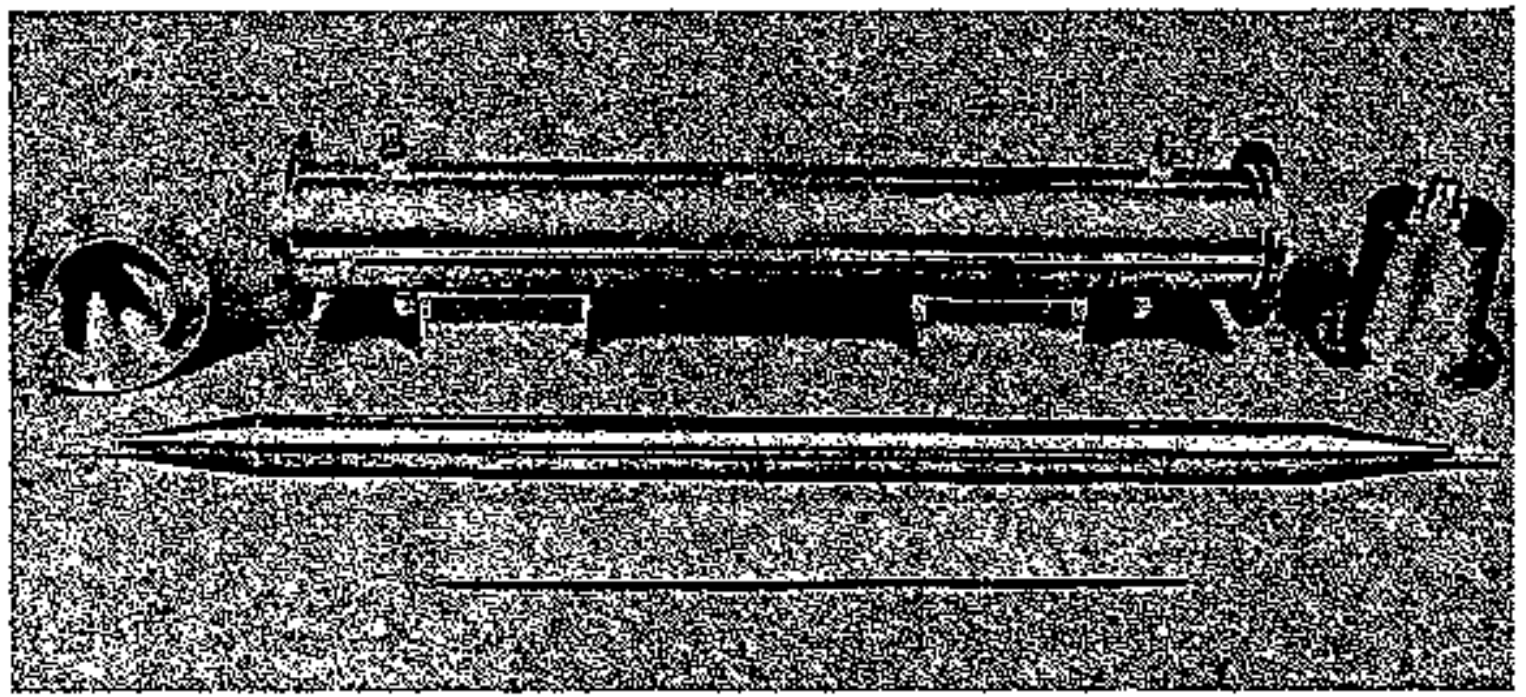

Fig. 9-2. Photograph of the disassembled travelling wave airline cell. A 10-tum, helical Fiber coil on a foam mandrel is located at the bottom of the photo. 

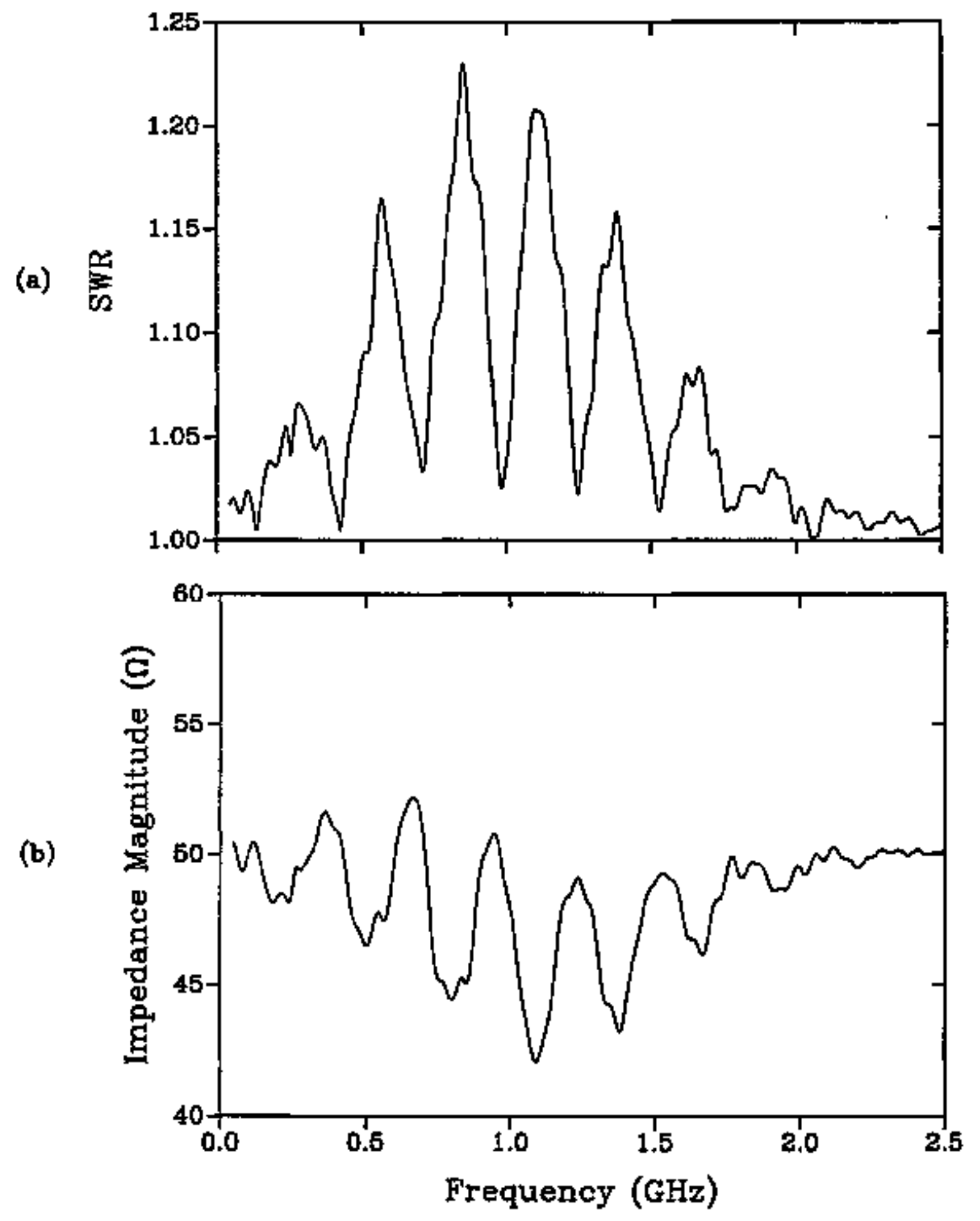

Fig. 9-3. The measured SWR (a) and the impedance magnitude (b) of the travelling wave airline cell with the optical fiber sensor inside. 
between 42.5 and $52 \Omega$. Frequency spacing between the larger oscillations in both the SWR and the impedance is approximately $260 \mathrm{MHz}$. This conesponds to resonant reflections at the transitions from the RF connectors into the cell air space. The fiber coil and foam mandrel do not create noticeable perturbations in the electromagnetic characteristics. The useful operating frequency range of the airline cell extends beyond $2.3 \mathrm{GHz}$, which is the cutoff frequency for cell higher order modes. Such a high test frequency, however, is not needed since the fiber current sensor Faraday response is limited to much lower values.

\section{Liquid Cells}

Many organic and inorganic liguids with large relative permittivities $[67], \varepsilon_{\mathrm{r}}>10$, were considered during design of the travelling wave sensors. Three -1 -propanol, methanol, and water - were chosen for testing because they spanned a wide range of $\varepsilon_{r}$ values and were relatively nonhazardous in the small volumes used. The static or low Irequency relative permitivity of these three liquids is $20.1,32.63$, and 78.54 , respectively, at $25^{\circ} \mathrm{C}$. One disadvantage of using liguid dielectrics in the travelling wave cell is the temperature dependence of $\varepsilon_{\mathrm{r}}$ Selected values of the relative permittivity for the three test liquids are given in Tables 9-1 and 9-2. Near ambient temperature in the laboratory, values for $\varepsilon_{\mathrm{r}}$ vary only slightly. Thus, no temperature control was deemed necessary, but a monitor was provided to indicate liquid temperature. This sensor, a Fluoroptic ${ }^{\text {TM }}$ thermometer [68) from Luxtron, is seen in Fig. 9-1.

Figure $9-4$ is a close-up view of the futly assembled liquid travelling wave cell. (This cell is also seen in the experimental setup of Fig. 9-1.) Inlet and vent ports for liquid filling are visible on the top of the cell. Exit holes for the fiber sensor required sealing to prevent liguid leakage. The optical fiber test coil is supported in the liquid medium by a teflon tube. A helical groove with a semicircular cross section was machined in the outside surface of the tube as a guide for the twisted fiber. The fiber 
Table 9-1.

Relative Permittivity of the Test Liquids at Selected Temperatures [67].

$\begin{array}{cccc}\text { Temperature }\left({ }^{\circ} \mathrm{C}\right) & \text { 1-Propanol } & \text { Methanol } & \text { Water } \\ -113 & - & 64.0 & - \\ -80 & 38.0 & 54.0 & - \\ -34 & 29.0 & - & - \\ -20 & - & 40.0 & - \\ 20 & - & 33.62 & 80.37 \\ 25 & 20.1 & 32.63 & 78.54 \\ 200 & - & - & 34.5\end{array}$

was anchored to the mandrel using the same thread-and-epoxy technique as used for the airline coil. Several mandrel tubes were used, each with a different helical pilch. The teflon mandrel was aligned coaxjally in the cell using several teflon support rings. These rings are seen in the end-view photograph of the opened liquid cell in Fig. 9-5a. The support rings asso aligned the center conductor coaxially in the cell. Because of the large relalive permittivity of the liquid, the center conductor diameler required to maintain a $50 \Omega$ characteristic impedance in the cell was often very small. Thus, external support was needed.

Barely visible in Fig. 9-5a is the optical fiber spooling off the end of the teflon mandrel and exiting the cell. This section of fiber was untwisted, and care was taken to nol stress it during cell assembly. Figure 9-5b shows the disassembled travelling wave cell. The 0 -rings used to seal the cell onter conductor components are vișible on the two endcap sections. Also, short teflon extension tubes are seen in the endcaps; these help 


\begin{tabular}{|c|c|c|}
\hline & Relative Permittivity of Water. & \\
\hline Temperature $\left({ }^{\circ} \mathrm{C}\right)$ & $\varepsilon$ [Ref. 69] & $\varepsilon$ [Ref. 70] \\
\hline 0 & 87.74 & 87.90 \\
\hline 5 & 85.76 & 85.90 \\
\hline 10 & 83.83 & 83.95 \\
\hline 15 & 81.95 & 82.04 \\
\hline 20 & 80.10 & 80.18 \\
\hline 25 & 78.30 & 78.36 \\
\hline 30 & 76.55 & 76.58 \\
\hline 35 & 74.83 & 74.85 \\
\hline 40 & 73.15 & 73.15 \\
\hline 45 & 71.51 & 71.50 \\
\hline 50 & 69.91 & 69.88 \\
\hline 55 & 68.34 & 68.30 \\
\hline 60 & 66.81 & 66.76 \\
\hline 65 & 65.32 & 65.25 \\
\hline 70 & 63.86 & 63.78 \\
\hline 75 & 62.43 & 62.34 \\
\hline 80 & 61.03 & 60.93 \\
\hline 85 & 59.66 & 59.55 \\
\hline 90 & 58.32 & 58.20 \\
\hline 95 & 57.01 & 56.88 \\
\hline 100 & 55.72 & 55.58 \\
\hline
\end{tabular}


maintain radial dielectric continusty throughout the entire length of the cell interior.

The presence of the teflon support structures inside the cells often created numerous points for reflection of the electromagnetic waves. Tefion was chosen as a suppont material because it is impervious to all tiquids considered as potential dielectric materials. However, the teflon has a relative permittivity of 2.0 , which differs significantly from that of any of the liquids. Impedance mismatches conld occur at many

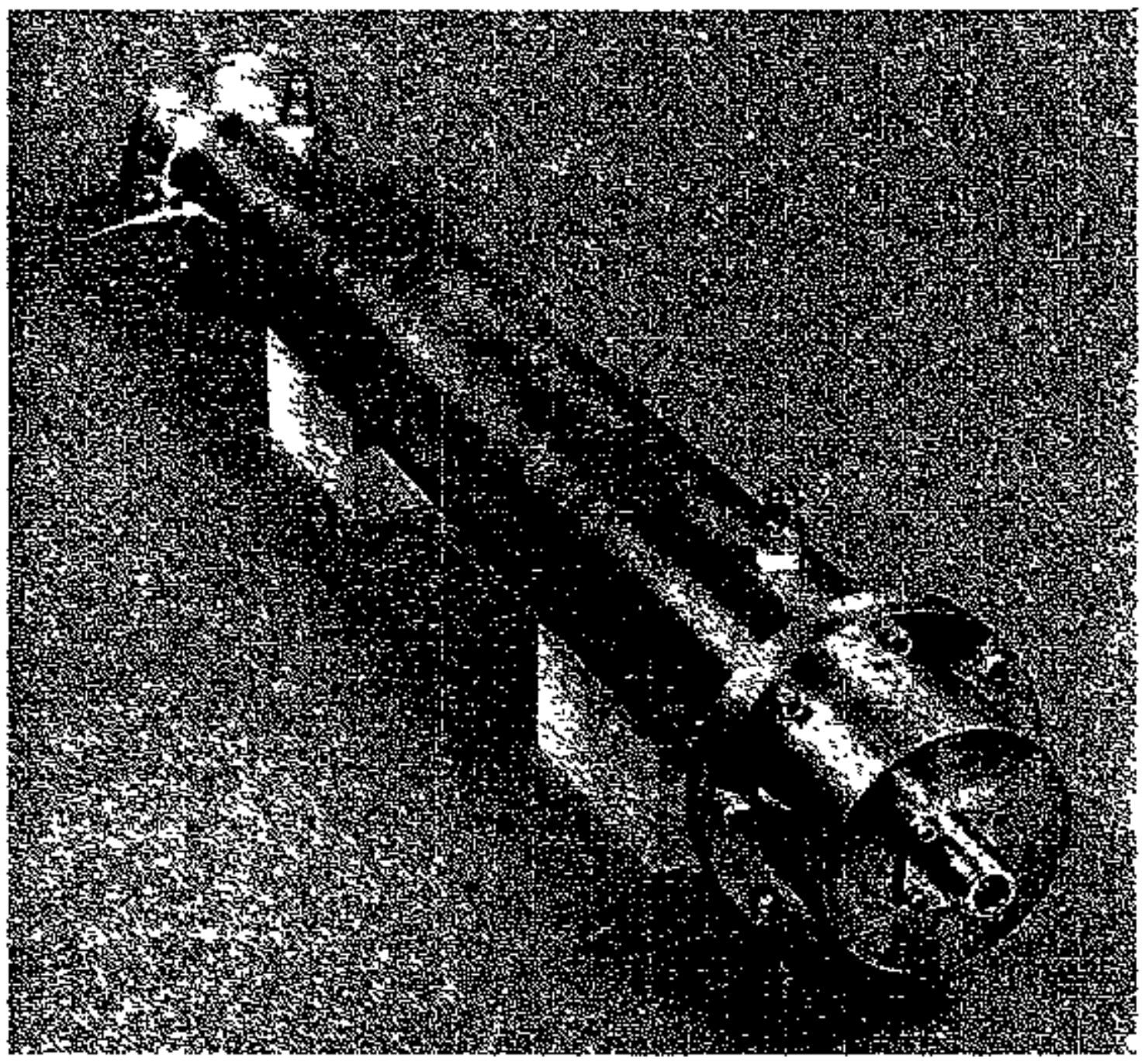

Fig. 9-4. Photograph of the liquid travelling wave transmission cell. Optical fiber leads exit the cell through sealed holes in the cell outer conductor. Fill and vent holes for the liquid are located on top of the cell. 


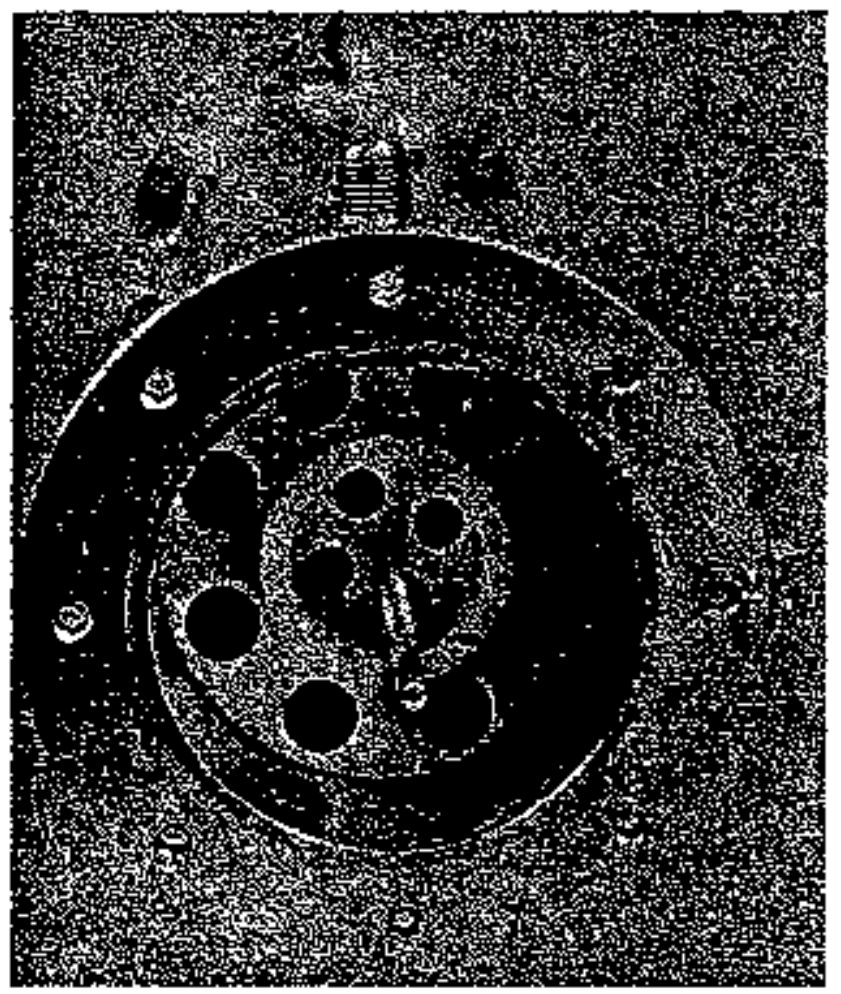

(a)

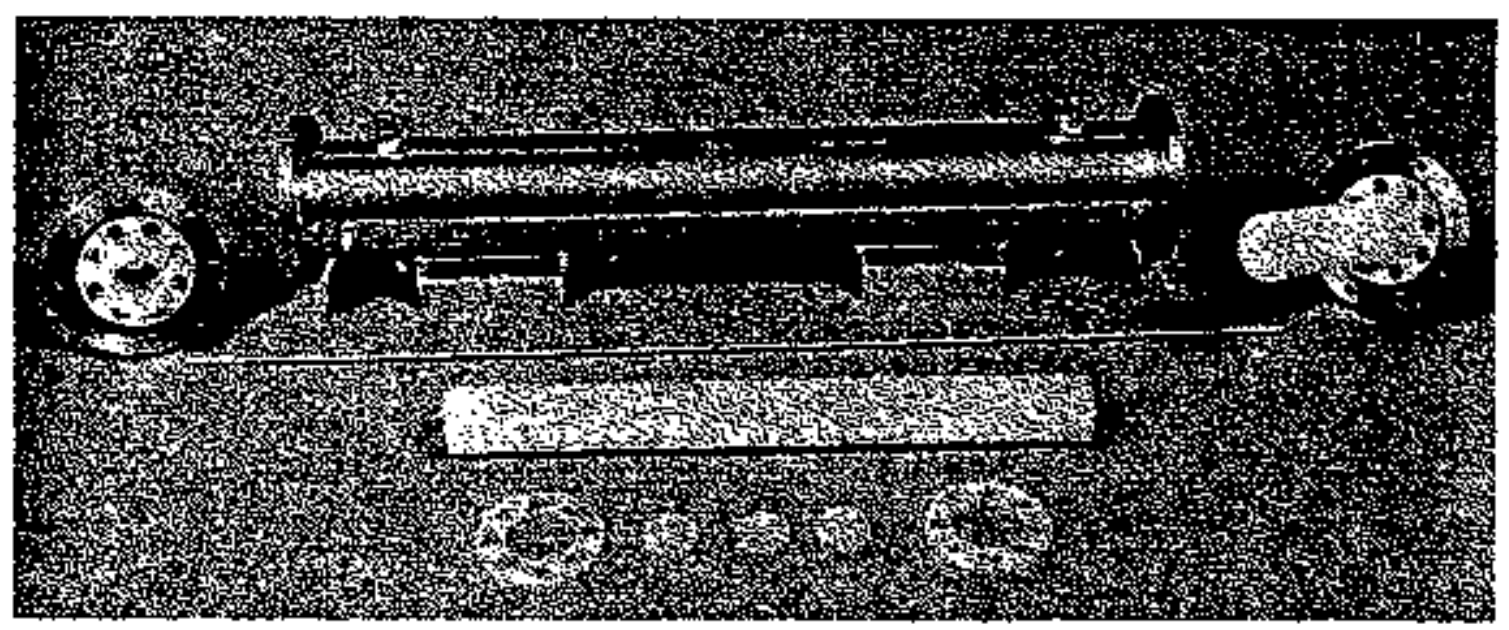

(b)

Fig. 9-5. Photographs of the liquid travelling wave transmission cell. (a) End view showing the inside of the cell, and (b) the fully disassembled cell. 
locations inside the cell. Also, the geometric transition from the RF connectors to the inside of the cell was difficult to design considering the large permittivity steps, the varying size of the small diameter center conductor, and the presence of the support structures. As a result, the SWR of many of the cell configurations was not good.

The measured SWRs for a propanol-filled cell and a water-filled cell are shown in Figs. 9-6 and 9-7. For the propanol cell, the SWR never exceeds 1.31 below $500 \mathrm{MHz}$. These low-level reflections are attributed to the good impedance matching achieved in

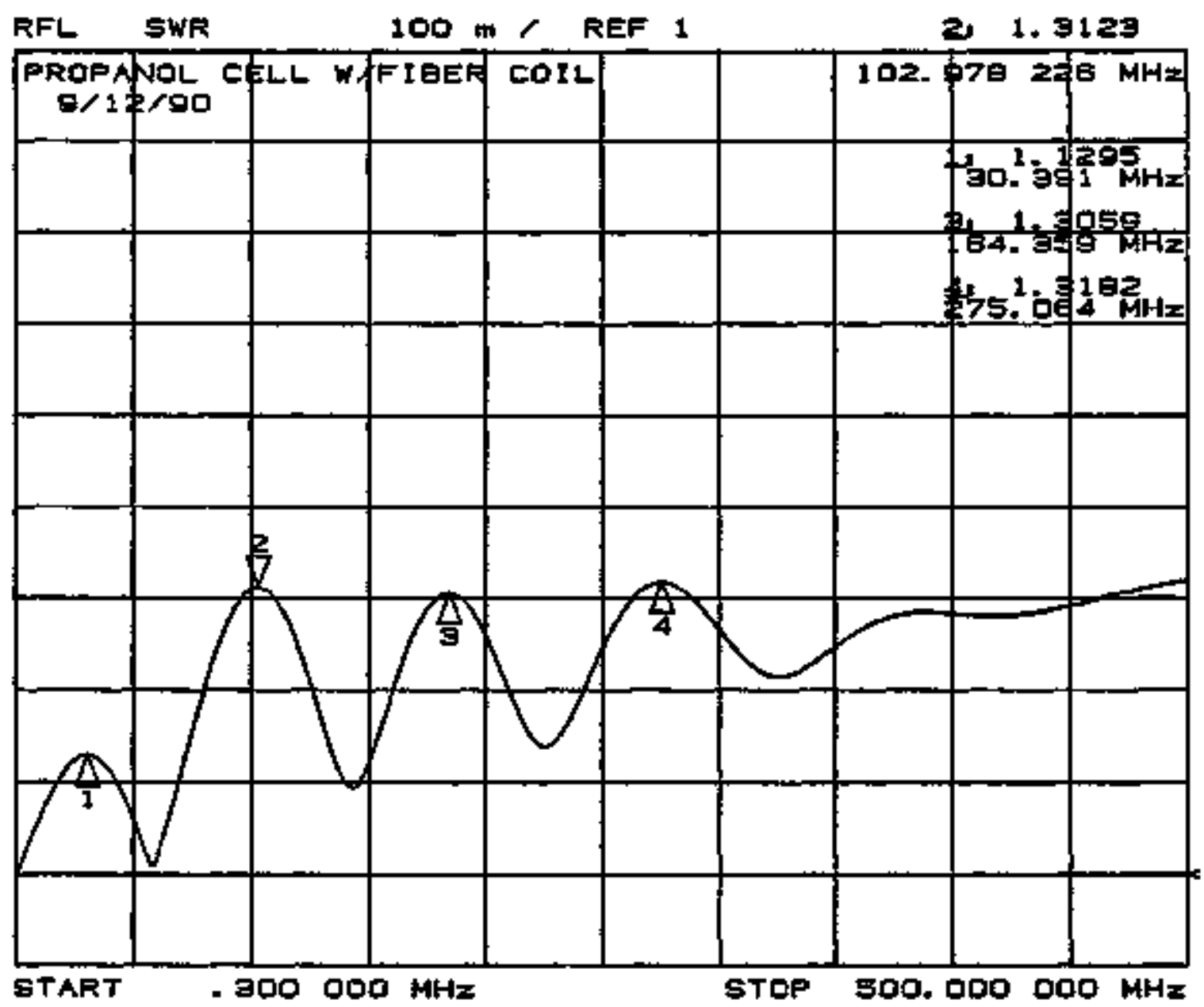

Fig. 9-6. The SWR for the propanol-filted transmission test cell for frequencies op to $500 \mathrm{MHz}$. 
the cell when using a relatively low $\varepsilon_{\mathrm{f}}$ liquid material. The impedance magnitudes corresponding to data in Fig. 9-6 vary between $38 \Omega$ and $52 \Omega$, which represents a maximum deviation of only $\mathrm{L} .2 \mathrm{~dB}$ from the nominatly $50 \Omega$ design.

In contrast, the SWR for the water cell, Fig. 9-7, is as high as 15 for frequencies below $1 \mathrm{GHz}$. This cell is burdened with large reflections due to the high $\varepsilon_{\mathrm{r}}$ of the water. Corresponding impedance magnitudes vary from $-3.5 \Omega$ to $109 \Omega$, a 11 dB deviation from $50 \Omega$. Some configurations of the water cells had better electromagnetic

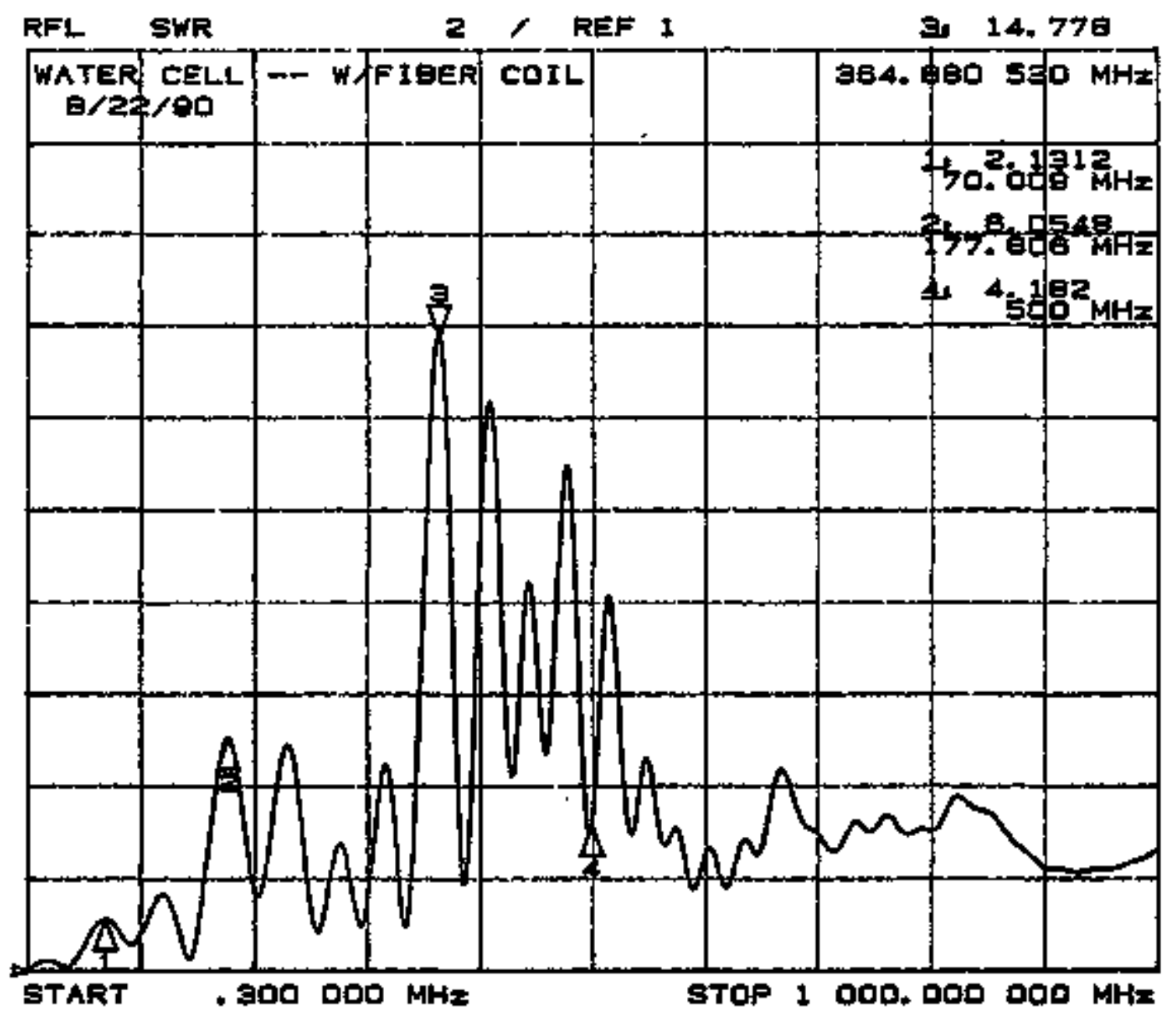

Fig. 9-7. The SWR for the water-filled transmission test cell for freguencies up to 1 $\mathrm{GHz}$. 
characteristics than those shown in Fig 9-7, but all had SWRs greater than 5.0. Even with the large mismatches, however, the water-filled cell proved useful in travelling wave corrent sersor testing. It is postulated that energy, once coupled into the cell, propagates with a wave impedance that closely approximates that of a TEM wave and with a well-established phase velocily that can be estimated by Eq. (3-6). Forther details on the constraction and characterization of the liquid travelling wave cells are found in Ref. 34. 


\section{CHAPTER 10}

\section{EXTENDED MODELS FOR LIQUID CELL TRA VELLING WA VE SENSORS}

The theory for the travelling wave current sensor presented in Chapter 8 assumes all parameters of the fiber coil, the transmission cell, and the dielectric medium are ideal (i.e., they are independent of frequency). In reality, however, several factors contribute to perturbations of the basic travelling wave Faraday response, Eqs. (8-5), all which reduce the preficted bandwidth enhancement given by Eq. (8-10). Infinite bandwidths are certainly not possible, even under the best matching of parameters, but the perturbations do not preclude achieving large bandwidths in many practical devices.

Five mechanisms have been identified that affect the Faraday response in the experimental travelling wave structures and limit the system bandwidth. First is the presence of the fiber coil leads. In the cell confignrations discussed in the previous chapter, the fiber is allowed to spool off the end of the support mandrel and exit the cell through the outer conductor wall. In most cases the fiber leads do not conform to the prescribed helical pitch of the coil proper and merely increase in radial distance along a path that is perpendicular to the conductor. Thus, the leads do not "travel" like the remainder of the fiber and contribute to a minor bandwidth reduction. The deviation in response created by the fiber coil leads is discussed in detail in Appendix B for the planar coil configuration. Since the fiber leads used with the travelling wave coils do not differ significantly from those of the planar coil, the theory presented in Appendix B is adequate to describe all Faraday responses. Also, the Faraday response in the leads is independent of the value of $\varepsilon_{t}$ because of the choset configuration, and thos, both the airline and the liquid cell travelling wave sensor systems experience some bandwidth reductions.

The second limiting mechanism is the modal structure of the coaxial transmission line. All response models, especially those for the travelling wave sensors, are based on 
TEM wave propagation, and more importantly, on the magnetic field component being perpendicular to the direction of wave propagation. Higher order modes begin propagating in the cells at frequencies defined by Eq. (A-13) in Appendix A. This prediction assumes that an ideal dielectric is present in the coaxial waveguide. For the cell configurations described in Chapter 9, the onset of the higher order modes will occur at $2.32 \mathrm{GHz}$ for $\varepsilon_{\mathrm{r}}=1$ (air dielectric), $517 \mathrm{MHz}$ for $\varepsilon_{\mathrm{r}}=20.1$ (the static value for propanol at $25{ }^{\circ} \mathrm{C}$ ), $406 \mathrm{MHz}$ for $\varepsilon_{\mathrm{r}}=32.6$ (methanol), and $262 \mathrm{MHz}$ for $\varepsilon_{\mathrm{r}}=78.5$ (water). The first higher order mode in the ideal coaxial line is $\mathrm{TE}_{11}$, which means the magnetic field will have a component in the direction of propagation - along the direction of the conducted current. This could enhance the Faraday response in the travelling wave coil at the higher frequencies since the light and field vectors might align more readily. But an exact mathematical description of this interaction is difficult since the contribution of both the TEM and the $\mathrm{TE}_{11}$ mode must be considered and each has a separate propagation constant that varies differently with the frequency. Consegpently, the wave phase velocity varies with frequency, and this quantity is the determining factor in the degree of phase matching that occurs in the travelling wave sensor. As more higher order modes are added in the waveguide volume, the situation becomes even more complicated. Thus, operation of the travelling wave current sensors is targeted for a frequency range in which a single dominant waveguide mode exists, limiting the useful bandwidth of the systern.

A third perturbation arises from the presence of the teflon fiber sapport mandrel used in the liquid cell. The relative permittivity of the teflon is $\sim 2.0$ which is much smaller than that of any of the liquids. This modifies the wave propagation in the coaxial line and produces an effective relative permittivity (at low frequencies) for the composite dielectric structure. The effective relative permittivity is smaller than the $\varepsilon_{\gamma}$ of the immersing liquid. The fourth mechanism is liquid dispersion and absorption. Polar molecules that necessarily give rise to the large relative pernittivities must reorient in the 
oscillating fields. At higher freguencies the liquid molecule cannot keep up with the changing field polarization, resulting in absorption of the RF power and a subsequent loss in current detection sensitivity. Finally, a fifth mechanism is described by a combination of those effects just discussed for the second, third and fourth mechanisms above. This response perturbation is due to the waveguide modal stracture in multilayer, dispersive dielectric media.

A detailed description of these last three mechanisms is warranted given their significant affect on the Faraday response of the travejling wave fiber current sensor. Each is treated separately in the following sections. A complete computational model for predicting responses of the liquid travelling wave sensors is offered at the end of this chapter.

\section{Effective. Relative Permittivities}

The relative permittivity of the dielectric medium in the coaxial transmission line establishes the phase velocity of the propagating magnetic wave according to Eq. (3-6). Conseguently, the response, Eqs. (8-5), and the bandwidth, Eq. (8-7), of the travelling wave fiber current sensor are dependent on the value of $\varepsilon_{r}$. In all of the previous theoretical developments, it is assumed that $\varepsilon_{t}$ is single-valued and equal to that of the dielectric medium, here, the liquid. However, in the liguid cell configurations, it is necessary to support the helical fiber coil at a given diameter and position relative to the coax center conductor. When the tefion mandrel tube is submersed in the liquid, the field spatial distribution is perturted from that of the liquid alone, and the EM properties of the cell are changed. The propagating wave in the transmission line then has a phase velocity determined by an apparent or effective relative permittivity which is intermediate to the liquid $\left(\varepsilon_{\mathrm{r}}>20\right)$ and the mandrel $\left(\varepsilon_{2} \sim 2\right)$. For the purpose of computing cell geometric parameters and estimating sensor responses, this effective relative permittivity, $\varepsilon_{\text {eff }}$ is treafed as a single-valued quantity. 
With the fiber mandrel present, the lowest-order mode in the coaxial cell is no ionger TEM, but $\mathrm{TM}_{\mathrm{01}}$. This follows from the waveguide modal solutions for the multilayer dielectric which is treated later in this chapter. In the static or low frequency case, however, the $T M_{01}$ mode can be considered "guasi-TEM," and the electric field has a $1 / p$ dependency in the cell volume between the conductors. The derivation of an effective relative permittivity for a caaxial cell with two dielectric medja, one submersed in the other, is performed in detail in Appendix C. The solution is given as

$$
\varepsilon_{\text {eft }}=\varepsilon_{1}\left[1+\left(\frac{\varepsilon_{1}}{\varepsilon_{2}}-1\right) \frac{\ln \left(T_{2} / 1\right)}{\ln (b / a)}\right]^{-1} .
$$

where $E_{1}$ is the relative permittivity of the liquid, $\varepsilon_{2}$ is the relative permittivity of the mandrel material (the teflon), $r_{1}$ and $r_{2}$ are the inner and outer radii of the mandrel tube, and ' $a$ ' and ' $b$ ' are the center and outer conductor radii of the coaxial line.

Fixed values were designed for three of the radii in the travelling wave liguid cell: $r_{i}=0.475$ in. $(1.20 \mathrm{~cm}), r_{2}=0.625 \mathrm{in}$. $(1.59 \mathrm{~cm})$, and $b=1.13$ in. $(2.87 \mathrm{~cm})$. The value of ' $a$ ' varies with each liquid. When the fixed radii values and the relative permittivity of the teflon mandrel are substituted into Eq. (10-1), the effective relative permittivity becomes

$$
\mathrm{E}_{\text {eff }}=\frac{\varepsilon_{1}}{1+\left(0.5 \varepsilon_{1}-1\right) \frac{0.27}{\ln (1.13 / 2)}},
$$

where 'a' has the units of inches. Figure 10-1 is a plot of Eq. (10-2) for each of the three test liquids used in the cell and with the cell center conductor diameter, $2 a$, as the abscissa. A wide range of values for $2 \mathrm{a}$ are plotted. For the small conductor sizes, the 
effective relative permittivity is greatest, but it is still significantly less than that of the liquid alone, For large conductor sizes, the three carves begin to converge, attaining values of $\varepsilon_{\text {ef }}$ between 5 and 6 at the maximum center condactor diameter, $a=r_{1}$.

The large effect of the teflon fiber mandrel tube on the relative permittivity of the liquid cell can be interpreted by examining the boundary conditions at the dielectric interfaces. From Eq. $(C-5), E_{2}=\left(\varepsilon_{1} / \varepsilon_{2}\right) E_{t}$ at both $r_{1}$ and $r_{2}$. This requires the electric field in the volume occupied by the mandrel to be at least 10 times larger (since $\varepsilon_{1}>20$ and $\varepsilon_{2}=2$ ) than the field in the equivalent volume occupied by the liquid alone. If the relative permittivity of the fiber support material more closely matched that of the

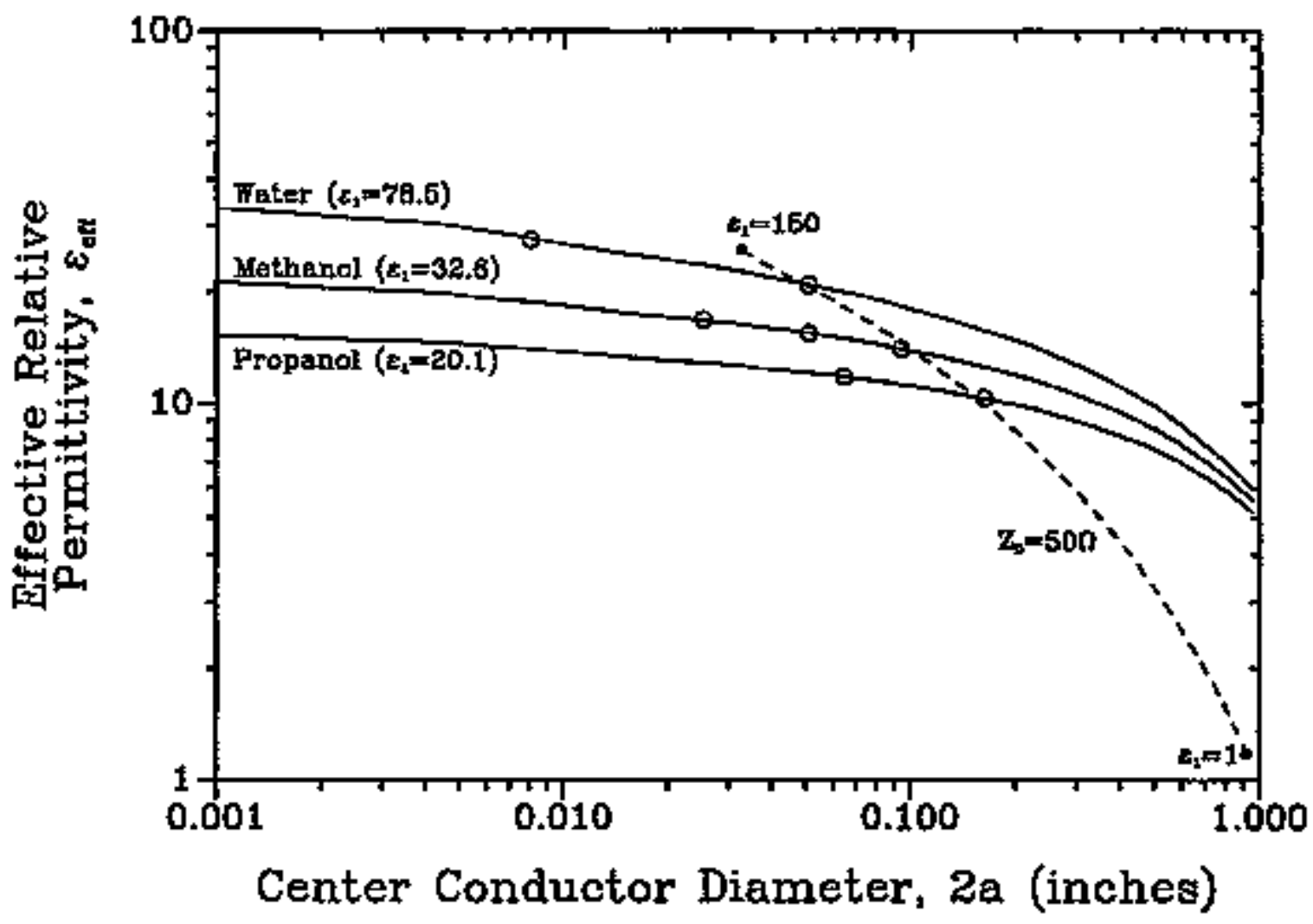

Fig. 10-1. The static, or low frequency, effective relative permittivity versus center conductor diameter for the three liquid traveling wave test cells. The dashed curve represents the appropriate $\varepsilon_{\text {eff }}$ for a coaxial cell with a fixed $50 \Omega$ characteristic impedance. Open circles are parameters for actual experimental cell configurations. 
liquids, the perturbation would not be so great Fortunately, a second boundary condition, $\mathrm{H}_{41}=\mathrm{H}_{42}$, requires the magnetic field component to be uniformly continuous along the cell radius between the two conductors, extending through the teflon. Since the desired current measorements depend only on the interaction of this magnetic field component with the light in the fiber, no deviations will occur in the low frequency Faraday response magnitude even with the fiber at the outer surface of the support mandrel. Thus, the properties of the travelling wave sensor must be adjusted to account only for the faster wave phase velocity created by changes in the relative permittivity.

The low freguency characteristic impedance, $\mathrm{Z}_{0}$, of the liquid travelling wave cell can be computed by substituting $\varepsilon_{\text {eff }}$ from Eq. (10-1) for $\varepsilon_{q}$ in Eq. (A- 11):

$$
Z_{0}=60 \sqrt{\frac{1+\left(\frac{\varepsilon_{1}}{\varepsilon_{2}}-1\right) \frac{\ln \left(T_{2} / t_{1}\right)}{\ln (b / a)}}{\varepsilon_{1}}} \ln \left(\frac{b}{a}\right) .
$$

The relationship between impedance and relative conductor radii is now much more complicated. If all the cell geometric parameters and the material relative permittivities are known, the characteristic impedance can be computed. However, $\mathrm{it}$ is often desirable to identify an impedance, then design the coaxial cell for given dielectric materials. Rearranging Eq. (10-3) gives an expression for the ratio of the conductor radii as a function of the remaining parameters,

$$
\ln \left(\frac{b}{a}\right)=\frac{1}{2}\left(1-\frac{\varepsilon_{1}}{\varepsilon_{2}}\right) \ln \left(\frac{r_{2}}{r_{1}}\right)+\sqrt{\frac{1}{4}\left(\frac{\varepsilon_{1}}{\varepsilon_{2}}-1\right)^{2}\left[\ln \left(\frac{r_{2}}{r_{1}}\right)\right]^{2}+\varepsilon_{1}\left(\frac{Z_{0}}{60}\right)^{2}} .
$$

For a cell matched to $50 \Omega$ and with the teflon mandrel and cell outer conductor 
geometries as given previously, Eq. (10-4) reduces to

$$
a=1.13 \times \exp \left[0.14\left(0.5 \varepsilon_{1}-1\right)-\sqrt{0.02\left(0.5 \varepsilon_{1}-1\right)^{2}+0.7 \varepsilon_{1}}\right] .
$$

This expression can be used to uniquely determine the size of the center conductor required for a $50 \Omega$ test cell for a given liquid. For the three test liquids selected for use in the travelling wave sensors, the center condnctor diameters are $0.15 \mathrm{in} .(0.39 \mathrm{~cm})$ for propanol, 0.1 in. $(0.26 \mathrm{~cm})$ for methanol, and 0.05 in. $(0.13 \mathrm{~cm})$ for water. If these vajues are appropriately substituted into Eq. (10-2), the effective relative permittivities for the $50 \Omega$ cells are 10.5 for the propanol cell, 13.9 for the methanol cell, and 21.1 for the water cell.

In Fig. 10-1, a dashed curve is plotted that represents the values of $\varepsilon_{\text {eff }}$ as a function of 2a for all possible liquids used in our $50 \Omega$ cell. This curve is generated by first selecting a liguid relative permittivity, $\varepsilon_{1}$, and asing Eq. (10-5) to find 'a'. The values for $\varepsilon_{1}$ and ' $a$ ' are then substituted into $\mathrm{Eg}$. (10-2) to find a unigue $\varepsilon_{\text {effr- Liquid }}$ relative permittivities cover the continan from $\varepsilon_{1}=1$ to 150 . These extreme values for $\varepsilon_{1}$ are shown as the endpoints for the dashed curve in Fig. 10-1. The intersections of the dashed curve with the three solid curves identify the values of the effective relative permittivity for the $50 \Omega$ cells for the selected test liquids. These three values were stated in the preceding paragraph. Also plotted in Fig. 10-1 (as the open circies) are the parameters of the actual travelling wave sensor experimental configurations. As can be seen, for each test liquid a configuration near $50 \Omega$ characteristic impedance was used. Other cell configarations with different impedances were also inaplemented.

Equation (10-1) can also be used to compute the effective relative permittivity of the dielectric in the airline travelling wave test cell. This cell used a polyurethane foam $\left(E_{\mathrm{r}}<1.2\right)$ mandrel to support the helical optical fiber. As might be expected, the foam 
represents only a minimal perturbation on the cell dielectric properties: $\varepsilon_{c f f}=0.98$ if $\varepsilon_{2}=$ 1.1, and $\varepsilon_{\text {eft }}=0.96$ if $\varepsilon_{2}$ is as large as 1.2 . Since the transmission line characteristic impedance follows as $\left(\varepsilon_{\mathrm{eft}}\right)^{-1 / 2}$, insignificant changes in the wave properties occur dae to the presence of the foam fiber mandrel.

\section{Liquid Dispersion}

One of the greatest concerns with liguid as a dielectric medium is the dispersion that occurs at high frequencies. The EM wave propagating in the medium causes oscillations of the liguid molecule. At high frequencies, these molecules cannot reorient fast enough to move synchronously with the field. A phase delay occurs between the wave and the molecule resulting in apparent deviations of the real component of the relative permittivily and significant increases in the imaginary component. The latter effect gives rise to dielectric absorption. The complex relative permittivity is expressed by Eq. (A-16). A Debye relaxation model is used to describe the real and imaginary components in terms of the molecular properties [71]:

$$
\varepsilon_{\mathrm{r}}=\left(1+\frac{4 \pi \alpha_{s} \mathrm{~N}_{\mathrm{d}}}{1+\omega^{2} \mathrm{~T}_{\mathrm{R}}^{2}}\right)+\mathrm{j}\left(\frac{4 \pi \alpha_{\mathrm{s}} \mathrm{N}_{\mathrm{d}}}{1+\omega^{2} \mathrm{~T}_{\mathrm{R}}^{2}} \omega \mathrm{T}_{\mathrm{R}}\right),
$$

where $\alpha_{s}$ is the static polarizability of the molecule, $N_{\alpha}$ is the molecular density, and $T_{R}$ is the molecular reorientation time. $T_{R}$ is closely related to the material viscosity and the size of the molecule [71]. Smalt liquid molecules with low viscosity, such as water, will produce large absorption only at the highest frequencies; however, dielectrics with large molecules will exhibit significant dispersion.

Figure 10-2 shows the frequency characteristics of the real ( $\left.\varepsilon^{\prime}\right)$ and inaginary $(\varepsilon ")$ parts of Eq. (10-6) for a typical dielectric medium. When the frequency is eqqal to $1 / T_{R}$, the imaginary component is maximum (indicating peak absorption) and the real 


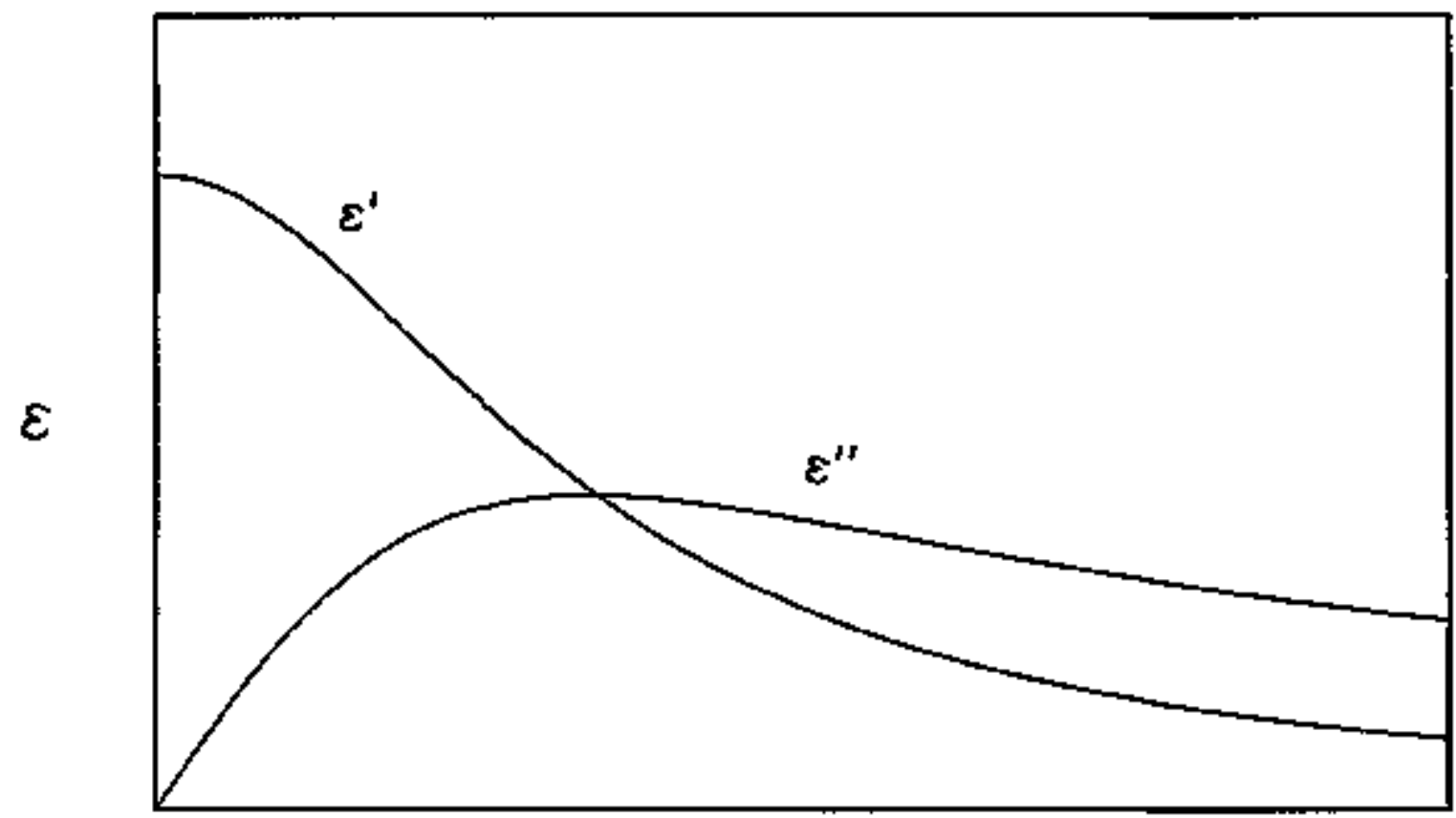

\section{$\omega$}

Fig. 10-2. The real and imaginary components of the relative permittivity for a dispersive medium.

component is reduced to approxiratety one-half the difference between the low freguency and the high frequency relative pernitivity. As an example, the peak absorption for water occurs near $16 \mathrm{GHz}$ at $25{ }^{\circ} \mathrm{C}$ which equates to a molecular reorientation time of $T_{R} \sim 10^{-11}$ seconds [72]. The values of the reorientation time for the other two liquids used in the experimental travelling wave sensor cells are approximately $3.6 \times 10^{-10} \mathrm{sec}$ for propanol and $7 \times 10^{-11} \mathrm{sec}$ for methanol. These two liguids have much larger molecules than water, conseqwently, the longer reorientation times. In many materials, several higher frequency absorption bands will also occur. Each of these will have relative permittivity characteristics as shown in Fig. 10-2, only with progressively lower starting values for $\varepsilon^{\prime}$. For the purpose of describing dispersion in the liquid dielectrics used in this study, however, only the first absorption is of interest.

The static or Iow frequency value for the relative permittivity, $\varepsilon_{s}$, is found from 
Eq. $(10-6)$ when $\omega=0$ :

$$
E_{s}=1+4 \pi \alpha_{s} N_{d}
$$

Static values for materials are usually those published in the handbook tables. If a constant parameter, $\kappa$, is defined as $\kappa$ is $4 \pi \alpha_{3} N_{d}$ then $\kappa=\varepsilon_{s}-1$. The two material dielectric parameters, $K$ and $T_{R}$, are all that is needed to fully describe the frequency dependence of the relative permittivity given by Eq. (10-6).

The amplitude of the propagating EM wave in the coaxial transmission line is given by

$$
A(z)=A_{0} e^{-\beta z}=A_{0} e^{-j \frac{\omega}{c} \sqrt{\varepsilon_{r}} z}
$$

where $A_{0}$ is the initial wave amplitude, $\beta$ is the waveguide propagation constant, and $z$ is the direction of propagation. The attenuation or absorption coefficient is determined from the real component of the exponential factor, or

$$
\alpha_{D}=\frac{\omega}{c} \operatorname{Im}\left(\sqrt{\varepsilon^{\prime}+j \varepsilon^{\prime \prime}}\right) .
$$

If attenuation is small, the dielectric has a loss that is described by Eo. (A-18). For the liquids at higher frequencies, larger attenuation occurs and Eq. (10-9) must be used directly to deternine losses. Figure 10-3a shows the transmission magnitude measured for the travelling wave sensor cell filled with propanol. The dashed curve is a prediction of the frequency-dependent absorption using the wavegoide model discussed in the following section of this chapter. Absorption is quite large in this configmration, approximately $30 \mathrm{~dB}$ at $500 \mathrm{MHz}$. The Foraday response sensitivity for the travelling 
(a)

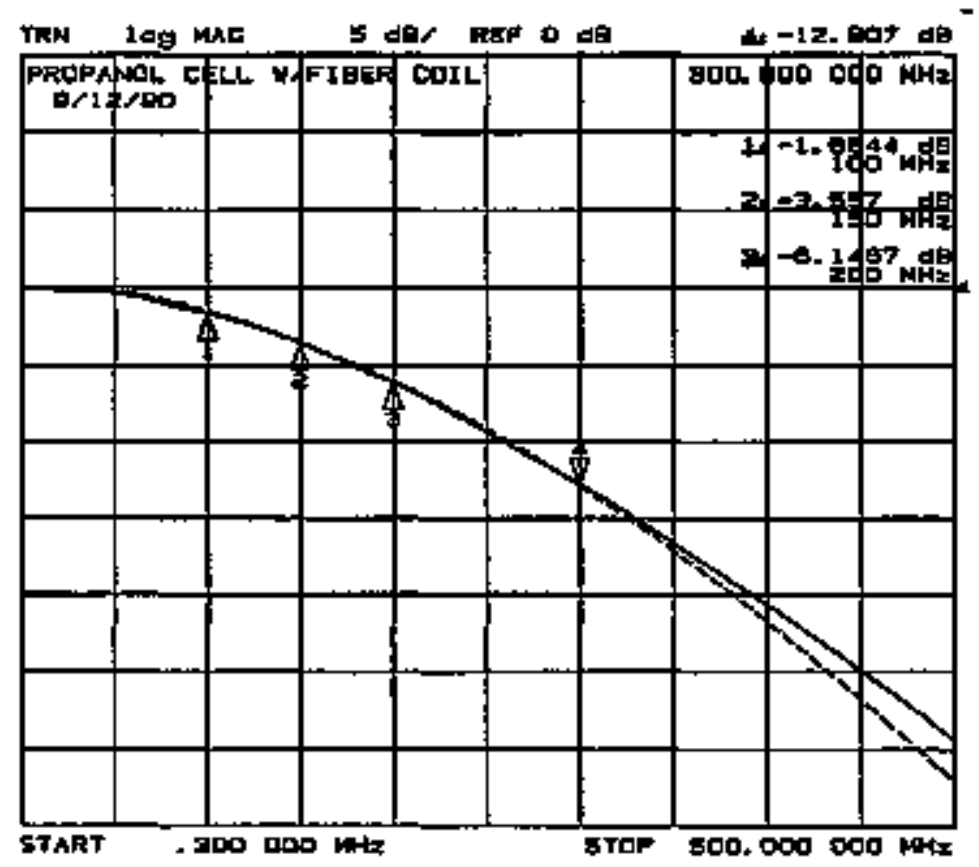

(b)

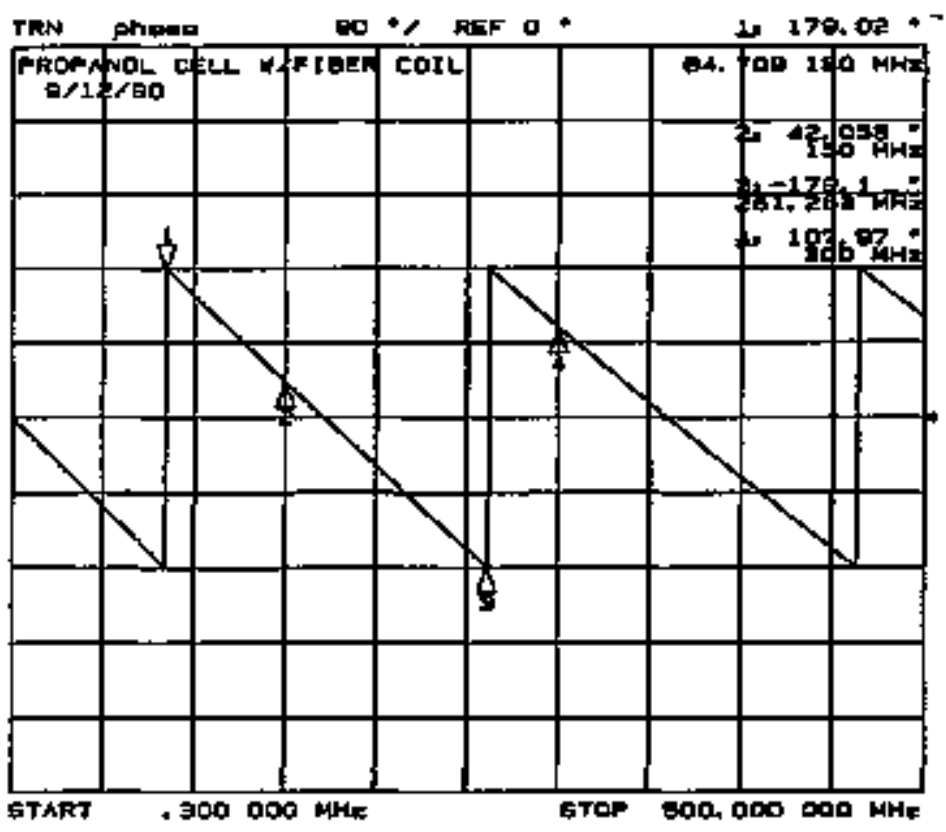

Fig. 10-3. The transmission magnitude (a) and phase angle (b) for the propanol-filled liquid test cell used as a travelling wave sensor. The dashed curve in $(a)$ is a computation of the cell attenuation produced by absorption in the propanol with an estimated molecular reorientation time of $3.6 \times 10^{-10} \mathrm{sec}$. In (b) the liquid dispersion creates a noticeable phase lag. 
wave sensor is significantly diminished under these large attenuation conditions. In fact. it is determined that only $\sim 20 \mathrm{~dB}$ of loss can be accornmodated before measurement signal-to-noise ratios become too small. This limitation then establishes a higher frequency bound on each travelling wave sensor configuration. For the propanol-filled cell, this upper frequency limit is approximately $380 \mathrm{MHz}$. For the methanol cell, the linit is $\sim 650 \mathrm{MHz}$, and for the water cell, $\sim 825 \mathrm{MHz}$. Because of other limitations in the Faraday current sensor response, none of the cells could be used up to this absorption frequency bound.

Absorption of the RF power by the liquid dielectrics limits the maximum energy that can be injected into the transmission cells. The heat generated by the absorbed power elevates the liquid temperature, thus lowering the relative permittivity (see Tables 9-1 and 9-2). No attempts were made during experimentation to control the liquid temperature, but a monitor was used and the temperature was continuously recorded. The controller for the fiber optic temperature sensor, a Luxtron Model 1000A, can be seen in the lower left of the photo in Fig. 9-1. Liguid temperatures were not allowed to deviale greater than $5^{\circ} \mathrm{C}$ during all tests. This ensured a reasonably stable relative permittivity and cell impedance, and negated the need for any corrections to the measured sensor responses.

In addition to absorption, dispersion in the liquid relative permittivity also perturbs the waveguide propagation parameter. At the higher frequencies, changes in both $\varepsilon^{\prime}$ and $\varepsilon^{\prime \prime}$ produce deviations in the imaginary component of the exponential factor in Eq. (10-8). This factor is critical in establishing the wave phase velocity in the travelling wave sensor configuration. In liquids that exhibit large absorption, large increases in phase velocity also occur. In Fig. 10-3b, the transmission phase angle for the propanolfilled cell is plotted. A noticeable phase lag is observed, which results from the relative permittivity steadily decreasing as the frequency increases. This indicates that the EM wave in the cell is gaining speed with frequency. In a current sensing system this leads 
to a gradually increasing phase mismatch with the light in the optical fiber. Faraday response will obviously decrease at the higher frequencies.

\section{The Liguid Coaxial Wavequide}

An exact determination of the electromagnetic properties for the liquid travelling wave sensor cells requires a detailed antysis of the aciual and complete waveguide. This antalysis must include the relative permittivity perturbations caused by the presence of the fiber mandrel tube, the dispersion in the liquid dielectric, and the waveguide dispersion created by modes at the higher frequencies. The general problem that must be solved is finding the modal structure in the transmission line dielectric volume for a multilayer coaxial dielectric with complex relative permittivities. Maxwell's equations (the wave equations) are solved subject to the appropriate coaxial boundary conditions. From these solutions, a complex relative permittivity is detemined that can be substituted inlo Eg. (8-4) to give an expression for the frequency-dependent Faraday response in the travelling wave cunrent sensor.

A complete, detailed solution to the general wavegaide problem is quite involved; it must assume that all spatially-orthogonal components of the magnetic and electric field exist inside the transmission line dielectric volume. The approach of reducing the computations to TM and TE modes as taken for the ideal coaxial line (see Appendix A) may not be appropriate. Coupled modes must initially be considered. Fortunately, the implementation of the travelling wave sensors only requires knowledge of the lowest order mode propagating in the cells. This can be achieved by simplifying the problem from the outset: assume a limited modal structure and a limited frequency range of operation. Previously, the lowest order mode in the liquid-filled cells was assumed to have quasi-TEM characteristics. Then, a reasonable assumption is that this mode possesses azimuthal symmetry. With no $\phi$ dependencies, the field components in the multilayer dielectric become uncoupled (transverse field components are no longer a 
sugerposition of $E_{z}$ and $H_{y}$ [73], and the modal problem can again be simplified mathernatically into TE and TM groupings. The problem is further simpiified by identifying the approximate cotoff frequency of the first higher order azimuthally symmetric mode, then bounding sensor operation to the assumed single mode region below this freguency. As verified by the experimental data, the above assumptions have proved quite accurate in treatment of the travelling wave fiber sensor response.

The eigenvalue equations for the $\mathrm{TE}_{0, \mathrm{a}}$ and $\mathrm{TM}_{\mathrm{0y}}$ modes in the liquid-filled transmission cells are developed in Appendix D. Modal solutions to these equations are approached, and eventually displayed, from the perspective of an effective relative permittivity, $\varepsilon_{\text {eff }}$ The effective relative pernittivity is related to the waveguide propagation constant through the expression

$$
\beta=\mathrm{j} \frac{\omega}{\mathrm{c}} \sqrt{\varepsilon_{\mathrm{eff}}} .
$$

It is more common to determine the modes in the coaxial transmission line by simply finding $\beta$ as a function of frequency. Such a treatment is used in Appendix A. However, the relative permittivity representation continues from earlier discussions of the travelling wave sensor systems and allows for greater insight into dielectric properties, especially dispersion. The use of an $\varepsilon_{\text {eff }}$ in the mathematical treatment is consistent with other waveguide properties.

Throughout the waveguide modal analysis, it is assumed that computed values for $\varepsilon_{\text {eff }}$ will be representative of a homogenous dielectric medium between the conductors in the coaxial line. Then the effective relative pernittivity can be used to estimate impedances, propagating-wave phase velocities, and ultimately, the travelling wave sensor responses. In the waveguide computations, the liquid relative permittivity is treated as complex, thus the resulting $\varepsilon_{\text {eff }}$ is complex. This allows for treatment of both the wave dispersion and attenuation. 
An iterative computer algorithm [34] was used to compute the solutions to the modal eigenvalue equations, Eq. (D-15) for the $\mathrm{TM}_{0_{\mathrm{s}}}$ modes and $\mathrm{Eq}$. (D-27) for the $\mathrm{TE}_{\mathrm{0}_{\mathrm{g}}}$ modes. The code initially estimates a value for $\varepsilon_{\text {eff }}$ (complex components where appropriate) at a given frequency and then computes the waveguide propagation constant, $\beta$, using Eq. (10-10). The complex value of $k_{1}$ (from Egs. (A-4) and (10-6)) for the liquid dielectric is combined with $\beta$ to determine the parameter $v_{1}$ from Eq. (D-2). Similarly, $\mathfrak{u}_{2}$ is determined from $\boldsymbol{B}$ and $k_{2}$, for a nondispersive relative permittivity for the teflon fiber mandrel. Arguments for the Bessel functions are then generated from the geometric dimensions, and trial solutions to the eigenvalue equations are computed. Iterations of $\varepsilon_{\text {eff }}$ occur until satisfactory solutions are found. Because $u_{1}$ and $u_{2}$ can assume any complex value, it is necessary to atilize Bessel function routines that allow complex arguments. These functions were provided by D. E. Amos as a complete Fortran subroutine package [74].

Modes within the multilayer dielectric transmission line are first identiffed by treating the liquids as lossless or nondispersive. Then the effective relative pemittivities introduced into Eqs. (10-10) and (A-4) are real only. The lowest order mode for all the moltilayer dielectric configerations is $\mathrm{TM}_{01}$. A TEM mode cannot exist. Guided modes in the cell require that $\varepsilon_{2} \leq \varepsilon_{\text {eff }}<\varepsilon_{1}$ for propagation to occur; modes fall below cutoff if $\varepsilon_{e f f}<\varepsilon_{2}$. This implies the phase velocity of the propagating wave is faster than the speed of light in the liquid medium. Such anomalous phase velocities are not nnusual in guided wave structures. The maximum phase velocity in the multilayer dielectric is linited by the speed of light in the teflon.

Figure 10-4 is a plot of $\varepsilon_{\text {eff }}$ versus frequency for several TM and TE modes in a lossless coaxial transmission cell filled with water. At zero frequency, the static value of $\varepsilon_{\text {erl }}$ for the $T M_{01}$ mode agrees exactly with that determined by Eq. (10-2) from the theory developed in Appendix $C$. This validates the assumption that at low frequencies a quasiTEM mode exists in the cell. In Fig. 10-4 the modal values for $\varepsilon_{\text {eff }}$ are bound by $\varepsilon_{1}$ on 


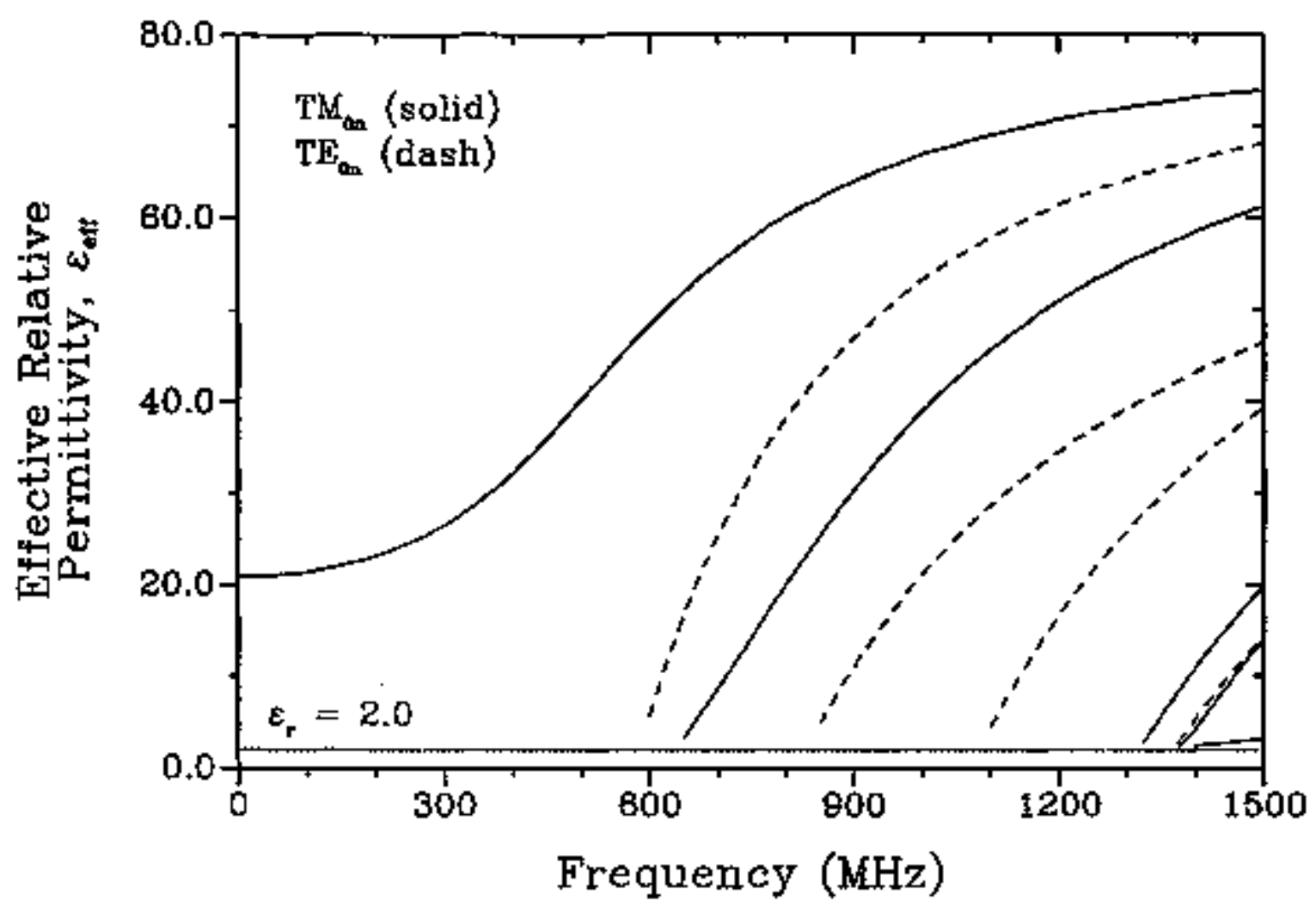

Fig. 10-4. The effective relative permittivity versus frequency for the azimuthally symmetric $\mathrm{TM}_{\mathrm{on}_{\mathrm{n}}}$ and $\mathrm{TE}_{\mathrm{Dp}}$ modes in a multilayer dielectric coaxial waveguide containing water. Liquid dispersion is neglected.

the high side and $\varepsilon_{2}$ on the low side. The transmission cell becomes highly multimoded as the frequency approaches $1.5 \mathrm{GHz}$; several additional modes, that are not azimuthally symmetric, are expected to appear among the $\mathrm{TM}_{0 \mathrm{n}}$ and $\mathrm{TE}_{\mathrm{on}}$ modes at the higher freguencies. The first higher order azimuthally symmetric mode in Fig. 10-4 is the $\mathrm{TE}_{01}$ mode which begins propagating near $600 \mathrm{MHz}$. This catoff establishes an approxinate upper frequency bound for single mode operation in a water-filled travelling wave cell. For travelling wave cells filled with propanol and methanol, the $\mathrm{TE}_{\mathrm{Ol}}$ mode is also the first higher order mode in the multilayer dielectric waveguide assuming nondispersive liquids. The cutoff frequency for this mode is approximately $1220 \mathrm{MHz}$ in the propanol cell and approxinately $940 \mathrm{MHz}$ in the methanol cell. 
Portions of the lower frequency $\varepsilon_{\text {eff }}$ for the $\mathrm{TM}_{01}$ mode in the lossless liquids propanol, methanol and water are plotted as the dotted reference curves in Figs. 10-5 through 10-7. All three curves show a static value for $\varepsilon_{\text {eff }}$ that agxees with the previously determined quasi-TEM calcolations. Also, each curve shows a waveguide dispersion in the effective relative permiltivity that is independent of the liquid dispersion. Below 500 $\mathrm{MHz}$, the waveguide dispersion for the propanol-filled cell is quite small (se Fig. 10-5), but values for the methanol cell and especially the water cell are quite significant (see Figs. 10-6 and 10-7). This waveguide dispersion arises from the large disparity between the relative permittivities of the liquid and the teflon tobe, and subsequently, from the large disparity between the static $\varepsilon_{\text {eff }}$ value and the static liguid $\varepsilon_{\mathrm{r}}$.

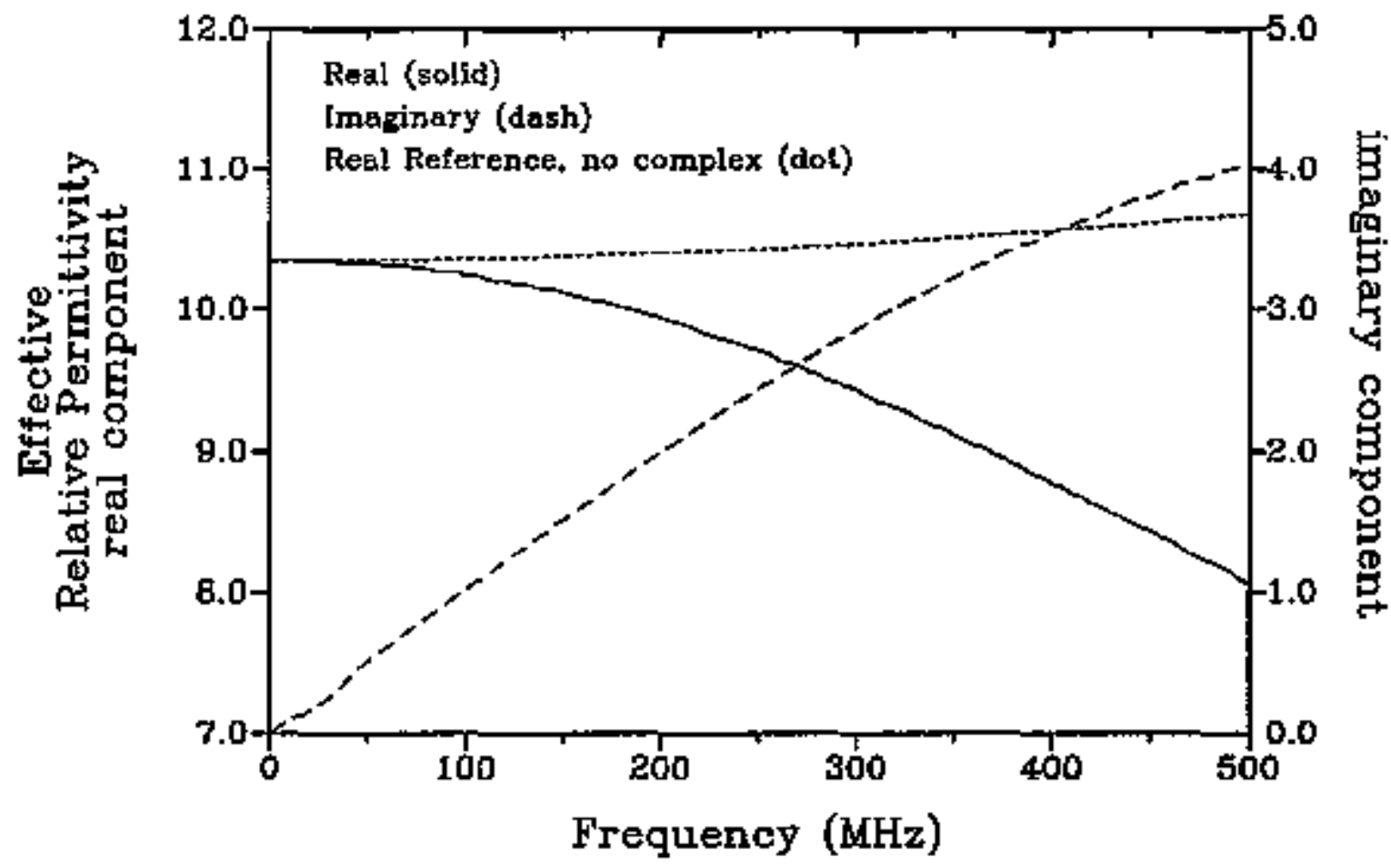

Fig. 10-5. The complex effective relative permittivity versus frequency for the $\mathbf{T M}_{\mathbf{0 1}}$ mode in a propanol-filled travelling wave trassmission cell. The dotted reference curve is produced from the waveguide model neglecting liquid dispersion. 


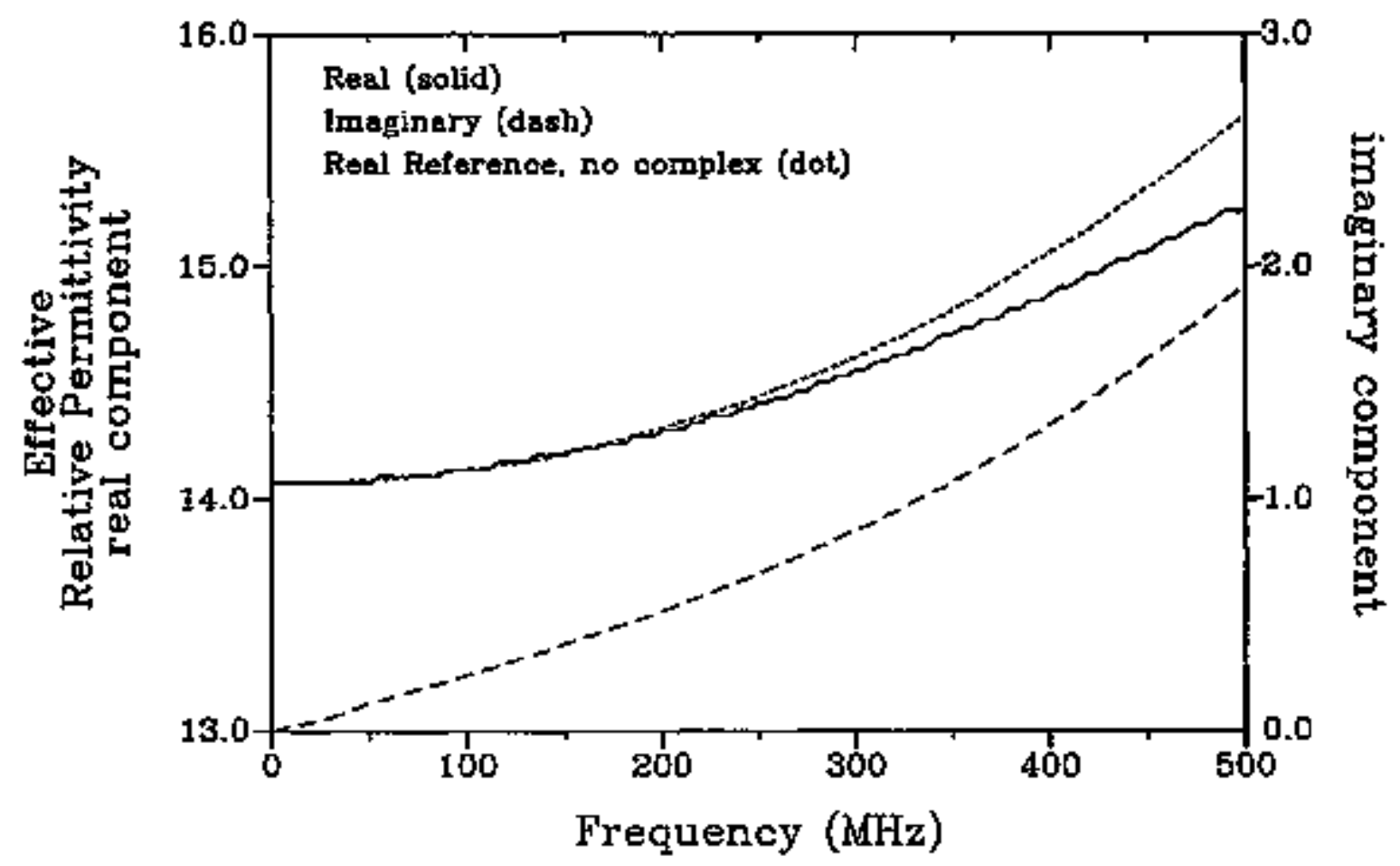

Fig. 10-6. The complex effective relative pennittivity versus frequency for the $\mathrm{TM}_{01}$ mode in a methanol-filled travelling wave transmission cell. The dotted reference curve is produced from the waveguide model neglecting liquid dispersion.

When ligaid dispersion is included in the multilayer waveguide computations, complex solutions for $\varepsilon_{\text {eff }}$ are generated. Only the $\mathrm{TM}_{01}$ mode is investigated for these dispersion calculations, and the frequency range is limited to $500 \mathrm{MHz}$ for the propanol and methanol-filled cells and to $600 \mathrm{MHz}$ for the water-filled cell. Cell absorption is included as part of the computations so that comparisons can be made directly to measured transmission (or loss) values. The molecular reorientation time, $T_{R}$, is treated as a variable input paraneter in the model. The complex relative permittivity of the liguid, Eq. (10-6), is then used to determine $\mathrm{k}$ and $\mathrm{u}$ for use in the wavegoide eigenvalue equations. Differences between computed and measured attenuation values deternine the correctness of the chosen value of $T_{R}$. Figare 10-3a is one such comparison of cell transmission values for the propanol-filled coaxial line. The dashed curve in Fig. 10-3a 


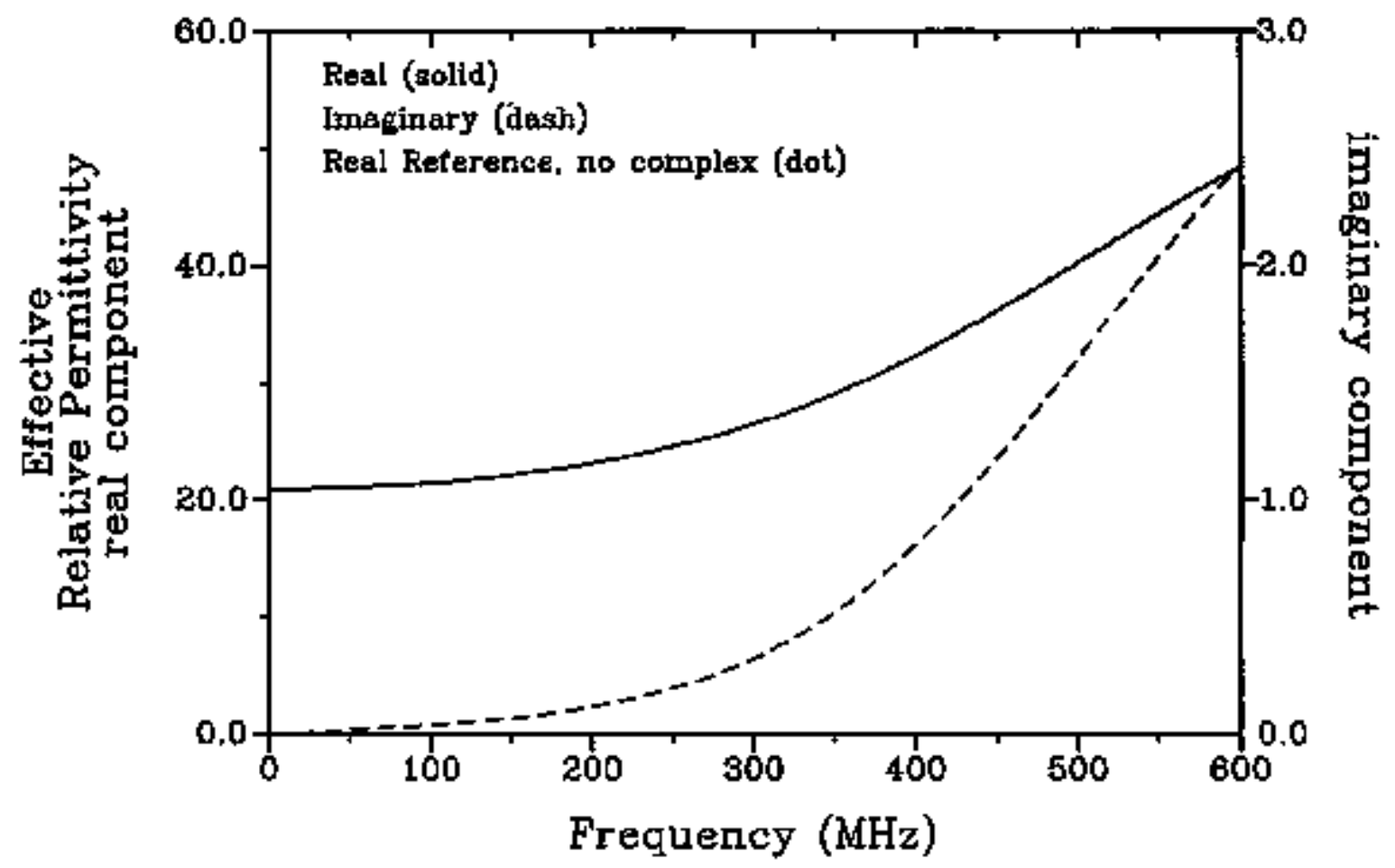

Fjg. 10-7. The complex effective relative permittivity versus frequency for the $\mathbf{T M}_{01}$ mode in a water-filled travelling wave transmission cell. The dotted reference curve, which is almost hidden behind the solid curve, is produced from the waveguide model neglecting liguid dispersion.

is generated by the multilayer waveguide model for $T_{R}=3.6 \times 10^{-10}$ sec. Good agreement with the network analyzer curve is observed, especially below $350 \mathrm{MHz}$. All estimated valnes for $T_{R}$ for the three test liguids were determined using this technigue .

The complex effective relative permittivities versus frequency for the three liquids are plotted in Figs. 10-5 through 10-7. The real and imaginary components of $\varepsilon_{\text {eff }}$ are plotted as separate curves in each figure. In the propanol-filled cell, Fig. 10-5, absorption dominates. The imaginary part of $\varepsilon_{\text {eff }}$ increases rapidly with increasing frequency. The dispersion in the real part of $\varepsilon_{e f f}$ is dramatic; liquid dispersion dorninates the waveguide dispersion which is observed by comparing the solid and dotted curves in Fig. 10-5. In the methanol-filled cell, Fig. 10 6, the absorption is mach smaller below 
$500 \mathrm{MHz}$. Waveguide dispersion is slightly greater than the liquid dispersion, as the real componen. of $\varepsilon_{\text {eff }}$ increases only $8 \%$ over the frequency range plotted. Because of the fast reorientation time for the water molecules, little liquid absorption occurs in this travelling wave cell. In Fig. 10-7, the real component of $\varepsilon_{\text {df }}$ below $600 \mathrm{MHz}$ is almost identical to $\varepsilon_{\text {eff }}$ computed for the lossless liquid (the dotted curve). Waveguide dispersion, however, produces more than a two-fold increase in the value of $\varepsilon_{\text {eff }}$. This leads to dramatic effects in the wave propagation characteristics for the water cell.

The complex effective relative permittivity for each liquid is used to compute the wave inpedance, using Eq. (A-9), for the $\mathrm{TM}_{01}$ mode in the multilayer dielectric transmission line. The $\mathrm{TM}_{01}$ mode is considered quasi-TEM at the low frequencies, which means that over some frequency range the wave inpedance can be considered constant. For a pure TEM mode, the wave impedance does not change with frequency.

Figure 10-8 shows a plot of the wave impedance magnitude for the travelling wave cells filted with the three test liguids. This parameter deviates with frequency in much the same manner as the real components of $\varepsilon_{\text {of }}$ plotted in Figs. 10-5 through 10-7. For methanol in the liquid-filled cell, Fig. 10-8 shows almost a constant wave impedance magnitude up to $500 \mathrm{MHz}$. Waveguide characteristics for this cell configuration are obviously quite good, indicating that travelling wave sensors using methanol as a liquid dielectric are useful up to the liquid absorption frequency limit, approximately $650 \mathrm{MHz}$. For propanol, the wave impedance magnitude shown in Fig. 10-8 deviates somewhat greater (and with opposite stope) than methanol with increasing frequency. If a $10 \%$ deviation from the static valae is allowed in considering the freqnency range over which the wave impedance is "essentially constint", then travelling wave sensors using propanol can operate to $\sim 375 \mathrm{MHz}$. This frequency is about the same as that established by the lignid absorption limit. Waveguide dispersion in the water-filled cell creates large deviations in the wave impedance magnitude as seen in Fig- 10-8. Greater than 10\% increases from the static $\eta$ value are observed for frequencies above $315 \mathrm{MHz}$. This is 


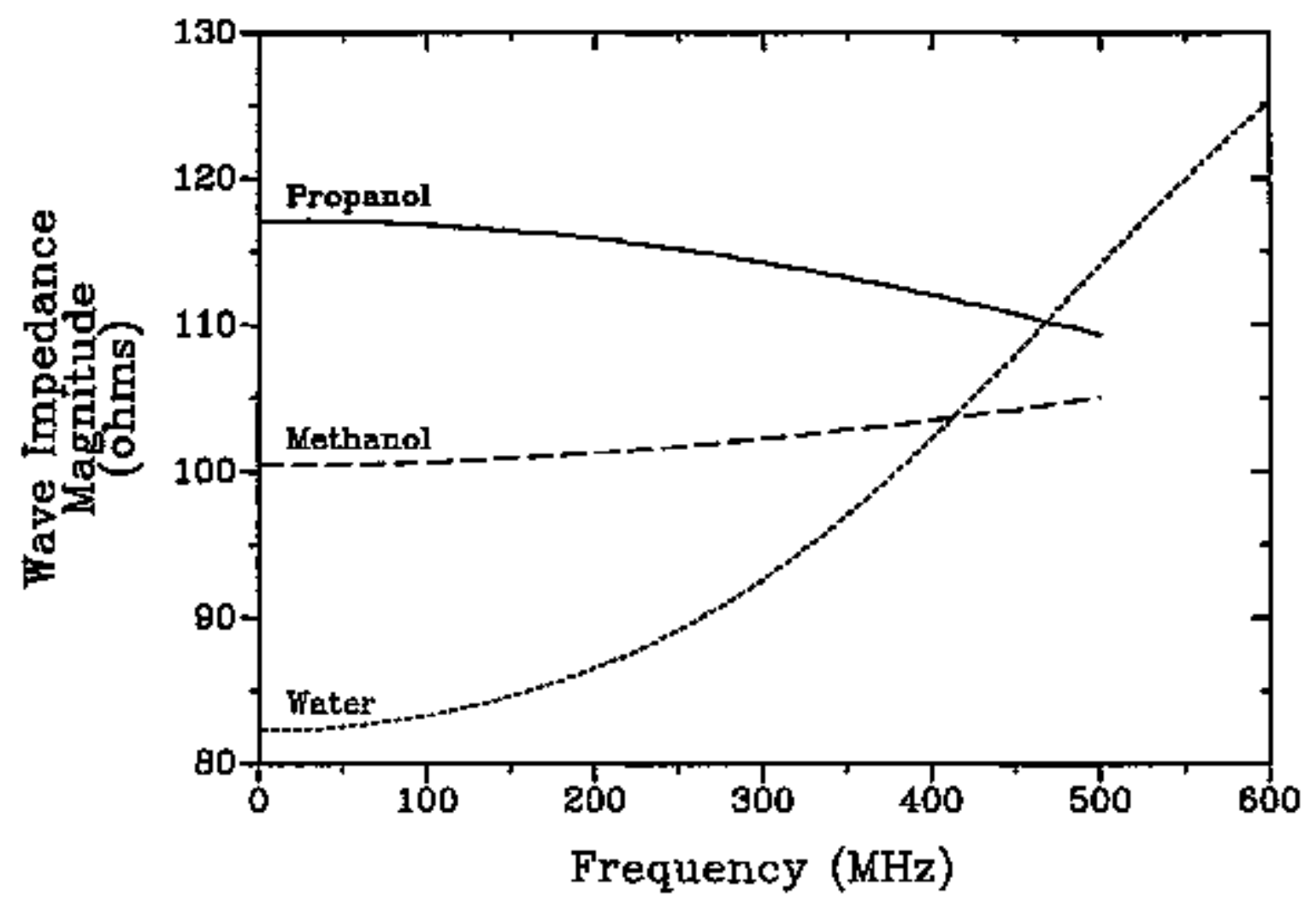

Fig. 10-8. The computed wave impedance magnitude for the $\mathrm{TM}_{\mathrm{Ol}}$ mode in liquid-filled travelling wave cells for each of the three test liquids.

easily the most dominant factor in configuring a single mode cell with water dielectric for use as a travelling wave sensor.

\section{The Complete Sensor Model}

The mechanisms discussed in the previous sections of this chapter significantly alter the electromagaetic properties of the travelling wave sensor cells and, thus, can have a profound effect on the response of the fiber sensor. The relative permittivity of the coaxial dielectric medium cannot be treated as a constant over all the freguencies of interest, and in many cases it deviates largely from its static value and its liquid-only value. Thus, only when dealing with ideal or severely confined configurations can the response function given by $\mathrm{Eq}$. (8-5) be used to describe the frequency dependent 
Faraday rotation. In all other cases, Eq. (8-4) must be used directly to predict responses. A suitable expression for the relative permittivity is identified and sabstituted for $\varepsilon_{\mathrm{r}}$ in Eq. (8-4); integration then follows to determine the response. For the most complete description, the liquid multilayer coaxial waveguide model discussed in the previous section and in Appendix $D$ is used to generate a table of values for $\varepsilon_{\text {eff }}$ at discrete frequencies for a given sensor cell configuration. These values are used in Eq. (8-4) to predict Faraday rotation at those discrete frequencies. In addition, the lead effects for the complete sensor system can be included by using the developments of Appendix B; the responses just described for the travelling wave coil proper are used as $\theta_{3}(\omega)$ in Eq. (B-17). Such a comprehensive model [75] is vsed to predict the behavior of all the travelling wave sensor experimental configurations and to evaluate responses for a broader range of possible sensors.

Figure 10-9 shows model predictions for several different travelling wave sensor example configurations. In the examples, a coaxial transmission cell with methanol as the liquid dielectric is used. All fiber coils have 20 toms, a mean diameter of $3 \mathrm{~cm}$, and a constant helical pitch of $2.4 \mathrm{~cm}$. This fixes the diameter-to-pitch ratio at $\mathrm{d} / \mathrm{x}_{0}=1.25$. Curve (a) in Fig. 10-9 is the relative magnitude of the Faraday rotation for the reference coil having planar turns (zero pitch). The bandwidth for this sensor coil is $48.3 \mathrm{MHz}$. Curve (b) is the sensor response for a travelling wave coil system with $\varepsilon_{\mathrm{r}}=32.6$. The helical fiber coil is assumed self supporting in the liquid mediun, and the methanol is treated as nondispersive. Equation (8-6) describes the response for curve (b), which is very close to having the maximum bandwidth for a travelling wave sensor. The travelling wave condition, Eq. (8-12), for this dielectric is met when $d / x_{0} \approx 1.20$. A very wide bandwidth, $1.34 \mathrm{GHz}$, is predicted for this near-ideal sensor configuration. If liquid dispersion is considered in the model, the Faraday response is given by curve (c) in Fig. 10-9. Absorption by the methanol damps the response at the higher frequencies; the bandwidth is reduced by a factor of 5.5 from that of curve (b). Liquid dispersion also 

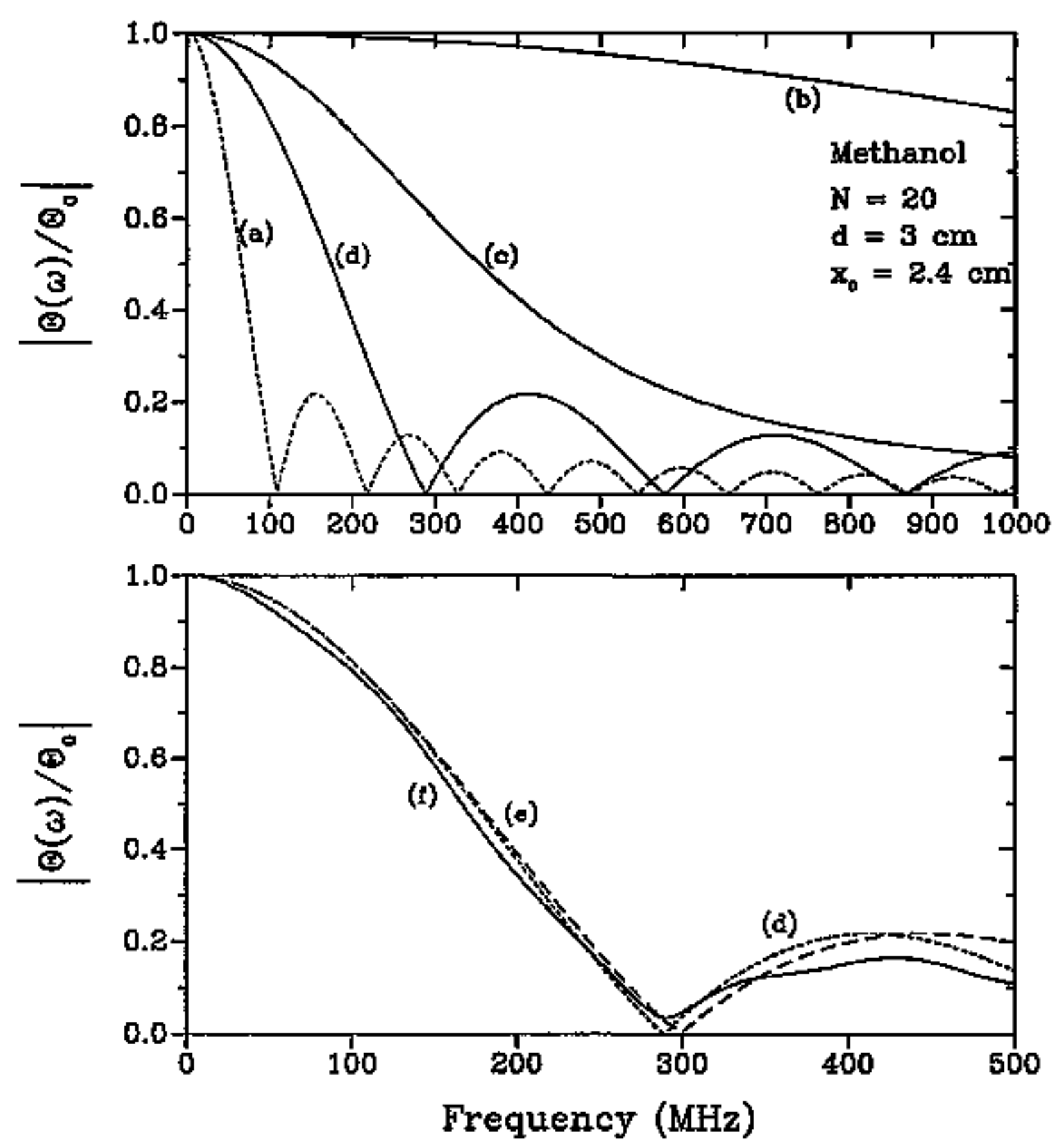

Fig. 10-9. Model predictions of the relative magnitude of the Faraday rotation for a travelling wave fiber current sensor with methanol as the dielectric. (a) Planar coil only; (b) helical coil, no dispersions; (c) helical coil, liquid dispersion only; (d) helical coil, $\varepsilon_{\text {eff }}$ due to the teflon, no dispersions; (e) helical coil, $\varepsilon_{\text {eff }}$ waveguide dispersion only; and (f) helical coil, $\varepsilon_{\text {eff }}$ liguid and waveguide dispersion, and fiber lead effects. 
eliminates the nulls from the sensor response, meaning the magnitude never drops completely to zero. This is an argument for potential utilization of fiber sensors well beyond their $3 \mathrm{~dB}$ bandwidth or the expected cutoff frequency.

In practical travelling wave systems, the presence of the fiber support mandrel was shown to perturb the transmission line relative permittivity. If this effect is considered in our example model, curve (d) in Fig. 10-9 results. In generating this response, a teflon support tube $\left(\varepsilon_{\mathrm{r}}=2.0\right)$ having inner and outer diameters of 2.4 and $3.18 \mathrm{~cm}$ was used, and the coaxial transmission cell had center and outer conductor diameters of 0.52 and $5.75 \mathrm{~cm}$. These dimensions are exactly those used for all the experimental configurations in this study. An effective relative permittivity of 14.07 is computed for the methanol-leflon dielectric system. Once again, the liquid is assumed nondispersive in generating the response curve (d). The $3 \mathrm{~dB}$ bandwidth for this curve, computed using Eq. (8-7), is $127.9 \mathrm{MHz}$.

In the lower half of Fig. 10-9, the perturbations in the Faraday response due to the addition of waveguide dispersion, liquid dispersion and lead effects are shown. Curve (e) adds the waveguide dispersion but treats the liquid medium as lossless. The $\varepsilon_{\text {eff }}$ values from the dotted reference curve in Fig. 10-6 were used to generate this response. Since the effective relative permittivity of the methanol increases with frequency, the phase matching between the electromagnetic signals actually improves as the frequency increases. This leads to a slightly larger bandwidth for curve (e) when compared to curve (d) in Fig. 10-9. Signtificant increases in response are seen in the second lobe near $500 \mathrm{MHz}$. This increased travelling wave performance is even more pronounced for other liquids, such as water, since the waveguide dispersion for the $\mathbf{T M}_{01}$ mode is much greater (see Fig. 10-7).

All travelling wave sensor effects, including the fiber coil lead effects, were considered in generating the response curve (f) in Fig. 10-9. Leads are assumed to have a length defined in Appendix $B$ by $X=R$ and be oriented perpendicular to the planar 
magnetic wave, having no travelling wave characteristics. This lead configuration describes precisely that used in the experimental sensor cells. Both absorption in the methanol and increased propagation time in the fiber leads lower the sensor bandwidth in curve (f) of Fig. 10-9. Lead effects are not as pronounced in this model description since $\mathbf{N}=20$. As might be expected, coils with fewer numbers of turns exhibit further reductions in bandwidth due to the lead effects. Even when accounting for the many effects that spoil the original near-ideal travelting wave condition, the predicted sensor response still shows a bandwidth enhancement of greater than 2.5 compared to the planar coil sensor configuration. And this occurs without a loss in Faraday sensitivity.

In Fig. 10-9, two other features of curve (f) are noted. First, the response never drops to zero. One explanation for this phenomenon, the liquid dispersion effects, was given earlier when describing curve (c). Missing nulls in the response curve can also be created by the fiber leads, since they are arranged in a non-travelling wave configuration. For curve (f) in Fig. 10-9, a combination of both liquid dispersion and lead effects contribute to the non-zero response near $300 \mathrm{MHz}$ and also to the loss in higher frequency response in the second lobe. The second noted feature in carve $(f)$ is the presence of a weak modulation over the entire response. This is entirely due to the fiber leads and appears to have a modalation frequency which very closely follows that for the planar coil shown in corve (a). This resalt is somewhat expected, since the leads are configured in a planar layout and contribute additional length to the coil proper (as described in Appendix B). In travelling wave coil configurations that have fewer turns, the lead effects are more dominant, and the modulation is more pronounced.

The Faraday rotation phase also ondergoes various transfomations as the effects of the liquid travelling wave cell configurations are compounded. Some of these are illustrated in Fig. 10-10. Shown are phase response curves that correspond to the magnitudes in the lower half of Fig. 10-9; the curve letter indicators remain the same. Curve (d) gives the expected response for a sensor whose frequency dependent phase is 


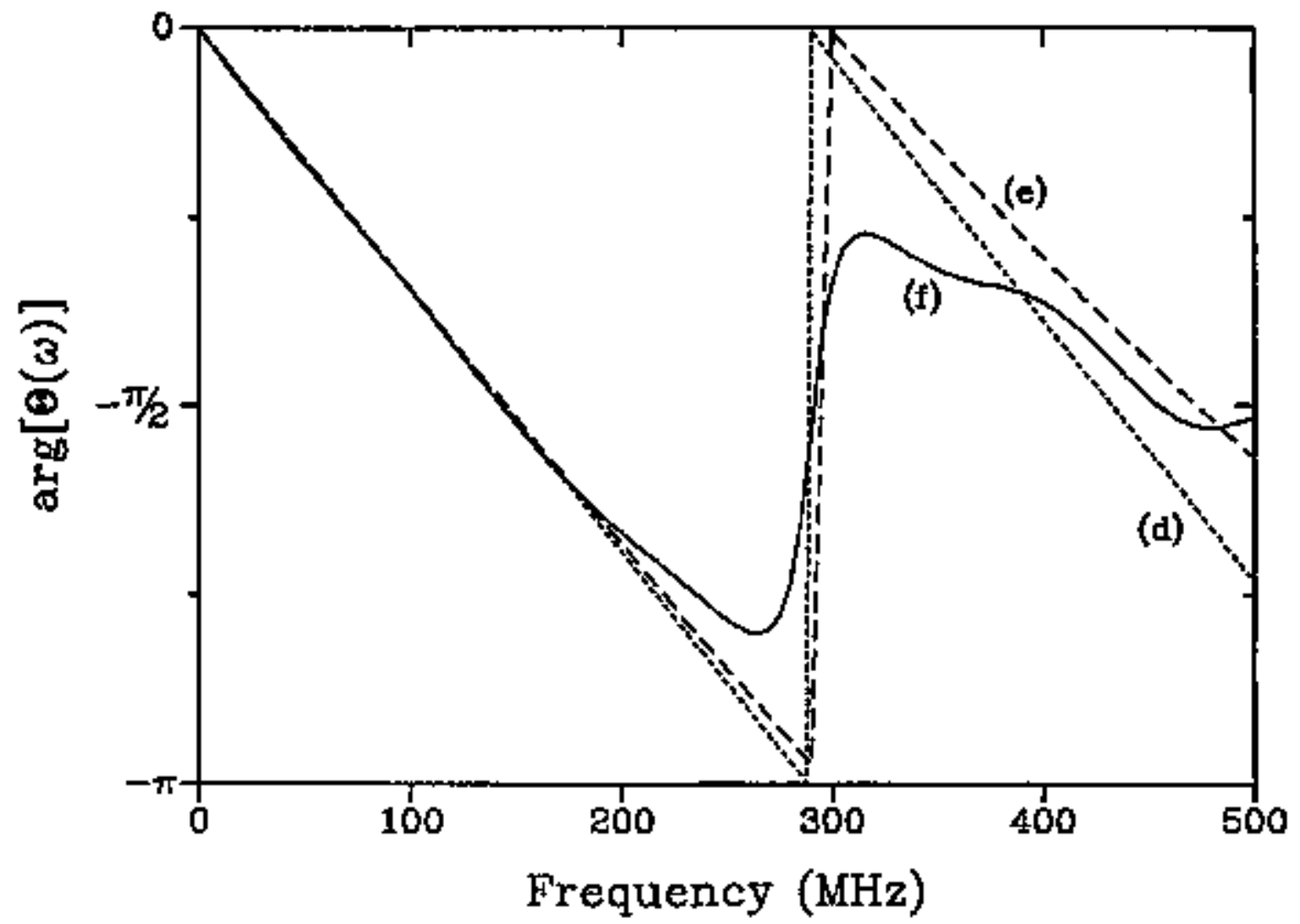

Fig. 10-10. Model predictions of the Faraday rotation phase for a methanol-filled travelling wave current sensor. The sensor parameters and the curve designators correspond to Fig. 10-9.

determined by the total interaction time, $\phi \propto \omega$. The "apparent" interaction time for a travelling wave sensor decreases as the phase matching increases, when in reality, the actual interaction time lengthens. The decrease in interaction time is given by the factor in parenthesis in Eq. (8-5b). In fact, Eq. (8-5b) describes the phase angle (less the sign change) for the travelling wave sensor used to generate curve (d) in Fig. 10-10. Curve (e) shows the increasing phase angle with frequency created by the constantly increasing value of $\varepsilon_{\text {eff }}$ for the lossless methanol model. The phase is a nonlinear function of frequency, giving rise to the less-abrapt phase flip near $300 \mathrm{MHz}$ and the lag in phase angle as freguency increases. 
The most noticeable phase deviations occur in curve (f) in Fig. 10-10. Flattening and diminution of the phase angle curve near the transition freguency is what contributes to the missing nulls in the magnitude curve. From previous discussions of the Faraday response for fiber sensor coils (see Eq. (5-5) and Fig. 5-1), the zero response magnitude occurs at frequencies where the phase is an integer multiple of $-\pi$. For curve $(f)$ in Fig. 10-10, the phase never achieves this value, and thus, the corresponding magnitude in Fig. 10-9 never drops to zero. Also noticeable in the phase curve (f) is the weak modulation due to the fiber coil leads and the increased phase flattening at the highest frequencies doe to absorption in the methanol.

Many of the mechanisms discussed earlier in this chapter, and illustrated in Fig. 10-9, lead to reduced bandwidths for practical travelling wave fiber current sensors. In particular, the waveguide and liquid dispersion in the travelling wave transmission cells and the presence of the non-travelling wave configuration of the fiber coil leads prectude achieving the near-infinite bandwidth enhancement factors described in Chapter 8 for the ideal configurations. These limitations are illustrated in Fig. 10-11 where predicted magnitudes of the bandwidth enhancernent factor are plotted for several configurations of the methanol example model. The diameter of the helical coil is again fixed at $3 \mathrm{~cm}$ and the pitch is varied to allow for a changing $d / x_{0}$ in the plot abscissa. Curve (a) is the BEF magnitude for the nondispersive, travelling wave sensor; one point on this BEF curve comes from curve (b) in Fig. 10-9. The BEF magnitude is generated from Eq. (8-10) with $\varepsilon_{\mathrm{r}}=32.6$. At $d / x_{0}=1.2$, the $B E F$ approaches infinity. If the model now includes liquid dispersion in the methanol, curve (b) in Fig. 10-11 results. For this curve, the BEF is generated directly from $\mathrm{Eq}$. (8-9) with $\triangle \mathrm{B}$ (travelling wave) extracted from numerical computations. The peak BEF in curve (b) is limited to $\sim 5.04$ and occurs at $d / x_{0} \approx 1.23$. Although the maximum-achlevable bandwidth enhancement is significantly reduced due to the absorption, the design parameters for the best travelling wave sensor performance remain essentially the same (peak BEF is approximated by Fig. 8-4). From further 


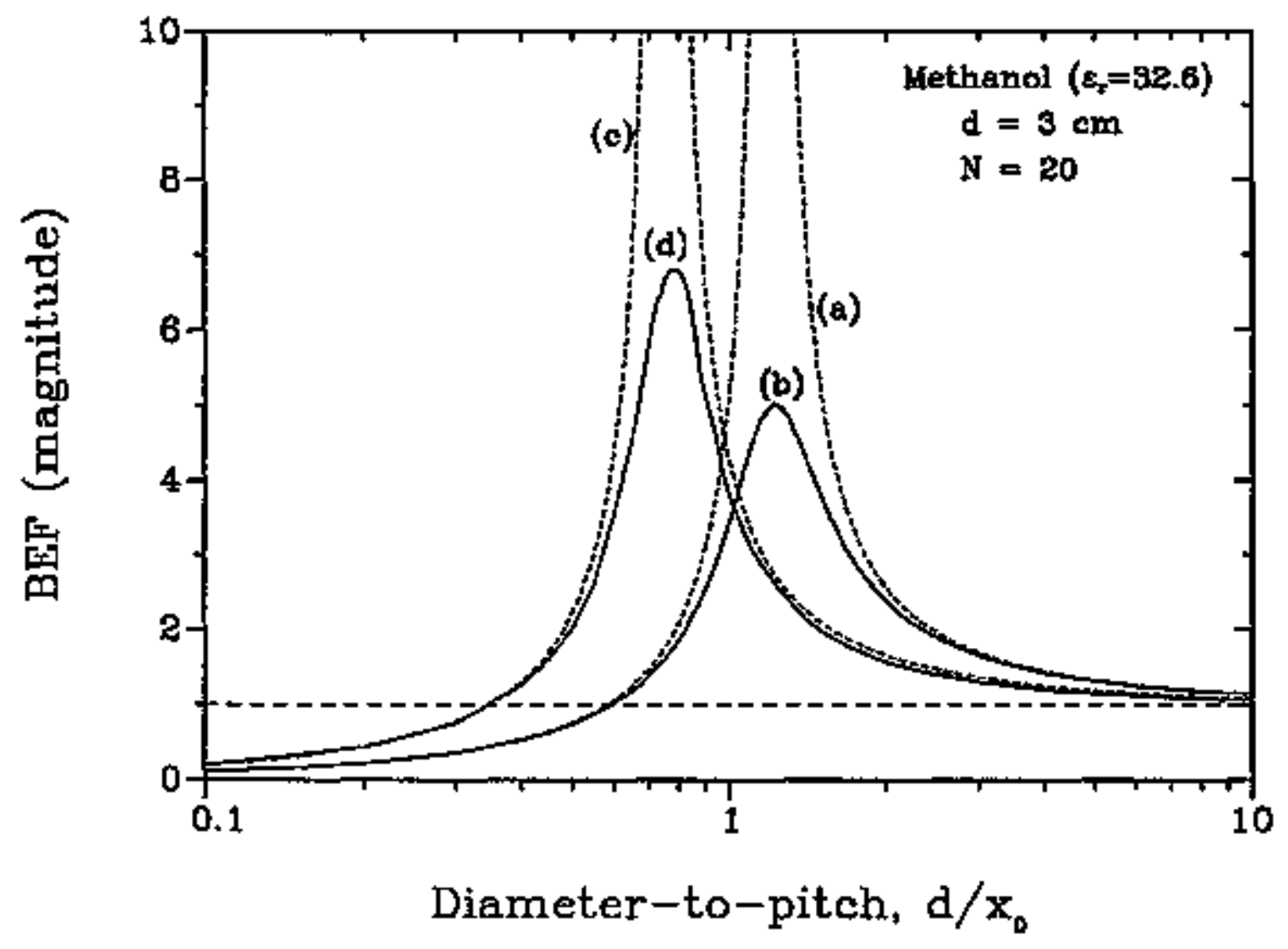

Fig. 10-11. The bandwidth enhancement factor as a function of the diameter-to-pitch ratio for travelling wave current sensors using a methanol dielectric. (a) Helical coil only, $\varepsilon_{\mathrm{r}}=32.6$; (b) helical coil, liquid dispersion only; (c) helical coil only, $\varepsilon_{\text {eff }}=14.07$; and (d) helical coil, $\varepsilon_{\text {eff }}$, all effects included.

investigations using the extended models, it appears that the larger the liquid absorption, the greater the reduction in the maximum BEF. This prediction is not surprising.

Curve (c) in Fig. 10-11 is the BEF magnitude for the travelling wave current sensor including effects produced by the teflon fiber support tube but neglecting dispersion in the methanol medium. The BEF curve, computed using Eq. (8-10), is now shifted to smaller $d / x_{0}$ values to account for the effective relative permittivity, $\varepsilon_{\text {df }}=14.07$. Because of the nondispersive medium, a BEF approaching infinity occurs for $d / x_{0} \approx 0.753$. Curve (d) is generated for the comprehensive travelling wave model 
including all perturbing effects. The peak BEF magnitude is $~ 6.82$ and occurs when $d / x_{0} \approx 0.785$. Again this maximum BEF, though attenuated, appears at nearly the same $d / x_{0}$ value as that for the ideal travelling wave sensor. The peak BEF magnitude for curve (d) is noticeably larger than that for curve (b). This appears to solely depend on the static value of the $\varepsilon_{e d}$ for the transmission cell dielectric medium; the smaller the value of $\varepsilon_{\text {off }}$ the greater the degree of phase matching and the larger the maximnmachievable BEF. The inclusion of lead effects in the model used to generate curve (d) in Fig. 10-11 reduces the peak BEF by only $3 \%$. This small reduction is obviously due to the large number of turns $(\mathrm{N}=20)$ used in the model fiber coil. When fewer turns are used, more pronounced reductions in the maximum BEF are predicted. Also noticeable in curve (d) is the weak modulation effects produced by the fiber coil leads.

The complete extended models describing the liquid travelling wave current sensors predict that large bandwidth enhancements are possible for many configurations. In the previous example for a sensor containing methanol as a dielectric, BEFs greater than 6.0 were predicted. However, the model predicts that BEFs will always be below 10 for most configurations using methanol. For other liguid dielectrics, larger bandwidth enhancements may be possible, but for the three test liquids chosen for experimentation, estimates are that BEFs will remain less than 10 . Bandwidth enhancement is very much dependent on travelling wave sensor design parameters such as transmission cell geometries, fiber support material and geometry, fiber coil pitch and number of tarns, and fiber coil lead length and configaration. The comprehensive models indicate that highly precise estimates of the effective relative permittivity can be made, and that static values for $\varepsilon_{\text {arf }}$ can be used in Eq. (8-12) to find $d x_{0}$ for maximum performance in the travelling wave sensor design. 


\section{CHAPTER 11}

\section{TRA VELLING WAVE SENSOR MEASUREMENTS}

The relative magnitude of the Faraday rotation was measured for several configurations of the travelling wave fiber current sensors. Relative pemittivity was varied through use of air and liquid dielectrics, and diameter-to-pitch ratio was varied in the helical fiber coils. Travelling wave cell configurations are discussed in detail in Chapter 9, and the laboratory experimental instnmentation and hardware are shown in Figs. 4-1 and 9-1. Measurements with the liquid-filled cells were conducted up to a frequency that was limited by loss in response signal-to-noise: a combination of the Faraday rotation bigh frequency rolloff and the absorption of the transmitted power in the liquid dielectric. Thus, maximum experimental freguencies were $625 \mathrm{MHz}$. The transmitted power through the coaxial test cells was held constant for most of the high frequency testing so that monitored currents could be related directly to power. However, as absorption increased it was sometimes necessary to accept the reduced transmitted power and correct the data for the relative magnitudes. Also, because of the large impedance mismatches that occurred for some of the travelling wave test cells, corrections to the estimated conducted currents were attempted. This was only necessary in a few extreme cases.

For all the experimental sensor configurations, an increase was observed in response $3 \mathrm{~dB}$ bandwidth compared to that for an equivalent planar fiber coil. The travelling wave fiber sensor concept was proven, and quantitative information was acquired on sensor parametric effects. Also, the complete extended current sensor models developed in Chapter 10 predicted quite accurately the magnitude versus frequency response curves for most of the systems. Trends in the measured responses were noted for the others. 


\section{Airline Measurements}

One configuration of a travelling wave sensor with an air dielectric was tested. The helical fiber coil parameters were $N=10, d=3 \mathrm{~cm}$, and $d / x_{0}=1,0$. Figare 11-1 shows the Faraday response for that system. Plotted are the measured values of the relative magnitude, shown as the open circles, and the model predictions, the solid curve. Scatter in the measored data is approximately $1 \mathrm{~dB}$ around the plotted points. The measured $3 \mathrm{~dB}$ bandwidth for the sensor system is $-109.5 \mathrm{MHz}$.

As seen in the Fig. 11-1, agreement is excellent between the experimental and theoretical values. Below $350 \mathrm{MHz}$, little deviation is observed; however, at the higher frequencies some loss in measured response is seen compared to that predicted by the

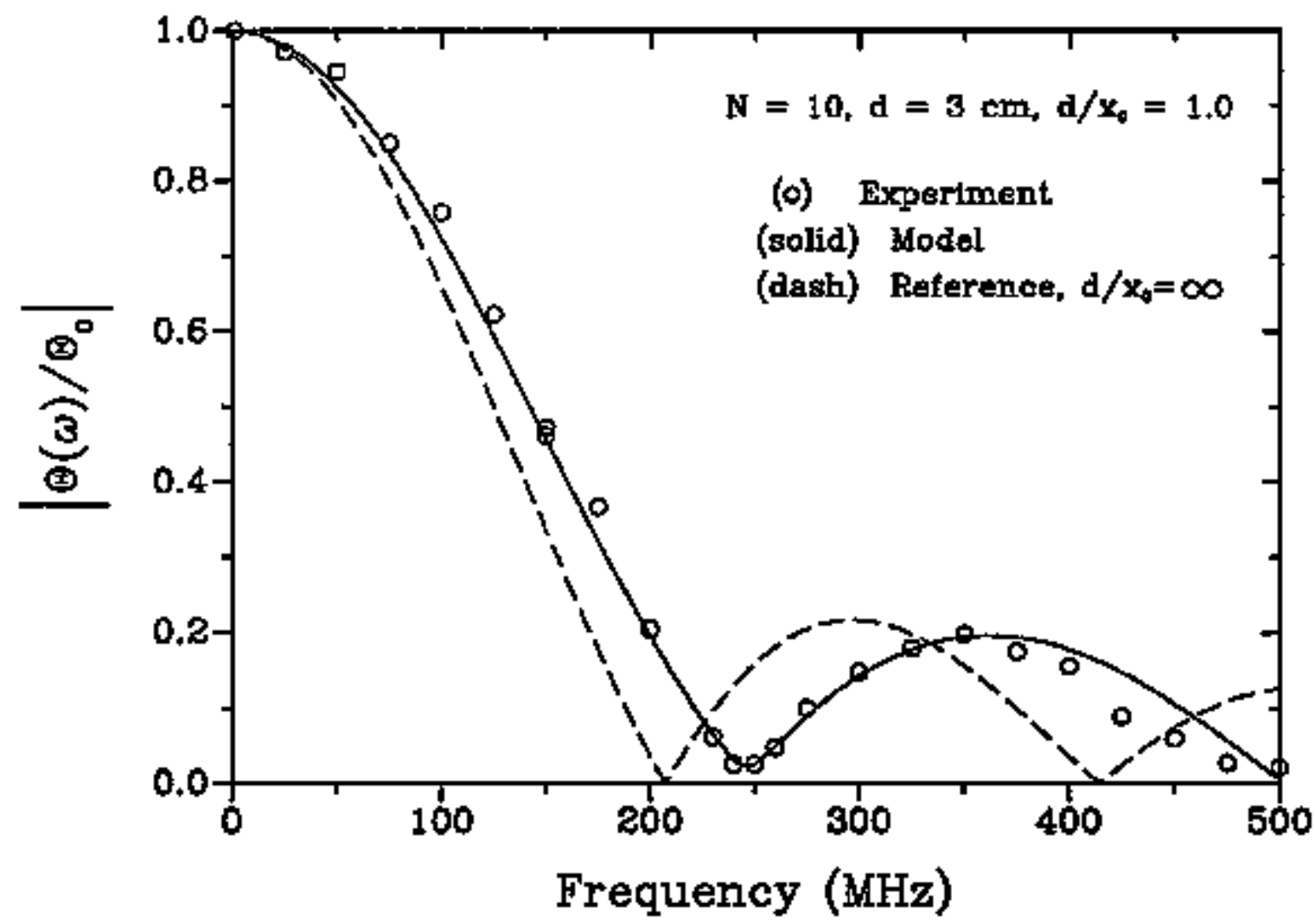

Fig. 11-1. The relative magnitude of the Faraday rotation for a coaxial airline travelling wave fiber current sensor. 
model. This high freguency discrepancy between model and experiment is consistent throughout all measurements made for the travelling wave sensors, air or liquid cells, and can only be explained by a deficiency in the model, nnaccounted perturbations in the EM properties of the transmission ceils, or a combination of the two. The model predicts quite accurately the non-zero response minimum near $245 \mathrm{MHz}$ in Fig. 11-1. This missing null is caused by the fiber leads that enter and exit the coaxial line in a nontravelling wave configutation. The fiber leads are also configured such that they add an equivalent half torn to the fiber coil length (see Appendix $B$ for a further explanation), which reduces the sensor response bandwidth.

Also plotted in Fig. 11.1 is a dashed reference curve for the equivalent planar coil fiber current sensor. This reference sensor also includes fiber leads in the exact configuration as those for the travelling wave coil. The measured values for the travelling sensor show an enhanced high frequency response when compared to that of the reference sensor. The bandwioth enhancement factor (BEF), determined from the ratio of interpolated $\Delta \mathrm{B}$ values, is approximately 1.19 ( $\Delta \mathrm{B}$ for the reference sensor is $\sim 103.4 \mathrm{MHz}$ ). This is very close to 1.2 , the BEF predicted for $\varepsilon_{\mathrm{r}}=1$ and $\mathrm{d} / \mathrm{x}_{0}=1$ by the ideal travelling wave theory presented in Chapter 8. Thus, well within experimental error, the enhanced response of this sensor can be detemnined from simple theory.

Judging from the predictions shown in Fig. 11-1, the extended models (that account for fiber lead effects) can be used to identify optinum design parameters for general configurations of the arline travelling wave fiber sensor. If the fiber coil parameters $\mathrm{N}=10$ and $\mathrm{d}=3 \mathrm{~cm}$ are used in the model and the coil pitch is allowed to vary, the BEF attains a peak value of $\sim 1.37$ at $d / x_{0}=0.33$. This prediction is essentially identical to that found from Eg. (8-10) and shown in Fig. 8-3 for the idealized travelling wave sensors. The mathematical developments leading to Eq. $(8-10)$ neglect the presence of the fiber leads, both for the planar as well as the travelling wave coil. Obviously, the lead configuration in a 10 tam airline travelling wave coil has little, if 
any, effect on the relative bandwidth enhancement. However, it is expected that for coils with fewer turns or for systems with larger high frequency enhancements that lead interactions may be more significant, producing somewhat reduced BEFs.

Regardless, it is obvious from these predictions and the validating measurements on the air dielectric travelling wave current sensor that significant increases in sensing bandwidth cannot be achieved with only the helical fiber structure. Slow-wave mechanisms for improved ghase matching are required. In the present travelling wave cell design, this entails use of higher relative permittivity dielectrics.

\section{Liguid Cell Measurements}

In addition to the three test liquids nsed as dielectrics in the coaxial transmission cells, three different fiber coil helical pitches were employed. These were $x_{0}=3.0,2.0$, and $1.5 \mathrm{~cm}$, which correspond to $\mathrm{d} / \mathrm{x}_{0}$ values of $1.0,1.5$, and 2.0 , respectively, since the mean coil diameter was held constant at $d=3 \mathrm{crn}$. The number of fiber tums was 20 for the coils with $d / x_{0}=1.5$ and 2.0 , and 14 for the coil with $d / x_{0}=1,0$. This difference was deemed necessary due to the total length of the cell working volume, approximately 46 $\mathrm{cm}$. A 20 tum coil with the largest coil pitch would have been $60 \mathrm{~cm}$ in length. Is all, nine different sensing configurations were available for experimentation. Actually a few additional configurations were explored, involving a varied cell center conductor diameter. These variations caused cell impedance to differ from $50 \Omega$ and prodnced deviations in the oielectric effective relative permittivity (see Eqs. (10-2) and (10-3)), but changes in the measured Faraday responses for the sensors could not be distingrished due to the relatively large experimental errors $(\sim 1 \mathrm{~dB})$. For this reason, the responses presented for the travelling wave current sensors are identified only by test liquid, the fiber coil $d / x_{0}$, and the number of coil turns.

A typical response curve showing the relative magnitude of the Faraday rotation versus frequency is presented in Fig. 11-2 [60]. These data were generated from a 


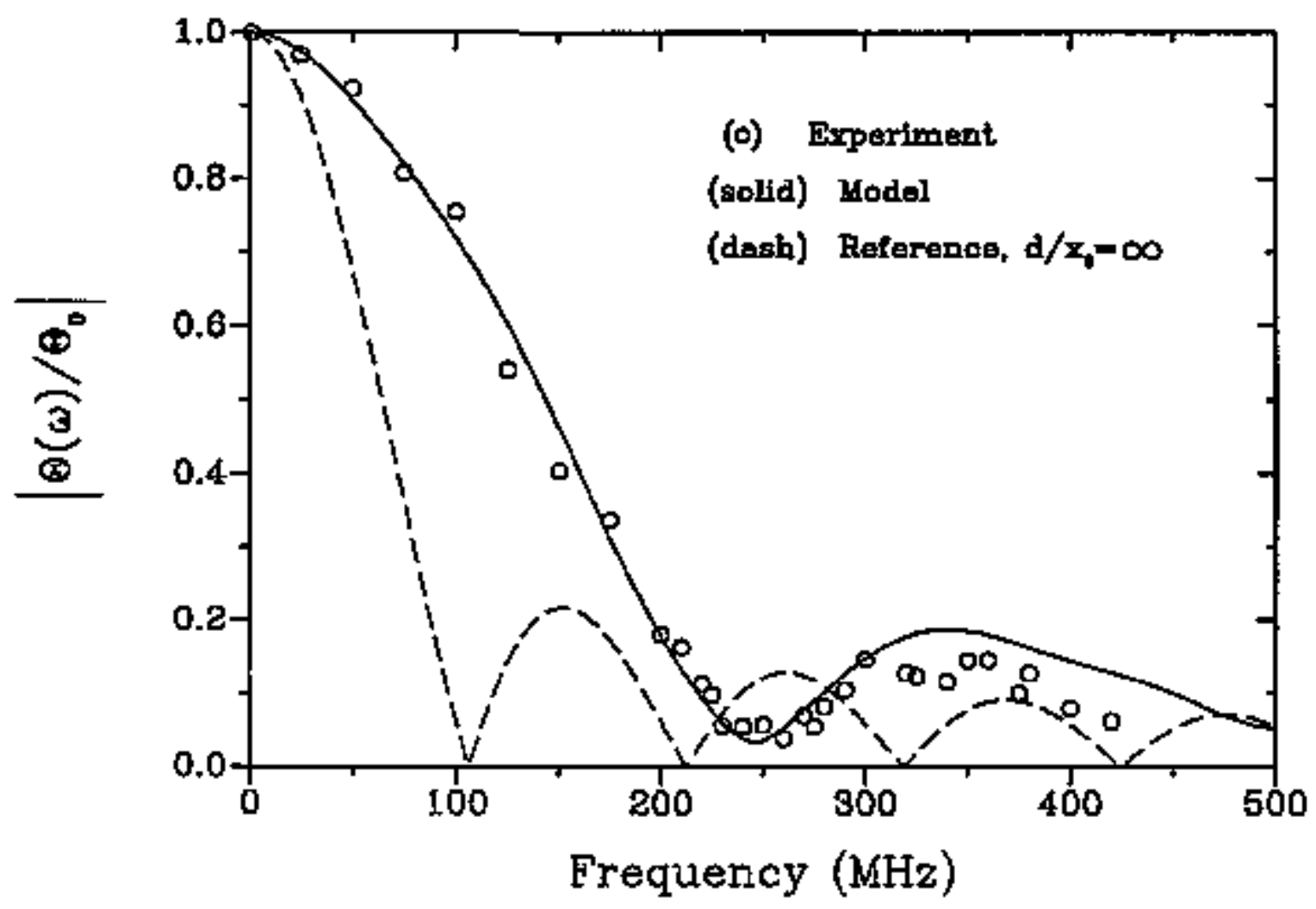

Fig. 11-2. The experimental and theoretical Faraday response for a liguid travelling wave fiber current sensor containing a methanol dielectric. The helical coil has 20 tutns, a $3 \mathrm{~cm}$ diameter and a diameter-to-pitch ratio of 1.5 .

system that used methanol as the cell dielectric and a fiber sensor coil of 20 turns and $d / x_{0}=1.5$. The measured response values (open circles) agree well with the predictions of the sensor model. Deviations between experiment and theory occur only at the higher frequencies, in the second response magnitude lobe. Explanations for this discrepancy are offered in the previous section. Both the measured and predicted response corves show the missing noll near $250 \mathrm{MHz}$ in Fig. 11-2 which is the result of liquid dielectric dispersion and fiber coil lead effects.

This travelling wave sensor has a $3 \mathrm{~dB}$ bandwidth of approximately $103 \mathrm{MHz}$ and a BEF of -2.2 when compared to the planar sensing coil (dashed response curve in Fig. 11-2). This enbancement is slightly smaller than the BEF of 2.47 predicted by Eq. (8-10) 
for a nondispersive dielectric with the effective relative permittivity of the methanol/teflon combination and for a leadless helical coil in the same test configuration. The difference in these two BEFs, $11 \%$, is approximately the same as that illusitated for curves (c) and (d) in Fig. 10-11 when $d / x_{0}=1.5$.

Using a liguid as the dielectric medium in the travelling wave fiber sensor systems provides the slow-wave mechanism needed to enhance high frequency Faraday response. Experimental measurements verified the theoretical predictions that increasing the liquid relative permittivity progressively increases the sensor bandwidth. This point is illustrated in Fig. 11-3, where response curves for each of the test liquids are plotted.

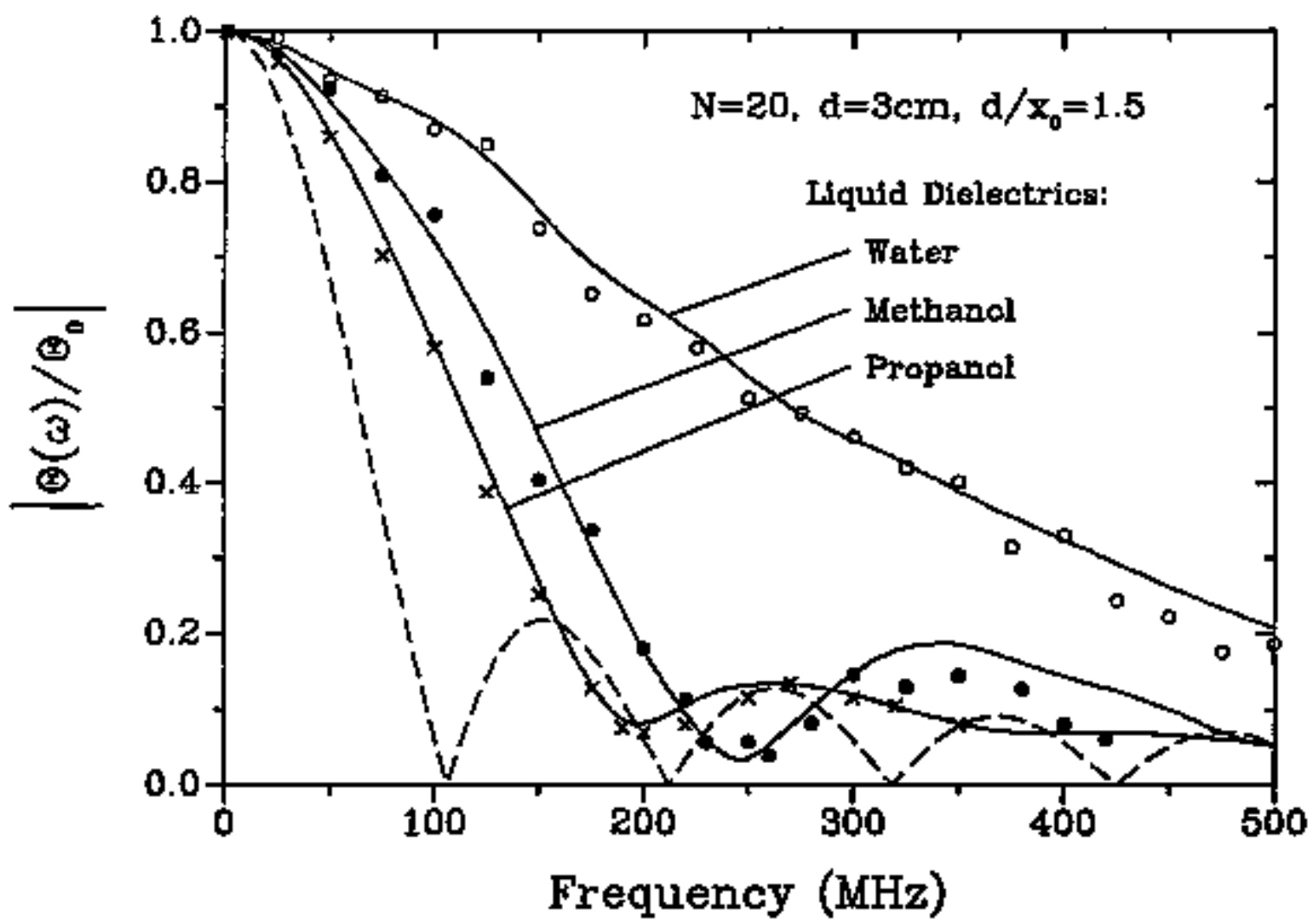

Fig. 11-3. A comparison of the relative Faraday rotation for each of the three test liquids used with the same helical coil. The plotted points are experimental values, and the solid curves are model predictions of response. The dashed curve is the response for an equivalent 20-turn coil with $\mathrm{d} / \mathrm{x}_{0}=\infty$. 
The same fiber sensing coil, $N=20$ and $d / x_{0}=1.5$, is used with the three liquids. Effective relative permittivities (static values) for the liquids increase from 10.35 for the propanol, to 14.07 for the methanol, to 20.94 for the water. Sensor bandwidths are approximately $77.4 \mathrm{MHz}, 103 \mathrm{MHz}$, and $159.5 \mathrm{MHz}$, respectively, for the curves using the three liquids. The corresponding bandwidth enhancement factors are 1.65, 2.2, and 3.4 when compared to the reference curve bandwidth of $46.9 \mathrm{MHz}$. In Fig. 11-3, both measured data (the plotted points) and model-generated responses (the solid curves) are displayed. For the parameters used in these experimental configurations, the model predictions are shown to be highly accurate.

The other travelling wave sensor parameter that affects the bandwidth enhancement factor ts the fiber coil diameter-to-pitch ratio. Experimentation on similar configurations with each test liquid while varying only $d / x_{0}$ showed that increasing the coil pitch improved sensor phase matching and increased response bandwidth. In Fig. 11-4, a plot of the relative magnitude of the Faraday rotation versus frequency is given for several methanol liquid celt configurations. Both experimental values and modelgenerated curves are again plotted for the Faraday response, except for $d / x_{0}=1.0$, which is represented only by a modeling prediction. As discossed earlier, the fiber coil with $\mathrm{d} / \mathrm{x}_{0}=1.0$ had only 14 turns, while the other coils had 20 torns. Since the comprehensive model predictions for the travelling wave fiber current sensors agree quite well with the measured values, liberty was taken to generate the extra curve for the larger coil pitch and appropriately complete the illustrated comparisons. In Fig. 11-4, as $\mathrm{d} / \mathrm{x}_{0}$ decreases ( $\mathrm{x}_{0}$ increasing), the BEF increases. Values of approximately 1.6 (with $\Delta \mathrm{B} \approx 75.3 \mathrm{MHz}$ ), $2.2(\Delta \mathrm{B} \approx 103.5 \mathrm{MHz})$, and $3.9(\Delta \mathrm{B} \approx 183.3 \mathrm{MHz})$ are attained for $\mathrm{d} / \mathrm{x}_{0}=2.0 .1 .5$ and 1.0, respectively. As illustrated previously in Fig. 10-I1 for a similar methanol-filled sensor cell, the BEF grows nonlinearly with decreasing $d / x_{0}$. When $d / x_{0}=1,0$, the sensor system parameters are very close to meeting the travelling wave condition for maximum phase matching. 


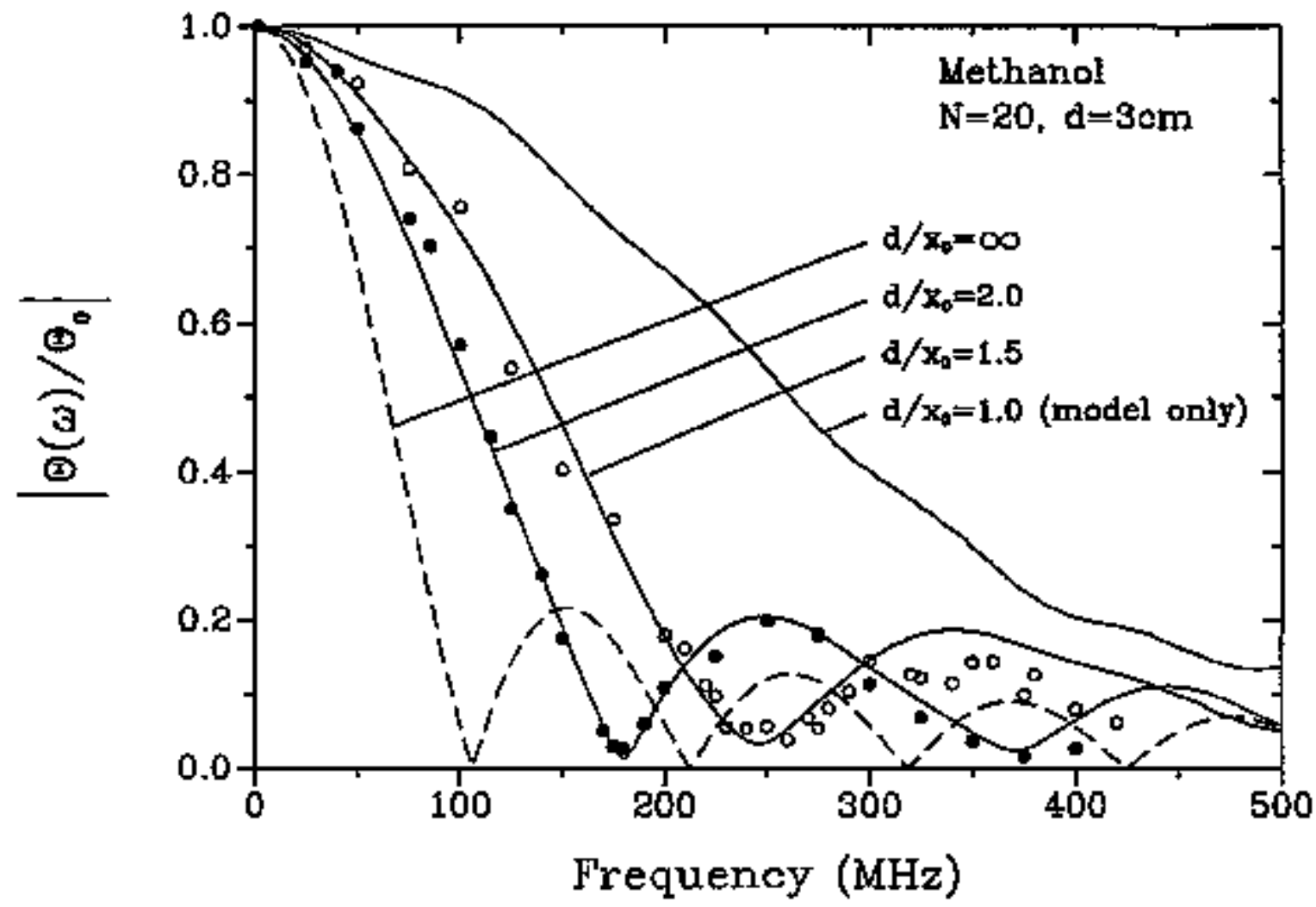

Fig. 11-4. The relative magnitude of the Faraday rotation for a travelling wave current sensor with a 20-turn helical coil in a methanol-filled transmission cell. The diameter-topitch ratio is varied to show the conelation with response bandwidth.

The largest bandwidth enhancement achieved daring experimentation with the liquid travelling wave sensors was approximately 4.1 for a water-filled test cell using a fiber coil with 14 turns and $d / x_{0}=1.0$ [60]. Figure 11-5 shows the Faraday response curve for this sensor. The measured sensor bandwidth is $\sim 300 \mathrm{MHz}$, which is the largest for any multiturn fiber current sensor reported to dafe. An estimate of the systern. sensitivity-bandwidth product (SBP) for this wideband sensor is $1.85 \times 10^{-4} \mathrm{rad} / \mathrm{A}$-s or $1.06 \mathrm{MHz}-\%$ A. This SBP, though much larger than any for a fiber current sensor, is approximately seven times smaller than reported for a bulk optc current sensor using Ga:YIG as the Faraday rotator material [30]. In Fig. 11-5, the rolloff in the response for frequencies above the $3 \mathrm{~dB}$ point is quite dramatic, bot significant response $\mathrm{S} / \mathrm{N}$ is seen 
for frequencies as high as $400 \mathrm{MHz}$. For some current monitoring applications, this sensor could be used to these higher frequencies.

At the low frequencies in Fig. 11-5, below $200 \mathrm{MHz}$, the measured values (open circles) agree well with the model predictions (solid curve). Above $200 \mathrm{MHz}$, however, the experimental and theoretical values deviate significantly. Here, the quantitative ability of the comprehensive travelling wave model to predict sensor responses breaks down. It is postulated that the electromagnetic properties of the transmission cells are not adequately described in the model since contributions that do not adhere to the grasi-TEM assumption are ignored. The source of such contributions conld be large cell

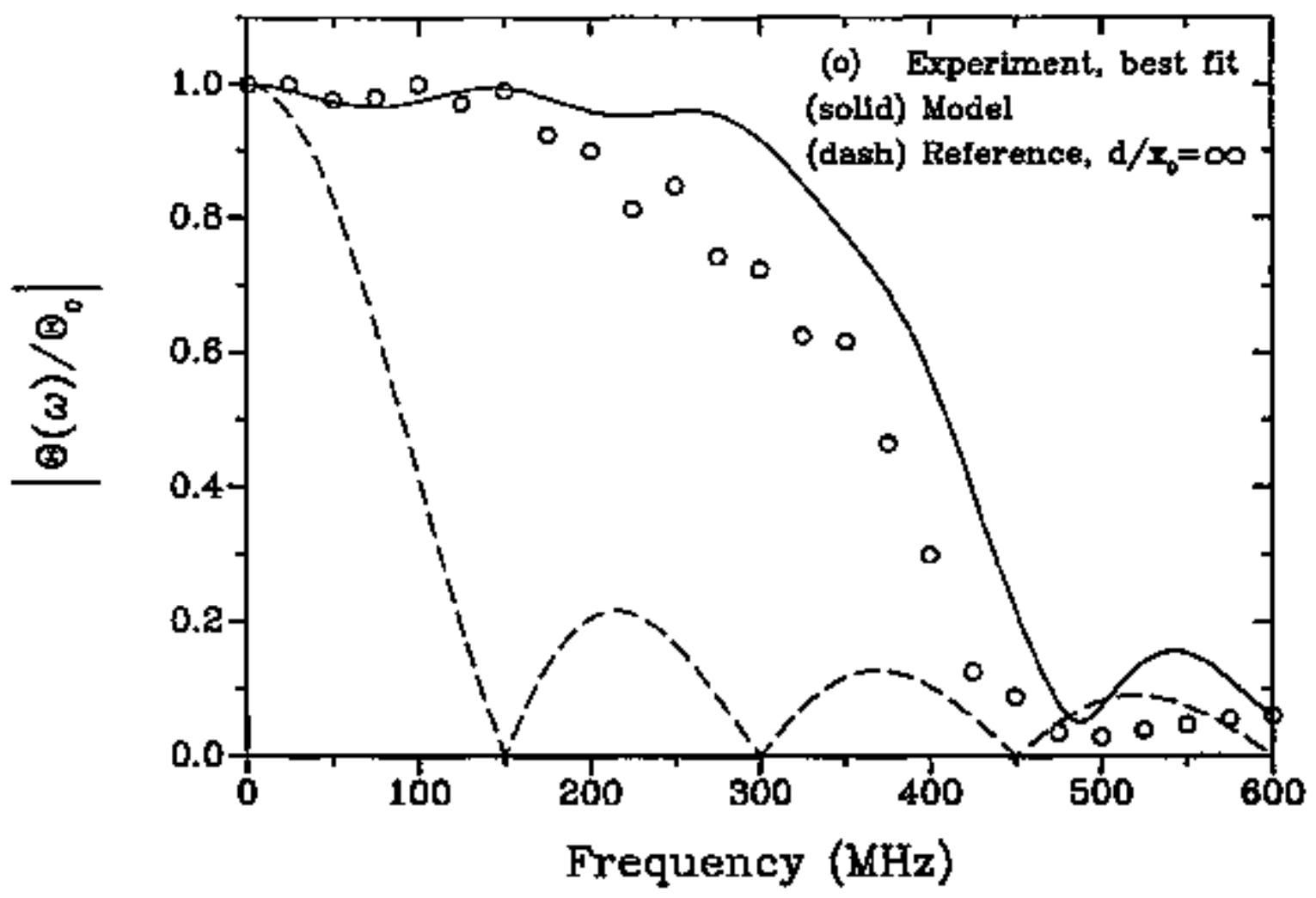

Fig. 11-5. The measured and modeled responses for a travelling wave fiber corrent sensor using a coil with $N=14, d=3 \mathrm{~cm}$, and $d / x_{0}=1.0$ inside a cell containing water. The measured bandwidth for this configuration is approximately $300 \mathrm{MHz}$, the largest for any of the experimental systems. 
reflections, cell multimoding by nonazimuthally-symmetric modes, or a combination of these and other high frequency effects.

In spite of these discrepancies, gualitative trends are observed in the measured response values that are predicted by the model. One of these is the abrupt rolloff of the curves. For the model-generated response, this occars between 300 and $500 \mathrm{MHz}$, with magnitudes dropping from approximately 0.95 to near 0.05 . A probable explanation for this fast rolloff lies in the rapid increase of the effective relative permittivity for the $\mathrm{TM}_{01}$ mode in the water transmission cell. From Fig. 10-7, the value of $\varepsilon_{\text {eff }}$ increases from -23 at $200 \mathrm{MHz}$ to -40 at $500 \mathrm{MHz}$; thus, at the higher frequencies, the magnetic wave is travelling much slower. For freguencies below $\sim 150 \mathrm{MHz}$, the wave phase velocity is essentially constant and the Faraday response curve will behave normally with a given travelling wave enhancement. But as $\varepsilon_{\text {efr }}$ increases rapidly above $200 \mathrm{MHz}$, the phase matching is also modifted, first increasing slightly, then decreasing significantly as the light in the fiber now begins to outrun the progressively slowing magnetic wave. The travelling wave condition for this water cell configuration should occur when $\varepsilon_{\text {eff }} \approx 23.5$ according to Eq. (8-12). Thus, at approximately $220 \mathrm{MHz}$, the phase matching predicted by the model is gteatest, and the travelling wave enhancement peaks. As the frequency increases beyond $220 \mathrm{MHz}$, the enhancement decreases and the response curve quickly drops. At $500 \mathrm{MHz}$, predictions indicate that travelling wave enhancement has been refuced by nearly a factor of three from its peak value.

The experimentalty determined values in Fig. 11-5 show a rapid rolloff at mpch lower frequencies than predicted by the model. This decreased response at a lower frequency could be caused by the changing wave impedance of the $T M_{01}$ mode in the transmission line. As seen in Fig. 10-8, the wave impedance for the water-filled cells increases significantly, and rapidly, above $150 \mathrm{MHz}$ Thus, the propagating wave becomes less quasi-TEM with a larger field component along the positive axial direction of the cell. This should produce an enhanced phase matching between the magnetic 
wave and the light in the fiber at lower frequencies than predicted by the model (the model does not address the "non-quasi TEM" conditions of the EM wave). A resultant loss in travelling wave enhancement then occurs at somewhat lower frequencies due to the combination of the rapidly increasing $\varepsilon_{\text {eff }}$ and $n$. The experimental values in Fig. 115 appear to confirm this hypothesis, with essentially flat frequency response below 150 MHz, and rapid decrease above that frequency.

Also seen in Fig. 11.5 is the weak modulation created by the fiber coil lead effects. This modulation is apparent in both the measured and model-generated curves. The depth of modulation is slightly larger for this sensing configuration, primarily due to lead effects having a greater impact on coils with snaller numbers of turns. The largest response perturbations caused by lead effects have also been observed when liguid absotption is minimum and when travelling wave enhancements are near maximum. Both of these conditions apply to this water dielectric current sensor configuration.

The largest sensor bandwidths measured for the propanol and methanol-filled travelling wave cells also occurred for the fiber coils with $N=14$ and $d / x_{0}=1.0$. The experimental $3 \mathrm{~dB}$ bandwidths for these sensors were approximately $137 \mathrm{MHz}$ and 221 $\mathrm{MHz}$ for propanol and methanol liquid dielectrics, respectively. Corresponding BEFs are 2.1 and 3.3 for the given configuration. Figare 11-6 is a plot of the Faraday response curves for the widest bandwidth current sensors using each of the three test liquids. As expected, the bandwidth becomes progressively larger as the liquid relative permittivity increases. But at the higher frequencies, the curve for the water-filled cell has a faster response rolloff and crosses the curve for the methanol cell. This indicates that a travelling wave current sensor using methanol as the dielectric medium may be more nseful at the higher freguencies, since the $\mathrm{S} / \mathrm{N}$ remains quite large to well beyond 500 MHz.

In Fig. 11-6 only the measured values for the three liquid dielectric cells are plotted. For these wide bandwidth sensors, the discrepancies between the measured 


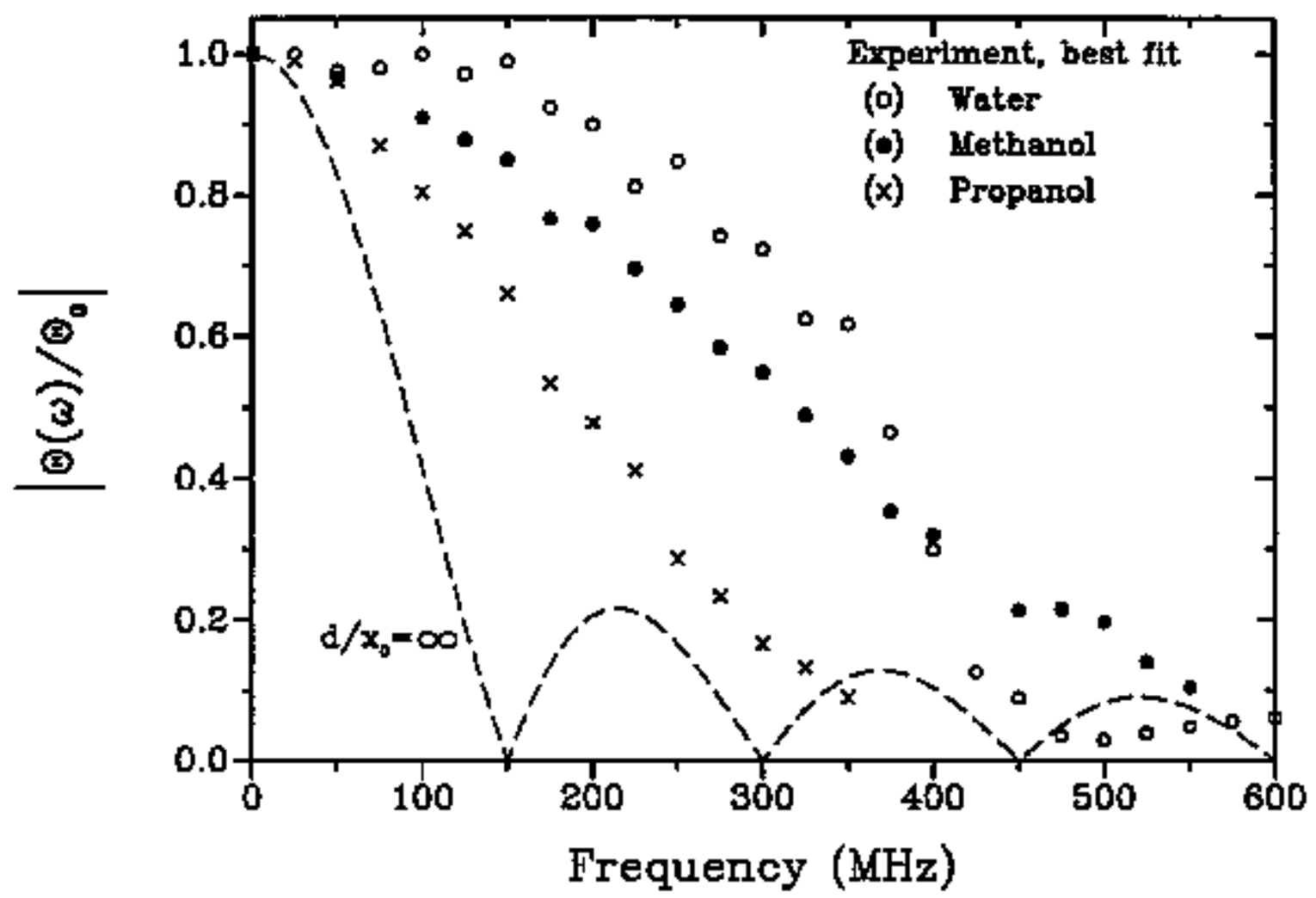

Fig. 11-6. The measured relative magnitude of the Faraday rotation for the largest bandwidth travelling wave sensors using each of the three test liquids. The common coil configuration had $N=14, d=3 \mathrm{~cm}$, and $d / x_{0}=1.0$.

Faraday responses and the model predictions are noticeable, as seen in Fig. 11-5. It is observed that for sensor configurations with low values of the BEF, the comprehensive travelling wave model predicts quite accurately most featores of the response magnitude (as seen in Fig. 11-2); however, for configurations with larger BEFs, the model predictions consistently show a greater relative magnifude at the higher frequencies than the measured values. This occurred for all the liquid cell configurations when $d / x_{0}=1.0$ and $\mathrm{N}=14$. Although, the theory-experiment discrepancy is much less for the propanolfilted sensor cell (approximately $6.5 \%$ at the $3 \mathrm{~dB}$ point in the response curve) and the methanol-filled cell (approximately 11\%) than for the water-filled cell. These discrepancies become sightly greater at the higher frequencies. 


\section{Phase Considerations}

Throughout experimentation with the travelling wave fiber current sensors, only response magnitudes were measured. A phase delay measurement is much more difficult to make than a magnitude measurement, and none was attempted as part of this study. However, this characteristic of the sensor systems is needed to provide the complete transfer function for the Faraday interaction. It is hoped that measurements of the Faraday response magnitude presented here along with the well-developed models to predict sensor behavior is adequate to fully describe the wideband transient response. For most of the liquid-cell experimental sensor configurations, the comprehensive travelling wave model was quite accurate in its predictions of the magnitude; it is reasonable to expect that phase delays can be predicted equally as well. Thus, during travelling wave current sensor design, the models presented in Chapter 10 should be usefol in describing both Faraday rotation magnitude and phase. Time domain response measurements should follow to finalize and verify sensor characteristics. 


\section{CHAPTER 12}

\section{OTHER TRAVELLING WAVE CONFIGURATIONS}

The liquid travelling wave configurations show enhancements in the bandwidth of Faraday effect fiber corrent sensors, all without a loss in the sensitivity. Significant increases in sensor bandwidth and sensitivity-bandwidth product have been demonstrated. However, estimates of the maximum BEFs using models of the liquid transmission line sensing structure are limited to less than 10. Further increases in current sensor bandwidth and sensitivity might be possible, bot significant deviations from the existing travelling wave sinuctures will most likely be required. In this chapter, several innovative schemes that utilize travelling wave current sensing configurations are discussed. Predictions of the potential response, bandwidths and high frequency characteristics, are made where appropriate.

In the liquid travelling wave sensors, dielectric dispersion is the major bandwidthlimiting factor, while the need to support the fiber helix in the ligvid leads to a waveguide dispersion that can also affect higher frequency response. One way of eliminating these factors is to use a solid dielectric as the propagation medium in the slow-wave structure. A very low-loss material at microwave frequencies can be identiffed so that dielectric dispersion is minimized, and the dielectric alone should be capable of supporting the fiber coil without use of other interfering materials. As part of this study, a travelling wave structure using a ceramic dielectric was designed, and its electromagnetic properties were characterized.

Another disadvantage of the liquid (and air) travelling wave current sensors is size. The liquid test cells were quite large, $6.35 \mathrm{~cm}$ diameter and $53.8 \mathrm{~cm}$ length, in order to accommodate the 20 tum fiber helical coils. Such large size is tolerable for laboratory development, but practical systems need to be much smaller. Reductions in device size can be accomplished in three ways: (1) using dielectric materials with 
extremely large relative permittivities, so that $x_{0}$ is mininized for a given bandwidth; (2) decreasing the fiber coil diameter so high-sensitivity coils with many turns are still short; and (3) developing optical fibers with larger Verdet constant materials so high sensitivity is achieved with fewer coil turns. All of these methods have limitations, some of them severe, due to present technology.

Thus, constructing usable travelting wave current sensors will require new technological developments and possibly new materials in new configorations. Fiber coils have been utilized exclusively because of their ease of use, but they may have to give way to other optical waveguide structures. Or the coaxial transmission line with helical light guide must be abandoned altogether. New configurations are postulated here in hopes of stimulating further development of wideband optical current sensors. In addition to the ceramic dielectric transmission line structure, devices that use helical optical waveguides, planar integrated waveguides, and bulk optical materials are discussed. Travelling wave configurations include the use of helical conductor paths as well as looping optical paths. For the sake of completeness, travelling wave designs that utilize combinations of the fiber sensing technigues discossed in the earlier chapters are covered here. The theoretical framework derived for those technignes provides an excellent means for predicting high frequency response, even in non-fiber systems.

\section{Ceramic.Dielectric Travelling Wave Sensor}

A solid material with a large relative permittivity that is suitable for use in a transmission cell is difficult to find. Typically, polyethylene or teflon is used in coaxial cables because of their flexibility and good microwave characteristics; but they have relative permittivities of less than 2.5 . Higher $\varepsilon_{r}$ materials are generally limited to the high-alumina content ceramics and the titanates (barium, strontium, calcium, magnesinm and lead) [67]. These materials have very good microwave characteristics, but lack much of the fabrication flexibility afforded by the polyethylene or tefion. With these 
properties in mind, a diamonite high alumina (95\%) ceramic with a static relative permittivity of 9.6 was chosen as the candidate material for a solid dielectric travelling wave structure. This ceramic does exhibit some dielectric dispersion, as $\varepsilon_{\mathrm{r}}$ drops to 9.36 at $10 \mathrm{GHz}$

The fabricated coaxial transmission cell with ceramic dielectric is shown in the cross- sectional composite view of Fig. 12-1 [34]. The test cell working volume consists of five ceramic pieces; each were cast and fired separately. The core component is a tube $6 \mathrm{~cm}$ long with a $1.3 \mathrm{~mm}$ inner diameter and $6 \mathrm{~mm}$ outer diameter. Along the outer surface of the tube a 10-turn helical groove is machined approxinately $0.75 \mathrm{~mm}$ deep. Mating ceramic parts were fabricated so the entire dielectric structure filled the coaxial cell working volnme: $10 \mathrm{~cm}$ long and $1.59 \mathrm{~cm}$ in diameter.

The ceramic pieces were designed to double as an annealing fixtore for the 10 turn singlemode fiber coil. Fiber annealing is necessary on this sensor in order to remove the large linear birefringence produced by the small radius turns [21]. Initially,

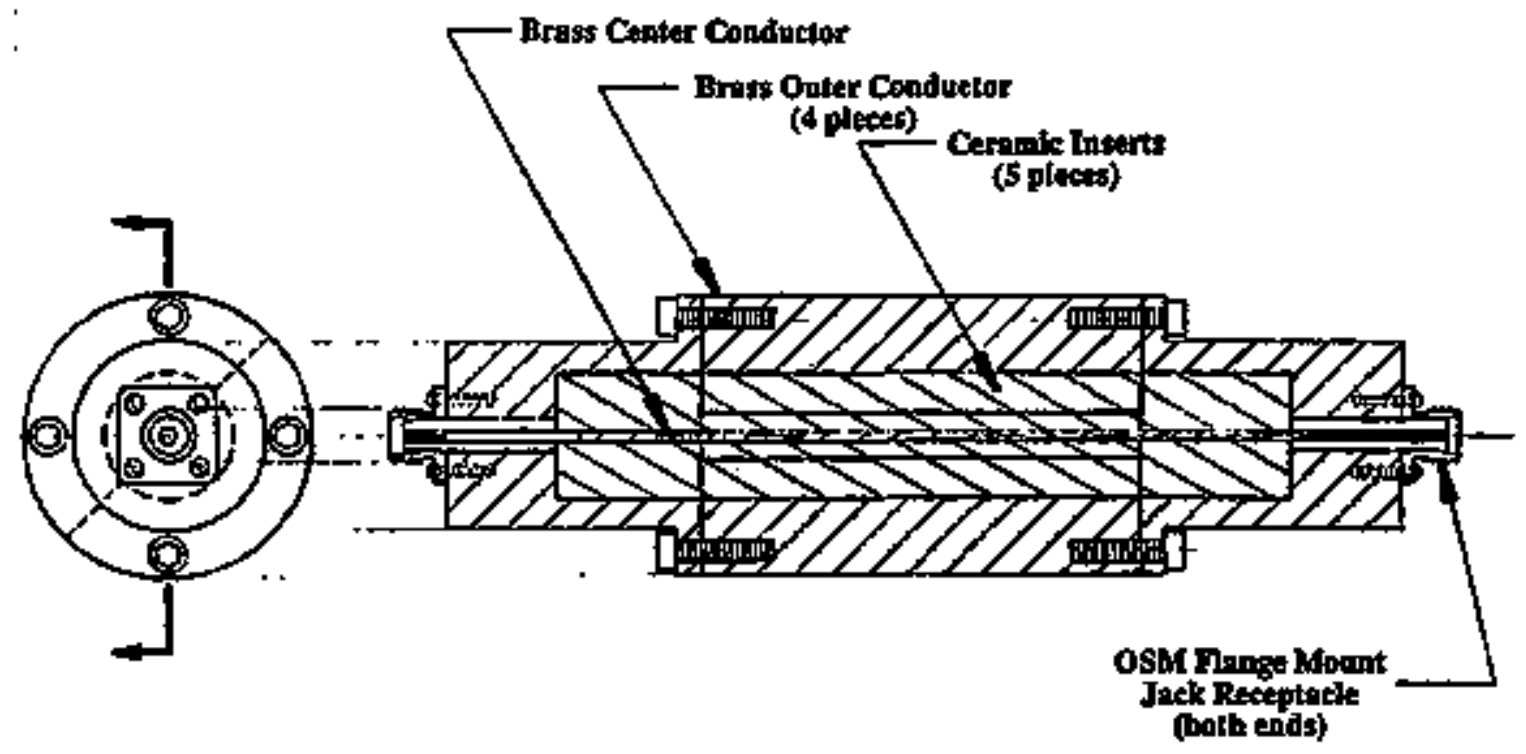

Flg. 12-1. A composite drawing of a travelling wave sensor transmission cell with a ceramic dielectric. 
the fiber is wound into the groove of the tube with ample lengths of excess fiber for leads. The remaining cerarnic pieces are assernbled around the fiber, the transmission line's brass center conductor is passed through the ceramic parts, and the whole stracture is taken to a high temperahure $\left(-850^{\circ} \mathrm{C}\right)$ to perform the annealing process [22]. After annealing, the outer conductive shield of the transmission cell is assembled around the dielectric containing the fiber coil. Cotaway and disassembled views of the travelling wave transmission cell (less the optical fiber) are shown in the photographs of Fig. 12-2.

Annealing of the helical fiber coil, final assembly of the sensor, and measurement of the Faraday response have yet to be conducted. To date, fiber annealing has only occurred for coils in a planar configuration [22]. Extending this technology to other coil shapes is still a challenge, bot likely one with a workable solution. In the annealing process, the singlemode optical fiber with its buffer coating is place in the annealing fixture. Pretreatment of the fiber by soaking in solvent, typically acetone or methylene chloride, allows the buffer to evaporate at a relatively low temperature in the annealing cycle (300 to $400{ }^{\circ} \mathrm{C}$ ), leaving the bare glass fiber to be annealed [22]. Ideally, a soft ceramic such as Macor ${ }^{\mathrm{TM}}$ is used to prevent scratching and fracturing of the fiber. The diamonite ceranic used in the travelling wave sensor design is much harder than Macor ${ }^{\mathrm{TM}}$, but it also has a signifficanly larger relative permittivity. One challenge is to determine if the fiber coil will survive the annealing cycle. A second challenge is to apply a protective coating on the fiber while it remains in the cerarnic fixture. Backfilling with a thinned polyurethane or silicone elastomer should provide a soft coat for the fiber.

In the absence of finished hardware and measured responses, the utility of the ceramic-filled travelling wave current sensor can only be evaluated from cell electrornagaetic characterization and modeled Faraday responses. Based on cell geometries and the relative permittivity of the ceramic dielectric, the higher order mode cutoff frequency for the transmission line is $3.6 \mathrm{GHz}$. The impedance of the cell was 
(a)

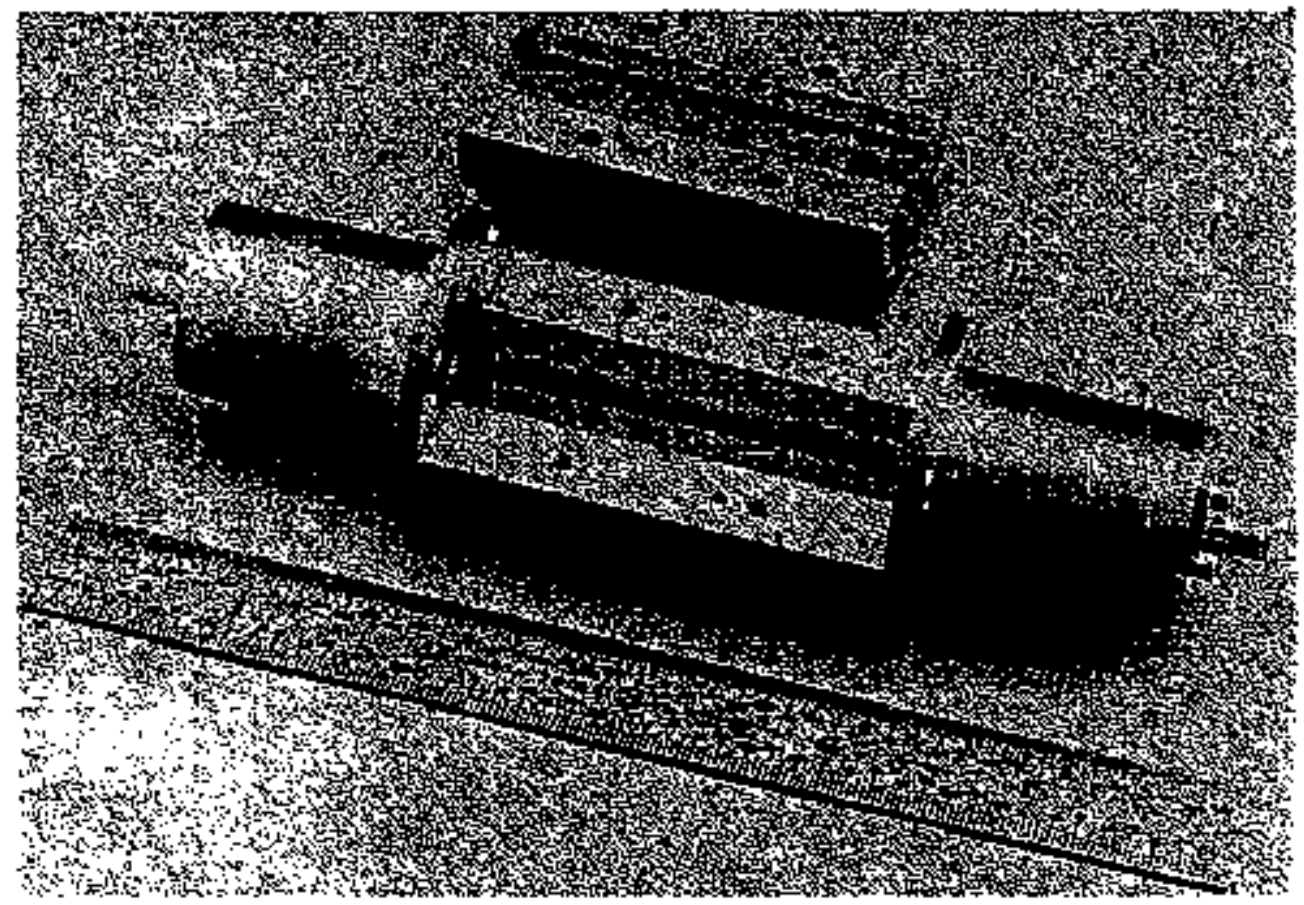

(b)

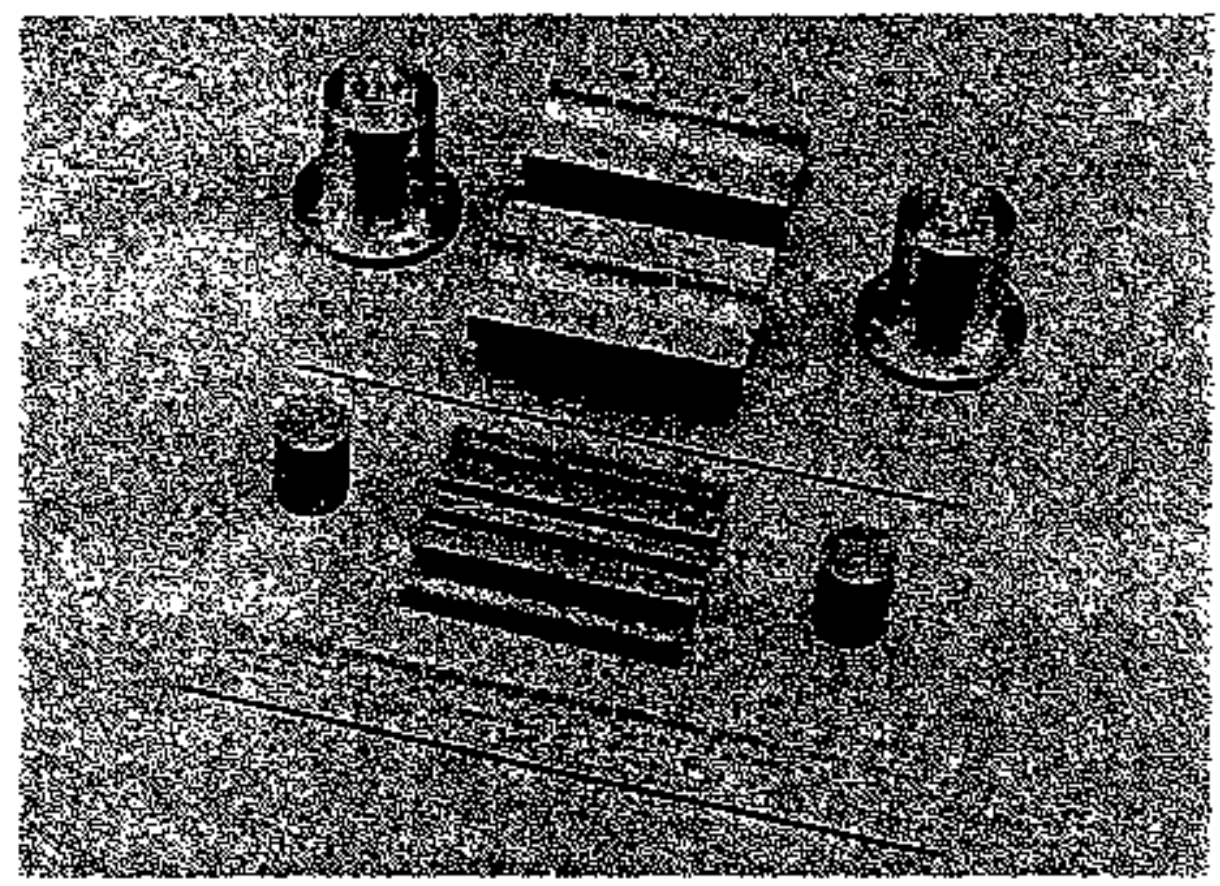

Fig. 12-2. Photographs of the ceramic dielectric travelling wave transmission cell. (a) A cutaway view of the cell showing the fiber mandrel guide: a 10-turn helical groove in the outer surface of the 6-mm ceramic tube. (b) The fully disassembled cell. 
characterized for frequencies below $5 \mathrm{GHz}$ A plot of the impedance magnitude is presented in Fig. 12-3. Oscillations in the impedance are created by reflections at the transition points into the cell dielectric volume. These cause the cell impedance to vary between $\sim 20 \Omega$ and $\sim 120 \Omega$ for the frequencies below $5 \mathrm{GHz}$. If the frequency range is limited to $2.5 \mathrm{GHz}$, a likely cutoff in the Faraday response characterization, impedance deviations about $50 \Omega$ are bound within $1.3 \mathrm{~dB}$. Also, below $2.5 \mathrm{GHz}$ the test cell has a very impressive SWR of 1.4.

The predicted Faraday response for this travelling wave sensor is shown in Fig. 12-4. Plotted are model-generated curves for both the magnitude and the phase. The

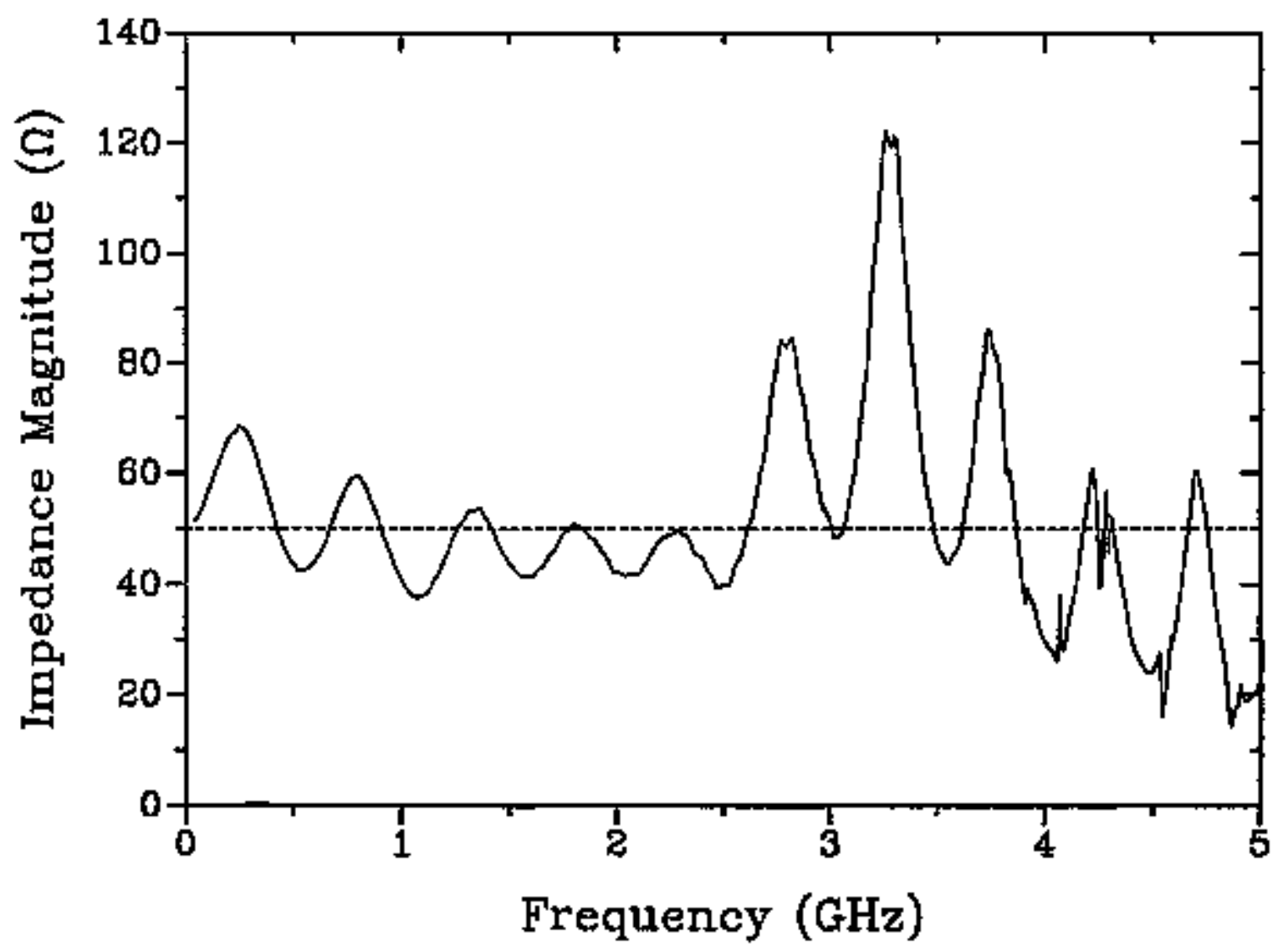

Fig. 12-3. The measured impedance magnitude of the ceramic dielectric transmission cell for frequencies below $5 \mathrm{GHz}$. The cell HOM cutoff frequency is $3.6 \mathrm{GHz}$. 

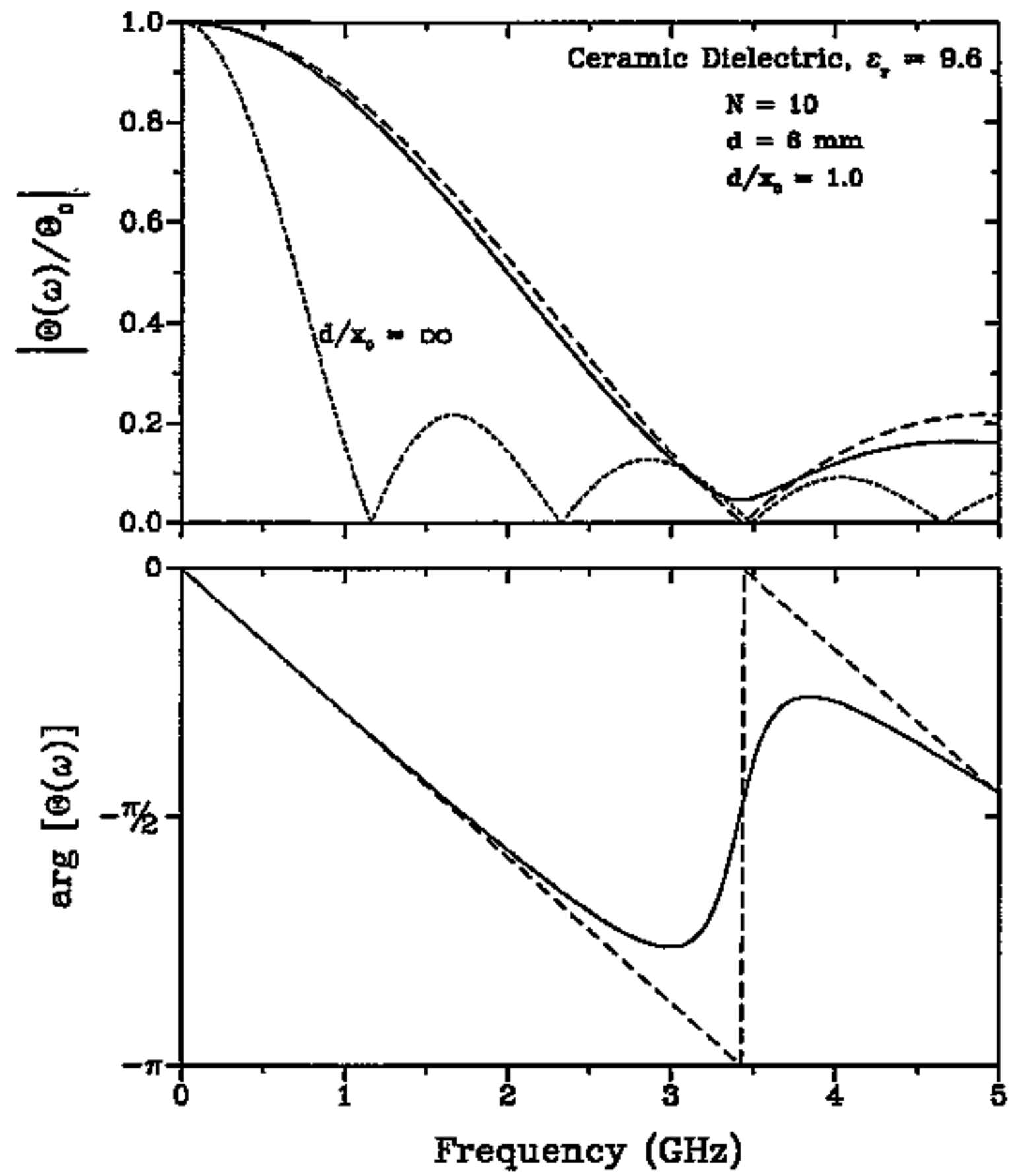

Fig. 12-4. The predicted frequency dependent magnitude (top) and phase (bottom) of the Faraday rotation for a travelling wave curent sensor using a ceramic dielectric and an annealed helictl fiber coil. The solid curve includes some dielectric dispersion; the dashed curve is for a system with no dielectric dispersion. 
solid curves are detemined using a model that includes a small amount of dielectric loss in the ceramic. These losses are based on a Debye molecular reorientation model much like the liquid dispersion treated earlier [7I]. Typical effects in the Faraday response result from the dielectric dispersion: some loss of bandwidth in the main response lobe, the missing null in the response magnitude, and the lack of maximum phase deviation at the frequency where zero magnitude usually occurs. The fiber coil leads have essentially no affect on the travelling wave sensor response because of their designed contiguration. Leads enter and exit the coil from the same direction making the transition to the helical shape over a minimal distance and with a radius of approximately one-half that of the coil. It is intendeo that the fiber annealing process will adeguately remove any linear birefringence in this lead transition region.

The projected sensor bandwidth is approximately $1.45 \mathrm{GHz}$, with a corresponding bandwidth enhancement factor of 2.8. It appears from Fig. 12-4 that response $\mathrm{S} / \mathrm{N}$ is large enough at $2.5 \mathrm{GHz}$ that measurements are possible up to this frequency (over the frequency range where the cell has a very good SWR). Estimated value for the sensitivity-bandwidth prodıct for this travelling wave device is $3.85 \mathrm{MHz}-\% / \mathrm{A}$, more than three times larger than that of the water-filled travelling wave current sensor. However, this SBP remains a factor of two below that measured for the bulk optic Faraday effect current sensor [30].

\section{Combination Fiber Sensors}

It is possible to use the helical fiber coil concept with the noncentered or noncircular, planar fiber coil concept to form a new travelling wave fiber current sensor configuration. The combination allows all of the separately-observed bandwidth and high frequency enhancements for these coil configurations to be utilized in a single sensor. The frequency dependent Faraday response for this combination is found directly from Eg. (3-7). In Chapter 3, two simplifying routes were developed, which led to the 
travelling wave coil configurations or the noncentered/noncircular coil configurations. Here no simplifications are made. To illustrate the combination sensor approach, a mathematical model is briefly outlined for a travelling wave fiber coil with a displaced (noncentered) conductor within the coil crass section.

The function $[(1 / r) \cos (\gamma)]$ in Eq. (3-7) describes the deviation in the Faraday interaction due to the nonconcentric axes of the current-carrying conductor and the fiber coil. Equation (6-3) for this function is valid for the combination sensor, but with $\mathbf{R}$ now Iepresenting the mean radial distance from the helical coil axis to any perpendicular point on the fiber, and the time dependence of the angle $\alpha$ now given by

$$
\alpha(t)=\frac{c}{n R \Delta L} t
$$

where $\Delta \mathbf{L}$ is defined by Eg. (8-11). Also, the reference angle in Eq. (6-3) is now expressed by $\alpha_{0}=\mathrm{ct} / \mathrm{t} /(\mathrm{n} R \Delta \mathrm{L})$. The phase factor in Eq. (3-7) is modified according to the developments in Chapter 8, leading to the form given in Eq. (8-4). Then an integral expression for the noncentered, travelling wave fiber corrent sensor is

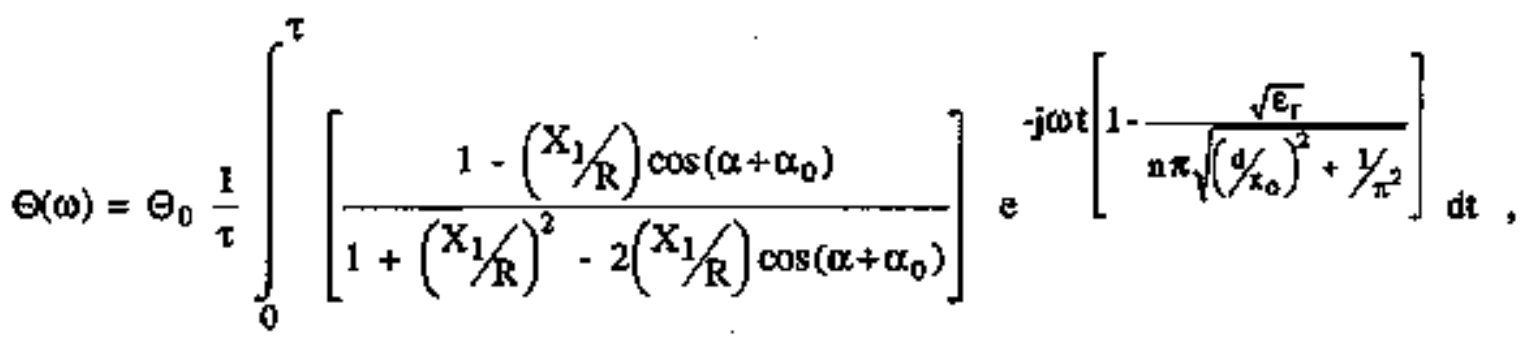

where $\tau$ is now the total transit time of light propagation throngh the helical fiber coil given by

$$
\tau=2 \pi \mathrm{RN}\left(\frac{\mathrm{n}}{\mathrm{c}}\right) \Delta \mathrm{L} .
$$


The solution to Eg. (12-2) proceeds as followed in Chapter 6 using long division decomposition of the first bracketed factor in the integral. The resulting series is integrated term by term and then simplifted to yield as expression similar to Eg. (6-14). The frequency dependent Faraday response for the combination sensor is

$$
\Theta(\omega)=\theta_{0}\left[\frac{\sin (\zeta)}{\zeta}\right] e^{-\mathrm{j} \zeta} \sum_{\mathrm{m}=0}^{\infty}(-1)^{2 m \xi}\left(\frac{\mathrm{X}_{1}}{\mathrm{R}}\right)^{\mathrm{m}}\left[1-\left(\frac{\mathrm{mN \pi}}{\zeta}\right)^{2}\right]^{-1}
$$

where $\zeta$ is given in Eq. (8-5b) for the travelling wave configurations and $\xi$ is the fraction of the single loop propagation delay taken from Eq. $(6-11)$. Both of these variables are functions of $\tau$ as given by Eq. (12-3).

The Faraday response predicted by Eq. (12-4) is simply that determined by Eq. (6-14) for a noncentered, planar coil expanded in the freguency domain to account for the travelling wave interaction. Figure 12-5 shows the predicted response for a two-turn, travelling wave sensor coil with $d=3 \mathrm{~cm}, d / x_{0}=1.0$, and $\xi=0.5$ and with the conductor fully displaced in the coil cross section, $X_{1}=R$. The fiber coil is treated as leadless. The displaced conductor creates a response resonance where initially the second null occurs in the centered-coil travelling wave response. This response is identical to the resonant behavior observed for the noncentered, planar coil predictions. The traveling wave characteristics of the sensor coil produce a large bandwidth enhancement (compare the dotted and dashed curves in Fig. 12-5.), and a smaller, but notable, enhanoement is created by the nonconcentric axes (dashed and solid curves). From Table 6-1, the BEF of the solid curve should be $5.8 \%$ larger. And as discussed in Chapter 6, the small enhancement due to the noncentered coil decreases as the number of fiber turns in the coil increases.

It is obvious from Fig. 12-5 that combining coil manipulation schemes with the travelling wave concept can enhance the high frequency response of the fiber cutrent 


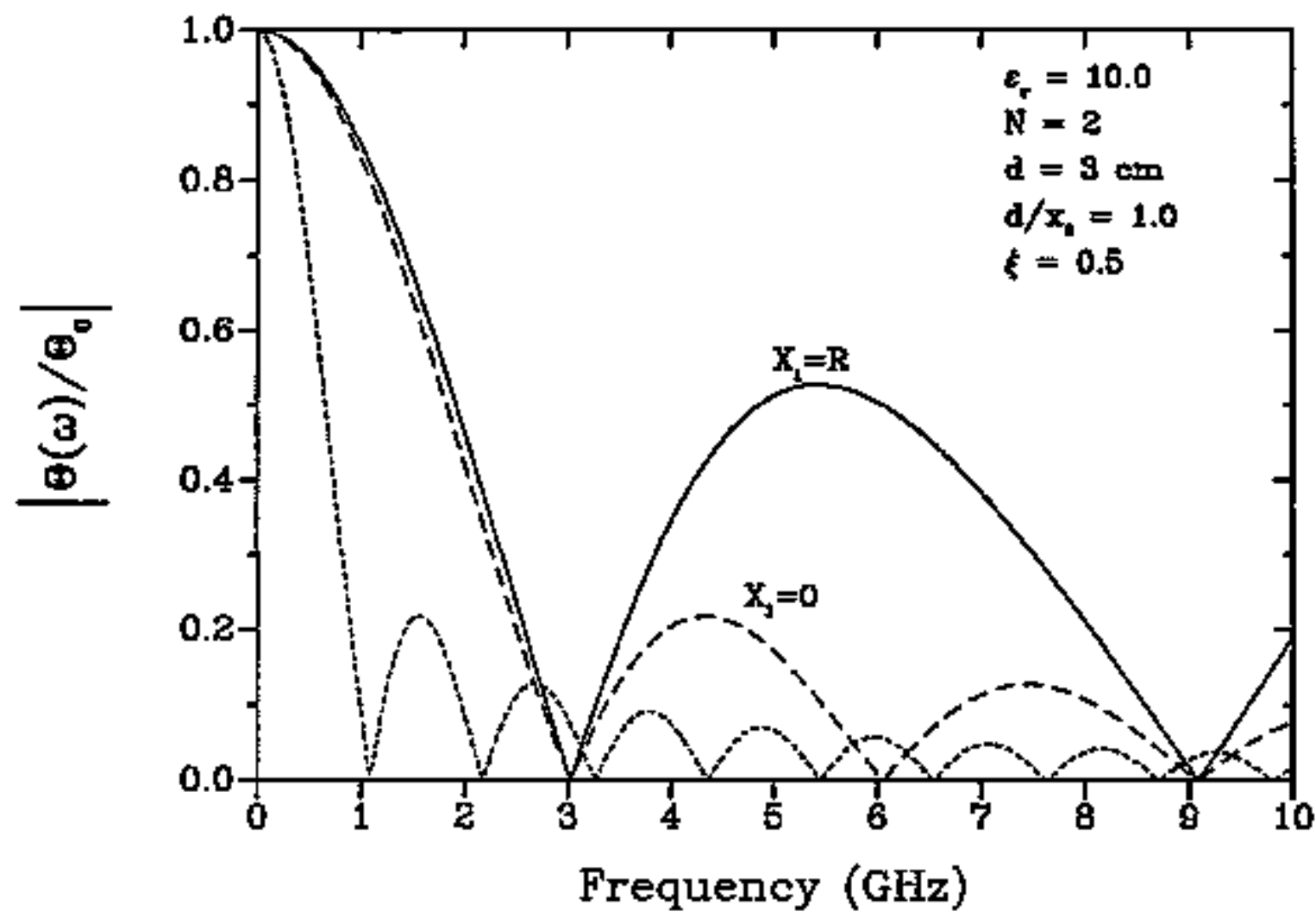

Fig. 12-5. The predicted relative magnitude of the Faraday rotation for a two-tum travelling wave fiber sensor with the current conductor fully displaced in the coil cross section. The dotted curve is the reference response for a centered, planar coil.

sensors. Other combinations of sensing concepts are certainly possible, and mathematical expressions to predict their Faraday response can be deveioped using the existing theories.

\section{Non-Fiber Configurations}

Several travelling wave corrent sensor configurations that utilize bulk optics or planar waveguides have been formulated. Three of these are discossed here to show options to the helical fiber travelling wave design. Some quantitative information is provided, but most of the discussion is conceptual. 


\section{Helical Waveguide Sensor}

The ceramic dielectric traveling wave current sensor, with an annealed helical coil, offers significant reduction in size and Jarge improvement in the sensitivitybandwidth product compared to the liquid travelling wave configuration, but absolute Faraciay sensitivity still suffers. The only mechanism for improving the static response is to add fiber turns. However, this will increase the length of the transmission cell as further reductions in coil diameter (even using the annealing process) do not appear feasible. A planar waveguide structure fabricated on the surface of a cylindrical substrate could provide the optical propagation medium. Envisioned is a singtemode rib waveguide etched on the surface of a glass or crystal which has a high Verdet constant. Singlemode waveguides are typically a few micrometers in dimension, and such structures have not been demonstrated on other than flat surfaces. A real challenge exists in fabricating such a waveguide, especially in a helical layout. Significant reductions in waveguide belix diameter are possible with this concept.

The terbium-doped borosilicate glass, FR-5, has a Verdet constant that is approximately 20 times larger than that of a silica fiber (see Table 2-1) $[39,40]$. A waveguide fabricated from this material would significantly improve the Faraday sensitivity of a current sensor. FR-5 glass has a relatively high loss per unit length for a waveguide medium [25], but the total attenuation shoutd be small since the proposed light transit distance is short. If a travelling wave light path is created with a mean diameter of $2 \mathrm{~mm}, 10 \mathrm{turns}$ and $\mathrm{d} / \mathrm{x}_{0}=1.0$, the sensor bandwidth is predicted to be $-2 \mathrm{GHz}$. The projected sensitivity-bandwidth product is approximately $106 \mathrm{MHz}-\% \mathrm{~A}$. These computations assume a laser wavelength of $633 \mathrm{~nm}$, so that $\mathrm{n}=1.684$ for the FR-5 glass [40], and that the glass medium is the sole dielectric in the coaxial line. An estinate of the relative pernittivity of the FR.5 is 5.0 in the $1 \mathrm{MHz}$ to $1 \mathrm{GHz}$ frequency range based on handbook values for similar glasses [76]. If orly a thin cylinder of the FR-5 sobstrate is used along with a higher $\varepsilon_{\mathrm{r}}$ ceramic as the remaining dielectric, the 
bandwidth can be enhanced even further. Assuming $\varepsilon_{\text {eff }}=9.0$ for the slow-wave structure, the sensor will have a $\Delta B \approx 2.6 \mathrm{GHz}$ and a $\mathrm{SBP}=137.5 \mathrm{MHz}-\%$. The waveguide will only be $2 \mathrm{~cm}$ long, and the entire device package should not exceed one inch long by half inct in diameter, excluoing the RF connectors and the supporting optoelectronics needed for light production and detection.

The challenge of mating miniature lenses, polarizers and optical fiber to the waveguide structure is not addressed here, however, these technologies are becoming quite advanced and should not be a liniting factor. If the complete sensing system can be fabricated and assembled, it wonld provide milliampere current detection at greater than $100 \mathrm{MHz}$ bandwidth. These figures rival or supass conventional elecirical current monitors [4], with the added advantages afforded by the noninterfering optical technig̨ues.

\section{Bulk Ontic Sensor}

Another prospective travelling wave current sensing technique utitizes a bulk optic Faraday rotator material and reverses the optical and current-carrying paths in the structuke. The current passes through a wire solenoid wound around a cylinder of the optical material. Figure 12-6 shows a schematic drawing of the sensor. The magnetic field inside the solenoid is axial and scales linearly with both the current and the number of wire turns. The light is injected along the cylindrical axis of the Faraday material and is codirectional with the magnetic field. Sensitivity of this device can be controlled by varying the wire tums and the choice of optical materials. In this scheme the propagating light will outpace the magnetic field since it travels a shorter path. Bandwidth will depend on phasing between these two signals.

This technique should produce small, yet responsive, current monitors when implemented. As an example, FR-5 glass is selected as the Faraday rotator material and cast in a cylinder $2 \mathrm{~mm}$ in diameter and $2 \mathrm{~cm}$ long. Forty toms of wire (30 or 32 gavge 


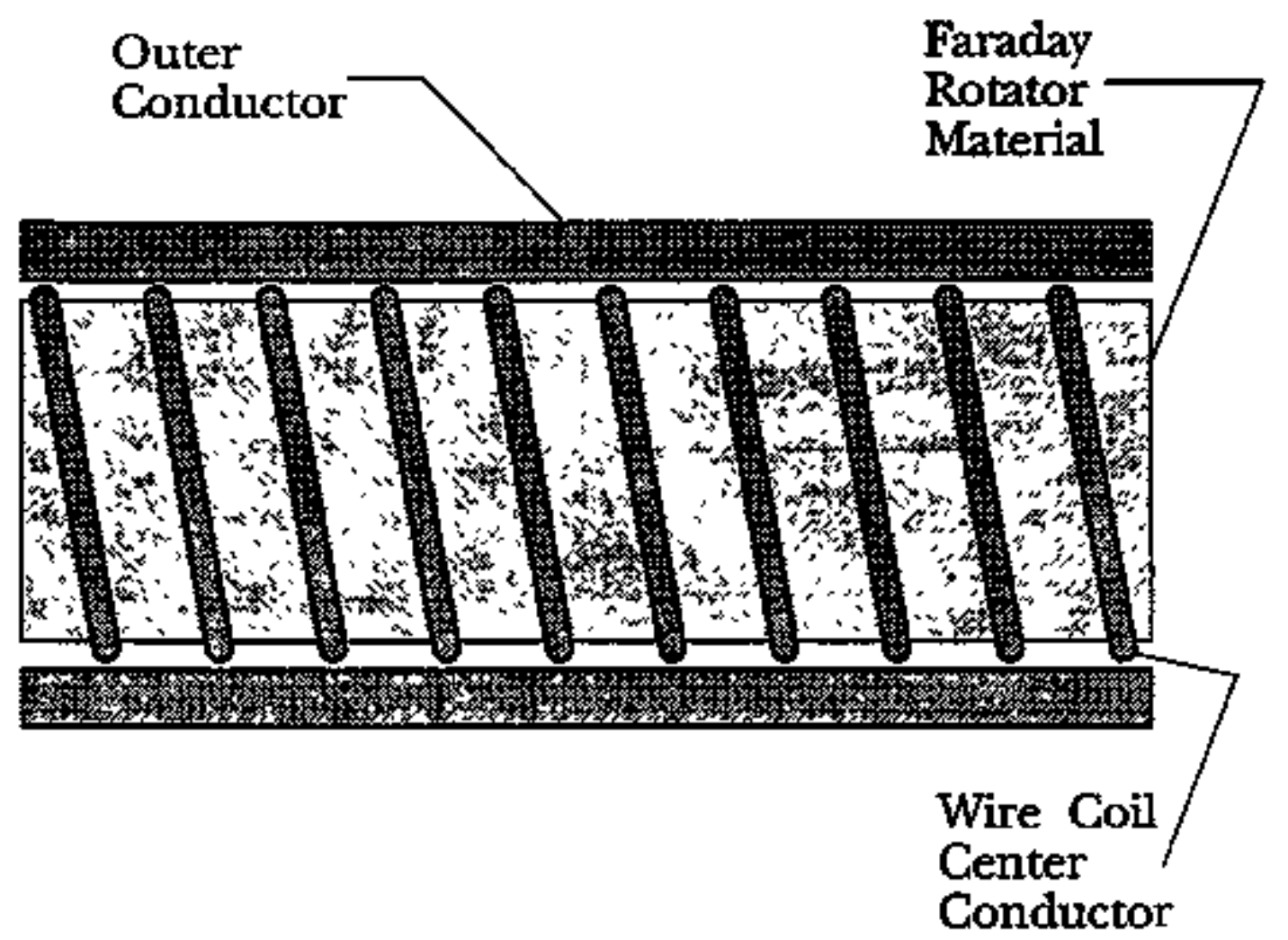

Fig. 12-6. A wideband bulk optic cursent sensor with a helical wire coil used to generate an axial magnetic field inside a cylindrical piece of Faraday rotator material.

should be small enough, yet capable of handling sufficient current) is wrapped in a helical solenoid around the glass. Because of the helix pitch, $0.5 \mathrm{~mm}$, the magnetic field phase velocity along the solenoid axis is approximately an order of magnitude smaller than that of the propagating light. Consequently, the system $3 \mathrm{~dB}$ bandwidth should be limited to approximately $250 \mathrm{MHz}$. The measurement sensitivity of this device, however, is quite high, with an estimated noise equivalent corrent at foll bandwidth of $0.75 \mathrm{~A}$ and a grojected sensitivity-bandwidth product of approximately $51 \mathrm{MHz}-\%$.

One design difficulty with the wire solenoid is its large reactance at the higher frequencies. Near the sensor $3 \mathrm{~dB}$ bandwidth, the inductive reactance exceeds $500 \Omega$ which can limit signal current propagation. By adding distributed capacitance through 
use of a transmission line ground plane, this effect is countered [77]. The coaxial line structure shown in Fig. 12-6 is such an implementation. The device characteristic inpedance can be returned to $50 \Omega$ by maintaining a spacing of a few mils ( $0.2 \mathrm{~mm}$ ) between the solenoid and the outer coaxial conductor. By choosing a thin electrical insulating material soch as mytar for the spacer, the relative permittivity is closely matched with that of the FR-5 glass, and the electromagnetic properties of the transmission line are optimized.

\section{Planar Waveguide Sensor}

A third travelting wave sensor configuration incorporates a serpentine singlemode planar waveguide in the dielectric substrate of an RF stripline. This structure is shown in the schematic of Fig. 12-7. The waveguide is perpendicular as it passes under the RF trace, and the propagating light interacts $\mathrm{N}$ times with the magnetic field. Each interaction is over a very short distance so the Faraday response behaves moch like that for the delta function interaction described in Chapter 7. Only with this current sensor, the sign of B $+d$ changes with each consecutive pass by the conductor. A combination of previously developed techniques and models can be userd to predict responses.

The frequency dependent Faraday rotation is determined by introducing the travelling wave phase factor, $\exp \left[-j \omega t\left(1-v_{\text {ligh }} / v_{\mathrm{ph}}\right)\right]$ into $\mathrm{Eg}$. (7-4) and accounting for the counter-rotating interactions asing $(-1)^{\mathrm{m}}$ in the series. The resulting delta function response has the form

$$
\Theta_{8}(\omega)=\theta_{0} \frac{1}{N} \sum_{m=0}^{N-1}(-1)^{m} e^{-j m \frac{\omega \tau_{1}}{2}(1-\zeta)},
$$

with

$$
\tau_{1}=\frac{n}{c} L_{1},
$$



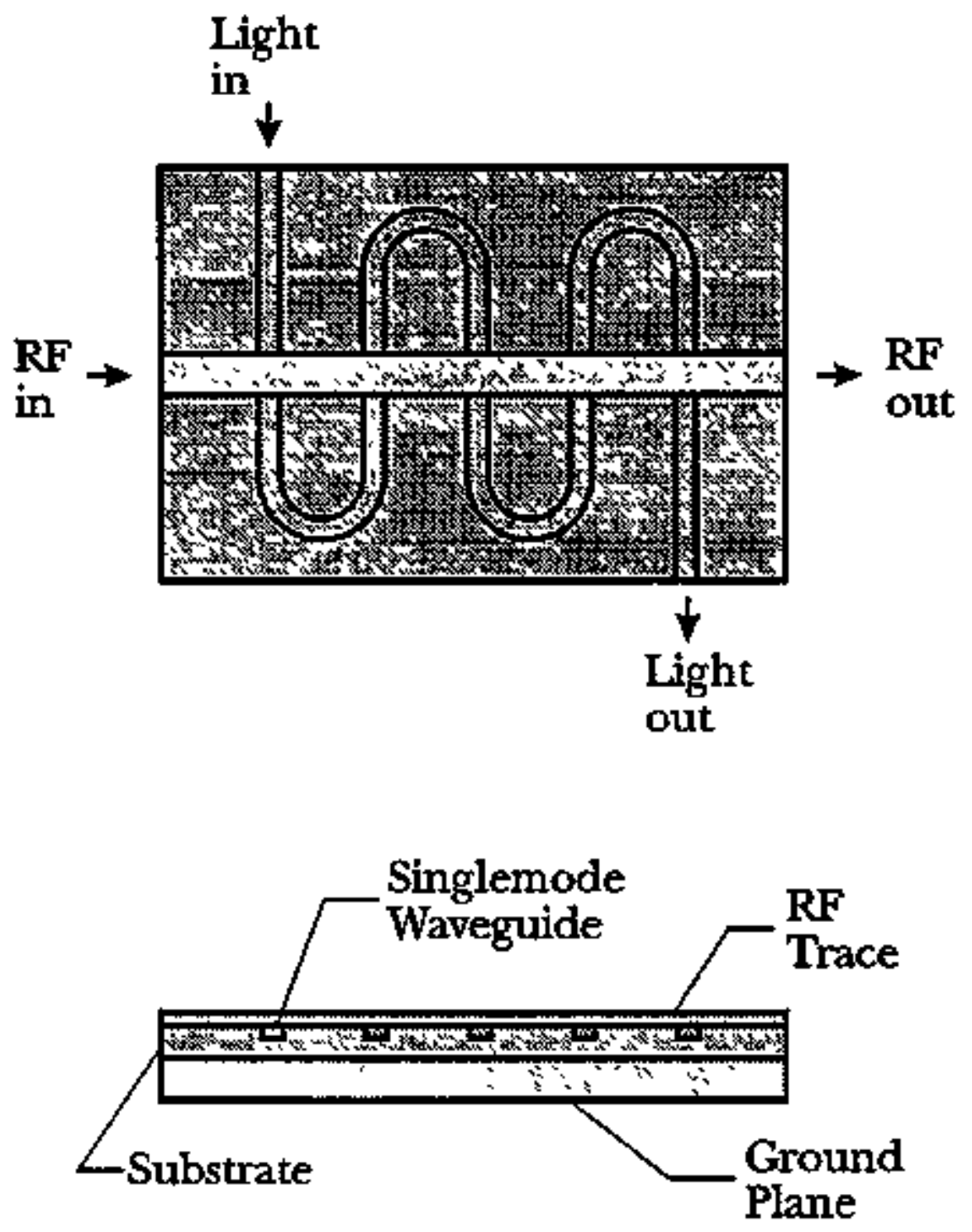

CROSS SECTION

Fig. 12-7. A plarar waveguide travelling wave optical current sensor. The waveguide follows a serpentine path that makes several passes by the stripline conductor. 
and

$$
\zeta^{\prime}=\frac{\sqrt{\varepsilon_{\mathrm{r}}}}{\mathrm{n}} \frac{\mathrm{x}_{0}}{\mathrm{~L}_{1}} \text {, }
$$

where $x_{0 / 2}$ is the distance along the siripline between waveguide interactions, and $L_{1} / 2$ is the light propagation distance between interactions. In Eq. (12-5) any arbitrary phase delays between the light and the magnetic field have been ignored.

The response predicted by Eq. (12-5) is onique in that it depends on $\mathrm{N}$ being even or odd and the degtee of travelling wave phase matching. At the low freguencies, $\omega \rightarrow$ 0 , the relative response is

$$
\frac{\Theta_{\delta}(0)}{\Theta_{0}}=\frac{1}{N} \sum_{m=0}^{N-1}(-1)^{m}=\left\{\begin{array}{cl}
0 & \text { if } N \text { is even } \\
\frac{1}{N} & \text { if } N \text { is odd }
\end{array} .\right.
$$

The maximum relative response, $\Theta_{0}$, is not attained at the low frequencies (unless $\mathrm{N}=1$ ) since every second pass of the light by the conductor negates the response of the previous pass. Maximum response will involve alt $\mathrm{N}$ interactions and is given by

$$
\Theta_{0}=\mathrm{N} \vee \mathrm{B} \ell
$$

where $\ell$ is the short interaction length between the light and the field as the waveguide passes near the RF concuctor.

Equation (12-8) also describes the Faraday response for perfect phase ratching between the two propagating signals. When the travelling wave condition is met, $\varsigma^{\prime}=1$, and the phase factor in Eq. (12-5) is again equal to one. Thus, under ideal travelling wave conditions and with $\mathrm{N}$ odd, the response magnitude is $1 / \mathrm{N}$ for all frequencies. This 
implies that optimum sensors with near perfect phase matching only require that $\mathrm{N}=1$.

For less than perfect phase matching, the sensor Faraday response exhibits resonances, as seen previously for the delta function interactions. In Fig. 12-8 the response relative magnitude is plotted versus the normalized frequency, $\omega \tau_{1 / 2}$, for a waveguide device making three passes by the conductor. The use of the nornalized frequency for the plot abscissa removes structure dimensions from the computations and presents the response in a form consistent with those in Chapter 7. The degree of phase matching is quite important in determining the freguency responses shown in Fig. 12-8.

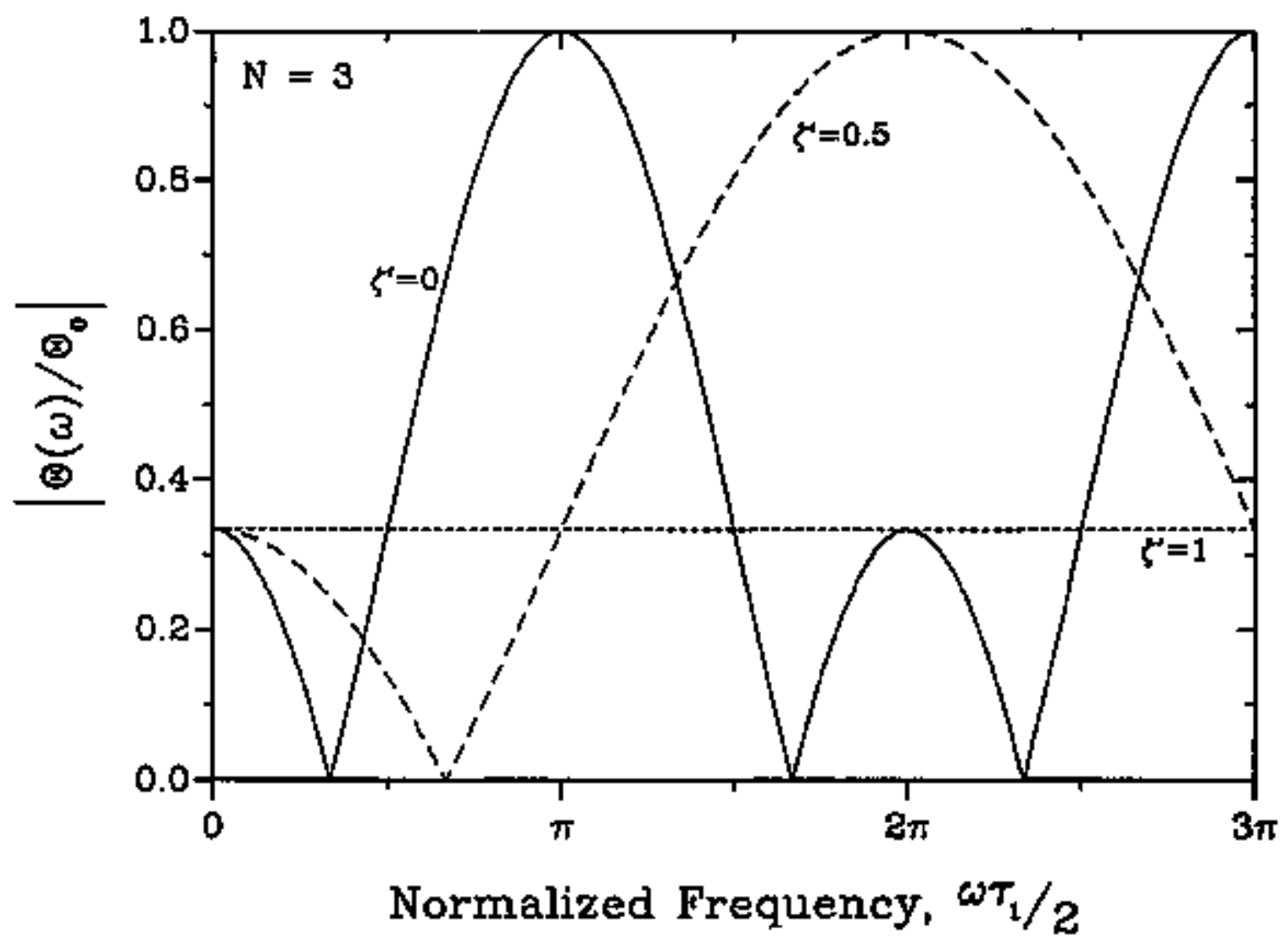

Fig. 12-8. The predicted relative magnitude of the Faraday rotation for the planar waveguide traveling wave current sensor. The waveguide makes three passes under the RF trace. Three phase matching conditions are shown: none $\left(\zeta^{\prime}=0\right)$, intermediate $\left(\varsigma^{*}=0.5\right)$ and perfect $\left(\varsigma^{\prime}=1\right)$. 
Resonance maxima occur when

$$
\frac{\omega \tau_{1}}{2}\left(1 \cdot \varsigma^{\prime}\right)=\left(2 m^{\prime}+1\right) \pi, \quad m^{\prime}=0,1,2, \ldots .
$$

The frequency dependence of the maxima on $N$ is removed since the transit time $\tau_{1}$ is used. But the number of nells occurring between the response peaks is $\mathrm{N}-1$. For frequencies below the first maxima, there are $1 / 2(\mathrm{~N}-1)$ nulls if $\mathrm{N}$ is odd and $\mathrm{N} / 2$ nulls (including the one at zero frequency) if $\mathrm{N}$ is even. Also, the bandwidth of the high frequency resonances is determined by the total transit time $\tau=N\left(\tau_{1 / 2}\right)$. If the number of waveguide passes under the stripline gets quite large, $N \rightarrow \infty$, the response becomes concentrated at frequencies near resonance, approaching zero relative magnifude between the maxima.

One of the advantages of this planar waveguide current sensor is its large narrowband, high frequency response. By tailoring the device parameters, these high frequency bands can be utilized. (As discussed in Chapter 7, the resonant response does not continae to infinity bat is bound by an enveloping function that depends only on the interaction length $\ell$.) As an example, the characteristics of a practical waveguide sensor are estimated. The example device uses a substrate material $0.5 \mathrm{~mm}$ thick with a relative permittivity of 5.0. The singlemode waveguide has a refractive index of 1.68 (FR-5 glass) and makes five passes by the conductor, every $1 \mathrm{~cm}$ along the light path and every $2.5 \mathrm{~mm}$ along the current path $\left(\mathrm{x}_{0} / \mathrm{L}_{1}=0.25\right)$. The $50 \Omega$ stripline has a trace width of $\sim 1.7 \mathrm{~mm}$. An estimate of the magnetic flex density along the light path at each interaction point is $B \approx 0.0025 \mathrm{~T} / \mathrm{A}$, which produces a peak Faraday rotation of $\Theta_{0}=$ $0.087 \%$ A. The static rotation is $1 / 5$ this value or $0.017 \%$. Fregrency parameters are quite impressive: the first null in the response curve occurs at $2.68 \mathrm{GHz}$, the first resonance maximum with relative magnitude of one occurs at $13.4 \mathrm{GHz}$, and the 
resonance response bandwidth is $2.37 \mathrm{GHz}$. The bandwidth of the lowest frequency response lobe is approximately $1.2 \mathrm{GHz}$. As a comparative measure, the SBP for this device is only $20.4 \mathrm{MHz}-\% / \mathrm{A}$ at low freguencies, but has an SBP of $204 \mathrm{MHz}-\%$, or ten times larger, in the resonance frequency band. 


\section{CHAPTER 13}

\section{CONCLUSIONS}

A thorough investigation has ben made of the high frequency response of planar and travelling wave Faraday effect optical fiber current sensors. Several models have been developed that provide excellent response predictions for fiber coils in a variety of sensor configarations. Measurements of the magnitude of the high frequency response for the sensors verify many of the model predictions. Thus, some unique Faraday effect current sensors have been dernonstrated and reported for the first time as a result of this study.

Three basic configurations of planar coil fiber current sensors were explored: circuiar cross section coils with coincident coil and conductor axes; circular coils with the conductor displaced from the coil axis; and noncircular and highly elongated coils with the conductor centered or fully displaced in the coil cross section. Travelling wave current sensors were also explored. They consisted of helical fiber coils with constant diameter immersed in the dielectric of a coaxial transmission line. Both air and liguid dielectrics were used in these transmission lines. Both groups of current sensors, pianar and travelling wave, required the development of coaxial transmission test cells [34] to properly characterize the fiber coils at high frequencies (up to $1 \mathrm{GHz}$ ).

Planar fiber current sensors with a circular coil cross section and concentric coil/conductor axes have an easily predictable high frequency resposse determined by light transit time in the fiber. Many measurements were made with coils of varying radii and nambers of turns to demonstrate this concept. All tests extended in frequency beyond the cutoff frequency of the sensors and, in some cases, covered several of the higher frequency response lobes. Of particular interest in this sensor category were measurements using a $20 \mathrm{turn}, 6.4 \mathrm{~mm}$ diameter coil which had been annealed to remove bend-induced linear birefringence [21,22]. This sensor has a bandwidth of $229 \mathrm{MHz}$ and 
a sensitivity-bandwidth product estimated at $0.8 \mathrm{MHz}-\%$. The measurements on this sensor verified that the annealing process can successfully be used to manufacture small radii coils with improved sensitivity-bandwidth products and without the detrimental birefringence effects.

Important in the correct modeling of the frequency dependence of fiber current sensors are the effects of leads. They add to the total length of frber that the light must traverse and can result in reduced detection bandwidths. In some configurations, the fiber leads add an effective half turn to the coil length, which significantly alters high frequency response, especially for coils with small numbers of tarns.

Noncentered and noncircular planar coils used as frber sensors exhibit resonances in their high frequency response. Resonance magnitudes are dependent on the displacement distance between the coil and conductor axes and the eccentricity of the coil cross section. Spacing of the resonance frequencies depends on the length of fiber in a single coil turn. This type of response at the higher frequencies is compared to similar respotses that occur in tapped and recirculating fiber delay line devices [64,65]. To aid in the characterization of the high freguency Faraday rotation for a coil with a highily elongated cross section and the conductor at maximum displacement, a delta function model was introduced. This model is based on a point interaction between the magnetic field and the light, and it provides an excellent theoretical framework for building frequency response functions for sensors that exhibit bounded resonant behavior. A rectangular cross section coil model was used to predict the frequency response for sensors with arbitrary coil cross section and with arbitrary placement of the conductor within the coil. A connection between the noncentered, circular coil and the highly elongated coil was provided by the rectangular coil model as it allowed for varying eccentricity of the cross section. One additionally interesting feature is that resonant responses at high frequencies occur for fiber current sensors with the conductor outside the coil cross section, even when such response is prohibited at the low frequencies as 
described by Ampere's law.

Single-turn and few-turn planar fiber coils exhibit sensor $3 \mathrm{~dB}$ bandwidths that are significantly affected by coil configuration. The maximum bandwidth for these sensors is realized for a highly elongated, one-turn coil (a delta function interaction); ideally the bandwidth approaches infinity. Light entry point into the single-turn, circular coil can create a factor of two difference in response bandwidth when the conductor is fully displaced in the coil cross section. If light enters away from the radial condactor offset, a large ( 50\%) bandwidth increase is observed compared to the centered coil; if light enters nearest the conductor, a significant lass $(-29 \%)$ in bandwidth occurs. Bandwidth increases in single or few-turn coils are farther enhanced by increasing the eccentricity of the coil crass section. Using the delta function response model, a two-turn coil will exhibit a $13 \%$ increase in bandwidth. These bandwidth deviations become less significant as the number of coil turns increases, regardless of the coil configuration. For multiturn coils with $\mathrm{N} \geq 10$, all fiber sensor bandwidths converge to that of the simple circular, centered coil, Eg. (5-7). This simplifies carrent sensor coil design, especialty for those applications where the frequency range of interest lies below the main lobe $3 \mathrm{~dB}$ bandwidth.

The travelling wave configurations show that increases in bandwidth are possible for fiber current sensors without a loss in detection sensitivity. By distributing the fiber coil and slowing the propagation of the magnetic wave, improved phase malching occurs. Bandwidth enhancement is a function of the helical coil diameter-to-pitch ratio and the relative pernittivity of the transmission line dielectric. Under ideal conditions, a configuration exists where, theoretically, a near-infinite bandwidth could occur. Realistically, such a large bandwidth is not possible, but enhancement factors of approximately 10 (compared to an equivalent planar coil) appear achievable.

Transmission test cells filled with liguid provided the slow-wave structures for travelling wave sensor experimental measurements. Liquids were chosen as coaxiå line 
dielectrics because they have large relative permittivities and can easily fill the volume of the cell around the fiber sensor. However, liquids also exhibit significant dielectric dispersion at the higher frequencies [71] and require other structures to support the fiber coil within the cell. These properties can severely limit the system bandwidth. Extended mathematical models of the travelling wave curent sensor have been developed that account for many of the properties of the transmission cell structures [75]. In addition to liquid dispersion, the models include waveguife dispersion of the lowest-order TM mode in the transunission line, an effective relative permittivity doe to the teflon support structures, and effects from excess lead length used in a non-travelling configuration. The models only consider a single EM mode in the coaxial line and assume that mode has quasi-TEM characteristics. In most cases the extended models showed excellent agreement with the experimental results.

Three liguids - 1-propanol, methanol and water - were used in the test cells. Their progressively larger relative permittivities $-20.1,32.6$, and 78.5 , respectively at $25^{\circ} \mathrm{C}$ - created sensor systems with increasingly larger bandwidth for a given helical coil structure. Comparative tests with a helical coil in a coaxial airline indicated that merely distributing the coil provides minimal bandwidth enhancement (maximum of 1.37); slow-wave mechanisms are needed for further phase matching. The largest bandwidth recorded during experimentation was approximately $300 \mathrm{MHz}$ for a waterfilled cell and a 14-tum helical fiber coil with $3 \mathrm{~cm}$ diameter and $3 \mathrm{~cm}$ pitch. This sensor has a bandwidh enhancement factor of $\sim 4.1$ when compared to a 14-turn, $3 \mathrm{~cm}$ planar coil, Its estimated sensitivity-bandwidth product is $1.06 \mathrm{MHz}-\% / \mathrm{A}$, the largest SBP measured to date for a fiber current sensor.

Models of the travelling wave fiber sensor configurations predict that high frequency response will be limited in liquid-filled devices. Large bandwidth enhancements or higher frequency operation will require soltd dielectric slow-wave stmctures in combination with annealed fiber coils or helical waveguides. Predictions 
for such systems indicate that bandwidths greater than $2 \mathrm{GHz}$ and SBPs greater than 100 MHz- $\%$ A are possible. In addition to enhanced performance, these devices will also significantly reduce sensor size. Other non-fiber travelling wave sensor configurations may ultimately prove to be more sensitive in given current monitoring applications. A proposed planar waveguide travelling wave sensor fabricated in an RF stripline shows excellent response potential. With proper selection of Faraday rotator materials for the waveguide and with optimum serpentine path design, enhanced high frequency operation is predicted well into the microwave region with SBPs as large as $200 \mathrm{MHz}-\%$.

In summary, this work has explored the theoretical and experimental high freguency characteristics of Faraday effect fiber current sensors. Detailed quantitative analyses of the frequency responses of planar and travelling wave sensor configurations are now possible. High frequency response functions depend on several properties of the fiber coil and the transmission medium surrounding the coil. Sensor bandwidth can be enhanced through careful travelling wave fiber design or through selected use of few-turn noncentered and elongated fiber coils. Narrowband, high frequency resonant enhancement is produced through proper design with the noncentered or noncircular fiber coils. Measurements have been performed at frequencies that extend into the microwave region with $1 \mathrm{GHz}$ being the maximum used. All of the experiments explore high fregaency regions (usually above the $3 \mathrm{~dB}$ bandwidth) not previously considered for sensitive, Faraday effect fiber current sensors. Many of the sensors show adequate $\mathrm{S} / \mathrm{N}$ and characterizable responses in these regions. Models of the sensor systems have allowed further investigations of potential response in these high frequency regions. Thns, it appears feasible to fabricate current sensors from optical fibers (or optical wavegraides) that have a wider bandwidth or higher frequency detection capability than conventional current probes. 


\section{APRENDDX A \\ EXPANDED COAXIAL TRANSMISSION CELLS}

Expanded coaxial transmission cells have been developed to provide a known electromagnetic environment for the testing of optical fiber corrent sensor coils [34]. These structures are the coaxial analog to the rectangular transverse electromagnetic (TEM) cells that are used extensively in RF testing [78,79]. Large working volumes are characteristic of the transmission cells, able to contain sizable test objects with minimal perturbation of the impinging TEM wave. The coaxial cell geometry is ideal for experimentation with optical fiber coils that measure conducted RF currents; the known modal properties and impedances make it easy to relate corrent to power at fregnencies well into the microwave region.

\section{Coaxial Line Properties}

The waveguide theory for an ideal coaxial cable is well devejoped $[73,80]$, but it is repeated in a brief format here to highlight some of the nseful properties and to provide a reference for later theoretical developments.

The fields inside the dielectric medium of a coaxial line (see Fig. A-1) with center conductor radius $\rho=\mathrm{a}$ and outer conductor radius $\rho=\mathrm{b}$ can be divided into transverse magnetic (TM) and transverse electric (TE) components. The TM components of the electric and magnetic field are given by

$$
\begin{gathered}
E_{z}=u^{2}\left[A J_{m}(u \rho)+B Y_{m}(u \rho)\right] \cos (m \phi), \\
H_{\phi}=\frac{j \omega \varepsilon}{\beta} E_{\rho}=-\frac{j k^{2}}{\mu \omega}\left[A \frac{\partial}{\partial \rho} J_{m}(\nu \rho)+B \frac{\partial}{\partial \rho} Y_{m}(u \rho)\right] \cos (m \phi),
\end{gathered}
$$

and 


$$
E_{\phi}=-\frac{j \omega \varepsilon}{\beta} H_{\rho}=\frac{m \beta}{\rho}\left[A J_{m}(u \rho)+B Y_{m}(u \rho)\right] \sin (m \phi) .
$$

The TE field components are given by

$$
\begin{gathered}
H_{z}=u^{2}\left[C J_{m}(u \rho)+D Y_{m}(u \rho)\right] \cos (m \phi), \\
E_{\phi}=-\frac{j \omega \mu}{\beta} H_{\rho}=j \mu \omega\left[C \frac{\partial}{\partial \rho} J_{m}(u \rho)+D \frac{\partial}{\partial \rho} Y_{m}(u \rho)\right] \cos (m \phi),
\end{gathered}
$$

and

$$
H_{\phi}=\frac{j \omega \mu}{\beta} E_{\rho}=\frac{m \beta}{\rho}\left[C J_{m}(u \rho)+D Y_{m}(u \rho)\right] \sin (m \phi) .
$$

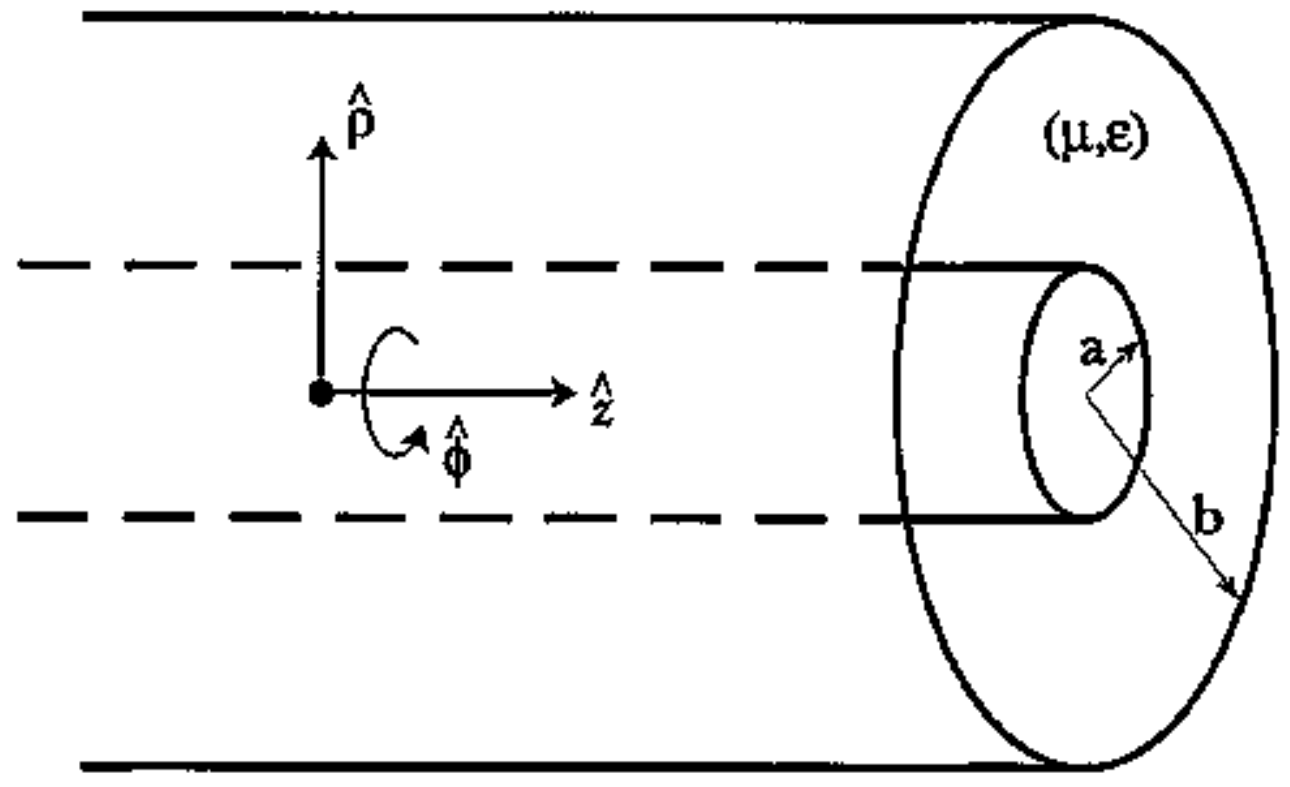

Fig. A.1. The geometry of a coaxial transmission line. A dielectric medium with permeability $\mu$ and permittivity $\varepsilon$ falls the volume between cylindrical conductors of radius $a$ and $b$. The cylindrical coordinates $(\rho, \phi, z)$ as indicated are used for computations. 
For brevity, the common phase factor $e^{-\beta z}+j \omega t$ is omitted from all the components in Eqs. (A-1) and (A-2). Here

$$
\mathrm{u}^{2}=\mathrm{k}^{2}+\beta^{2} \text {, }
$$

with

$$
\mathrm{k}^{2}=\mu \varepsilon \omega^{2},
$$

and $\beta$ the wave propagation constant. The coefficients A, B, C, and D are arbitrary constants, and $\mathrm{J}_{\mathrm{m}}$ (up) and $\mathrm{Y}_{\mathrm{m}}(\mathrm{u} \rho$ ) are Bessel functions of order $\mathrm{m}$ of the first and second kind, respectively. If the coax has infinitely conducting walls, the tangential components of the electric field vanish at both $\rho=a$ and $\rho=b$. This leads to two eigenvalue equations describing the TM and TE modes:

$$
\frac{Y_{m}(u a)}{J_{m}(u a)}=\frac{Y_{m}(u b)}{J_{m}(u b)},
$$

for the TM modes, and

$$
\frac{Y_{m}^{\prime}(u a)}{J_{m}^{\prime}(u a)}=\frac{Y_{m}^{\prime}(u b)}{J_{m}^{\prime}(u b)}
$$

for the TE modes. The primes in Eq. (A-6) denote differentiation with respect to $p$.

The lowest order mode in the ideal coaxial line is both TE and TM or TEM; this requires that $E_{2}=H_{2}=0$ for all $p$. Using Eqs. (A-Ia) and (A-2a), these conditions are satisfied only if $u^{2}=0$. Then $\beta=j k$, and the TEM wave propagates with a phase velocity given by

$$
v_{\mathrm{ph}}=\frac{\omega}{k}=\frac{1}{\sqrt{\mu \varepsilon}},
$$


which is the speed of light in the dielectric medium. The TEM mode is azimuthally symmetric ( $m=0$ ), and its two transverse field components can be derived from $\mathrm{Eg}$. (A. lb). They are given by

$$
H_{\phi}=\frac{E_{p}}{\eta}=\frac{i}{2 \pi \rho}
$$

where

$$
\eta=\sqrt{\frac{\mu}{\varepsilon}}
$$

is the wave impedance and $i$ is the conducted current. The characteristic impedance of the TEM mode is

$$
\mathrm{z}_{0}=\frac{\eta}{2 \pi} \ln (\mathrm{b} / \mathrm{a})
$$

For a dielectric medium containing no magnetic materials, $\mu=\mu_{0}$, and with a relative pernittivity, $\varepsilon_{r}=\varepsilon / \varepsilon_{0}$, Eg. $(A+10)$ becomes

$$
\mathrm{Z}_{0}=\frac{60}{\sqrt{\varepsilon_{\mathrm{r}}}} \ln (\mathrm{b} / \mathrm{a})
$$

Equations (A-5) and (A-6) provide for an infinite number of real solutions for $u$ for a given order in of the Bessel functions. The propagation constants identifying these modes are given by

$$
\beta_{m b}=j \sqrt{k^{2}-v_{m n}^{2}}, m=0,1,2, \ldots, n=1,2,3, \ldots .
$$

These satisfy the condition that $\beta^{2}<0$ for all propagating modes within the coaxial line. 
The second lowest mode in the typical ideal coaxial line is the $\mathrm{TE}_{11}$ mode. It has an approximate cutoff frequency described by [80]

$$
f_{c}=\frac{c}{\pi(a+b) \sqrt{\varepsilon_{r}}}
$$

for a dielectric with $\mu=\mu_{0}$. Equation (A-13) approximates the cutoff at a point where the modulation balf-wavelength equals the circumference of a circle midway between $\rho=a$ and $\rho=b$.

Coaxial transmission lines experience some attenuation doe to conductor and dielectric losses. Contrary to the boundary condition originally stated, the metal coax walls are not infinitely conducting, but have a conductivity, $\sigma$. The propagating wave penetrates into the conductors as defined by the skin depth

$$
\delta^{\prime}=\sqrt{\frac{2}{\cos \mu}}
$$

The conductor losses of the coaxial line given in $\mathrm{dB}$ per onit length are [80]

$$
\alpha_{c}=13.6 \frac{\delta^{\prime} \mu}{\lambda} \frac{1}{b}\left(1+\frac{b}{a}\right) \frac{\sqrt{\varepsilon_{x}}}{\ln (b / a)} \text {, }
$$

where $\lambda$ is the free space wavelength.

Dielectric losses arise from the finite time of molecular reorientation in the insulating materials [71]. Absorption is treated mathematically by assuming a complex relative permittivity

$$
\varepsilon_{\mathrm{I}}=\varepsilon^{\prime}+\mathrm{j} \varepsilon^{\prime \prime}
$$


Since the waveguide propagation constant is expressed as

$$
\beta=j k=j \omega \sqrt{\mu \varepsilon},
$$

an attenuation factor results from the imaginary part of $k$, while the real component gives rise to the material dispersion. An expression for the dielectric loss given in $\mathrm{AB}$ per unit length is [80]

$$
\alpha_{\mathrm{D}}=27.3 \frac{\sqrt{\varepsilon_{\mathrm{r}}}}{\lambda} \tan \delta
$$

where

$$
\tan \delta=\frac{\varepsilon^{\prime \prime}}{\varepsilon^{\prime}}
$$

is the loss tangent published in the handbooks for many dielectric materials.

Another useful property of coaxial lines is the maximum power-handling capability. This property is based on the dielectric standoff or breakdown electric field, $\mathrm{E}_{\mathrm{m}}$. The maximum power, in Watts, is given by

$$
P_{\max }=\frac{E_{m}^{2} b^{2}}{60} \frac{\ln (b / a)}{(b / a)^{2}}
$$

As a reference, the breakdown electric field for air is $-30 \mathrm{kV} / \mathrm{cm}$.

\section{Coaxial Airlines}

The design of expanded coaxial airlines is straightforward; most are intended for use with $50 \Omega R F$ test instrumentation. If $Z_{0}=50$ is substituted into $\mathrm{Eq}$. (A-11), the airline design requires that $b / a=2.301$. Equation $(A-13)$ for the higher order mode 
(HOM) cutoff frequency then simplifies to $f_{\mathrm{c}} \approx 2.89 / \mathrm{a} \mathrm{GHz}$, if the center conductor radius is given in $\mathrm{cm}$. For a desired HOM cutoff frequency in the cell design, the remaining geometric parameters can be determined. If a given working volume size is desired for testing a fiber coil, the TEM mode parameters can be computed.

A transition region in the cells is necessary to expand the radial dimensions from the input and output connectors to a reasonably large working volume. This is accomplished using tapered conical conducting sections. Conical launchers for coaxial transmission lines are common, and the impedance and feld matching conditions required for geometrical transitions have been well studied [8I]. The characteristic line impedance for the TEM mode in the tapered section is given by

$$
\mathrm{z}_{0}=\frac{\eta}{2 \pi} \ln \left[\frac{\tan \left(\theta_{2} / 2\right)}{\tan \left(\theta_{1 / 2}\right)}\right],
$$

where $\theta_{1}$ and $\theta_{2}$ are the taper angles of the center and outer conductors. If the angles are kept small, Eq. (A-21) reduces to Eq. (A-10) for a coaxial line of the appropriate geometry. Subsequently, cell design can be simplified by allowing the conductor radii ratto to be 2.3 throughout the cell length, even in the conical region. For an airline, this cylindrical-geometry-only approach can be used quite effectively to large taper angles. If $\theta_{2}$ does not exceed $45^{\circ}$, the characteristic impedance of the conical transition deviates less than $5 \%$ from $50 \Omega$.

Long tapered transition regions for the coaxial cells ensure good impedance matching in the lines; however, all field distarbances cannot be avoided at the geometrical discontinuities. At the discontinuities, the spherical wavefront of the conical section must make the transition to the cylindrical wavefront (planar) of the coax section [81]. Cell higher order modes are excited at the discontinuities, but they are 
evanescent, carrying no energy and dying out rapidly. Attennation to HOM propagation at the fregaencies, $f$, near cutoff is given in $d B$ per unit length by [80]

$$
\alpha_{m}=54.6 \frac{f_{c}}{c} \sqrt{1 \cdot\left(f / f_{c}\right)^{2}} .
$$

The effective cell bandwidths and the proximity of the fiber optic test coils in the working volume to the transition discontinoities can be determined from Eq. (A-22).

Three expanded airlines were designed and fabricated for testing of fiber current sensor coils. Table A-1 gives the dimensions and the HOM cutoff freguency for each airline. Each cell was machined from brass because of its workability, high electrical conductivity, and its relatively high chemical resistance. The cells were disassembled at mating joints for ease of placement of the fiber coils in the working volume. Polyurethane foam was used for all supports inside the cells, including most of the mandreis onto which the optical fiber coils were wound. The relative permittivity of the foam is -1.15 , which can perturb the cell impedance as much as $4.5 \Omega$ from its nominally $50 \Omega$ value. Use of the foam supports was thus limited to absolute minimum for operation.

All losses in the coaxial airlines are guite snali: dielectric loss is insignificant, and the conductor loss for the brass in $\mathrm{dB}$ per unit length is

$$
\alpha_{c}=2.25 \times 10^{-8} \frac{\sqrt{f}}{b} \text {. }
$$

For a frequency of $1 \mathrm{GHz}$ and a minimum value of $\mathrm{b}=0.49 \mathrm{~cm}$ at the cell connector transitions, this attenuation is only $0.0015 \mathrm{~dB} / \mathrm{cm}$. The maximam power handling capability of the airlines is $\mathrm{P}_{\max } \approx 2.36 \mathrm{~b}^{2}$ Megawatts if $\mathrm{b}$ is expressed in $\mathrm{cm}$. Again for the minimum value of $b$ at the connector transitions, $P_{\max }>500 \mathrm{~kW}$. 
The airline cell working volumes are of sufficient length to allow HOMs created at the transition discontinuities to be well damped. For the existing designs, Eq. (A-22)

Table A-1.

Expanded Coaxial Airline Cells

Cell Property

Cell outer dimensions

Total length

Cell Diameter

Outer conductor dimensions

Center section length

Center section ojameter

End section length

End section diameter at connector

End section taper angle
Cell \#1

Cel] \#2

[ dimensions given in inches and (cm) ]

Cell \#3

$18.000(45.72) \quad 24.000(60.96) \quad 6.000(15.24)$

$5.500(13.97) \quad 2.500(6.35) \quad 1.300(3.30)$

$6.000(15.24) \quad 18.000(45.72) \quad 4.820(12.24)$

$5.240(13.31) \quad 2.260(5.74) \quad 0.384(0.98)$

$5.410(13.74) \quad 2.410(6.12)$

$0.384(0.98) \quad 0.384(0.98)$

$26.86^{\circ} \quad 23.63^{\circ} \quad$ no taper

Inner conductor dimensions

Center section length

$6.000(15.24) \quad 18.000(45.72) \quad 4.820(12.24)$

Center section diameter

$$
2.280(5.79) \quad 0.980(2.49) \quad 0.167(0.42)
$$

End section length

$5.410(13.74) \quad 2.410(6.12)$

End section diameter at connector

$0.167(0.42)$

$0.167(0.42)$

End section taper angle

$12.28^{\circ}$

$10.64^{\circ}$

no taper

HOM cutoff frequency

$1.00 \mathrm{GHz} \quad 2.32 \mathrm{GHz} \quad 13.65 \mathrm{GHz}$ 
becomes

$$
\alpha_{m}=\frac{12.1}{b} \sqrt{1-\left(f / f_{c}\right)^{2}},
$$

with dimensions of $\mathrm{dB}$ per tunit length. For the largest outer conductor radius, $b=6.66$ $\mathrm{cm}$, evanescent modes are attenuated $1.2 \mathrm{~dB} / \mathrm{cm}$ at a frequency, $\mathrm{f}=0.75 \mathrm{f}_{\mathrm{c}}$. Depending on the perturbations created by the fiber coils inside the cell, operations to greater than $80 \%$ of the HOM cutoff freguency are predicted for all airlines.

The exact, aseful operating ranges are established through experimental performance verification. Part of the evaluation criteria cornes through measurement of basic properties of the cell using automatic network analysis. The transmission $\left(S_{31}\right)$ and reflection $\left(S_{11}\right)$ scattering parameters, both magnitude and phase, are collected for the cells in their final configurations. Along with these parameters the standing wave ratio (SWR) and the device impedance are computed from

$$
S W R=\frac{1+\left|S_{11}\right|}{1-\left|S_{11}\right|},
$$

and

$$
\mathrm{z}=\mathrm{z}_{0} \frac{1+\mathrm{s}_{11}}{1-\mathrm{S}_{11}} \text {, }
$$

where $Z, Z_{0}$, and $S_{11}$ are complex quantities. Ample information is provided from these electromagnetic characterizations to determine the range of useful freguencies for the testing of fiber sensor coils. 


\section{APPENDIX B \\ EXACT TREATMENT OF FIBER COIL LEADS}

The fiber leads on a Faraday effect cnrent sensor coil can significantly change the high frequency characteristics of the sensor response fanction. Each lead adds to the total fiber length in the coil, reducing the sensor bandwidth. More importantly, the position of the fiber leads creates a complex phase interaction between the propagating light in the fiber and the oscillating magnetic field signal. This produces deviations in the Faraday rotation from the simple response function for the centered, circular coil as described by $\mathrm{Eq}$. (5*2).

\section{Lead Effect Modis}

A simple model for a fiber coil lead is developed to allow for an analytical investigation of the effect on sensor response. As illastrated in Fig. B-1, the coil lead is treated as a constant radius arc of optical fiber originating a distance $\mathrm{X}$ from the coil edge and ending at a point on the coil proper tangent to jts curvature. Beyond the distance $\mathrm{X}$, the fiber lead is assumed perpendicular to the magnetic field and no Faraday interaction occors (B $\cdot d l=0$ ). The formulation in chapter 3 is tsed to derive the frequency dependent Faraday rotation for the fiber lead, $\Theta_{1}(\omega)$. Starting with Eg. (3-4), the angle $\psi$ is set to zero and integration occurs only over the fiber lead length, $\ell_{1}$, the distance between points 0 and 1 in Fig. B-1. The Faraday rotation is then

$$
\theta_{1}(\omega)=v \frac{\mu_{\mathrm{f}} \mathrm{i}}{2 \pi} \int_{0}^{\ell_{1}} \frac{\cos \psi}{\mathrm{r}} \mathrm{e}^{-\mathrm{j} \omega \mathrm{t}} \mathrm{d}
$$

Since both $\gamma$ and $\mathbf{r}$ depend on the position of the light in the fiber lead, both remain as part of the integrand. Changing the integral to one over time yields the expression 


$$
\Theta_{1}(\omega)=v \frac{\mu_{\mathrm{f}} \mathfrak{i}}{2 \pi}\left(\frac{n}{c}\right) \int_{0}^{t_{1}} \frac{\cos [\gamma(t)]}{I(t)} e^{-j \omega t} d t
$$

where $t_{1}$ is the light transit time throngh the lead section.

From the geometry in Fig. B-1, the following properties are derived:

$$
\gamma=\alpha^{\prime}+\beta^{\prime},
$$

$$
\sin \alpha^{\prime}=\frac{R_{\mathrm{I}}}{r} \sin \beta^{\prime},
$$

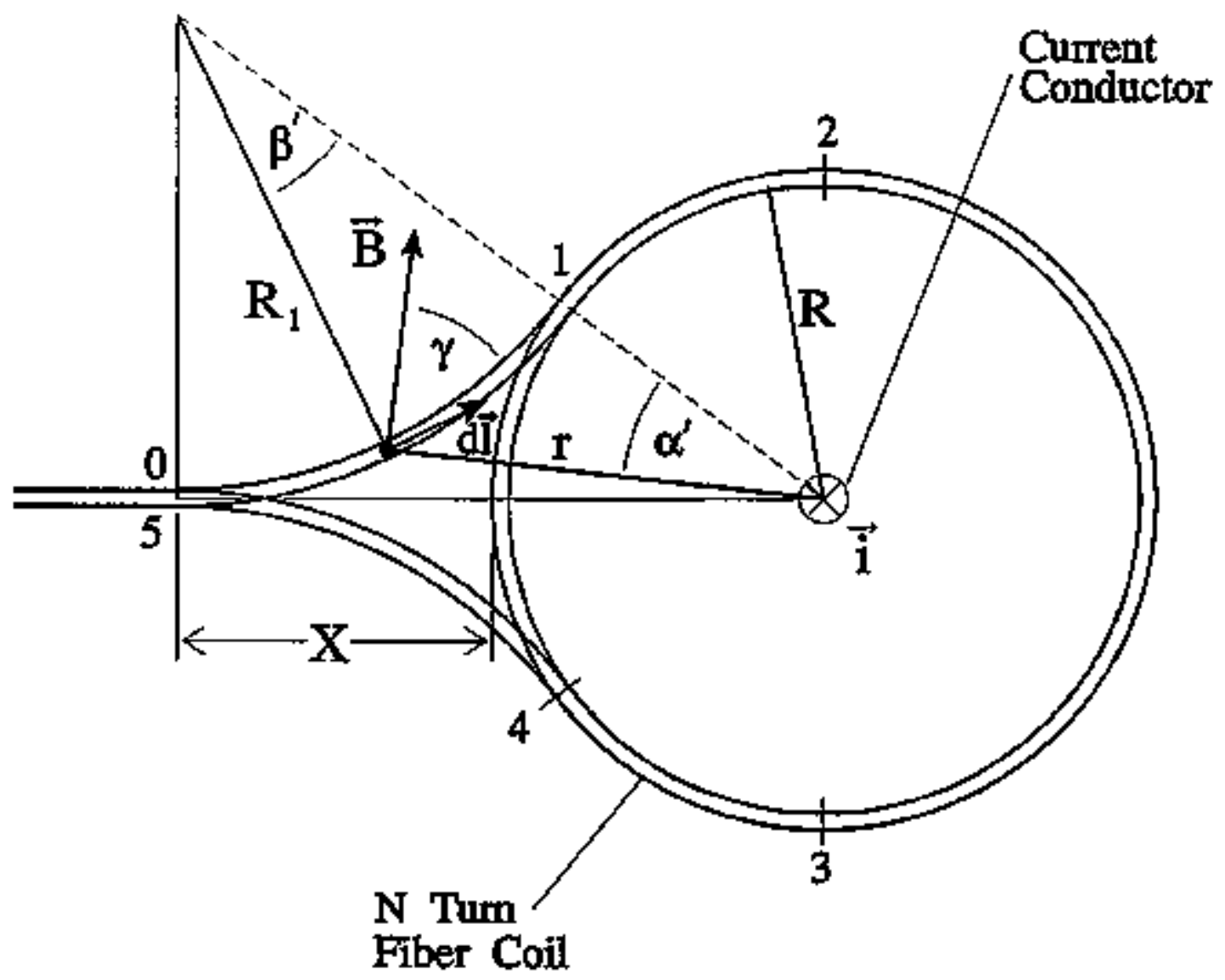

Fig. B-1. A geometrical model of the fiber leads on a current sensor coil. 


$$
\begin{gathered}
r^{2}=\left(R+R_{1}\right)^{2}+R_{1}^{2}-2 R_{1}\left(R+R_{I}\right) \cos \beta^{\prime}, \\
R_{1}=\frac{X^{2}}{2 R} \div X,
\end{gathered}
$$

and

$$
\beta_{\max }^{\prime}=\tan ^{-1}\left(\frac{1+R / x}{1+x / 2 R}\right) .
$$

The relationships between the angles and the light propagation times are

$$
\mathrm{t}_{1}=\frac{\mathrm{nR}}{\mathrm{c}} \beta_{\max }^{\prime}
$$

and

$$
\beta^{\prime}(t)=\frac{c}{n R_{1}}\left(t_{1}-t\right)
$$

A time dependent function for $r$ is constructed from Eqs. (B-5) and (B-9):

$$
r(t)=\sqrt{\left(R+R_{1}\right)^{2}+R_{1}^{2}-2 R_{1}\left(R+R_{1}\right) \cos \left[\frac{c}{a R_{1}}\left(t_{1}-t\right)\right]} .
$$

A sinitar expression for $\gamma$ is derived from Eqs. (B-3), (B-4), (B-5) and (B-9):

$$
\gamma(t)=\sin ^{-1}\left(\frac{R_{1} \sin \left[\frac{c}{n R_{1}}\left(t_{1}-t\right)\right]}{\sqrt{\left(R+R_{1}\right)^{2}+R_{1}^{2}-2 R_{1}\left(R+R_{1}\right) \cos \left[\frac{c}{a R_{1}}\left(t_{1}-t\right)\right]}}\right)+\frac{c}{n R_{1}}\left(t_{1}-t\right) \text {. }
$$

The exact coil lead Faraday rotation is found by substituting Eqs. (B-10) and (B-11) into Eq. (B-2). This final expression is 
$\theta_{1}(\omega)=v \frac{\mu_{f}}{2 \pi}\left(\frac{n}{c}\right) \int_{0}^{t_{1}} \frac{\left.\cos \left[\sin ^{-1} \sqrt{\sqrt{A_{1} \cdot A_{2} \cos \left[\frac{c}{n R_{1}}\left(t_{1}-t\right)\right]}}\right]+\frac{c}{n R_{1}}\left(t_{1}-t\right)\right]}{\sqrt{A_{1} \cdot A_{2} \cos \left[\frac{c}{n R_{I}}\left(t_{1}-t\right)\right]}} e^{-j \omega t} d t$,

where

$$
A_{1}=\left(R \div R_{1}\right)^{2}+R_{1}^{2}
$$

and

$$
A_{2}=2 R_{1}\left(R+R_{1}\right)
$$

Equations (B-12) must be integrated numerically to find the solution.

Input and output leads contribute differently to the Faraday interaction, although each can be treated with similar mathematics. Two fiber coil lead configurations have been used in this study, and both can be visualized osing the layout shown in Fig. B-1. In the first configuration, the light in the fiber propagates through the input lead from position 0 to position 1 , through an input transition section to position 2 , around $(\mathrm{N}-1 / 2)$ coil turns to position 3, through an output transition section to position 4 , and finally along an output lead to position 5. Light propagation in the second configuration differs only in that it passes through a complete $\mathrm{N}$ coil turns between positions 2 and 3. This means that positions 2 and 3 are coincident on the coil and the output fiber lead exits to the right of the coil in Fig. B-1, opposite that of the input lead. The interaction of the magnetic field with the light as it passes through the outpot transition section and output lead is identical for the two configurations except for a phase delay created by the extra half tum in the second configuration.

In the mathematical model, Eg. (B-12) describes the Faraday rotation for the input lead. A similar expression integrating from $t_{4}$ to $t_{5}$ (propagation times 
corresponding to light at positions 4 and 5 in Fig. B-1) describes the rotation for the ostput lead:

$\theta_{5}(\omega)=v \frac{\mu_{F} i}{2 \pi}\left(\frac{n}{c}\right) \int_{t_{4}}^{t_{s}} \frac{\cos \left[\sin ^{-1}\left(\frac{R_{t} \sin \left[\frac{c}{n R_{1}}\left(t-t_{4}\right)\right]}{\sqrt{A_{1}-A_{2} \cos \left[\frac{c}{B R_{1}}\left(t-t_{4}\right)\right]}}\right)+\frac{c}{n R_{1}}\left(t-t_{4}\right)\right]}{\sqrt{A_{1}-A_{2} \cos \left[\frac{c}{\pi R_{i}}\left(t-t_{4}\right)\right]}} e^{-j \omega t} d t$.

Between positions 1 and 4, the fiber follows the constant radius path of the coil proper. The expression for the rotation in this coil section then follows directly from Eqs. (5-1) and (5-2) and is given by

$$
\theta_{2,3,4}(\omega)=v \frac{\mu_{f}}{2 \pi}\left(\frac{\eta}{c}\right) \frac{\left(t_{4}-t_{5}\right)}{R}\left[\frac{\sin \left(\frac{\omega\left(t_{4}-t_{1}\right)}{2}\right)}{\left(\frac{\omega\left(t_{4}-t_{1}\right)}{2}\right)}\right] e^{-j \omega \frac{\left(t_{4}-t_{1}\right)}{2}} e^{-j \omega\left(t_{1} / 2\right)} .
$$

In general, the rotation expressed in Eq. (B-14) can be treated as a single quantity as shown. However, in some configurations it may be necessary to break ont the rotation into constituent components $\Theta_{2}(\omega), \Theta_{3}(\omega)$, and $\Theta_{4}(\omega)$, with corresponding separation in the time intervals $\left(t_{2}-t_{1}\right),\left(t_{3}-t_{2}\right)$, and $\left(t_{4}-t_{3}\right)$. One such configaration is the travelling wave fiber design where the coil proper and the lead transition sections may differ. The critical times corresponding to positions 1 through 4 shown in Fig. B-1 can be found from the associated geometries. These are expressed by

$$
t_{2}-t_{1}=t_{4}-t_{3}=\frac{n R}{c} \beta_{\max }^{\prime},
$$


and

$$
t_{3}-t_{2}=2 \pi R \frac{n}{c}\left\{\begin{array}{c}
(N-1 / 2) \\
\text { or }
\end{array}\right\} \text {. }
$$

The choice of the coil tum factor $(\mathrm{N}-1 / 2$ or $\mathrm{N}$ ) in Eq. (B-16) is dependent on the desired coil confüguration.

The time dependent expressions Egs. (B-12), (B-13) and (B-14) are added to detemine the total Faraday rotation as the light propagates from one end of the fiber sensor to the other. Since the complete model consists of several integral and nonintegral expressions, each for a distinct section of the coil, care must be taken to properiy nomalize to the low frequency response. In its final form the description of the relative Faraday rotation for the entire sensor is

$$
\frac{\Theta(\omega)}{\Theta_{0}}=\frac{\Theta_{1}(\omega)+\left(\Theta_{2}(0)+\ldots\right)+\Theta_{5}(\omega)}{\Theta_{1}(0)+\left(\Theta_{2}(0)+\ldots\right)+\Theta_{5}(0)} .
$$

Appropriate use of Eg. (B-14) or the constituent rotations is determined by the selected sensor configuration.

\section{Model Results and Comparisons}

The effects of fiber leads on the high frequency Faraday response of current sensor coils is dependent on the number of tums in the coil. Perturbations are at a maximum for a single turn coil, with effects duninishing as $\mathrm{N}$ becomes larger. Plotted in Fig. B-2 are the relative magnitudes of the Faraday rotation for one tum coils with the two lead configurations described previously. Lead distance is $X=R$. The resulting lead length is such that its radius of curvature is $R_{1}=1.5 \mathrm{R}$, which satisfies a condition that 
bends in the lead not produce a linear birefringence per unit length greater than that of the bends in the coil.

Corve (a) in Fig. B-2 is the frequency response for a coil with leads entering and exiting from the same side. This configuration is simply a single loop around the conductor. Its response matches closely that of the one turn leadless, centered and circular coil. The cutoff frequency is identical, but the $3 \mathrm{~dB}$ bandwidth is reduced slightly, approximately $1.5 \%$. A noticeable reduction in peak magnitude of the second response lobe (near $\omega \tau / 2=1.5 \pi$ ) is observed when the lead effects are included. This loss of response becomes more pronounced for each successively higher freguency labe

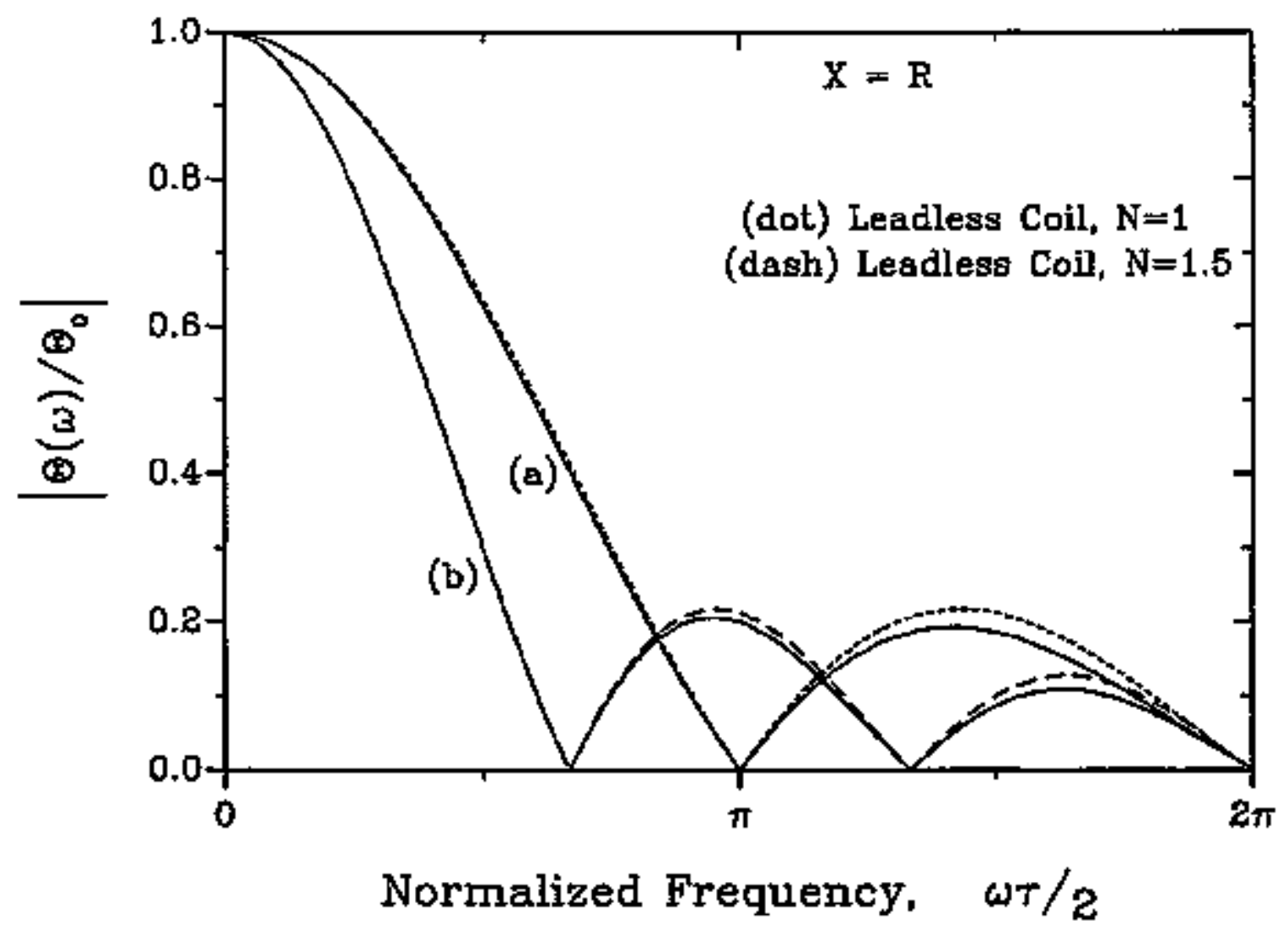

Fig. B-2. The predicted frequency response for a single turn Faraday effect fiber current sensor with (a) input and output leads exiting from the sante side of the coil and (b) the input and output leads exiting in opposite directions (equivalent to 1.5 coil toms). 
(not displayed in Fig. B-2), indicating that magnetic field phasing with the light propagating in the coil leads becomes much more important as frequency increases. As the number of turns in the coil is increased, the leads are less of a perturbation to the system frequency response. For as few as three coil tarns, the bandwidth and secondary lobe magnitudes are predicted to be essentially the same as those for the leadless coil.

Curve (b) in Fig. B-2 is the response for a single loop sensor with the fiber leads exiting the coil in opposite directions. This configuration has an effective 1.5 coil turns, thus making the bandwidth for curve (b) much snaller than for curve (a). The magnitude of the frequency response for curve (b) can be predicted quite well using Eq. (5-3) for a centered, circular coil with $\mathrm{N}=1.5$ (see the comparison in Fig. B-2). Deviations in response between the leadiess coil and the coil with leads occur only near the frequency peaks of the secondary lobes where a loss in magnitude is observed. Again, as the number of coil turns increases, this lead-induced response loss diminishes rapidly. However, it is necessary to include the extra half turn of fiber length when predicting frequency response for coils with this lead configuration remains unless $\mathrm{N}$ is an extremely large number. This is evident in comparing the measured and predicted values in Fig. 5-2 where $\mathrm{N}=10$.

In addition to the magnitude predictions, phase angle deviations are also considered in the lead effect model. For both configurations investigated, the coil leads add some length to the total fiber reguired in sensor fabrication. Thus, a propagation phase delay, compared to the leadless coil, is prodaced due to the slightly Ionger light transit time. This phase delay as a function of frequency follows exactly that given by Eq. (5-4) for the appropriate value of $\tau$.

The actual length of the fiber coil leads can have an impact on the Faraday frequency response. This is shown in Fig. B-3 for a single turn coil with leads exiting from the same side of the coil and with $X=10 R$. Note that the added fiber length in the leads results in a loss of $3 \mathrm{~dB}$ bandwidth, but that the cutoff frequency of the curve is 


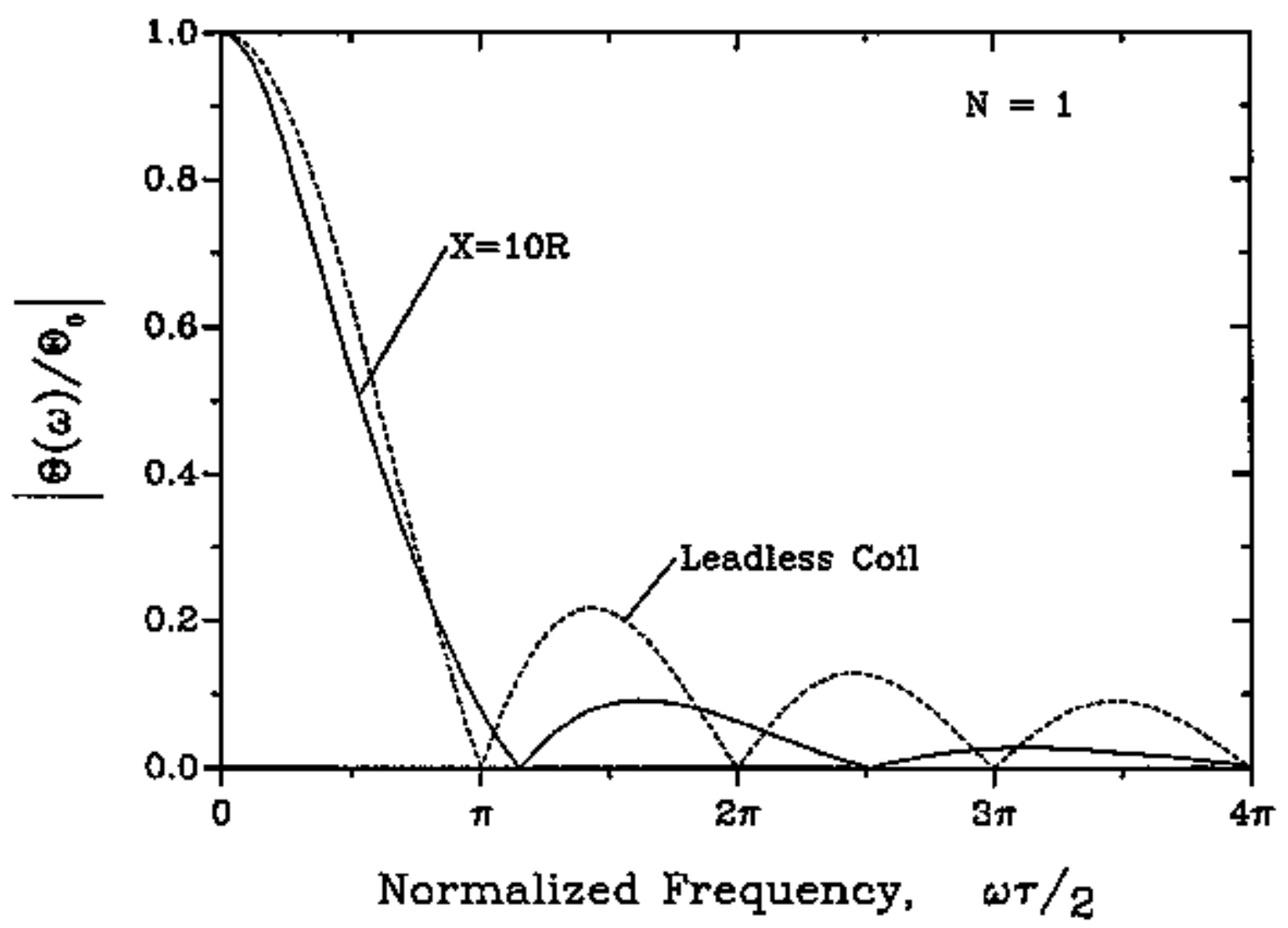

Fig. B-3. The effect of lead length on the relative response magnitude of a single turn fiber current sensor.

shifted to a higher value than that for the leadless coil. This feature occurs because light propagating in the fiber lead experiences a much weaker Faraday interaction - the angle $\gamma$ is large when $\mathrm{X}$ is large - than in the coil proper. At the low freguencies where the magnetic field polarization is changing slowly, the entire fiber length, including the long leads, contributes to the response. But at the higher frequencies $(\omega \tau / 2>0.6 \pi)$, where the magnetic field wavelength is approximately the same as the length of the fiber in the leadless coil, the leads contribute less to the total response and an effective shortening of the interaction length results. The decreasing $d B$ and increasing $f_{c o}$ continues to occur for the single tum coil as more length is added to the leads. Eventually, the leads become so long that total Faraday interaction in them is comparatively weak. At that point the 
model begins to exhibit anomalous resulis due to the segmented mathenatical treatment of the different ftber sections. All of the predicted response then derives from the remaining half-turn of the coil proper, with non-interactive delays due to the leads; a delta function-type response results.

If the model allowed, it wonld be of theoretical interest to explore more fully the extreme limit, $X \rightarrow \infty$, for the long leats on a single furn fiber current sensor coil. In experimental coils, however, such long lead lengths are simply not practical, especially if the stringent fiber geometries are followed as required by the model. True interaction lengths in the coil leads will always be significantly shorter. Thus, frequency response shifts due to long fiber leads are not expected to be greater than those shown in Fig. B-3 for $\mathrm{X}=10 \mathrm{R}$. And, as mentioned previously, the lead effects become less pronounced as the number of turns in the fiber coil increases. For $N \geq 10$, the Faraday response can be predicted guite precisely by the leadless coil model, regardless of the ffber coil lead lengths. 


\section{APPENDLX C DERIVATION OF EFFECTIVE RELATIVE PERMITTIVTTY}

The cross sectional geometry of the multilayer delectric coaxial line is defined by Fig. C.1. Inner conductor radius is ' $a$ ', outer conductor radius is ' $b$ ', and the dielectric transitions occur at $r_{1}$ and $I_{2}$. The relative permittivities at radii $\rho$ are given as $\varepsilon_{1}$ for $a<$ $\rho<r_{1}$ and $r_{2}<\rho<b$, and $\varepsilon_{2}$ for $r_{1}<\rho<r_{2}$. At low frequencies, the fields in all the dielectric regions are assnmed to be TEM. The radial electric field between a and $b$ then has a $1 / \rho$ dependency so that in medium $1, E_{1}=C_{1} / \rho$, and in medium $2, E_{2}=C_{2} / \rho$, where

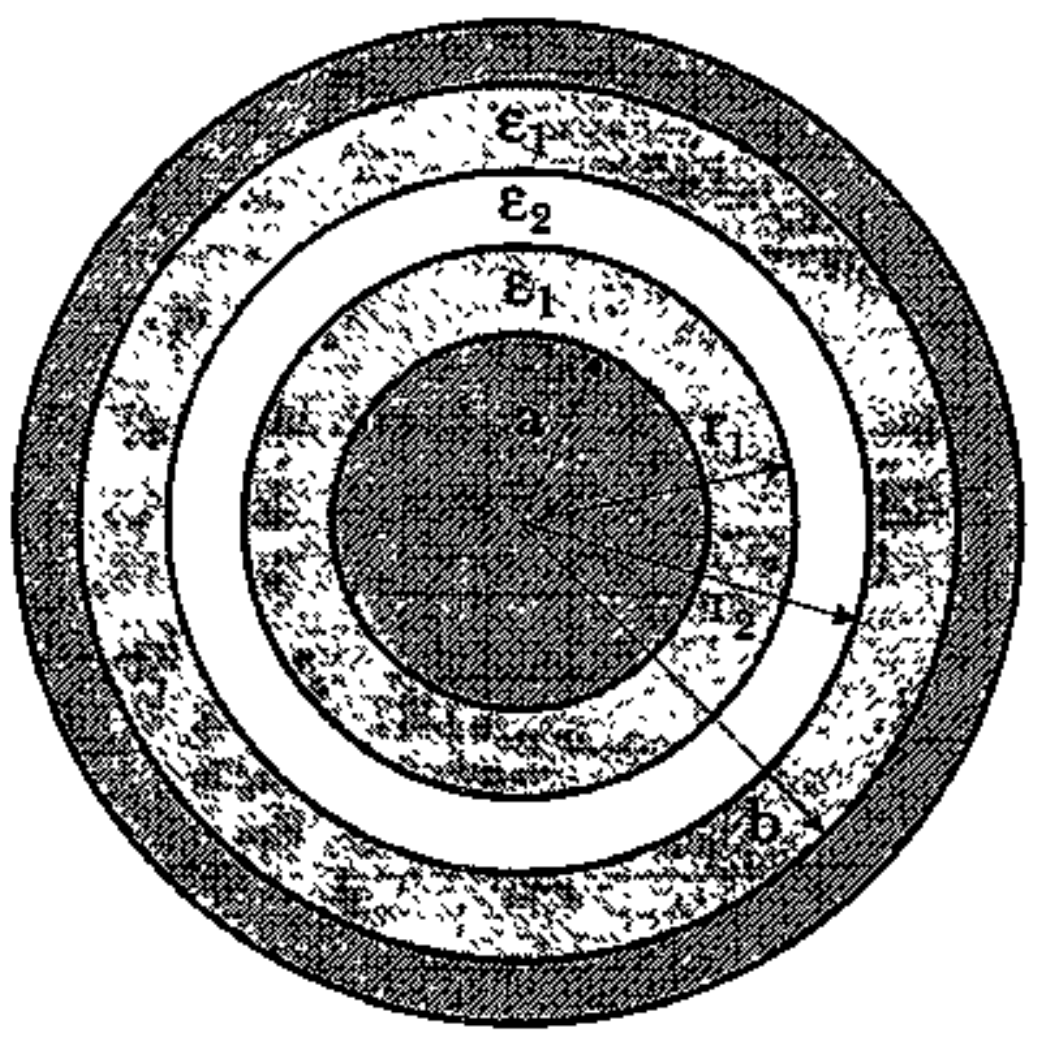

Fig. C-1. Cross section of the multilayer dielectric coaxial line. A medium with relative permittivity $\varepsilon_{2}$ is surrounded by a medium of relative permittivity $\varepsilon_{1}$. Coax conductors are defined by inner radias a and outer radius $b$. 
$C_{l}$ and $C_{2}$ are constants. An equivalent effective (homogeneous) mediam is defined throughout the dielectric region such that $\mathrm{E}_{\mathrm{eff}}=\mathrm{C}_{\text {eff }} / \mathrm{p}$ for $\mathrm{a}<\rho<\mathrm{b}$. The electric field is assumed to vanish at the conductor walis $(\sigma=\infty)$, and the boundary condition $\mathbf{D}_{\mathbf{1}} \bullet \mathbf{n}=$ $D_{2} \cdot n$ is applied at $r_{1}$ and $r_{2}$. An effective displacement vector, $D_{\text {eff }}$ is defined so that in the coaxial dielectric volume their exists an equivalent medium with relative perrititivity, $\varepsilon_{\text {efe }}$, and $D_{\text {eff }}=\varepsilon_{\text {eff }} \mathbf{E}_{\text {eff }}$

The voltage between the conductors in the coaxial cell is found from the line integral over the radial component of the electric field,

$$
v_{a b}=\int_{a}^{b} E_{\rho} d \rho
$$

It follows then that

$$
V_{a b}=\int_{a}^{b}\left(E_{\text {eff }}\right)_{\rho} d \rho
$$

and equivalently

$$
V_{a b}=\int_{a}^{I_{1}} E_{1} d \rho+\int_{r_{1}}^{r_{2}} E_{2} d \rho+\int_{r_{2}}^{b} E_{1} d \rho .
$$

Equation (C-2) reduces to

$$
v_{a b}=C_{e f f} \int_{a}^{b} \frac{1}{\rho} d \rho=C_{e f f} \ln \left(\frac{b}{a}\right)
$$

To solve Eq. (C-3), the boundary condition at the dielectric interface is applied so that

$$
\mathrm{E}_{2}=\frac{\varepsilon_{1}}{\varepsilon_{2}} \mathrm{E}_{1}
$$


Then substituting for $E_{1}$ and $E_{2}$ in Eq. (C-3),

$$
V_{a b}=C_{1}\left[\int_{a}^{T_{1}} \frac{1}{\rho} d \rho+\frac{\varepsilon_{1}}{\varepsilon_{2}} \int_{I_{1}}^{T_{2}} \frac{1}{\rho} d \rho+\int_{r_{2}}^{b} \frac{1}{p} d \rho\right]
$$

This integral expression has the solution

$$
\mathrm{V}_{\mathrm{ab}}=C_{1}\left[\ln \left(\frac{\mathrm{r}_{1}}{\mathrm{a}}\right)+\frac{\varepsilon_{1}}{\varepsilon_{2}} \ln \left(\frac{\mathrm{r}_{2}}{\mathrm{I}_{1}}\right)+\ln \left(\frac{\mathrm{b}}{\mathrm{r}_{2}}\right)\right] .
$$

Equation (C-7) can be further reouced to

$$
v_{a b}=C_{1}\left[\ln \left(\frac{b}{a}\right)+\left(\frac{\varepsilon_{1}}{\varepsilon_{2}}-1\right) \ln \left(\frac{r_{2}}{r_{1}}\right)\right] .
$$

Combining Eqs. (C-4) and (C-8) gives

$$
\frac{C_{\text {eff }}}{C_{1}}=\frac{\left[\ln \left(\frac{b}{a}\right)+\left(\frac{E_{1}}{E_{2}} \cdot 1\right) \ln \left(\frac{r_{2}}{r_{1}}\right)\right]}{\ln \left(\frac{b}{a}\right)}
$$

or

$$
\frac{C_{\text {eff }}}{C_{1}}=\left[1+\left(\frac{\varepsilon_{1}}{\varepsilon_{2}}-1\right) \frac{\ln \left(\mathrm{I}_{2} / \mathrm{I}_{1}\right)}{\ln (\mathrm{b} / \mathrm{a})}\right]
$$

The energy density in the dielectric of the coaxial line is given by

$$
\frac{d W}{d V_{0}}=\frac{1}{2}(D \cdot E)
$$


which becomes $1 / 2 \varepsilon \mathrm{E}^{2}$ for a TEM mode. The total energy per unit length is found by integrating Eq. (C-11) over a given plangr surface, $S$, in the coaxial line dielectric region.

$$
\frac{d W}{d z}=\frac{1}{2} \varepsilon \int_{S} E^{2} d A
$$

Here $d A=\rho d \rho d \phi$. The electric field for the TEM mode is azimuthally symmetric (no $\phi$ dependency). Then for a radially dependent electric field in a homogeneous dielectric with an effective relative permittivity, Eq. (C-12) becomes

$$
\frac{\mathrm{dW}}{\mathrm{dz}}=\frac{I}{2} \varepsilon_{\text {eff }} C_{\mathrm{eff}}^{2} 2 \pi \int_{\mathrm{a}}^{\mathrm{b}}\left(\frac{\mathrm{I}}{\rho}\right)^{2} \rho \mathrm{d} \rho
$$

which has a solution of

$$
\frac{d W}{d z}=\pi \varepsilon_{\text {eff }} C_{\text {eff }}^{2} \ln \left(\frac{b}{a}\right)
$$

Equivalently, Eq. (C-12) can be broken into its constituent integrals for each of the three radial dielectric regions:

$$
\frac{d W}{d z}=\pi\left[c_{1}^{2} \varepsilon_{1} \int_{a}^{T_{1}}\left(\frac{1}{\rho}\right)^{2} \rho d \rho+C_{1}^{2} \varepsilon_{2} \int_{I_{1}}^{r_{2}}\left(\frac{\varepsilon_{1}}{\varepsilon_{2}} \frac{1}{\rho}\right)^{2} \rho d \rho+C_{1}^{2} \varepsilon_{1} \int_{r_{2}}^{b}\left(\frac{1}{\rho}\right)^{2} \rho d \rho\right]
$$

where Eq. (C-5) has been applied in the second dielectric. Then

$$
\frac{\mathrm{dW}}{\mathrm{dz}}=\pi \varepsilon_{1} \mathrm{C}_{1}^{2}\left[\ln \left(\frac{\mathrm{r}_{1}}{\mathrm{a}}\right)+\frac{\varepsilon_{1}}{\varepsilon_{2}} \ln \left(\frac{\mathrm{r}_{2}}{\mathrm{r}_{1}}\right)+\ln \left(\frac{\mathrm{b}}{\mathrm{r}_{2}}\right)\right] .
$$


This further reduces to

$$
\frac{d W}{d z}=\pi \varepsilon_{1} C_{1}^{2}\left[\ln \left(\frac{b}{a}\right)+\left(\frac{\varepsilon_{1}}{\varepsilon_{2}}-1\right) \ln \left(\frac{r_{2}}{r_{1}}\right)\right]
$$

Combining Eqs. (C-14) and C-17) leads to

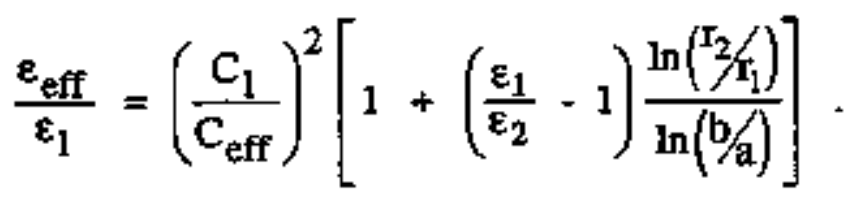

Finally, an effective relative permittivity results by substituting $C_{e f} / C_{1}$ from Eq. (C-10) into $\mathrm{Eg}+(\mathrm{C}-18)$. This gives

$$
\frac{\varepsilon_{\mathrm{eff}}}{\varepsilon_{1}}=\left[1+\left(\frac{\varepsilon_{1}}{\varepsilon_{2}} \cdot 1\right) \frac{\ln \left(\mathrm{I}_{2} / \mathrm{T}_{1}\right)}{\ln (\mathrm{b} / \mathrm{a})}\right]^{+1} .
$$

The ratio of the effective relative permittivity to the relative permittivity of medium $I$ is dependent only on the geometry of the coaxial line and the ratio of the two media relative permittivities. If medium 2 is very thin so that $r_{1} \approx I_{2}$, or if the two dielectrics have approximately the same relative permittivities, $\varepsilon_{1} \approx \varepsilon_{2}$, then $\varepsilon_{-f t} \rightarrow \varepsilon_{1}$ in Eq. (C-19) as expected. And if medium 2 is large so that it nearly fills the entire volume of the coaxial line, then $r_{1} \approx a, r_{2} \approx b$ and $\varepsilon_{e f f} \rightarrow \varepsilon_{2}$. 


\section{APPENDIX D \\ WA VEGUIDE MODES FOR AZMUUTHALEY SYMMETRYC \\ FIELDS IN A MULTILA YER COAXIAL TRANSMISSION \\ LINE WTTH COMPLEX RELATIVE PERMTTTIVITY}

In these discussions the wavegnide geometry and material dielectric properties for the coaxial line are taken as tescribed in Appendix $\mathrm{C}$ and as illostrated in Fig. C-1. Material wave parameters $k_{1,2}=(\omega / c) \sqrt{\varepsilon_{1,2}}$ and the waveguide propagation constant, $\beta_{\mathrm{mo}}$ as given by Eq. (A-8), are all treated as complex quantities. Dae to the added dielectric, medium 2, the electric and magnetic field components in all the coaxial dielectric layers may, in general, be coupled together, and defining strictly TE and TM modes as in the simple (single dielectric) coaxial line is not appropriate. The treatment of this coupled mode problem is quite involved mathematically, and thus a complete description of the transmission cell higher order modes is not attempted here. Instead, azimuthal symmetry $(m=0)$ is assumed, the field components become uncoupled, and TE and TM modes can be derived separately. The waveguide propagation constants, $\beta_{\text {mar }}$ for each of the modes then takes on the simpler form, $\beta_{0 a^{*}}$ The derivation of the modal eigenvalue equations in the following discussions is adapted from Wait [73].

\section{TManModes}

If m $=0$ in Eqs. (A-1), $E_{\phi}$ and $H_{p}$ vanish and the remaining field components $E_{z}$, $\mathrm{H}_{\phi}$ and $\mathrm{E}_{\rho}$ are sufficient to describe the TM fields in each coaxial layer. In the region defined by $a<\rho<r_{1}$, the TM field components are given by

$$
E_{2 l}=u_{1}^{2}\left[A_{1} J_{0}\left(u_{1} \rho\right)+B_{1} Y_{0}\left(u_{1} \rho\right)\right],
$$




$$
H_{\phi \Gamma}=-\frac{j k_{1}^{2}}{\mu \omega}\left[A_{1} \frac{\partial}{\partial \rho} J_{0}\left(n_{1} \rho\right)+B_{1} \frac{\partial}{\partial \rho} Y_{0}\left(u_{1} \rho\right)\right],
$$

and

$$
E_{\rho 1}=-\beta\left[A_{1} \frac{\partial}{\partial \rho} J_{0}\left(u_{1} \rho\right)+B_{1} \frac{\partial}{\partial \rho} Y_{0}\left(v_{1} \rho\right)\right] .
$$

Here

$$
\mathrm{u}_{\mathrm{l}}^{2}=\mathrm{k}_{1}^{2}+\mathrm{\beta}^{2} \text {. }
$$

In Eqs. (D-1) and (D-2), $u_{1}$ and $\beta$ are both multivalued parameters $-v_{1,0 \mathrm{a}}$ and $\beta_{0 \mathrm{n}}$ for $\mathrm{n}=$ $1,2,3 \ldots-$ dae to the waveguide modal structure. For simplicity in these derivations, the modal subscripts ' $0 \mathrm{n}$ ' are dropped from $\mathrm{u}$ and $\beta$, and the remaining numerical subscripts refer only to the dielectric region they represent.

In the region defined by $r_{2}<\rho<b$, the field components $E_{23}, H_{43}$ and $E_{p 3}$ will be identical to those described in Eqs. (D-1) only with $A_{1}$ and $B_{1}$ replaced with $A_{3}$ and $B_{3}$. For $r_{1}<\rho<r_{2}$, the TM field components are

$$
\begin{gathered}
\mathrm{E}_{\mathrm{z} 2}=\mathrm{u}_{2}^{2}\left[\mathrm{~A}_{2} \mathrm{~J}_{0}\left(\mathrm{u}_{2} \rho\right)+\mathrm{B}_{2} \mathrm{Y}_{0}\left(\mathrm{u}_{2} \rho\right)\right], \\
\mathrm{H}_{\phi 2}=-\frac{j \mathrm{k}_{2}^{2}}{\mu \omega}\left[\mathrm{A}_{2} \frac{\partial}{\partial \rho} \mathrm{J}_{0}\left(\mathrm{u}_{2} \rho\right)+\mathrm{B}_{2} \frac{\partial}{\partial \rho} Y_{0}\left(\mathrm{u}_{2} \rho\right)\right],
\end{gathered}
$$

and

$$
\mathbf{E}_{\rho z}=-\beta\left[A_{2} \frac{\partial}{\partial \rho} J_{0}\left(u_{2} \rho\right)+B_{2} \frac{\partial}{\partial \rho} Y_{0}\left(u_{2} \rho\right)\right],
$$

with

$$
u_{2}^{2}=k_{2}^{2}+\beta^{2} \text {. }
$$

The metal walls of the coaxisl line are considered to be infinitely conducting so that $E_{z}$ vanishes at $\rho=a$ and $\rho=b$. Then 


$$
\frac{A_{1}}{B_{1}}=-\frac{Y_{0}\left(u_{1} a\right)}{J_{0}\left(u_{1} a\right)}
$$

and

$$
\frac{A_{3}}{B_{3}}=-\frac{Y_{0}\left(u_{1} b\right)}{J_{0}\left(u_{1} b\right)}
$$

At the dielectric interfaces $\rho=r_{1}$ and $\rho=r_{2}$, the tangential conponents, $E_{2}$ and $H_{4}$ are continuous. At $\rho=r_{1}$, this leads to

$$
u_{1}^{2} B_{1}\left[\left(\frac{A_{i}}{B_{1}}\right) J_{0}\left(u_{1} r_{1}\right)+Y_{0}\left(u_{1} r_{i}\right)\right]=u_{2}^{2} B_{2}\left[\left(\frac{A_{2}}{B_{2}}\right) J_{0}\left(u_{2} r_{1}\right)+Y_{0}\left(u_{2} r_{1}\right)\right],
$$

and

$$
k_{1}^{2} u_{1} B_{1}\left[\left(\frac{A_{1}}{B_{1}}\right) J_{1}\left(u_{1} r_{1}\right)+Y_{1}\left(u_{1} r_{1}\right)\right]=k_{2}^{2} u_{2} B_{2}\left[\left(\frac{A_{2}}{B_{2}}\right) J_{t}\left(u_{2} r_{1}\right)+Y_{1}\left(u_{2} r_{i}\right)\right] .
$$

In Eq. (D-8), the property of the Bessel function

$$
\frac{\partial}{\partial \rho} \mathbf{J}_{0}(u \rho)=-\square \mathbf{J}_{1}(u \rho)
$$

has been applied. Equation (D-8) is divided by Eq. (D-7), and $A_{1} / B_{1}$ is replaced by its equivalent form from Eq. (D-5). The following expression results.

$$
\left(\frac{k_{1}^{2}}{u_{1}}\right)\left[\frac{Y_{1}\left(u_{1} r_{1}\right)-\frac{Y_{0}\left(u_{1} a\right)}{J_{0}\left(u_{1} a\right)} Y_{1}\left(u_{1} r_{1}\right)}{Y_{0}\left(u_{1} r_{1}\right)-\frac{Y_{0}\left(u_{1}^{a}\right)}{J_{0}\left(u_{1} a\right)} Y_{0}\left(u_{1} r_{1}\right)}\right]=\left(\frac{k_{2}^{2}}{u_{2}}\right)\left[\frac{\left(\frac{A_{2}}{B_{2}}\right) J_{1}\left(u_{2} r_{1}\right)+Y_{1}\left(u_{2} r_{1}\right)}{\left(\frac{A_{2}}{B_{2}}\right) J_{0}\left(u_{2} r_{1}\right)+Y_{0}\left(u_{2} r_{1}\right)}\right] .
$$


Solving for $\mathrm{A}_{2} / \mathrm{B}_{2}$ gives

$$
\frac{A_{2}}{B_{2}}=\frac{Y_{1}\left(u_{2} I_{1}\right)-\left(\frac{k_{1}}{k_{2}}\right)^{2} \frac{u_{2}}{u_{1}} \chi_{1} Y_{0}\left(u_{2} I_{1}\right)}{\left(\frac{k_{1}}{k_{2}}\right)^{2} \frac{u_{2}}{u_{1}} \chi_{1} J_{0}\left(u_{2} r_{1}\right)-J_{1}\left(u_{2} r_{1}\right)},
$$

where $\chi_{1}$ is the bracketed factor on the left side of Eq. (D-10) and is given by

$$
\chi_{1}=\frac{Y_{1}\left(u_{1} r_{1}\right)-\frac{Y_{0}\left(u_{1} a\right)}{J_{0}\left(u_{1} a\right)} J_{1}\left(u_{1} r_{1}\right)}{Y_{0}\left(u_{1} r_{l}\right)-\frac{Y_{0}\left(u_{1} a\right)}{J_{0}\left(u_{1} a\right)} J_{0}\left(u_{1} r_{1}\right)} .
$$

Al the second dielectric interface, $\rho=T_{2}$, a similar expression for $A_{2} / B_{2}$ is derived. It is given by

$$
\frac{A_{2}}{B_{2}}=\frac{Y_{1}\left(u_{2} r_{2}\right)-\left(\frac{k_{1}}{k_{2}}\right)^{2} \frac{u_{2}}{v_{1}} \chi_{2} Y_{0}\left(u_{2} I_{2}\right)}{\left(\frac{k_{1}}{k_{2}}\right)^{2} \frac{u_{2}}{u_{1}} \chi_{2} J_{0}\left(u_{2} I_{2}\right)-J_{1}\left(u_{2} r_{2}\right)},
$$

where

$$
X_{2}=\frac{Y_{1}\left(u_{1} I_{2}\right)-\frac{Y_{0}\left(u_{1} b\right)}{J_{0}\left(u_{1} b\right)} J_{1}\left(u_{1} I_{2}\right)}{Y_{0}\left(u_{1} I_{2}\right)-\frac{Y_{0}\left(u_{1} b\right)}{J_{0}\left(u_{1} b\right)} J_{0}\left(u_{1} I_{2}\right)} .
$$

Combining Eqs. (D-11) and (D-13) gives a final transcendental eigenvalue equation for the $\mathrm{TM}_{\mathrm{On}}$ modes, 


$$
\frac{\left(\frac{k_{1}}{k_{2}}\right)^{2} \frac{u_{2}}{u_{1}} \chi_{2} J_{0}\left(u_{2} r_{2}\right)-J_{1}\left(u_{2} r_{2}\right)}{\left(\frac{k_{1}}{k_{2}}\right)^{2} \frac{u_{2}}{u_{1}} \chi_{1} J_{0}\left(u_{2} r_{1}\right)-J_{1}\left(u_{2} r_{1}\right)}=\frac{\left(\frac{k_{1}}{k_{2}}\right)^{2} \frac{u_{2}}{u_{1}} \chi_{2} Y_{0}\left(u_{2} r_{2}\right)-Y_{1}\left(u_{2} r_{2}\right)}{\left(\frac{k_{1}}{k_{2}}\right)^{2} \frac{u_{2}}{u_{1}} \chi_{1} Y_{0}\left(u_{2} I_{1}\right)-Y_{1}\left(u_{2} r_{1}\right)},
$$

with $\chi_{1}$ and $\chi_{2}$ given by Eqs. (D-12) and (D-14).

\section{TEnModes}

In a fastion similar to the $\mathrm{TM}_{\mathrm{op}}$ mode derivations, the $\mathrm{TE}_{\mathrm{p}}$ modes of the waveguide are found from Eqs. (A-2) with $\mathrm{m}=0$. In the dielectric region defined by $\varepsilon_{1}$, the field components are given by

$$
\begin{gathered}
H_{z 1,3}=u_{1}^{2}\left[C_{1,3} J_{0}\left(u_{1} \rho\right)+D_{1,3} Y_{0}\left(u_{1} \rho\right)\right], \\
E_{\phi 1,3}=j \mu \omega\left[C_{1,3} \frac{\partial}{\partial \rho} J_{0}\left(u_{1} \rho\right)+D_{1,3} \frac{\partial}{\partial \rho} Y_{0}\left(u_{1} \rho\right)\right],
\end{gathered}
$$

and

$$
\mathrm{H}_{\rho \mathrm{L}, 3}=-\beta\left[\mathrm{C}_{1,3} \frac{\partial}{\partial \rho} \mathrm{J}_{0}\left(\mathrm{v}_{1} \rho\right)+\mathrm{D}_{1,3} \frac{\partial}{\partial \rho} \mathrm{Y}_{0}\left(\mathrm{u}_{\mathrm{t}} \rho\right)\right] \text {, }
$$

where the subscript 1 applies for $:<\rho<r_{1}$ and the subscript 3 applies for $r_{2}<p<b$. The TE field components for $r_{1}<\rho<r_{2}$, where the relative permittivity is $\varepsilon_{2}$, are given by

$$
\begin{gathered}
H_{Z_{2}}=u_{z}^{2}\left[C_{2} J_{0}\left(u_{2} \rho\right)+D_{2} Y_{0}\left(u_{2} \rho\right)\right] \\
E_{\phi 2}=j \mu \omega\left[C_{2} \frac{\partial}{\partial \rho} J_{0}\left(u_{2} \rho\right)+D_{2} \frac{\partial}{\partial \rho} Y_{0}\left(u_{2} \rho\right)\right],
\end{gathered}
$$

and

$$
H_{\rho 2}=-\beta\left[C_{2} \frac{\partial}{\partial \rho} J_{0}\left(u_{2} \rho\right)+D_{2} \frac{\partial}{\partial \rho} Y_{0}\left(u_{2} \rho\right)\right] .
$$


Again the Bessel function property, Eq. (D-9), can be applied to Egs. (D-16b), (D-16c), (D-17b), and (D-17c).

At the infinitely conducting metal walls, the tangential $E$ component must vanish. For these TE modes, $E_{4}=0$ at both $\rho=a$ and $\rho=b$. Then

$$
\frac{C_{1}}{D_{1}}=-\frac{Y_{1}\left(u_{1} a\right)}{J_{1}\left(u_{1} a\right)}
$$

and

$$
\frac{C_{3}}{D_{3}}=-\frac{Y_{1}\left(u_{1} b\right)}{J_{1}\left(u_{1} b\right)}
$$

At $\rho=r_{1}$, the tangential components $E_{\phi}$ and $H_{z}$ are continuous across the dielectric interface. Thus, for $\mathrm{H}_{2}$,

$$
u_{1}^{2} D_{1}\left[\left(\frac{C_{1}}{D_{1}}\right) J_{0}\left(u_{1} I_{1}\right)+Y_{0}\left(u_{1} r_{1}\right)\right]=u_{2}^{2} D_{2}\left[\left(\frac{C_{2}}{D_{2}}\right) J_{0}\left(u_{2} r_{1}\right)+Y_{0}\left(u_{2} r_{1}\right)\right],
$$

and for $E_{\phi}$,

$$
\mathrm{v}_{1} \mathrm{D}_{1}\left[\left(\frac{\mathrm{C}_{1}}{\mathrm{D}_{1}}\right) \mathrm{J}_{1}\left(\mathrm{u}_{1} \mathrm{r}_{1}\right)+\mathrm{Y}_{1}\left(\mathrm{u}_{1} \mathrm{r}_{1}\right)\right]=\mathrm{v}_{2} \mathrm{D}_{2}\left[\left(\frac{\mathrm{C}_{2}}{\mathrm{D}_{2}}\right) \mathrm{J}_{1}\left(\mathrm{u}_{2} \mathrm{r}_{1}\right)+\mathrm{Y}_{1}\left(\mathrm{u}_{2} \mathrm{r}_{1}\right)\right] .
$$

Using Eq. (D-18) to eliminate $C_{1} / D_{1}$, and dividing Eq. (D-21) by Eq. (D-20) yields

$$
\frac{1}{u_{1}}\left[\frac{Y_{1}\left(u_{1} r_{1}\right)-\frac{Y_{1}\left(u_{1} a\right)}{J_{1}\left(u_{1} a\right)} J_{1}\left(u_{1} r_{2}\right)}{Y_{0}\left(u_{1} r_{1}\right)-\frac{Y_{1}\left(u_{1} a\right)}{J_{1}\left(u_{1} a\right)} J_{0}\left(u_{1} r_{1}\right)}\right]=\frac{1}{u_{2}}\left[\frac{\left(\frac{C_{2}}{D_{2}}\right) J_{1}\left(u_{2} r_{1}\right)+Y_{1}\left(u_{2} r_{1}\right)}{\left(\frac{C_{2}}{D_{2}}\right) J_{0}\left(u_{2} r_{1}\right)+Y_{0}\left(u_{2} r_{1}\right)}\right] .
$$

Then solving for $\mathrm{C}_{2} / \mathrm{D}_{2}$ gives 


$$
\frac{C_{2}}{D_{2}}=\frac{Y_{1}\left(u_{2} r_{1}\right)-\frac{u_{2}}{u_{1}} Z_{1} Y_{0}\left(u_{2} r_{1}\right)}{\frac{u_{2}}{u_{1}} Z_{1} J_{0}\left(u_{2} r_{1}\right)-J_{1}\left(u_{2} r_{1}\right)},
$$

where $Z_{1}$ is the factor in the brackets on the left side of Eq. (D-22) and is given by

$$
Z_{1}=\frac{Y_{1}\left(u_{1} r_{1}\right)-\frac{Y_{1}\left(u_{1} a\right)}{J_{1}\left(u_{1} a\right)} J_{1}\left(u_{1} r_{j}\right)}{Y_{0}\left(u_{1} r_{1}\right)-\frac{Y_{1}\left(u_{1} a\right)}{J_{1}\left(u_{1} a\right)} J_{0}\left(u_{1} r_{1}\right)} .
$$

At the second dielectric interface, $\rho=r_{2}$, the tangential field components are also continuous, which leads to a similar expression for $\mathrm{C}_{2} / \mathrm{D}_{2}$ given by

$$
\frac{C_{2}}{D_{2}}=\frac{Y_{1}\left(u_{2} r_{2}\right)-\frac{u_{2}}{u_{1}} Z_{2} Y_{0}\left(u_{2} I_{2}\right)}{\frac{u_{2}}{u_{1}} Z_{2} J_{0}\left(u_{2} I_{2}\right)-J_{1}\left(u_{2} I_{2}\right)}
$$

where

$$
Z_{2}=\frac{Y_{1}\left(u_{1} r_{2}\right)-\frac{Y_{1}\left(u_{1} b\right)}{J_{1}\left(a_{1} b\right)} J_{1}\left(u_{1} I_{2}\right)}{Y_{0}\left(u_{1} r_{2}\right)-\frac{Y_{1}\left(u_{1} b\right)}{J_{1}\left(a_{1} b\right)} J_{0}\left(u_{1} r_{2}\right)}
$$

Equations (D-23) and (D-24) are combined to give the transcendental eigenvalue equation for the $\mathrm{TE}_{0 \mathbf{2}}$ modes,

$$
\frac{\frac{u_{2}}{u_{1}} Z_{2} J_{0}\left(u_{2} r_{2}\right)-J_{1}\left(u_{2} r_{2}\right)}{\frac{u_{2}}{u_{1}} Z_{1} J_{0}\left(u_{2} r_{1}\right)-J_{1}\left(u_{2} r_{1}\right)}=\frac{\frac{u_{2}}{u_{1}} Z_{2} Y_{0}\left(u_{2} r_{2}\right)-Y_{1}\left(u_{2} I_{2}\right)}{\frac{u_{2}}{u_{1}} Z_{1} Y_{0}\left(u_{2} I_{1}\right)-Y_{1}\left(u_{2} r_{1}\right)},
$$


where $Z_{1}$ and $Z_{2}$ are presented in Eqs. (D-24) and (D-26).

\section{Modal Solutions}

Solutions to Egs. (D-15) and (D-27) that lead to guided propagating modes require that $\beta$ be imaginary and thos $\beta^{2}<0$. In the frequency regions where $\beta^{2}>0$, modes experience attenuation only and thus die out rapidly. The cutoff frequency, $\omega_{\mathrm{s}}$, is defined as that frequency above which free wave propagation occurs and below which only modal attenuation occurs. In this mulilayer dielectric waveguide where relative permittivities can be complex, modal solutions for $\beta$ are also complex, having both real and imaginary components, when $\omega>\omega_{\mathrm{c}}$. Field waves are then freely propagating yet attenuated.

As discussed in Appendix A, a TEM mode can exist in a waveguide structure only if $E_{z}$ and $\mathrm{H}_{z}$ both equal zero for all values of $\rho$. Then Eqs. (D-1a) and (D-3a) must be simultaneously zero at $\rho=r_{1}$, as will Eqs. (D-16a) and (D-17a). Similar sets of equations can be identified for the $\rho=I_{2}$ interface. Using the $\mathrm{TM}_{\mathrm{o}_{\mathrm{t}}}$ mode equations, Egs. (D-1a) and (D-3a) at $\rho=r_{1}$, as a baseline to illustrate the situation, the following expressions result:

$$
0=u_{1}^{2}\left[A_{1} J_{0}\left(u_{1} r_{1}\right)+B_{1} Y_{0}\left(\mathrm{u}_{1} r_{1}\right)\right],
$$

and

$$
0=u_{2}^{2}\left[A_{2} J_{0}\left(u_{2} r_{1}\right)+B_{2} Y_{0}\left(u_{2} r_{L}\right)\right] .
$$

Solutions to these equations require that $u_{1}=0$ and $v_{2}=0$. But since $k_{1} \neq k_{2}$, except when the two dielectrics are identical, a contradiction exists for the maltilayer media. Thus, no TEM mode is possible. If the static values of the material relative permittivities are such that $\varepsilon_{1}>\varepsilon_{2}$, then the $T M_{01}$ mode is of lowest order for this coaxial transmission line stnicture. This mode exists at all frequencies and is cutoff only at $\omega=0$. 


\section{REFERENCES}

1. (a) H. Bassen and R. Peterson, "Complete measurement of electromagnetic ftelds with electro-optic crystals," in Blological Effects of Electromagnetic Waves, Selected Papers from USNCURSI Annual Meeting, Vol. II, C.C. Johnson, ed., HEW Publication (FDA)77-8011, pp. 310-323 (1976).

(b) A. J. Rogers, "Optical methods for measurement of voltage and current in power systems," Optics and Laser Tech., 9, pp. 273-283 (1977).

(c) K. Kyuma, S. Tai, M. Nunoshita, N. Mikami and Y. Ida, "Fiber optic cument and voltage sensor using a $\mathrm{Bi}_{12} \mathrm{GeO}_{20}$ single crystal," IEEE J. Lightwave Tech, LT-1, pp. 93-97 (1983).

(d) J. C. Wyss, "Photonic sensors for electromagnetic field measurements," IEEE Ant. and Prop. Newsletter, 26, pp. 5-9 (1984).

2. G. A. Massey, D. C. Erickson and R. A. Kadlec, "Electromagnetic field components: their measurement using electro-optic and magneto-optic effects," Appl. Opt., 14, pp. 2712-2719 (1975).

3. F. Buckholtz, K. P. Koo, A. D. Kersey and A. Dandridge, "Fiber optic magnetic sensor development," Prac. SPIE, 718, pp. 56.65 (1986).

4. Atlantic Research Corp., "High sensitivity RF current probe," specification $\mathbf{\$ 5 2}$ (1969).

5. J. G. Hewith, Jr., "Instrumentation for making broadband measurements on electro-explasive devices," Sixth Symposium on Electroexplosive Devices, Denver Research Institute Rpt. (1969).

6. (a) S. Gibes, E. Jensen, M. Sin and K. Wickersheim, "An improved fiberoptic current sensing system for high frequency susceptibility testing," EMC Technology, 6, No. 7, pp. 45-50 (1987).

(b) R. W. Cernosek, "High frequency fluoroptic thermometry current sensing for 
weapon susceptibility testing," EMC EXPO '88 Symposium Record, Washington, D.C. (1988).

(c) T. H. Grady, "New instrument reduces hazards in EED tests," EMC Technology, 9, No. 3, pp. 17.20 (1990).

(d) $\mathrm{K}$. A. Wickersheim and M. H. Sun, "Small minimally perturbing fiberoptic sensor for measurement of microwave fields and power," Fifth Conf. on High Power Microwave Tech, West Point, N.Y. (1990).

7. (a) A. Yariv and H. Winsor, "ProposaI for detection of magnetic fields through magnetostrictive perturbations of optical fiber," Opt. Lett, 5, pp. 87-89 (1980).

(b) A. D. Kersey, M. Corke, D. A. Jackson and J. D. C. James, "Detection of de and low frequency ac magnetic fields using all single-mode fibre magnetometer," Electron. Lett., 19, pp. $469-471$ (1983).

8. F. Bucholtz, D. M. Dagenais and K. P. Koo, "High frequency fibre-optic magnetometer with $70 \mathrm{fT} / \sqrt{\mathrm{Hz}}$ resolution," Electron. Lets, 25, pp. 1719-1721 (1989).

9. F. Bucholtz, D. M. Dagenais and K. P. Koo, "Mixing and detection of RF signals in fibre-optic magnetostrictive sensor," Electron. Leth, 25 (1989).

10. S. J. Martin and M. A. Butler, "Wideband optical fiber magnetic field sensor," Proc. SPIE, 566, pp. 197-200 (1985).

11. B. T. Neyer, J. Chang and L. E. Roggles, "Calibrated Faraday current and magnetic field sensor," Proc. SPIE, 566, pp. 201-205 (1985).

12. M. N. Deeter, A. H. Rose and G. W. Day, "Faraday-effect magnetic field sensors based on substituted iron garnets, "Proc. SPIE, 1367, pp. 243-248 (1990).

13. M. N. Deeter, A. H. Rose and G. W. Day, "Fast, sensitive magnetic field sensors based on the Faraday effect in YIG," J. Lighwave Technol, 8, pp. 1838-1842 (1990).

14. R. W. Cernosek, "High freguency response of fiber current sensors with 
noncircular and noncentered coils," Proc. 8th Optical Fiber Sensors Conf., pp. 398-401 (1992).

15. (a) G. W. Day, J. D. O. McFadden, L. R. Veeser, G. I. Chandler and R. W. Cernosek, "Optical fiber sensors for the measurement of pulsed electric currents," AGARD Proc No. 383, Guided Optical Structures in the Military Environment, pp. 8-3 thru 8-9 (1985).

(b) G. W. Day, L. R. Veeser, G. I. Chandler and R. W. Cernosek, "Progress in the design of fiber sensors for the measurement of polsed electric currents," Proc. Workshop on Measurements of Electric Quantities in Pulsed Power Systems, Gaithersburg, Md. (1986).

16. (a) A. J. Rogers, "Optical measurement of current and voltage on power systems," JEE J. Elect. Power Applics., 2 , p. 120 (1979).

(b) A. J. Rogers, "Optical-fibre current measurement," Int. J. Optoelectronics, 3, pp. 391-407 (1988).

(c) A. N. Tobin, A. P. Steer, S. J. Tumer, P. R. B. Farie, D. King, R. P. Tatum, J. D. C. Jones and D. A. Jackson, "Application of an optical fibre corrent sensor to electricity supply protection," Proc. SPIE, 1169, pp. 201-209 (1989).

17. (a) A. Papp and H. Harms, "Magnetooptical current transformer. 1: Principles," Appl. Opt., 19, pp. $3729-3734$ (1980).

(b) H. Aulich, W. Beck, N. Doukalias, H. Harms, A. Papp and H. Schneider, "Magnetoopticai current transformer. 2: Components," Appl Opt, 19, pp. 37353740 (1980).

(c) E. Harms and A. Papp, "Magnetooptical current transformer. 3: Measurements," AppL. Opt., 19, pp. 3741-3745 (1980).

I8. L. Veeser, D. Kanja, B. Fremman, P. Knuse and E. Zimmermann, "Measurement of megampere cutrents with optical fibers," Proc. SPIE, 280, pp. 300-304 (1983).

19. G. I. Chandler, P. R. Forman, F. C. Jahoda and K. A. Klare, "Fiber-optic 
heterodyne phase-shift measurement of plasra current," Appl. Opt, 25, pp. 17701774 (1986).

20. (a) H. S. Lassing, A. A. M. Oomens, R. Woltjer, P. C. T. van der Laan and G. G. Wolzak, "Development of a magneto-optic current sensor for high pulsed currents," Rev. Sci. Instrum, 57, pp. 851-854 (1986).

(b) H. S. Lassing, W. J. Mastop, A. F. G. van der Meer and A. A. M. Oomens, "Plasma current measurement by Faraday rotation in a single-mode fiber," Appl. Opt., 26, pp. 2456-2460 (1987).

21. G. W. Day and S. M. Etzel, "Annealing of bend-induced birefringence in fiber current sensors," Tech. Dig., Conf. on Integrated Opt and Opt. Fiber Comm. Venice, pp. $871-874$ (1985).

22. Dingding Tang, A. H. Rose, G. W. Day and Shelley M. Etzel, "Annealing of linear birefringence in single-mode fiber coils: application to optical fiber current sensors," J. Lightwave Technol, 9, pp. 1031-1037 (1991).

23. M. P. Varnham, R. D. Birch and D. N. Payne, "Helical core circularly birefringent fibres," Tech. Dig., IOOC/ECOC '85, Venice, pp. 135-138 (1985).

24. R. I. Laming and D. N. Payne, "Electric current sensors employing spon highly birefringent optical fibers," J. Lightwave Technol, 7, pp. 2084-2094 (1989).

25. (a) K. Shiraishi, S. Sugaya and S. Kawakami, "Fiber Faraday rotator," Appl. Opt., 23, pp. 1103-1106 (1984).

(b) K. Shiraishi, K. Nishino and S. Kawakimi, "Temperature insensitive fiber Faraday rotator," Appl. Opt., 24, pp. 1896-1897 (1985).

26. P. E. Sanders, D. A. Krone and M. S. Maklad, "Faraday rotator single mode fiber," Proc. SPIE, 566, pp. 317-320 (1985).

27. (a) S. B. Poole, D. N. Payne and M. E. Fermann, "Fabrication of low-loss optical fibres containing rare earth ions," Electron. Lett., 21, pp. 737.738 (1985).

(b) J. E. Townsend, S. B. Poole and D. N. Payne, "Solution doping techniques for 
fabrication of rare earth doped optical fibres," Electron. Leth., 23, pp. 329-331 (1987).

28. S. B. Poole and D. N. Payne, "Special optical fibres," Proc. SPIE, 734, pp. 92103 (1987).

29. M. S. Maklad, P. E. Sanders, E. Dowd and A. Kuczma, "Single mode fibers for sensing applications," Proc. SPIE, 718, pp. 97-109 (1986).

30. (a) A. H. Rose, M. N. Deeter and G. W. Day, "Submicroampere per root $\mathrm{Hz}$, high bandwidth current sensor based on the Faraday effect in Ga:YIG," Proc. 8th Optical Fiber Sensor Conf., pp. 394-397 (Monterey, 1992).

(b) A. H. Rase, M. N. Deeter and G. W. Day, "Subrnictoampere per root Hz, high bandwidth current sensor based on the Faraday effect in Ga:YIG," Opt. Lett, 18, pp. 1471.1473 (1993),

31. R. Wolfe, E. M. Gyorgy, R. A. Lieberman, V. J. Fratello, S. J. Licht, M. N. Deeter and G. W. Day, "High frequency magnetic field sensors based on the Faraday effect in garnet thick films," Proc. 8th Optical Fiber Sensors Conf., pp. 390-393 (1992).

32. J. A. Gaj, R. R. Galazka and M. Nawrocki, "Giant exciton Faraday rotation in $\mathrm{Cd}_{1-x} \mathrm{Mn}_{x}$ Te mixed crystals", Solid State Commun., 25, pp. 193-195 (1978).

33. A. D. Kersey, F. Bucholtz and A. Dandridge, "Sensitivity-bandwidth limitation in optical-Fiber Faraday-rotation current sensors," Intl. J. Optoelectronics, 3, pp. $323-332$ (1988).

34. R. W. Cemosek, "Expanded coaxial transmission cells for electromagnetic testing," Sandia National Laboratories Technical Rpt, SAND91-0768 (1991).

35. H. Piller, "Faraday Rotation," Ch. 3 in Semiconductors and Semimetals, Vol. 8, R. K. Willardson and A. C. Beer, eds. (Academic Press, 1972).

36. P. A. Williams, A. H. Rose, G. W. Day, T. E. Milner and M. N. Deeter, "Temperature dependence of the Verdet constant in several diamagnetic glasses," 
Appl. Opt, 30, pp. 1176-1178 (1991).

37. M. A. Butler and E. L. Venturini, "High frequency Faraday rotation in FR-5 glass," Appl. Opt, 26, pp. 1581-1582 (1987).

38. M. A. Butler, S. J. Martin and R. J. Baughmen, "Frequency-dependent Faraday rotation in CdMnTe," Appl. Phys. Leth, 49, pp. 1053-1055 (1986).

39. M. J. Weber, "Faraday rotator materials for laser systems," Proc. SPIE, 681, pp. $75-90(1986)$.

40. (a) M. J. Weber, "Faraday rotator materials," Lawrence Livermore Laboratory Rpt., M-103 (1982).

(b) M. J. Weber, "Magnetooptical materials," Sect. 2-2 in Handbook of Laser Science and Technology, Vol. II, Optical Materials, Pant 2, M. J. Weber, ed. (CRC Press, 1986).

41. S. C. Rashleigh and R. Ulrich, "Magneto-optic current sensing with birefringent fibers," Appl. Phys. Lett, 34, pp. 768-770 (1979).

42. W. F. Hernsing, "VISAR: 2.5 minutes for data reduction," Proc. SPIE, 427, pp. 199-202 (1983).

43. H. J. Arditty, Y. Bourbin, M. Mapuchon and C. Puech, "Current sensor using state-of-the-art fiber optic interferometric techniques," Tech. Dig. Third Intl. Conf. on Integrated Opt. and Opt. Fiber Comun, San Francisco (1981).

44. (a) L. R. Veeser and G. W. Day, "Faraday effect current sensing using a Sagnac interferometer with a $3 \times 3$ coupler," Proc. 7th Optical Fibre Sensors Conf., pp. 325-328 (1990).

(b) K. B. Rochford, G. W. Day and P. R. Forman, "Polarization dependence of response functions in $3 \times 3$ Sagnac optical fiber current sensors," Proc. 9th Optical Fiber Sensors Conf., Post-deadline 6, Florence, Italy (1993).

45. G. W. Day and A. H. Rose, "Faraday effect sensors: state of the art," Proc. SPIE, 985, pp. 138-150 (1988). 
46. G. W. Day, K. S. Lee, A. H. Rose, L. R. Veeser, B. J. Papatheofanis and H. K. Whitesel, "Optical fiber sensors for electromagnetic quantities," Proc. 34th Intl. Instrumentation Symp., ISA, Albuquerque, N.M., pp. $205-207$ (1988).

47. A. M. Smith, "Polarization and magnetooptical properties of single mode optical fiber," Appl Opt, 17, pp. 52-56 (1978).

48. S. C. Rashleigh, "Origins and control of polarization effects in single mode fibers," IEEE J. Lightwave Tech., LT-1, pp. 312-331 (1983).

49. D. N. Payme, A. J. Barlow and J. J. Ramskov Hansen, "Development of low- and high-birefringence optical fibres," IEEE J. Quantum Electron., QE-18, pp. 477 488 (1982).

50. R. Ulrich, S. C. Rashleigh and W. Eickhoff, "Bending induced birefringence in single mode fibers," Opt. Lett., 5, pp. 273-275 (1980).

51. (a) R. C. Jones, "A new calculus for the treatment of optical systems," J. Opt. Soc. $A m, 31$, p. 488 (1941).

(b) W. J. Tabor and F. S. Chen, "Electromagnetic propagation through materials possessing both Faraday rotation and birefringence: experiments with ytterbium orthoferrite, "J. Appl. Phys., 40, p. 2760 (1969).

52. R. Ulrich and A. Simon, "Polarization optics of twisted single mode fibers," Appl. Opt., 18, pp. 2241-2251 (1979).

53. L. Li, J. R. Qian and D. N. Payne, "Current sensing using highly birefringent bow-tie fibers," Electron. Lett, 22, pp. 1142-1144 (1986).

54. Dingding Tang and G. W. Day, "Progress in the development of miniatare optical fiber cument sensors," Tech. Dig., IEEE Lasers and Electro-Optic Soc. Annual Conf., Santa Clara, Ca. (1988).

55. P. A. Williams, G. W. Day and A. H. Rose, "Compensetion for the temperature dependence of the Faraday effect in diamagnetic materials: applications to optical fibre sensors," Electron. Lett, 27, pp. 1131-1132 (1991). 
56. M. A. Butler, "Wavelength and temperature dependence of the Faraday effect in $\mathrm{Cd}_{\mathrm{-x}} \mathrm{Mn}_{\mathrm{x}} \mathrm{Te}$," Solid State Commun., 62, pp. $45-47$ (1987).

57. N. Kullendorff and B. Hok, "Temperature independent Faraday rotation near the band gap in $\mathrm{Cd}_{1-\mathrm{x}} \mathrm{Mn}_{\mathrm{x}} \mathrm{Te}{ }^{n}$ Appl. Phys. Lett., 46, pp. 1016-1018 (1985).

58. O. Kamada, H. Minemoto and S. Ishizuka, "Mixed rare earth gamet (TbY)IG for magnetic freld sensors," J. Appl Phys., 61, pp. 3268-3270 (1987).

59. G. W. Day. "Compact sensors for the measurement of low level electric currents," Tech. Dig., Ath Inll. Conf. on Opt Fiber Sensors, Tokyo (1986).

60. R. W. Cernosek, "Travelling wave Faraday effect fiber current sensors," Proc. 7th Optical Fibre Sensors Conf., pp $72-76$ (1990).

61. A. Sommerfeld, Electrodynamics, pp. 177-193 (Acádemic Press, 1952).

62. A. Yariv, Optical Electronics, 3rd. ed., Ch. 9 (Holt, Rinehart and Winston, 1985).

63. G. W. Day, "Recent advances in Faraday effect sensors," Optical Fiber Sensors, Proceedings of the 6th Intemational Conference, OFS '89, H. J. Arditty, J. P. Dakin and R. T. Kersten, eds., pp. 250-254 (Springer-Verlag, Berlin, 1989).

64. C. T. Chang, J. A. and H. F. Taylor, "Fibre-optic delay-line devices for RF signal processing," Electron. Lett., 13, p. 678-680 (1977).

65. K. P. Jackson, S. A. Newton, B. Moslehi, M. Tur, C. C. Cutler, J. W. Goodman and H. J. Shaw, "Optical fiber delay-line signal processing," JEEE Trans. Microwave Theory Tech, MTT-33, pp. 193-209 (1985).

66. C. M. Gee, G. D. Tharmond and H. W. Yen, "17 GHz bandwidth electro-optic modulator," Appl. Phys. Lett., 43, pp. 998-1000 (1983).

67. R. C. Weast, ed., Handbook of Physics and Chemistry, 54th ed., sect. E (CRC Press, 1973).

68. K. A. Wickersheim and R. V. Alves, "Recent advances in optical temperature measurement," Industrial Res. and Dev., 21, p. 82 (1979).

69. C. G. Malmberg and A. A. Maryott, "Dielectric constant of water from $0^{\circ}$ to 
$100^{\circ} \mathrm{C}, "$ J. Res. Nat'l Bur. Stand., 56, pp. 1-8 (1950).

70. B. B. Owen, R. C. Miller, C. E. Milner and H. L. Cogan, "The dielectric constant of water as a function of temperature and pressure," J. Phys. Chem, 65, pp. 20652070 (1961).

71. C. Kittel, Introduction to Soltd State Physics, 2nd. ed., pp. 175-179 (Wiley, 1956).

72. J. B. Hasted, "The experimental data for liquid water," Ch. 2 in Aqueous Dielectrics, (Chapman and Halt, London, 1973).

73. J. R. Wait, Electronagnetic Wave Theory (Harper and Row, New York, 1985).

74. D. E. Amos, "A subroutine package for Bessel functions of a complex argument and nonnegative order," Sandia National Laboratories Technical Rpt, SAND851018 (1985).

75. R. W. Cernosek, "High frequency models for travelling wave optical fiber current sensors," Tech. Dig., 1992 Optical Society of America Annual Meeting, Albuquerque, N.M., p. 20 (1992).

76. R. E. Bolz and G. L. Tuve, eds., Hondbook of Tables for Applied Engineering Sclence, 2nd ed., Sect. 2 (CRC Press, 1979).

77. W. W. Macalpine and R. O. Schildknecht, "Coaxial resonator with helical inner conductor," Proc. IRE, pp. 2099-2105 (1959).

78. M. L. Crawford, "Generation of standard EM fields using TEM transmission cells," IEEE Trans. Electromagn. Compat, EMC-16, pp. 189-195 (1974).

79. M. L. Crawford and J. L. Workman, "Using a TEM cell for EMR measurements of electronic equipment," NBS Technical Note 1013 (1981).

80. T. Moreno, Microwave Transmission Design Data (Artech House, 1989).

81. C. E. Baum, "The conical transmission line as a wave launcher and terminator for a cylindrical transmission line, "Air Force Weapons Laboratory Sensor and Sinulation Nores, Vol. 2, EMP 1-2, Note SSN 31 (1967). 


\section{GLOSSARY OF SPECIAL SYMBOLS AND TERMS}

c

d

$d / x_{0}$ $\mathrm{dW} / \mathrm{dV}_{0}$ $\mathrm{dW} / \mathrm{dz}$

n

n

r

$\mathbf{r}_{1,2}$

$\mathbf{r}_{\mathfrak{f}}$

t

L.b

$4,1,2, \ldots$

v

$\mathrm{u}_{1,2}$

पm

$\mathrm{v}$

$v_{\mathbf{f}}$

radius of coaxial transmission line inner (center) confuctor

radial distance from coaxial line axis to nearest point on fiber segment

radius of coaxial line outer conductor

speed of light in vacuum

diameter of fiber coil

ratio of diameter to pitch of helical fiber coil

energy density for coaxial line dielectric

total energy per unit length along axis in a coaxtal line dielectric

magnitude of the electric charge

modulation frequency

cutoff frequency of higher order modes in coaxial transmission lines

cutoff frequency (first null) in optical fiber sensor response

resonance center frequency

fiber twisı material parameter

half-height of rectang

static or steady state current amplitude in a conductor

average optjcal detector output current

$\sqrt{-1}$

electromagnetic wave vector

wave propagation parameter (general)

wave parameter for dielectric regjons 1,2

Boltumann's constant

fiber length variable (general)

length vector

short fiber or optical material Iength (usually less than one turn)

length of fiber lead

integer counter for serjes, azimuthal mode number in coaxial transmission line

integer counter for response resonances

fiber refractive index

surface norma] vector

radial distance from conductor axis to tiber axis

inner, outer radius of fiber mandrel, radii at dielectric transitions

fjber radius

time variable (general)

time at points $a, b$ on a short circular fiber loop

time or time delay at designated points: starting or reference, $1,2, \ldots$ in a fiber

coil or lead

Bessel function wave number, $u^{2}=k^{2}+\beta^{2}$

Bessel function wave number for dielectric regions 1,2

Bessel function wave number for the mnth coaxial waveguide mode

velocity variable (general)

velocity of light in fiber 


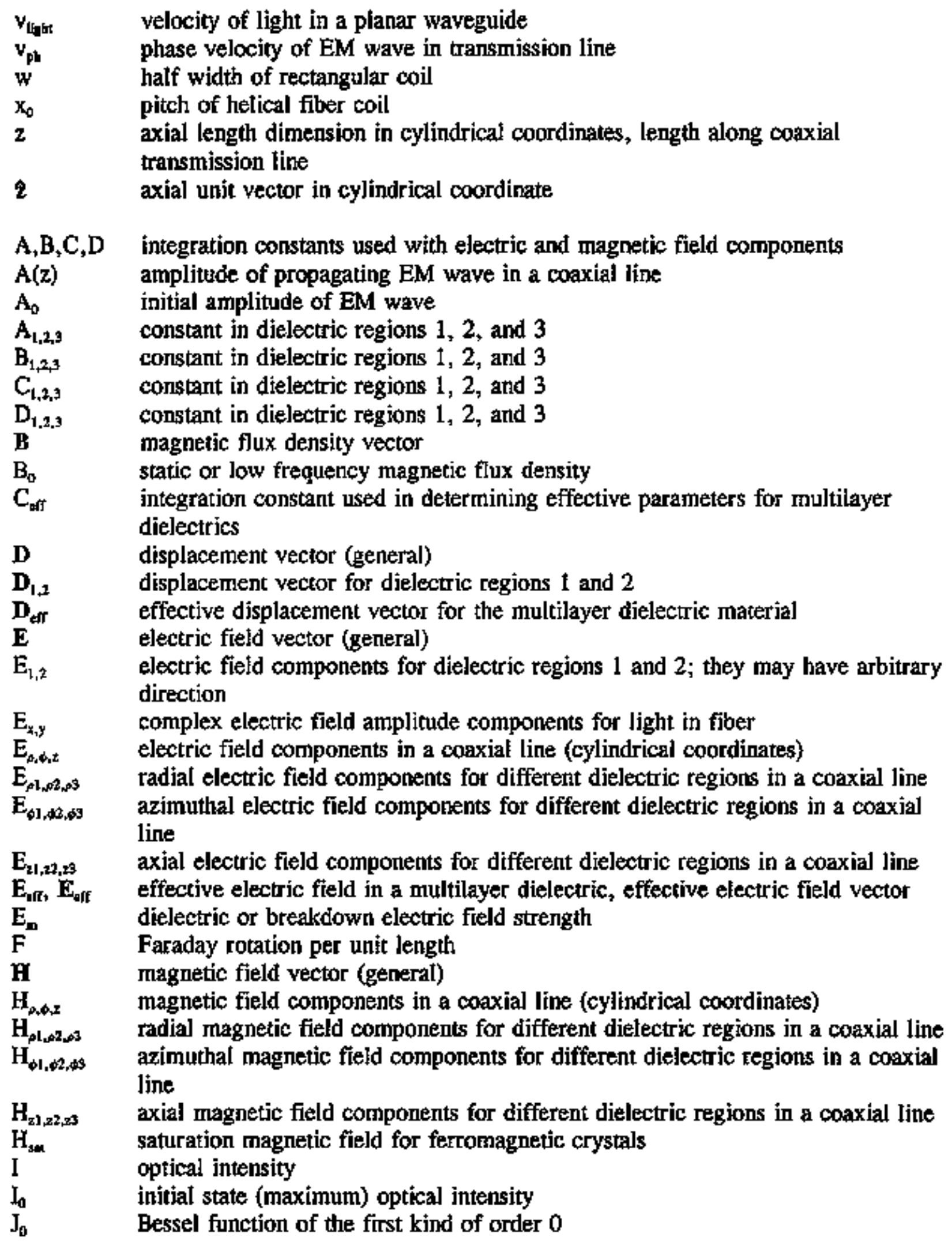




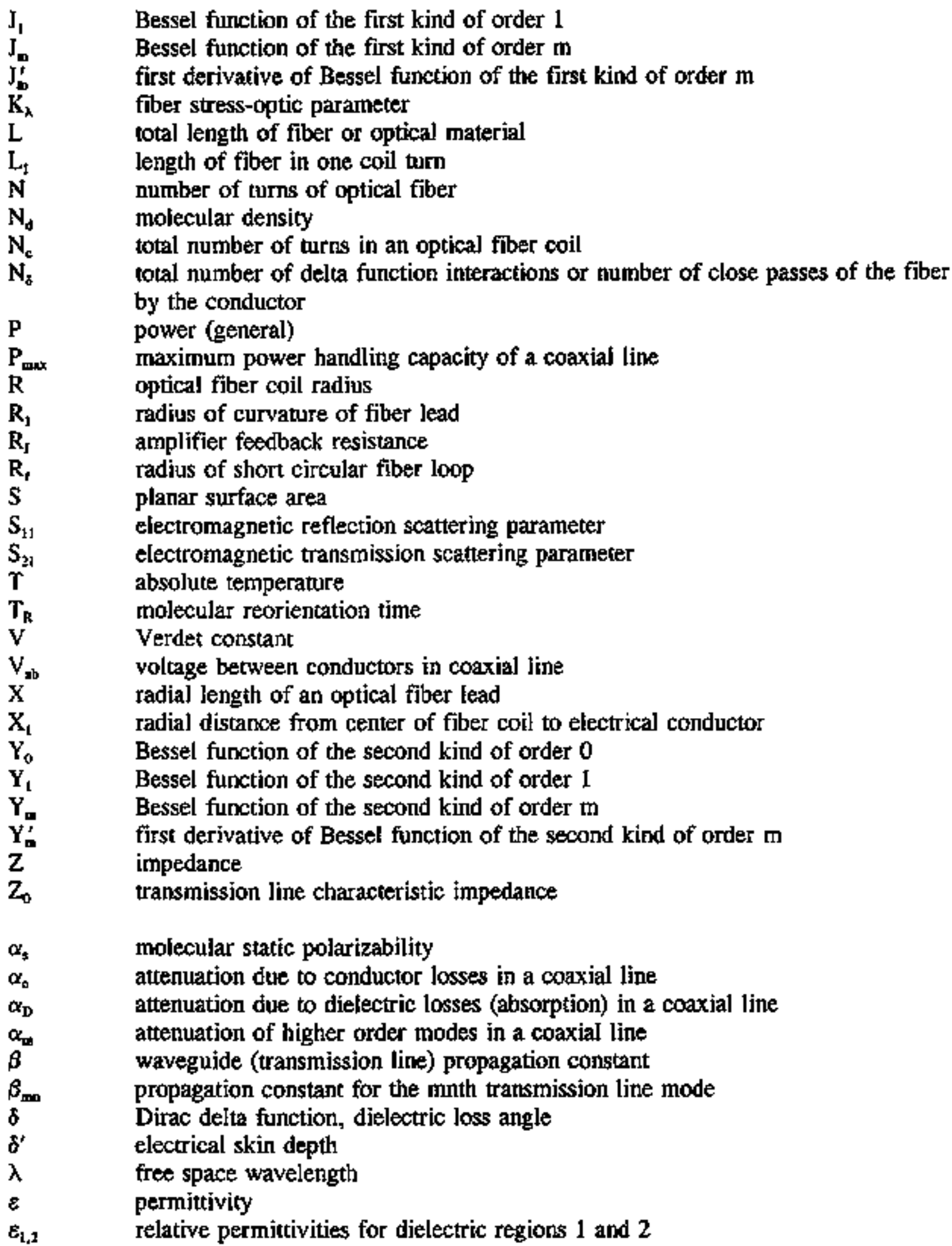




\begin{tabular}{|c|c|}
\hline $\begin{array}{l}E^{\prime}, \varepsilon^{\prime \prime} \\
\varepsilon_{0}\end{array}$ & $\begin{array}{l}\text { real and imaginary components of relative permittivity } \\
\text { free-space permittivity }\end{array}$ \\
\hline$\varepsilon_{\mathrm{I}}$ & material retative permittivity (complex quantity) \\
\hline$\varepsilon_{s}$ & static (low frequency) relative permittivity \\
\hline$\varepsilon_{\text {eff }}$ & effective relative permittivity \\
\hline $\begin{array}{l}\mu \\
\mu_{0}\end{array}$ & permeability \\
\hline $\begin{array}{l}\mu_{0} \\
\mu_{\mathrm{T}}\end{array}$ & free-space permeability \\
\hline $\begin{array}{l}\mu_{\mathbf{r}} \\
\mu_{\mathrm{r}}\end{array}$ & material relative permeability \\
\hline $\begin{array}{l}\mu_{t} \\
\eta\end{array}$ & fiber material permeability \\
\hline $\begin{array}{l}\eta \\
k\end{array}$ & electromagnetic wave impedance \\
\hline $\begin{array}{l}k \\
\phi\end{array}$ & permittivity constant, $\varepsilon_{\mathrm{s}}-1$ \\
\hline$\hat{\phi}$ & $\begin{array}{l}\text { aximuthal angle in cylindrical coordinates } \\
\text { azimuthal unit vector }\end{array}$ \\
\hline$\phi^{\prime}$ & total birefringence in optical medium \\
\hline$\varsigma^{\prime}$ & phase matching parameter in planar waveguide sensor \\
\hline$\rho$ & radial dimension in cylindrical coordinates \\
\hline$\hat{\boldsymbol{p}}$ & radial dimension unit vector \\
\hline o & conductivity \\
\hline$\theta_{1,2}$ & $\begin{array}{l}\text { taper angles for the center and outer conductors in a conical transmission line } \\
\text { launcher }\end{array}$ \\
\hline$\xi$ & $\begin{array}{l}\text { ratio of time delay to fight transit time in a single fiber turn (fraction indicating } \\
\text { light entry point in the fiber coil) }\end{array}$ \\
\hline$\xi_{r}$ & number of twists per unit tength in optical fiber \\
\hline$\omega$ & angular modulation frequency (general) \\
\hline$\omega_{\mathrm{c}}$ & cutoff angular frequency of higher order modes in coaxial transmission lines \\
\hline$\omega_{\mathrm{co}}$ & cutoff angular frequency (first null) in optical fiber sensor response \\
\hline $\begin{array}{c}\omega_{k} \\
\omega_{\mathrm{k}}\end{array}$ & $\begin{array}{l}\text { angular resonant frequency } \\
\text { angular freguency at which rotation relative magnitude is reduced } 3 \mathrm{~dB}\end{array}$ \\
\hline$\omega \tau / 2$ & $\begin{array}{l}\text { angular frequency at which rotation relative magnitude is reduced } 3 \mathrm{~dB} \\
\text { normalized frequency for optical fiber coil sensor }\end{array}$ \\
\hline$\omega \tau_{\mathrm{j}} / 2$ & normalized frequency for a single-turn fiber sensor \\
\hline $\begin{array}{l}\tau_{1} \\
\tau_{1}\end{array}$ & transit time of light through fiber \\
\hline $\begin{array}{l}\tau_{1} \\
\tau_{d}\end{array}$ & transit time of light through one turn of fiber \\
\hline $\begin{array}{c}T_{d} \\
T_{z}\end{array}$ & light propagation delay time between fiber coils \\
\hline$\tau_{\mathrm{g}}$ & transit time of light through length of fiber $\ell$ \\
\hline$\tau_{\mathrm{R}}$ & $\begin{array}{l}\text { material magnetic ion telaxation time } \\
\text { normalized frequency parameter for traveling wave sensor }\end{array}$ \\
\hline$\infty$ & intinity \\
\hline$\alpha$ & rotary angle between dl and an arbitrary reference point on a fiber loop \\
\hline$\alpha^{\prime}, \beta^{\prime}$ & angles in the fiber coil plane used to locate a light segment in a fiber lead \\
\hline $\begin{array}{l}\alpha_{0} \\
\beta_{\max }^{\prime}\end{array}$ & $\begin{array}{l}\text { initial value of } \alpha \text { at time } \mathrm{t}=0 \\
\text { maximum yalue of the angle } \beta^{\prime}\end{array}$ \\
\hline$\gamma$ & angle between the magnetic flux density vector $B$ and the fiber length vector dl \\
\hline & \\
\hline
\end{tabular}


$\psi \quad$ angle between the wave vector $\mathbf{k}$ and the fiber length vector di out of the plane of the fiber coil

$\triangle B \quad 3 \mathrm{~dB}$ bandwidth

$\Delta \mathrm{L} \quad$ change in total fiber length in helical coil

$\Delta \dot{P} \quad$ linear birefringence or retardance per unit length

$\mathbf{Q}^{\prime} \quad$ twisting rotation per unit length

$\theta$ rotation in the plane of polarization (general); ustally refers to Faraday rotation

$\theta(\omega) \quad$ frequency-dependent rotation

$\theta_{6}(\omega)$ frequency dependent rotations due to delta function interactions

$\theta_{0} \quad$ static or low frequency rotation

$\theta_{1,2,3, \ldots}(\omega)$ totations in different segments of fiber

$\Theta_{\mathrm{m}}(\omega)$ frequency and material dependent Faraday rotation in paramagnetic crystals and glasses

$\theta_{\gamma}(\omega)$ frequency dependent rotation in short fiber length $\ell$

$\Theta_{\mathrm{F}}^{\text {gat }} \quad$ saturation rotation in ferromagnetic material

ANA automatic network analyzer

APD avalanche phorodiode

BEF bandwidth enhancement factor

DC (or dc) direct current

EM electromagnetic

EMI electromagnetic interference

HOM higher order modes

MM multimode (fiber)

NEI noise equivalent current

PIN positive-intrinsic-negative

$\mathrm{RF} \quad$ radio frequency

SBP sensitivity-bandwidth product

SM singlemode (fiber)

S/N signaI-to-noise ratio

SWR standing wave ratio

TE transverse electric

$T E_{m a}$ mnth transverse electric mode

TEM transverse electromagnetic

TM transverse magnetic

$T M_{\text {po }}$ mnth transverse magnetic mode

UV ultraviolet

YIG yttrium iron garnet

Ga:YIG gallium substituted YIG 


\section{DISTRIBUTION:}

1 Gordon W. Day

National Institute of Standards and Technology

Mail Code 815.00

325 Broadway

Boulder, CO 80303

1 Allen H. Rose

National Institute of Standards and Technology

Mail Code 815.03

325 Broadway

Boulder, CO 80303

5 MS $0351 \quad$ R.W. Cernosek, Org. 1315

1 MS 0351 J.J. Wiczer, Org. 1315

1 MS 0175 W.W. Plummer, Org. 13214

1 MS 0899 Central Technical Files, Org. 8523-2

5 MS 0899 Technical Library, Org. 13414

1 MS 0619 Technical Publications, Org. 13416

10 MS 0100 Document Processing for DOE/OSTI, Org. 7613-2 